

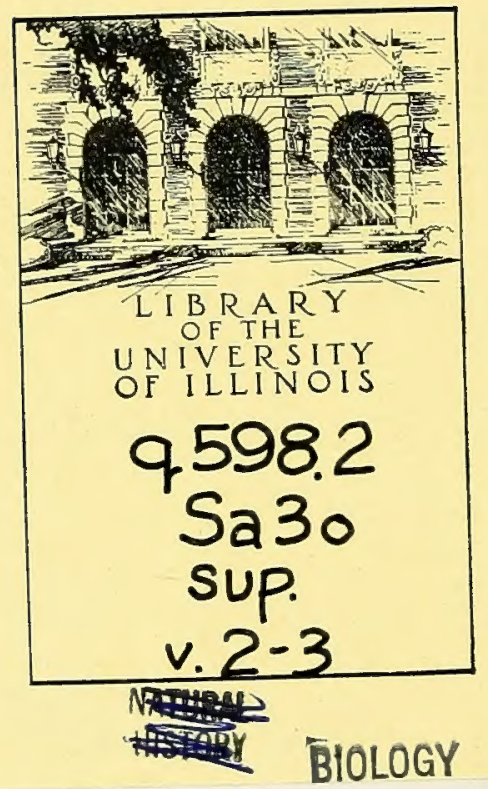


Digitized by the Internet Archive in 2011 with funding from University of Illinois Urbana-Champaign 





\section{AGGIUNTE}

ALLA

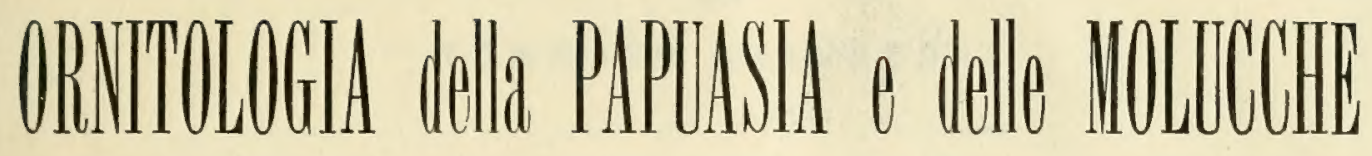

PER

TOMMASO SALVADORI

PARTE SEGONDA

PASSERES

T O R IN 0

C A R L O CLA US E N

Libraio della R. Accademia delle Scienze

1890 
Estr. dalle Memorie della Reale Accademia delle Scienze di Torino, Serie II, Tom. XL.

Torino, Stamperia Reale-Paravia. 3187 (100) 26-III 90 


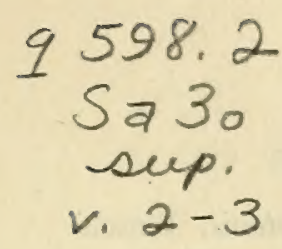

\section{Nat. Hiat.}

\section{AGGIUNTE}

AI.LA

\section{Ornitologia della Papuasia e delle Molucche \\ PER}

TOMMASO SALVADORI

\section{PARTE SECONDA \\ PASSERES}

Appr. nell'adunanza del 17 Novembre 1889

\section{PREFAZIONE}

In questa seconda parte delle Aggiunte alla Ornilologia della Papuasia $e$ delle Molucche vengono passate in rassegna le specie dell'Ordine dei Passeres. Alle specie di quest'Ordine, annoverate nel vol. II della Ornito. logia, sono da aggiungerne 89 , delle quali do qui l'elenco:

1. Monarcha erythrostictus (Sharpe).

2. 》 rufocastaneus, Ramsay.

3. " buruensis, Meyer.

4. $\gg$ castus, Sclat.

5. $\gg$ browni, Ramsay.

6. Heteranax mundus (Sclat.).

7. Arses henkei, Meyer.

8. Rhipidura lenzi, Blas. (?)

9. \ fusco-rufa, Sclat.

10. $»$ hamadryas, Sclat.

11. " opisterythra, Sclat.

12. 》 fallax, Ramsay.

13. Myiagra fulviventris, Sclat.

14. Poecilodryas minor, Meyer (?)

15. Erythromyias (?) riedeli, Büttik.

16. Microeca hemixantha, Sclat.

17. Gerygone dorsalis, Sclat.

18. $\gg$ fulvescens, Meyer.

19. 》 cinereiceps (Sharpe).

20. \# bimaculata, Meyer.
21. Graucalus unimodus, Sclat.

22. " timorlaoensis, Meyer.

23. 》 holopolius, Sharpe.

24. Edoliisoma nehrkorni, Salvad. nov.sp.

25. " erythropygium, Sharpe.

26. Lalage moesta, Sclat.

27. Artamus musschenbroccki, Meyer.

28. Dicruropsis guillemardi, Salvad, n. s.

29. \ propinqua (Tristr.).

30. Cracticus louisiadensis, Tristr.

31. Rhectes meridionalis, Sharpe (?)

32. 》 rubiensis, Meyer.

33. Pachycephala fusco-flava, Sclat.

34. » sharpei, Meyer.

35. \# meyeri, Salvad.nov.sp.

36. \$ arctitorquis, Sclat.

37. " liebirensis, Meyer.

38. Pachycephalopsis fortis (Gadow).

39. Sittella albifrons, Ramsay.

40. Hermotimia cristianae, Tristr. 
41. Dicaeum nitidum, Tristr.

42. 》 pulchrius, Sharpe.

43. 》 salvadorii, Meyer.

44. " fulgidum, Sclat.

45. 》 tristrami, Sharpe.

46. Myzomela ramsayi, Finsch.

47. 》 rubro-cucullata, Tristr.

48. 》 wakoloensis, Forbes.

49. 》 Kleinschmidti, Sharpe.

50. 》 annabellae, Sclat.

51. 》 (?) sharpei, Grant (?)

52. Melipotes fumigatus, Meyer.

53. Melidectes emilii, Meyer.

54. Melirrhophetes batesi, Sharpe (?)

55. Stigmatops salvadorii, Meyer.

56. 》 kebirensis, Meyer.

57. Ptilotis fulvo-cinerea, Meyer.

58. Philemon timorlaoensis, Meyer.

59. Zosterops crissalis, Sharpe.

$60 . \quad \gg$ griseiventris, Sclat.

61. Pitta finschi, Ramsay.

62. Turdulus machiki (Forbes).

63. " schistaceus (Meyer).

64. Corydalla richardi (Vieill.).

(?). Mirafra javanica, Horsf.
65. Donacicola hunsteini, Finsch.

66. Erythrura tricolor (Vieill.).

67. Calornis circumscripta, Meyer.

68. \% crassa, Sclat.

69. Mimeta decipiens, Sclat.

70. Corvus latirostris, Meyer (?)

71. Macrocorax woodfordi, Grant.

72. Manucodia rubiensis, Meyer.

73. Phonygama purpureo-violacea, Meyer.

74. 》 thomsoni, Tristr.

75. Parotia lawesi, Ramsay.

76. Lophorhina minor, Ramsay.

77. Astrarchia stephaniae, Finsch.

78. Epimachus macleayae, Ramsay.

79. 》 meyeri, Finsch.

80. Drepanornis cervinicauda; Sclat.

81. Paradisea finschi, Meyer.

82. 》 guilielmi II, Cab.

83. " augustae victoriae, Cab.

84. 》 decora, Salv. et Godm.

85. Diphyllodes jobiensis, Meyer.

86. 》 hunsteini, Meyer.

87. Paradisornis rudolphi, Finsch.

88. Amblyornis subalaris, Sharpe.

89. Aeluroedus melanocephalus, Ramsay.

Di queste 89 specie, una, la Corydalla richardi (Vieill.), fu da me ommessa per inavvertenza nella Ornitologia, un'altra, la Erythrura tricolor (Vieill.), è stata trovata recentemente nelle isole del gruppo di Timor-laut, 84 sono state denominate e descritte negli ultimi tempi e tre finalmente, la Edoliisoma nehrkorni, la Dicruropsis guillemardi e la $\mathrm{Pa}$ chycephala meyeri, vengono denominate e descritte ora da me. Parecchie specie, segnate con punto interrogativo, mi sembrano non sufficientemente stabilite.

Io debbo vivissime grazie al Dr. A. B. Meyer, Direttore del Museo di Dresda, il quale $\mathrm{mi}$ ha inviato molti esemplari tipici, preziosissimi, affinchè potessi farne uno studio comparativo.

Torino, Museo Zoologico, 17 Novembre 1889. 


\section{ORDO PASSERES}

\section{(256) Hirundo gutturalis, Scop.}

Hirundo gutturalis, Meyer, Sitzb. u. Abh. Gesellsch. Isis, 1884, p. 21 (Ceram, Riedel). - Pleske, Bull. Acad. Petersb. XXIX, p. 528 (Ternate) (1884). - Sharpe, Cat. B. X, p. 134 (1885). - Guillem., P. Z. S. 1885, p. 569 (Batchian).

\section{(257) Hirundo javanica, SparrM.}

Hirundo javanica, Salvad., Voy. Chall., Birds, p. 78 (1881). - Sharpe, Journ. Linn. Soc., Zool. XVI, p. 430 (Morocco) (1882). - Meyer, Sitzb. u. Abh. Gesellsch. Isis, 1884, Abh. I, p. 22 (Sangi, Meyer, Timorlaut, Riedel). - Forbes, P. Z. S. 1884, pp. 426, 433. - Pleske, Bull. Acad. Petersb. XXIX, p. 528 (Ternate) (1884). - Nehrk. Journ. f. Orn. 1885, p. 32 (Waigeu). - Sharpe, Cat. B. X, p. 142 (1885). - Ramsay, Tab. List, p. 2 (1888).

(258) Hirundo tahitica, GM.

Hirundo tahitica, Finsch, Ibis, 1881, p. 536 (New Britain). - Ramsay, Pr. Linn. Soc. N. S. W. VII, 1. 22 (1882). - Tristr., Ibis, 1882, p. 142. - Layard, Ibis, 1882, pp. 502, 543. - Finsch, Vög. d. Südsee, p. 5 (Nova Britannia) (1884). - Sharpe, Cat. B. X, p. 141 (1885). - Grant, P. Z. S. 1887 , p. 330 (Alu); 1888, p. 194 (Guadalcanar).

Hab. in Nova Britannia (Finsch, Kleinschmidt); Nova Caledonia (Layard).

\section{Gen. PETRochelidon, Cab.}

Petrochelidon, Cab., Mus. Hein. I, p. $47(1850)$. . . . . . . . Himundo melanogaster, Sw, Hylochelidon, Gould., Handb. B. Austr. I, p. 111 (1865) . . . . . . Hirundo nigricans, Vieill.

\section{(259) Petrochelidon nigricans (VIEILL.).}

Hylochelidon nigricans, Salvad., Voy. Chall., Birds, p. 78 (1881). - Meyer, Zeitsch. f. ges. Orn. I, p. 279 (1884).

Hirundo nigricans, Finsch, Ibis, 1881, p. 536 (New Britain). - Id., Vög. d. Südsee, p. 5 (Nova Britannia) (1884).

Petrochelidon nigricans, Sharpe, Report Alert, p. 21 (1884). - Id., Cat. B. X, p. 190 (1885)。 Id., Mon. Hirund. pt. V, VI, pl. 2 (1887). - Ramsay, Tab. List, p. 3 (1888). - Buller, B. of New Zeal. I, p. 74 (1888).

Le isole di Timor e Flores debbono essere tolte dall'habitat di questa specie, giacchè gli esemplari delle medesime sono considerati dallo Sharpe (Cat. B. X, p. 192) come specificamente distinti ( $P$. timoriensis, Sharpe).

\section{(260) Peltops blainvillei (Less. et GarN.).}

Peltops blainvillei, Sharpe, Journ. Linn. Soc., Zool. XVI, p. 430 (Ghoqueri) (1882). - Nehrk., Journ. f. Orn. 1885, p. 32 (Waigeu). - Guillem, P. Z. S. 1885, p. 630 (Waigiou, Mysol, Andai). 
(991) Monarcha richardsil (Ransay).

Piezorhynchus richardsii, Ramsay, Pr. Linn. Soc. N. S. W. VII, p. 24 (1882) (Is. Salomone). Sharpe, Gould's, B. New Guin. pt. XVII, pl. 7 (1884).

Monarcha richardsii, Sclat., P. Z. S. 1883, p. 347 (Rubiana, Is. Szlomone).

(261) Monarcha castaneiventris, VerR.

Pomarea? castaneiventris, Salvad., Orn. Pap. e Mol. II, p. 11 (partim) (1881). - Hamsay, Pr. Linn. Sur. X. S. W. VII, p. 24 (partim) (1883) (Isole Salomone), - Tristro, Ibis, 1882, pp. 135, 142. - Sharpe, Gould's B. New Guin. pt. XVIII, pl. 8 (1884).

Monarcha castaneiventris, Salvad., op. cit. III, p. 529 (1882). - Grant, P. Z. S. 1887, p. 331 (partim); 1888, pp. 194, 195.

Hab. in Papuasia - Ins. Salomonis, Guadalcanar (Cockerel7).

Sulla fede del Ramsay, io riferii (Ibis, 1880, p. 129) a questa specie la sua Moncrcha rufo-castanea, facendo tuttavia notare come la descrizione di questa presentasse notevoli differenze e tali da far dubitare che essa potesse costituire una specie diversa, la quale cosa è ora dimostrata.

\section{Sp. 1064 (261 bis) Monarcha erythrostictus (SHARPE).}

Monarcha castaneiventris, part., Grant, P. Z. S. 1887, p. 331 (specimen b).

Pomarea erythrosticta, Sharpe, P. Z. S. 1888, p. 185.

Monarcha erythrosticta, Grant, P. Z. S. 1888, p. 195 (Fauro).

Foem. ad. Corpore supra, capite et collo nigris, purpureo nitentibus; macula anteoculari lacte cinnamomea; pectore, abdomine, lateribus et subcaudalibus saturate castaneis; tibiis castaneis, basi nigra; subalaribus et axillaribus castaneis, illis in basi nigris; margine alarum nigro; tectricibus inferioribus remigum primariorum cinereis; remigibus subtus fuscis; rostro griseo; pedibus nigris; iride brunnea.

Long. tot. $0^{\mathrm{m}}, 142$; al: $0^{\mathrm{m}}, 080$; caud. $0^{\mathrm{m}}, 056$; rostri culm. $0^{\mathrm{m}}, 017$; tarsi $0^{\mathrm{m}}, 019$.

Hab. in Papuasia - Ins. Salomonis, Fauro (Woodford).

Obs. Monarcha M. castaneiventri, Verr. similis, sed macula antcoculari cinnamomea facite distinguendus.

\section{Sp. 1065 (261 ter) Monarcha rufo-castaneus, RAMSAY.}

Monarcha rufo-castanea, Ramsay, Pr. Linn. Soc. N. S. W. IV, pp. 79, 313 (1879). - Id., Nature, XX, p. 125 (1879). - Salvad., Ibis, 1880, p. 129. - Grant, P. Z. S. 1988, p. 195.

Monarcha rufo-castaneus, Salvad., Ann. Mus. Civ. Gen. XIV, p. 508, n. 108 (1879).

Pomarea? castaneiventris, partim, Ramsay, Pr. Linn. Soc. N. S. W. VII, p. 24 (1882) (Isole Salomone). - Salvad., Urn. Pap. e Mol. II, p. 11 (1881).

Pomarea rufo-castanea, Sharpe, Gould's B. New Guin. pt. XVIII, pl. 7 (1884).

Supra nigro-sctistaceus, purpureo nitens, cervice et interscapulio paullum ci-. nerco tinctis, tectricibus majoribus et remigum primariorum, remigibusque fusconigricuntibus; cauda nigro-caerulescente; lateribus colli et gula obscure cinereis; corpore subtus, tibiis, subcaudalibus et axillaribus castaneis; subalaribus castaneis, sed prope marginem alarum cinereis, castaneo marginatis.

Foem. Supra griseo-schistacea, vertice vix pallidiore; tectricibus majoribus, alula spuria, tectricibus remigum primariarum remigibusque fuscis, subtititer rufescente marginatis; rauda sordide fusco-nigricante; lateribus capitis pallide griscis; 
lateribus colli griscis, rufescente tinctis; gula grisco-schistacea, inferius et collo antico rufescentibus; corpore subtus rufo-castanco; tibiis griseis, rufo tinctis; axillaribus rufo-castaneis; subalaribus griseis, rufo marginatis.

Long. tot. $0^{\mathrm{m}}, 139-0^{\mathrm{m}}, 134$; al. $0^{\mathrm{m}}, 076-0^{\mathrm{m}}, 072$; caud. $0^{\mathrm{m}}, 068-0^{\mathrm{m}}, 063$; rostri culm. $0^{\mathrm{m}}, 016$; tarsi $0^{\mathrm{m}}, 017$.

Hab. in Papuasia - Ins. Salomonis, San Cristoval (Richards).

Obs. Monarcha M. castaneiventri, Verr. simitis, sed minor, sordidior, supra magis schistaceus, subtus pallidior et axillaribus omnino castaneis distinguendus.

La femmina di questa specie differisce da quella del $\boldsymbol{M}$. castanciventris, più che non differiscano i maschi, arendo colorito più chiaro e la gola non nera, ma grigia.

Mi sembra che questa specie appartenga al gruppo del $M$. inornatus, di cui sarebbe una forma molto più scura.

(262) Monarcha rubiensis (MEYER).

Monarcha rubiensis, Meyer, Sitzb. u. Abh. Gesellsch. Isis, 1884, Abh. I, p. 22 (ovo e nido).

\section{(263) Monarcha inornatus (GARN.).}

Monarcha inornatus, Sclat., Voy. Chall. Birds, p. 27 (1881) — Salvad., ibid., p. 65 (1881). Blas. u. Wehrk., Verh. zo-b. Gesellsch. Wien, 1882, p. 422 (Amboina)。 - Blas., P. Z. S. 1882, p. 706 (Ceram) - Sclat, P. Z. S. 1883, p. 347 (Fead Island, North of the Solomon Group) Pleske, Bull. Acad. Petersb. XXIX, p. 528 (Ternate) (1884). - Neyer, Sitzb. u. Abh. Gesellsch. Isis, 1884, Abh. I, p. 24. - Guillem., P. Z. S. 1885, p. 569 (Ternate).

Monarcha geelwinkianus, Meyer, Sitzb. u. Abh. Gesellsch. Isis, 1884, Abh. 1, p. 23 (Misori, Jobi) (tipo esaminato).

Monarcha fuscescens, Meyer, I. c. (Ins. Jamna); Id., Zeitsch. f. ges. Orn. 1886, p. 34 (tipo esaminato).

Monarcha commutatus, Meyer, I. c. p. 24.

Hab. in Ins. Mysori, Jobi, Jamna (Laglaize).

Senza volere negare la doruta importanza alle differenze distintive degli esemplari di Mysori, di Jobi e di Jamna, io non posso fare a meno di riferire quegli esemplari al $\boldsymbol{M}$. inornatus (Garn.), giacchè le differenze che essi presentano sono analoghe a quelle che sogliono presentare gli esemplari delle diverse località di questa specie, latamente diffusa, ed alle quali io ho gia accennato nella Omitologia $(\mathrm{I}, \mathrm{pp} .14,16)$. Aggiungeró qui che, avendo riceruto in comunicazione dal Meyer un esemplare tipico del suo $\boldsymbol{M}$. geelwinlianus di Ansus (Jobi) e tre del suo $\boldsymbol{M}$. $\boldsymbol{f u}$ scescens di Jamna, ho potuto constatare le seguenti differenze:

Il tipo del M. geelvinkianus si distingue pel colorito generale grigio più sudicio (o meno puro), pel colore bruno-cannella dell'addome più chiaro e pel colorito grigio della coda con lievissima tinta bruna, ma non pel colorito grigio del petto esteso più in basso, dipendendo quell'apparenza dal modo di preparazione della pelle.

Rispetto al $\boldsymbol{M}$. fuscescens, la descrizione del Meyer sembra fatta sul giovane anzichè sull'adulto, giacchè il carattere gula cinerea inferius sensim cinnamomeo tincta è proprio del giovane e non dell'adulto; i due caratteri più importanti, distintivi di questa forma sarebbero il colorito grigio-bruniccio delle ali e della coda, e le piume delle tibie grigio-cannella, e non di color cannella puro, come dice il Meyer. 
(264) Monarcha melanopsis (VIEILL.).

Monarcha melanopsis, Sharpe, Journ. Linn. Soc., Zool. XVI, p. 430 (Choqueri) (1882).

(265) Monarcha frater, Sclat.

Monarcha frater, Guillem., P. Z. S. 1885, p. 631 (Arfak)

(992) Monarcha periophthalmicus, ShaRpe.

Monarcha periophthalmicus, Sharpe, Gould's B. New Guin. pt. XIV, pl. 6 (1883). - Meyer Zeitschr. f. ges. Orn. III, p. 14 (1886).

(270) Monarcha nigrimentum, G. R. GR.

Monarcha nigrimentum, Blas. u. Nehrk., Verh. z.-h. Gesellsch. Wien, 1882, p. 422 (Amboina, (nido ed uova).

(272) Monarcha guttulatus (GARN.).

Piezorhynchus guttulatus, Sharpe, Journ. Linn. Soc., Zool. XVI, p. 431 (Choqueri) (1882).

Monarcha guttulatus, Meyer, Zeitschr. ges. Orn. I, p. 279 (1884) (nido e uova); III, p. 15 (1886), - Guillem., P. Z. S. 1885, p. 631 (Waigiou).

Il Meyer richiama l'attenzione sulla presenza di una, o di due serie di macchie bianche sulle cuopritrici delle ali degli esemplari della Nuova Guinea settentrivnale e della Nuova Guinea meridionale rispettiramente. Io credo piuttosto che quella differenza dipenda dall'età, giacchè quelle macchie mancano nei giovani.

(273) Monarcha vidua (Tristr.).

Piezorhynchus melanocephalus, Ramsay, Pr. Linn. Soc. N. S. W. IV, p. 468 (1879); VII, p. 24 (1882) (Isole Salomone).

Piezorhynchus vidua, Ramsay, Pr. Linn. Soc. L. S. W. VII, p. 23 (1882) (lsole Salomone). Tristr., Ibis, 1882, p. 142. - Sharpe, Gould's B. New Guin. pt. XVI, pl. 8 (1884).

(993) Monarcha squamulatus (Tristr.).

Piezorhynchus squamulatus, Ramsay, Pr. Linn. Soc. N. S. W. VII, pp. 42, 668 (1882). Sharpe, Gould's B. New Guin. pt. XVI, pl. 9 (1884).

Il Ramsay $(l . c$.$) confonde questa specie col suo P$. melanocephalus, che è identico. col $P$. vidua, Tristr.

(994) Monarcha ugiensis (RAMSAY).

Pomarea ugiensis, Ramsay, Pr. Linn. Soc. N. S. W. VII, p. 25 (1882: (Is. Satomone). - Sharpe Gould's B. New Guin. pt. XVII, pl. 6 (1884).

Monarcha ugiensis, Sclat, P. Z. S. 1883. p. 347.

Lo Sclater menziona cinque esemplari, tutti ugualmente colorati; egli conviene meco nel dovere riferire questa specie al genere Monarcha.

Sp. 1066 (275 bis) Monarcha buruensis, Merer.

Monarcha buruensis, Meyer, Sitzb. u. Abh. Gesellsch. Isis, 1884, Abb. I, p. 24 (1884) (Buru) (tipo. esaminato).

Monarcha pileatus, Meyer (nec Salvad.), ibid.

[198] 
Fronte, vertice, occipite, scapularibus et interscapularibus, dorso, alis caudaque nigris; gula media alba, nigro circumdata; capite in utraque parte, macula an. teoculari, auricularibus, torque nuchali, pectore, abdomine, supracaudalibus et subcaudalibus albis; pectore summo nigro lavato; tectricibus alarum minoribus nigris, apicibus albis, mediis albis, majoribus anterioribus albis, pogonio interno nigro notatis, posterioribus albis, primariarum tectricibus nigris; remigibus secundariis et tertiariis nigris, nonnullis pogonio externo et basi albis; axillaribus et subalaribus albis, nigrescenti variegatis; tibiis albis, nigro variegatis; rectricum extima dimidia ex parte, proxima apice, tertia pogonio interno macula apicali albis; rostro nigromargaritaceo (Meyer).

Long. tot. $0^{\mathrm{m}}, 145$; al. $0^{\mathrm{m}}, 071$; caud. $0^{\mathrm{m}}, 064$; rostri $0^{\mathrm{m}}, 013$; tarsi $0^{\mathrm{m}}, 017$ (ex Meyer).

$H a b$. in Moluccis - Buru (Riedel).

Dice il Meyer di aver ricevuto dal Riedel un esemplare, che egli crede differente specificamente dal $\boldsymbol{M}$. pileatus, Salvad. di Halmahera, giacchè dalla descrizione da me data di questa specie quell'esemplare differisce per avere le piccole cuopritrici delle ali non bianche, ma nere, pel bianco delle tre timoniere esterne, delle quali la prima ba bianco non solo l'apice, ma tutta la metà apicale, e per le dimensioni maggiori. Inoltre il Meyer fa notare che il suo esemplare differisce dalla descrizione che lo Sharpe (Cat. B. IV, p. 424) dà del M. pileatus, Salvad. per avere le scapolari nere e non bianche, per la fascia occipitale bianca non trasversale, per le piume tibiali bianche e nere, e non soltanto nere, e per le ascellari e cuopritrici inferiori delle ali bianche variegate di nero.

Ho esaminato il tipo di questa bella specie, che senza dubbio è diversa dal mio M. pileatus.

(276) Monarcha verticalis, ScLAT.

Monarcha verticalis, Sclat., P. Z. S. 1883, p. 347 (New Ireland and Duke of York Islands).

I due sessi sarebbero egualmente coloriti.

Sp. 1067 (276 bis) Monarcha castus, ScLAT.

Monarcha castus, Sclat., P. Z. S. 1883, pp. 51, 53, p. XlI, f. 1, p. 200.- Meyer, Sitzb, u. Abh. Gesellsch. Isis, 1884, Ahh. I, p. 25.

Piezorhynchus castus, Forbes, P. Z. S. 1884, p. 432. - Sharpe, Gould's B. New Guin. pt. XVI, pl. 7 (1884).

Supra niger; pileo et regione auriculari albis; fronte et taenia nucham cingente nigris; dorso summo taeniac nuchali proximo, uropygio et tectricibus alarum minoribus.cum scapularium marginibus externis albis; subtus albus, gutture nigro, maculis albis ornato; cauda nigra, rectricibus tribus externis albo late terminatis; subalaribus et remigum pogoniis internis albis; rostri plumbei tomiis albicantibus; pedibus plumbeis (Sclater).

Long. tot. $0^{\mathrm{m}}, 146$; al. $0^{\mathrm{m}}, 068$; caud. $0^{\mathrm{m}}, 068$; rostri culm. $0^{\mathrm{m}}, 014$; tarsi $0^{\mathrm{m}}, 019$.

Hab. in Papuasia - Ins. Tenimberensibus, Loetoe (Forbes, Riedel). 
Il Forbes ha raccolto un solo esemplare di questa specie, che egli trovò rara nella regione da lui esplorata; il Riedel ha inviato un secondo esemplare meno adulto, avente meno bianco sulla testa e sulle ali, e più sulla gola.

Questa specie, secondo lo Sclater, sarebbe affine al $M$. leucotis, Gould., dal quale differirebbe per avere la gola nera, e, secondo me, anche pel pileo bianco. Secondo lo Sharpe poi la specie più affine sarebbe il $M$. verticalis, Sclat., avendo anche il M. castus il pileo, la macchia auricolare ed i lati del collo bianchi, ma si distinguerebbe facilmente per le macchie bianche sulla gola e per le ultime remiganti nere marginate di nero e non interamente bianche.

(277) Monarcha infelix, ScLat.

Monarcha infelix, Sclat, Voy. Chall. Birds, p. 27, pl. VIII, ז. 1 (1881).

(278) Monarcha brodiei, Ramsay.

Piezorhynchus brodiei, Ramsay, Pr. Linn. Soc. N. S. W. VIII, p. 24 (1882) (I8ole Salomone). Tristr., Ibis, 1882, p. 142. - Sharpe, Gould's B. Nerw Guin. pt. XVIII, pl. 10 (1884). - Grant, P. Z. S. 1888, p. 195 ,

\section{Sp. 1068 (278 bis) Monarcha browni, RAMSAY .}

Monarcha (Piezorhynchus) browni, Ramsay, P. Z. S. 1882, p. 711. - Salvad., Orn. Pap. e Vol. III, p. 531 (1882).

Piezorhynchus browni, Sharpe, Gould's B. New Guin. pt. XVIIf, pl. 11 (1884).

Corpore supra, alis et cauda, gula et pectore summo nitide nigro-caeruleis; pectore imo, abdomine, axillaribus et subcaudalibus albis; rectricum extinarum quatuor tertia parte apicali alba; macula triangulari fere ab angulo oris in colli latere excurrente alba; plaga alba alari supra tectrices medias et apicem minorum; tectricibus minoribus albis uti dorsum; remigibus primariis et pogonio interno sccundariarum fusco-nigricantibus; alis subtus et parte basali plumarum cervicis et regionis interscapularis saturate fuscis; rostro nigro-caerulescente; pedibus caeruleo-plumbeis.

Long. tot. $0^{\mathrm{m}}, 177$; al. $0^{\mathrm{m}}, 087$; caud. $0^{\mathrm{m}}, 083$; rostri $0^{\mathrm{m}}, 017$; tarsi $0^{\mathrm{m}}, 021$.

Hab. in Papuasia - Ins. Salomonis, Marrabo (Brown).

Dice il Ramsay che questa bella specie è notevole per la grande area bianca che comincia sotto l'occhio, occupa alcune delle piume auricolari inferiori e si espande triangolarmente sui lati del collo, ma non giunge fino al petto; essa sarebbe affine. Piczorhynchus brodiei (Ramsay), ma sarebbe più grande ed arrebbe il nero del petto più esteso.

Nell'Appendice al volume III della mia Omitologia della Papuasia e delle Molucche, pag. 531, io menzionai questa specie senza poterne dare la descrizione, che ancora non era stata pubblicata.

\section{(282) Monarcha axillaris, SALvad.}

Monarcha axillaris, Meyer u. Finsch, Zeitschr. f. ges. Orn. 1886, p. 15.

Piezorhynchus axillaris, Sharpe, Gould's B. New Guin. pt. XXV, pl, 9 (1888).

$[200]$ 
Il Meyer fa notare che lo Sharpe descrive queste specie colle cuopritrici inferiori delle ali di un bianco puro, e tali egli le ha figurate anche recentemente, laddove io le descrissi soltanto cogli apici bianchi, come sono anche nell'esemplare esaminato dal Meyer. Le piume interamente candide sono le ascellari.

\section{(283) Monarcha chalybeocephalus (GARN.).}

Monarcha chalybeocephalus, Sharpe, Journ. Linn. Soc., Zool, XVI, p. 431 (East Cape) (1882). - Pleske, Bull. Acad. Petersb. XXIX, p. 528 (Ternate) (1881). - Nehrk., Journ. f. Orn. 1885, p. 32 (Waigeu). - Guillem., P. Z. S. 1885, p. 569 (Ternate, Batchian), 631 (Waigiou, Mysol).

Monarcha alecto, Finsch, Vög. d. Südsee, pp. 11, 25 (Nova Britannia, Port Moresby, Mabiak, Morilug, Cape York) (188\%. - Tristr., lbis, 1889, p. 556 (Joannet Island).

Hab. in insula Joannet dicta (Thomson).

(284) Monarcha nitidus (Gould).

Monarcha nitidus, Sclat., P. Z. S. 1883, pp. 51, 58, 194, 200 (Larat, Timor laut). - Forbes, P. Z. S. 1884, p. 432. - Neyer, Zeitschr. f. ges. Orn. I, p. 280 (1884) (nido ed uova).

Piezorhynchus nitidus, Ramsay, Tab. List, p. 6 (1888).

(288) Monarcha melanonotus, ScLAT.

Monarcha melanonotus, Nehrk., Journ. f. Orn. 1885, p. 32 (Waigeu). - Guillem., P. Z. S. 1885, p. 631 (Mysol, N. coast of Papua, Long. 139\%). Meyer, Zeitschr. f. ges. Orn. 1886, p. 34 (Kafu).

Dice il Meyer che gli esemplari di Kafu, tanto maschi quanto femmine, hanno le parti inferiori molto più vivacemente colorite degli esemplari della Nuova Guinea occidentale. La femmina ha inoltre la gola piu vivamente colorita e la testa tinta di color arancio scuro.

(289) Monarcha aruensis, SALVAD.

Monarcha aruensis, Meyer, Zeitschr. f. ges. Orn. I, p. 280 (1884).

Gen. HeteranaX,-Sharpe.

Heteranax, Sharpe, Gould's B. New Guin. pr. XVI (nel testo dell' $H_{0}$ mundus) I. mundus, Sclat.

\section{Sp. 1069 (289 bis) Heteranax mundus (Sclat.).}

Monarcha mundus, Sclat., P. Z. S. 1883, pp. 51, 54, pl. XII, f. 2, pp. 194, 200.

Heteranax mundus, Sharpe, Gould's B. New Guin. pt. XVI, pl. 10 (1884)。 - Forbes, P. Z. S. 1884, p. 432

Supra obscure cinereus; fronte lato, capitis lateribus et tectricibus alarum totis nigris; subtus albus; mento et plaga gulae media nigris; cauda nigra; rectricum quatuor lateralium apicibus latis albis; subalaribus albis; remigum pagina inferiore cinerea; rostro compresso, colore plumbeo, gonyde ascendente; pedibus migris (Sclater).

Long. tot. $0^{\mathrm{m}}, 162$; al. $0^{\mathrm{m}}, 082$; caud. $0^{\mathrm{m}}, 011$; rostri culm. $0^{\mathrm{m}}, 017$; tarsi $0^{\mathrm{m}}, 021$.

Hab. in Papuasia - Ins. Tenimberensibus, Larat (Forbes).

Questa specie, che per la colorazione sembra affine al $M$. nigrimentum, Gray, delle Molucche, viene considerata dallo Sharpe come tipo di un genere distinto, pel 
becco piuttosto lungo e compresso e col gonide ascendente. Non si conosce che un solo esemplare di questa specie ed esso fu raccolto dal Forbes nell'isola di Larat, non molto lungi dalla costa.

(290) Arses batantae, SHARPE.

Arses batantae, Nehrk., Journ. f. Orn. 1885, p. 32 (Waigeu). - Guillem., P. Z. S. 1885, p. 631 (Batanta, Waigiou).

Sp. 1070 (291 bis) Arses henkei, MEYET.

Arses telescophthalmus, Salvad. et D'Alb. (nec Garn.), Ann. Mus. Civ. Gen. VII, p. 819 (1875) (Nonte Epa). - Salvad., ibid. IX, p. 24 (1876) (Isola Yule, Naiabui), - Ramsay, Pr. Linn. Soc. ৯. S. W. HI, p. 375 (1878) (Cape York?); III, p. 114 (1878) (Baia Hall). - Sharpe, Journ. Pr. Linn. Soc. XIlI, pp. 316, 497 (1878) (Nicura, Laroki).

Arses (Ophryzone) telescophthalmus, Ramsay, Pr. I.inn. Soc. N. S. W. J, p. 391 (1876) (Port Moresby).

Arses enado, Ramsay (nec Less.), Pr. Linn. Soc. N. S. W. III, p. 269 (1879); IV, p. 98 (1879).

Arses aruensis, Sharpe, Journ. Linn. Sor., Zool. XIV, p. 631 (1879) (Port Moresby). - Salvad. Ann. Mus. Civ. Gen. XIV, p. 496, n. 30 (partim) (1879). - Id., Orn. Pap. e Mol. II, p. 47 (1887) (partim). - Finsch, Vög. d. Südsee, p. 26 (Laloki) (1884).

Arses henkei, Meyer, Zeitschr. f. ges. Orn. III, p. 16, Taf. III, f. 1, 2 (1886).

Mas. Mari A. aruensis, Sharpe, simillimus, sed mento tantum nigro distinguendus.

Foem. Foeminae A. telescophthalmae (Garn.) simitis, sed mento albo et tota a 77 idior.

Long. tot. $0^{\mathrm{m}}, 150$; al. $0^{\mathrm{m}}, 082-0^{\mathrm{m}}, 079$; caud. $0^{\mathrm{m}}, 078-0^{\mathrm{m}}, 080$; rostri culm. $0^{\mathrm{m}}, 012-0^{\mathrm{m}}, 013$; tarsi $0^{\mathrm{m}}, 016-0^{\mathrm{m}}, 017$.

$H a b$. in Papuasia - in Nova Guinea meridionali-orientali, Hall Bay ( $D^{\prime} A l$ bertis), ad flumen Laroki (Goldie), in montibus Astrolabii dictis (Hunstein).

Lo Sharpe fece già notare come forse gli esemplari della Nüova Guinea meridionale-orientale fossero specifieamente diversi dall' $A$. aruensis, ed io nella mia $O r-$ nitologia feci osservare che quegli esemplari erano intermedii all $A$. aruensis ed all'A. telescophthalmus. Recentemente il Meyer ha creduto di doverli considerare come specificamente diversi facendo notare come i maschi abbiano soltanto il mento nero, laddove nei maschi dell' $A$, aruensis e dell' $A$. telescophthalmus il nero si estende fino alla gola. La femmina poi si allontana da quella dell' $A$. aruensis più che non da quella dell' $A$. telescophthalmus e si avricina alquanto all' $A$. insularis (Meyer); essa differisce da quelle delle altre tre specie per avere il mento bianco e da quelle dell'A. aruensis e dell'A. telescophthatmus anche pel colorito bruno più chiaro.

Il Meyer afferma che l'A. hentiei differisce dall'A. aruensis più che non ne differisca l'A. telescophthalmus, la quale cosa non mi sembra al tutto esatta.

(292) Arses aruensis, SHARPF.

Arses aruensis, Meyer, Zeitschr. f. ges. Orn. I, p. 280 (1884) (nido ed uova).

\section{(293) Arses insularis (MEYER)}

Arses insularis, Guillem., P. Z. S. 1885, p. 632 (Jobi).

[202] 
(294) Sauloprocta melaleuca (Q. et G.).

Sauloprocta tricolor, Ramsay, Pr. Linn. Soc. L. S. W. VII, p. 23 (1882) (Isole Salomone). Finsch, Vög. d. Südsee, p. 12 (Niova Britannia) (1884).

Rhipidura tricolor, Tristr., Ibis, 1882, p. 142. - Grant, P. Z. S. 1887, p. 331 (Isole Salomone).

Sauloprocta melanoleuca, Meyer, Sitzb. u. Abh. Gesellsch. 1sis, 1884, Abh. I, p. 25 (nido ed uovo).

Sauloprocta melaleuca, Blas. v. Nehrk., Verh. z,-b. Gesulsch. Wien, 1882, p. 423 (Amboina, nido ed uova). - Pleske, BulJ. Acad. Petersb. XXIX, p. 528 (Ternate, nido ed uova) (1884). Tehrk., Journ. f. Orn. 1885, p. 32 (Waigeu). - Guillem.. P. Z. S. 1885, p. 570 (Ternate, Batchian), 632.

Sauloprocta motacilloides, Ramsay, Tab. List, p. 6 (1888).

\section{(295) Rhipidura cockerelli (RAMSAT).}

Sauloprocta cockerellii, Ramsay, Pr. Linn. Soc. X. S. W. VII, p. 23 (1882) (Isole Salomone).

Rhipidura cockerelli, Sharpe, Gould's B. New Guin. pt. XVII, pl, 5 (1884). - Grant, P. Z. S. 1888, p. 195.

Lo Sharpe conferma che questa specie non è una Sauloprocta e che appartiene al genere Rhipidura.

(296, 297) Rhipidura threnothorax, S. MüLL.

Rhipidura ambusta, Ramsily. - Salvad,, Orn. Pap. e Nlol. Il, p. 55 (1881); III, p. 531 (1882).

(301) Rhipidura leucothorax, Salvad.

Rhipidura leucothorax, Sharpe, Gould's B. lew Guin. pt. XVIII, pl. 9 (1884), - Meyer, Zeitschr. f. ges. Orm. 1886, p. 34 (Kafi1).

(302) Rhipidura obiensis, Salvad.

Rhipidura obiensis, Guillew., P. Z. S. 1885, p. 570 (0bi).

(305) Rhipidura setosa (Q. et G.).

Rhipidura setosa, Sharpe, Journ. Linn. Soc., Zool. XVI, p. 431 (Choqueri) (1882). - Nehrk., Journ. f. Orn. 1885, p. 32 (Waigeu). - Guillem., P. Z. S. 1885, p. 632 (Waigiou). - Ramsay, Tab. List, p. 6 (1888).

(306) Rhipidura assimilis, G. R. GR.

Rhipidura assimilis, Salvid, Voy. Chall. Birds, p. 68 (1881).

Sp. 1071 (307 bis) Rhipidura lenzi, Blas. (?).

Rhipidura sp., Lenz, Journ. f. Orn. 1877, p. 373 (Celebes!).

Rhipidura Lenzi, Blas, Journ. f. Orn. 1883, p. 145 (Celebes!). - Meyer, Sitzb. u. Abh. Gesellsch. Isis, I, p. 26 (1884). - Forbes, P. Z. S. 1884, p. 431 (Amboina).

Rhipidura cinereo-nigra, capite obscuriore; gula, striga suprcoculari conspicua lata, abdomine et subcaudalibus albis; pectore cinereo-plumbeo, maculis plumarum ovalibus albis mediis notato; alis fuscis, secundariis ad pogonium internum vix conspicue pallide marginatis; subalaribus cinereis, albo marginatis; cauda elongata, parum gradata, fusca, non albo terminata; rectricis extimae pogonio externo albo; rostro robusto, nigro; pedibus fuscis (Blasius).

Long. tot. $0^{\mathrm{m}}, 185$; al. $0^{\mathrm{m}}, 093$; caud. $0^{\mathrm{n}}, 090$; rostri $0^{\mathrm{m}}, 015$; tarsi $0^{\mathrm{m}}, 016$.

$\mathrm{Hab}$, in Moluccis - Amboina (Forbes). 
Questa specie, che da prima renne detta di Celebes, e poi dal Forbes fu indicata come propria di Amboina, ove egli ne aveva raccolto un esemplare, che ora trovasi nel Miseo Britannico, mi sembra non ancora ben definita.

Il Blasius dice che essa è intermedia alla $R$. cinerea, Wall. di Ceram ed alla $\boldsymbol{R}$. buruensis, Wall., ma pel colore bianco dell'addome e del sottocoda mi sembra più somigliante alla prima, che non alla seconda, che ha quelle parti rossigne; secondo il Blasius essa si distinguerebbe per avere la timoniera esterna marginata di bianco sul vessillo esterno, laddove la $R$. cinerea avrebbe la timoniera esterna unicolore. Non sono in grado di giudicare dell'importanza di quella differenza, che potrebbe dipendere dallo stato più, o meno fresco di quella timoniera.

La vicinanza di Amboina a Ceram e la comunanza delle specie nelle due isole mi fanno sospettare della identità della $R$. lenzi colla $R$. cinerea.

\section{Sp. 1072 (308 ${ }^{\text {bis }}$ ) Rhipidura fusco-rufa, ScLAT.}

Rhipidura fusco-rufa, Sclat., P. Z. S. 1883, pp. 194, 197, 200, pl. XXVIl. - Sharpe, Gould's B. New Guin. pt. XV, pl. 12 (1883). - Forbes, P. Z. S. 1884, p. 432. - Meyer, Zeitschr. f. ges. Om. I, p. 194 (1884). - Büttik., Not. Leyd. Mus. VIII, p. 62 (1886).

Supra obscure terreno-fusca, in dorso rufescenti tincta; alis nigricantibus, tectricum minorum apicibus et secundariorum marginibus externis late rufis; subtus rufa, mento et gutture toto ad medium pectus albis; subalaribus rufis; remigum marginibus internis fulvis; caudae nigricantis rectricibus tribus externis totis et paris proximi apicibus rufis; rostro et pedibus nigris (Sclater)..

Long. tot. $0^{\mathrm{m}}, 177$; al. $0^{\mathrm{m}}, 089$; caud. $0^{\mathrm{m}}, 089$; rostri $0^{\mathrm{m}}, 019$; tarsi $0^{\mathrm{m}}, 019$.

Hab. in Papuasia - Ins. Tenimberensibus, Larat, Moloe, Loetoe (Forbes).

Questa specie ha il becco largo e robusto, la coda poco graduata e senza dubbio, come fa notare lo Sclater, va annoverata nel gruppo che comprende la $R$. cinerca e la $R$. buruensis; essa si distingue facilmente da queste per le parti inferiori rossigne e per la coda colle timoniere esterne rossigne e le mediane nericcie.

Non so vedere la necessità d'includerla in una speciale divisione, come vorrebbe lo Sharpe.

Questa specie sembra comune nelle isole Tenimber, ove il Forbes ne ha raccolti molti esemplari; io ne ho esaminato uno raccolto dal Riedel ed inviato dal Dr. Meyer al Museo di Torino.

(309) Rhipidura hyperythra, G. R.

Rhipidura hyperythra, Sharpe, Gould's B. New Guin. pt. XXII, pl, 6 (1886).

(311) Rhipidura semirubra, Sclat.

Rhipidura semirubra, Sclat., Voy. Chall. Birds, p. 27, pl. VII, f. 2 (1881).

(312) Rhipidura russata, Tristr.

Rhipidura russata, Tristr., Ibis, 1882, p. 142. - Sharpe, Gould's B. New Guin. pt. XXI, nel testo della R. rubrofronlala (1886). - - Grant, P. Z. S. 1887, p. 331.

[204] 
Nell'Appendice al vol. III della mia Ornitologia, a pag. 532, seguendo l'opinione del Ramsay, io riunii questa specie alla $R$. rubrofrontata, Ramsay, ma lo Sharpe (l. c.) afferma che la $R$. russata è affatto distinta; sarebbe stato utile che egli aresse indicato quali sono le differenze.

\section{Sp. 1073 (312 bis) Rhipidura hamadryas, ScLAT.}

Rhipidura hamadryas, Sclat., P. Z., S. 1883, pp. 51, 54, 194, 200. - Sharpe, Gould's B. New Guin. pt. XV, pl, 11 (1883). - Forbes, P. Z. S. 1884, p. 432. - Meyer, Zeitschr. f. ges. Orn. I, p. 194 (1884).

Supra castanea, in capite postico ct cervice magis fuscescens; fronte dorso concolore; subtus pallide cervina; torque gutturali nigro; gula alba; alis caudaque nigricantibus; illis rufo anguste marginatis; hujus rectricibus externis cinerascentealbo late terminatis; rostro et pedibus nigris (Sclater).

Long. tot. $0^{\mathrm{m}}, 144$; al. $0^{\mathrm{m}}, 058$; caud. $0^{\mathrm{m}} 081$; rostri $0^{\mathrm{w}}, 012$; tarsi $0^{\mathrm{m}}, 020$.

Hab. in Papuasia - Ins. Tenimberensibus, Larat (Forbes).

Obs. Proxima R. dryadi, Gould, sed cervice postica rufescente nec fusca et alarum tectricibus rufo marginatis dignoscenda.

Lo Sharpe aggiunge che questa specie appartiene al gruppo delle specie del genere Rhipidura aventi il groppone ed il sopraccoda di color cannella, e che è affine alla R. semicollaris, S. Müll. di Timor, ed alla R. dryas, Gould, di Australia, dalle quali si distingue per avere la regione interscapolare rossigna come il dorso e per gli apici delle timoniere cinerei.

(314) Rhipidura rubrofrontata, RAMSAY.

Rhipidura rubrofrontata, Ramsay, Pr. Linn. Soc. N. S. W. VII, p. 23 (1881). - Sharpe, Gould's B. New Guin. pt. XXI, pl. 9 (1886). - Grant, P. Z. S. 1888. p. 195.

Dice lo Sharpe che questa specie, rappresentante nelle Isole Salomone la $R$. rufifrons (Lath.) dell'Australia, differisce da questa per essere più piccola, per avere gli apici delle timoniere di un bianco puro, e non bianco-cinereo, pel sottocoda di colore rossigno più cupo, per la fascia gutturale nera più stretta, per le piume auricolori più nere e pel colore rossigno alla base delle timoniere più ristretto e ricoperto dalle cuopritrici superiori della coda.

(316) Rhipidura squamata, S. MüLL.

Rhipidura squamata, Salvad., Voy. Ghail. Birds, p. 65, pl. XVIII, f. 2 (188I).

\section{Sp. 1074 (318 bis) Rhipidura opistherythra, ScLAT.}

Rhipidura opistherythra, Sclat,, P. Z. S. 1883, pp. 194, 197, 200. - Sharpe, Gould's R. New Guin. pt. XVI, pl. 4 (1884)。 - Forbes, P. Z. S. 1884, p. 432. - Meyer, Zeitschr. f. ges. 0rn. I p. 194 (1884).

Supra cineraceo-fusca; dorso postico castaneo-rufo; loris albidis; alarum nigricantium marginibus externis rufuscentibus; subtus pallide fulva; gutture albo, crisso castaneo, hypochondriis rufuscenti lavatis; caudae elongatae et valde gra- 
duatae rectricibus rufescentibus, supra castaneo extus marginatis; rostro superiore nigro, inferiore ad basin et pedibus pallidis (Sclater).

Long. tot. $0^{\mathrm{m}}, 170$; al. $0^{\mathrm{m}}, 086^{(1)}$; caud. rectr. med. $0^{\mathrm{m}}, 096^{(2)}$; rostri $0^{\mathrm{m}}, 015$; tarsi $0^{\mathrm{m}}, 025$.

Hab. in Papuasia - Ins. Tenimberensibus, Larat, Maroe (Forbes).

Questa specie va compresa in uno stesso gruppo colla $R$. rufidorsa, Meyer, della Nuova Guinea e di Jobi e colla $R$. rufa, Salvad., pure della Nuova Guinea, mancando anch'essa dello scudo pettorale nero; ma si distingue dalle altre per essere più grande, per non avere macchie distinte all'apice delle timoniere e per altre differenze.

\section{Sp. 1075 (319 bis) Rhipidura fallax, RAMSAx.}

Rhipidura fallax, Ramsiy, P. Z. S. 1884, p. 580.

Rhipidura cinnamomea, Meyer, Zeitschr. f. ges. Orn. 1886, p. 17, Taf. III, f. 3 (q).

Mas. Ater, vexillo interno remigum rectricumque fusco; apicibus axillarum tectricunque inferiorum alarum albis; rostro et pedibus nigris.

Long. tot. $0^{\mathrm{m}}, 139$; al. $0^{\mathrm{m}}, 076$; caud. $0^{\mathrm{m}}, 076$; rostri culm. $0^{\mathrm{nt}}, 015(3)$; tarsi $0^{\mathrm{m}}, 017$.

Foem. Cinnamomea, subtus vix pallidior; rectricibus mediis nigricantibus; primariis et secundariis supra fuscis, pogoniis externis cinnamomeis; remigibus subtus fuscis, laete cinnamomeo limbatis: maxilla obscura, mandibula albida (Meyer).

Long. tot. $0^{\mathrm{m}}, 135$; al. $0^{\mathrm{m}}, 073$; caud, $0^{\mathrm{m}}, 080$; rostri $0^{\mathrm{m}}, 010$; tarsi $0^{\text {m }}, 019$.

Hab. in Papuasia - Nova Guinea meridionali-orientali, in Montibus Astrolabii dictis (fide Ramsay), in Montibus Hufeisen (Hunstein).

Io non ho guari alcun dubbio che i due uccelli descritti dal Ramsay e dal Meyer siano maschio e femmina di una medesima specie, affine molto alla $R$. brachyrhyncha, Schleg, giacchè la femmina ( $R$. cinnanomea, Meyer) ha col maschio ( $R$. fallax, Ramsay) precisamente la stessa relazione che passa fra il maschio e la femmina della $R$. brachyrhyncha.

Il Ramsay fa notare che il maschio della $R$. fallax differisce da quello della R. brachyrhyncha per non avere traccia della macchia bianca sopraoculare, tanto cospicua nel maschio della $R$. brachyrhyncha; anche il Meyer fa notare che l'esemplare da lui descritto (senza dubbio una femmina, sebbene sia indicato come maschio) somigli alla femmina della $R$. brachyrhyncha, ma abbia colorito cannella più vivo e dimensioni minori.

(996) Rhipidura tenebrosa, RaMsay.

Rhipidura tenebrosa, Ramsay, Pr. Linu. S. N. W. VII, p̀, 33 (1881).

(320) Myiagra plumbea, V. et H.

Myiagra rubecula, Finsch, Vōg. d. Südsee, p. 35 (1884). - Ramsay, Tab. List, p. 6 (1888).

(1) Secondo lo Sharpe 0m,071.

(2) Secondo lo Sharpe 0m,101.

(3) Forse la lunghezza del becco non è esatta.

[206] 
(321) Myiagra galeata, G. R. Gr.

Myiagra galeata, Pleske, Bull. Acad. Petersb. XXIX, p. 529 (Ternate) (1884;

(323) Myiagra ruficollis (VIEILL.).

Myiagra ruficollis, Meyer, Zeitscbr. f. ges. Orn. 1, p. 281, Taf. XVII, t. 6 (ovum) (1884).

Il Meyer descrive parecchi nidi e le uova trovati dal Ribbe nelle isole Aru; alcuni nidi contenevano uova di Cuculi.

\section{Sp. 1076 (324 bis) Myiagra fulviventris, ScLat.}

Myiagra fulviventris, Sclat, P. Z. S. 1883, pp. 51, 52, 54, 195, 200. - Forhes, P. Z. S. 1882, p. 432. - Neyer, Zeitschr, f. ges. Orn. I, P. 194 (1884). - Sharpe, Gould's B. New Guin. pt. XIX, pl. 13 (1885). - Büttik., Not. l.eyd. Mus. VIII. p. 62 (1886).

Supra plumbea, capite et dorso nitore caeruleo tinctis; alis et cauda fusconigricantibus, subtus saturate castaneo-rufa; abdomine et subalaribus fulvis; remigum marginibus interioribus albicantibus; rostro et pedibus nigris (Sclater).

Foem. Paullo minor et pallidior.

Long. tot. $0^{\mathrm{m}}, 139$; al. $0^{\mathrm{m}} ; 068$; caud. $0^{\mathrm{m}}, 068$; rostri culm. $0^{\mathrm{m}}, 014$; tarsi $0^{\mathrm{m}}, 019$.

Hab. in Papuasia - Ins. Tenimberensibus, Larat (Forbes).

Dice lo Sharpe che questa specie somiglia alla femmina della II. gateato, Gray, ma che ne differisce per avere le parti superiori di un grigio-plumbeo più scuro e per la gola di color rossigno-castagno molto vivo.

(326-327) Myiagra ferrocyanea, RAMSAY.

Myiagra pallida, Ramsay, Pr. Linn. Soc. N. S. W. VII, p. 24 (1882) (Isole Salomone), - Salvad,, Orn. Pap. e Mol. JJ, p. 29 (1881).

Myiagra ferrocyanea, Ramsay, Pr. Linn. Soc. X. S. W. VII, p. 24 (1882) (Isole Salomone). -

Tristr., Ibis, 1882, p. 142. - Sharpe, Gould's B. New. Guin. nt. XVII, pl. 8 (1884). - Grant, P. Z. S. 1888 , p. 142 .

L'Ogilvie-Grant afferma che la $M$. pallida, Ramsay, non è altro che la femmina di questa specie, come era già stato sospettato.

(328) Myiagra cervinicauda, Tristr.

Myiagra cervinicauda, Mamsay, Pr. Linn. Soc. X. S. W. VII, p. 24 (1882). - Sharpe, Gould's B. New Guin. pt. XVIII, pl. 6 (1884).

Lo Sharpe fa notare che il maschio di questa specie, somigliante a quello della II. ferrocyanea, ne differisce per avere il nero delle parti superiori con riflessi verdebottiglia, laddove in quello il nero ha riflessi porporini.

(329) Muscicapa griseosticta (SwiNH.).

Muscicapa griseosticta, Blas,, P. Z. S. 1882, p. 706 (Ceram.). - Pleske, Bull. Acad. Petersb. XXIX, p. 529 (Ternate) (1884) - Guillem., P. Z. S. 1885, p. 632 (Waigiou, Mysol).

\section{(332) Monachella saxicolina, SALvad.}

Monachella mulleriana, Guillem., P. Z. S. 1885, p. 632 (Arfak). 


\section{(343) Poecilodryas bimaculata (SALVAD.).}

Poecilodryas melanoleuca, Ramsay, Abstr. of Pr. Linn. Soc. N. S. W., 31 Jan. 1883, p. 3. Poecilodryas sylvia, Ramsay, Pr. Linn. Soc. N. S. W. VIII, p. 19 (1883). - Salvad, Ibis, 1884, p. 353. - Sharpe, Gould's B. Jew Guin. pt. XVI, pl. 6 (1884).

Hab. in Nova Guinea meridionali orientali, in Montibus Astrolabii (Goldie). Lo Sharpe ha figurato il tipo della $P$. sylvia, Ramsay.

(335) Poecilodryas hypoleuca (G. R. GR.)

Poecilodryas hypoleuca, Meyer, Sitzb. u. Abh. Gesellsch. Isis, 1884, Abh. I, p. 27 (Salwatti, Laglaize). - Guillem., P. Z. S. 1885, p. 632 (Salwatti, Waigiou).

\section{Sp. 1077 (335 bis) Poecilodryas minor (MEYER) (?).}

? Myiolostes minor, Bernst., in Musschenbr., Dagboek, pp. 113, 114 (Norong) (1883).

Poecilodryas minor, Meyer, Sitzb. u. Abh. Gesellsch. Isis, 1884, Abh. I, p. 27 (Nova Guinea occ. et Salrwatti).

Pocilodryas P. hypolancae similis, sed minor, superne obscure fusca et macula pectorali genisque nigrescentibus (Meyer).

Long. tot. $0^{\mathrm{m}}, 115-0^{\mathrm{m}}, 110$; al. $0^{\mathrm{m}}, 070-0^{\mathrm{m}}, 060$; caud. $0^{\mathrm{m}}, 051-0^{\mathrm{m}}, 049$; rostri $0^{\mathrm{m}}, 014-0^{\mathrm{m}}, 013 ; \operatorname{tarsi} 0^{\mathrm{m}}, 015-0^{\mathrm{m}}, 014$.

Hab. in Papuasia - Nova Guinea occidentali (Dorey, Samsom, Karons) et ins. Salwatti (Laglaize).

Secondo il Meyer, oltre che per le dimensioni minori e per la minore robustezza del becco, questa specie si distingue pel colorito delle parti superiori volgenti al bruniccio e più uniforme, mentre nella $P$. hypoleuca la testa è più oscura del dorso.

Io ho esaminato uno dei suoi tipi dei Karons; esso corrisponde, tranne che nelle dimensioni un poco minori, con una femmina di Dorei-Hum, raccolta dal Beccari, da me attribuita, insieme con due maschi notevolmente più grandi (Orn. I, p. 86); alla $P$. hypoleuca. La bontà specifica della $P$. minor, Meyer, non mi sembra fuori di ogni dubbio.

\section{(337) Poecilodryas pulverulenta (MüLL.).}

Eopsaltria pulverulenta, Gadow, Cat. B. VIII, p. 180 (1883).

Poecilodryas pulverulenta, Finsch. u. Meyer, Zeitschr, f. ges. Orn. 1886, p. 17 (Aroani).

Eopsaltria leucura, Ramsay, Tab. List, p. 7 (1888).

\section{(338) Poecilodryas cyana (Salvad.).}

Poecilodryas cyana, Gadow, Cat. B. VIII, p. 182 (nota 2) (1883). - Finsch u. Meyer, Zeitschr. f. ges. Orn. 1886, p. 17 (Monti Hufeisen).

\section{(997) Poecilodryas albifacies (SHARPE).}

Poecilodryas albifacies, Ramsay, Pr. Linn. Soc. N. S. W. VIII, p. 25 (1883) (nido ed uova). Finsch u. Meyer, Zeitschr. f, ges. Orn. 1886, p. 12 (Monti Hufeisen).

Il Meyer cosi descrive un esemplare in abito di transizione: Parti superiori brune, variegate di olivaceo sul dorso; il bianco della faccia e del mento in parte tinto bruno; parti inferiori brune, rariegate di giallo. 
Genus ERYTHROMYIAS, Sharpe.

Erythromyias, Sharpe, Cat. B. Brit. Nus. IV, p. 199 (1879) - . Saxicola dumeloria, Wall.

\section{Sp. 1078 (341 bis) Erythromyias (?) riedeli, Büттік.}

Erythromyas Riedeli, Büttik., Not. Leyd. Mus. VIII, p. 62, pl. 3, f. 1 (1886).

Supra olivaceo-fusca, pileo, nucha et capitis lateribus olivascente-nigris; stria superciliari lata a naribus ad nucham ducta et nucham cingente isabellina; dorso olivaceo, interscapuliis pallide fulvo stratis, cauda supracaudalibusque ferrugineis, rectricibus sub certa luce fusco transfasciolatis; remigibus nigricantibus, primariis extus acute, secundariis latius, tertiariis late fulvo marginatis, scapularibus et tectricibus alarum minoribus dorso concoloribus, mediis late fulvo terninatis; subalaribus pallide fulvis; subtus fulvide (!) rufa, gula pallidiore, mento, abdomine et subcaudalibus albis, plus minusve fulvido tinctis; tibiis nigro-fuscis; rostro nigro; pedibus pallidis (Büttikofer).

Long. tot. $0^{\mathrm{m}}, 105 ;$ al. $0^{\mathrm{m}}, 061$; caud. $0^{\mathrm{m}}, 040$; tarsi $0^{\mathrm{m}}, 020$ (Büttikofer).

Hab. in Papuasia - Insulis Tenimberensibus (Riedel).

Ho riferito la descrizione data dal Büttikofer. Giudicando dalla figura, pare che questa specie somigli alla E. pyrrhonota (S. Müll.), di Timor, dalla quale differisce per molti rispetti, per essere più grande, per la fascia sopraccigliare e per le parti inferiori fulve, per mancare della larga fascia nera sul petto e per altre differenze; $\mathrm{ma}$ io sospetto che, piuttosto che al genere Erythromyias, essa possa appartenere al genere Poecilodryas, sembrandomi di scorgere noteroli somiglianze fra la specie del Buttikofer e la $P$. brachyura (Sclat.).

Dice il Büttikofer di aver ricevuto dal Riedel un solo esemplare, senza indicazione del sesso e piuttosto in cattivo stato.

(342) Microeca flavovirescens, G. R. Gr.

Microeca flavovirescens, Sharpe, Journ. Linn. Soc., Zool. XVI, p. 432 (Morocco) (1882). - Finsch, Vög. d. Südsee, p. 26 (Laloki), 35 (1884)。 - Nehrk., Jourı. f. Orn. 1885, p. 32 (Waigeu). - Guillem., P. Z. S. 1885, p. 632 (Johi).

Microura (errore?) flavovirescens, Bernst., in Musschenbr., Dagboek, p. 149 (Sorong) (1883). Microeca flavovirens (errore), Bernst., op. cit. p. 159 (Kalwal) (1883).

(343) Microeca flaviventris, Salvad.

Microeca flavigaster, Ramsay, Tab. List, p. 6 (1888).

\section{Sp. 1079 (344 bis) Microeca hemixantha, Sclat.}

Microeca hemizanta, Sclat., P. Z. S. 1883, pp. 51, 55, 195, 200 - Forbes, P. Z. S. 1884, p. 432。 Meyer, Zeitschr. f. ges. Orn. I, p. 194 (1884). - Büttik., Not. Leyd. Mus. VIII, p. 62 (1886).

Supra flavicanti-olivacea; alis caudaque fuscis, dorsi colore marginatis, loris et linea supercitiari obsoleta flavidis; macula auriculari fusca; subtus flava, remigum marginibus internis albidis: subalaribus flavis; rostri fusci mandibula inferiore pallida; pedibus nigris (Sclater). 
Long. tot. $0^{\mathrm{m}}, 122 ;$ al. $0^{\mathrm{nd}}, 073$; caud. $0^{\mathrm{m}}, 053$.

Hab. in Papuasia - Ins. Tenimberensibus, Larat, Loetoe (Forbes).

Lo Sclater non indica a quale specie congenere questa più somigli; egli si contenta far notare che essa ha il colorito della Poecilodryas papuana, la quale cosa si può dire anche della Microeca laeta, Salvad.

Io ho esaminato un esemplare raccolto dal Riedel ed inviato dal Meyer al Museo di Torino; esso ha dimensioni alquanto minori di quelle indicate dallo Sclater: al. $0^{\mathrm{m}}, 070$; coda $0^{\mathrm{m}}, 050$; becco $0^{\mathrm{m}}, 010$; tarso $0^{\mathrm{m}}, 015$. Mi sembra che questa specie sia affine alla $\boldsymbol{M}$. laeta, Salvad., dalla quale si distingue facilmente per non avere le piume più lunghe del sopraccoda rossigne, per mancare delle macchie bianchiccie all'apice delle grandi cuopritrici delle ali e pel becco meno largo.

Genus GERYGONE, Gould.

Typus:

Leptotodus, Meyer, Zeitschr. f. ges. Orn. I, p. 192 (1884)

Microlestes, Meyer, Zeitschr. f. ges. Orn. I, p. 197 (1884)
L. Lenuis, Meyer.

M. arfakianus, Meyer.

\section{Sp. 1080 (345 bis) Gerygone dorsalis, ScLat.}

Gerygone dorsalis, Sclat., P. Z. S. 1883, pp. 185, 199, 200. - Sharpe, Gould's B. New Guin. pt. XVI, pl. 2 (1884). - Forbes, P. Z. S. 1884, p. 432. - Meyer, Zeitschr. f. ges. Orn. I, p. 194 (1884).

Supra brunnescenti-castanea, alis caudaque nigris, dorsi colore limbatis, piteo et nucha murino-fuscis; subtus alba; hypochondriis rufescenti lavatis; subalaribus albis; caudae rectricibus subtus in pogoniis interioribus nigricantibus, macula versus apicem alba praeditis; rostri et pedibus nigris; iride nigra (Sclater).

Foem. Mari similis.

Long. tot. $0^{\mathrm{m}}, 101$; al. $0^{\mathrm{m}}, 053$; caud. $0^{\mathrm{m}}, 040$; rostri culm. $0^{\mathrm{m}}, 014$; tarsi $0^{\mathrm{m}}, 020$.

Hab. in Papuasia - Ins. Tenimberensibus, Larat, Loetoe, Meloe (Forbes).

Io ho esaminato il tipo di questa specie, che mi fu inviato dallo Sclater; essa differisce da tutte le altre pel colore rossigno-castagno delle parti superiori, che contrasta col colore grigio del pileo.

\section{Sp. 1081 (345 ter) Gerygone fulvescens, MEYER.}

Gerygone fulvescens, Meyer, Sitzb. u. Abh. Gesellsch. Isis, 1884, Abh. I, p. 27 (1884).

Gerygone G. dorsali similis, sed supra fulvescens, remigum rectricumque pogoniis externis pallide olivascenti anguste limbatis (Meyer).

Long. tot. $0^{\mathrm{m}}, 096$; al. $0^{\mathrm{m}}, 054$; caud. $0^{\mathrm{mm}}, 041$; rostri $0^{\mathrm{m}}, 010$; tarsi $0^{\mathrm{m}}, 022$.

Hab. in Papuasia - Ins. Babbar ad occasum ins. Timor-laut (Riedel).

Ho esaminato il tipo di questa specie, che, come fa notare il Meyer, differisce dalla $G$. dorsalis, Sclat. pel colorito meno vivace, il colore bruno essendo tinto di grigiastro, rolgente all'olivaceo; le dimensioni sono quasi eguali, forse un po' maggiori nella $G$. fulvescens. 
(316) Gerygone palpebrosa, Wall.

Pseudo-gerygone palpebrosa, Meyer, Zeitschr. f. ges. Orn. I, p. 282 (1884).

(347) Gerygone personata, Goold.

Gerygone (Pseudo-gerygone) personata, Ramsay, Tah. List, p. 7 (1888).

(348) Gerygone chrysogaster, GraY.

Gerygone chrysogaster, Neyer, Zeitschr. f. ges. Orn. I, p. 282 (1884)

Pseudo-gerygone chrysogaster, Sharpe, Gould's B. llew Guin. pt. XXII, pl. 12 (1886).

\section{SP. 1082 ( $348^{\text {bis }}$ ) Gerygone cinereiceps (SHARPE).}

Pseudo-gerygone cinereiceps, Sharpe, Nature, vol. 34, p. 340 (Aug. 12, 1886). - Id., in Gould's B. New Guin. pt. XXII, pl. 13 (1886).

Supra viridi-flavescens, supracaudalibus magis olivaceis; tectricibus alarum majoribus, remigibus rectricibusque fuscis, dorsi colore marginatis, rectricibus fascia subapicali nigra, macula apicali fiesco-grisea, altera parva in pogonio interno alba notatis; pileo cinereo vix viridi tincto; loris albis; genis et plumis auricularibus fusco-griseis; gula et corpore subtus in medio albis, lateribus pectoris fusco-cinereis, lateribus abdominis flavo-sulphureis; subcaudalibus albis vix flavo tinctis; subalaribus et axillaribus albis, flavo marginatis; remigibus subtus fuscis, intus albo marginatis: rostro nigro; pedibus grisco-lilacinis; iride rubra.

Long. tot. $0^{\mathrm{m}}, 090$; al. $0^{\mathrm{m}}, 048$; caud. $0^{\mathrm{m}}, 031$; rostri $0^{\mathrm{m}}, 010$; tarsi $0^{\mathrm{m}}, 016$.

Obs. Similis P. flavilaterali, Gray, ex Nova Caledonia, sed minor et rectricibus haud subterminaliter albofasciatis distinguenda (Sharpe).

Hab. in Papuasia - Nova Guinea meridionali-orientali, in Montibus Astrolabii (Forbes).

\section{(351) Gerygone notata, Salvad.}

Leptotodus tenuis, Meyer, Zeitschr. f. ges, Orn. I, p. 197 pl. IX, f. 2 (1884) (Amberbaki). Pseudo-gerygone notata, Sharpe, Gould's, B. New Guin. pt. XXI, pl. 13 (1886).

Ho esaminato il tipo del Leptotodus tenuis, Meyer, che senza dubbio è identico colla G. notata, Salvad.

(355) Gerygone brunneipectus, SHARPE.

Gerygone magnirostris, part., Ramsay, T'ab, List, p. 6 (1888).

(358) Gerygone arfakiana, Salvad.

Microlestes arfakianus, Meyer, Zeitschr. f. ges. Orn. I, p. 198 (1884) (Arfak).

Ho esaminato il tipo del Microlestes arfatianus, che ha il becco guasto e deformato per schiacciamento ed apparentemente più lungo della realtả per mancanza di piume alla base; esso somiglia moltissimo ai tipi della Gerygone arfakiana, cui credo che debba essere riferito, sebbene abbia l'ala 3 millimetri e la coda 4 millimetri più lunghe. 


\section{Sp. 1083 (361 bis) Gerygone bimaculata, MEYrr.}

Gerygone bimaculata, Meyer, Zeitschr, f. ges. Orm. I, p. 198 (Arfak) (1884).

Supra pallide griseo-brunnea, fronten versus rufescens; subtus alba, pectore mufescenti, corporis lateribus brunnescenti tinctis; alis fuscis, remigibus anguste albescenti marginatis, subataribus et subcaudalibus albis; canda fusca cum fascia lata subapicali nigra notata; rectricibus, dualus mediis exceptis, macula subapicali alba, valde conspicua in pogonio interno notatis; rectricum ultima in pogonio externo quoque; rostro pedibusque nigris (Meyer).

Long. tot. $0^{\mathrm{m}}, 084$; al. $0^{\mathrm{m}}, 048-0^{\mathrm{m}}, 051$; caud. $0^{\mathrm{m}}, 034-0^{\mathrm{m}}, 035$; rostri culm. $0^{\mathrm{m}}, 07$; tarsi $0^{\mathrm{m}}, 015$.

Hab. in Papuasía - in Nova Guinea, Montibus Arfak (Laglaize).

Il Meyer menziona due femmine, e dice che questa specie è affine alla G. ruficollis, Salvad., ma che ne differisce per avere la timoniera esterna con una macchia bianca anche nel vessillo esterno, per la quale cosa il Mejer l'ha chiamata bimaculata; inoltre manca della linea sopraccigliare bianca; la gola e la parte anteriore del collo sono bianche e non rossigne e soltanto sul petto appare una tinta bruniccia, laddove il resto delle parti inferiori sono bianche.

Ho esaminato il tipo di questa specie affine, che sembra distinta dalla G. ruficollis, Salvad.

(364) Machaerorbynchus xanthogenys, G. R. GR.

Machaerorhynchus, xanthogenys, Salvad., Voy. Chall., Birds, p. 78 (1881). Machaerorhynchus flaviventer, Ramsay 'nec Gould), Tah. List, nota 171 (1888).

(365) Machaerorhynchus albifrons, (G. R. Gr.).

Machaerorhynchus albifrons, Nehrl., Journ. f. Orn. 1885, p. 32 (Waigeu). - Guillem., P. Z. S. 1885, p. 633 (Arfak).

(366) Machaerorhynchus nigripectus, ScHLEG.

Machaerorhynchus flaviventer, Ramsay (nec Gould), Pr. Linn. Soc. N. S. W. IV, p. 90, 97 (1879) (Goldie River).

Machaerorhynchus nigripectus, Guillem., P. Z. S. 1885, p. 633 (Arfak). - Finsch u. Meyer, Zeitschr. f. ges. Orn. 1886, p. 18 (Monti Hufeisen).

Il Meyer menziona una femmina della Nuova Guinea meridionale-orientale che egli mi ha gentilmente inviato affinchè l'esaminassi; essa corrisponde colle femmine dei Monti Arfak, tranne che ha il giallo delle parti inferiori più intenso, pel quale rispetto somiglia ai maschi, dai quali pure differisce per la macchia nera sul petto più piccola. Converrà attendere un maggior numero di esemplari per decidere se quelli della Nuova Guinea meridionale-orientale appartengano ad una specie distinta dal M. nigripectus, Schleg.

(370) Todopsis bonapartei, G. R. Gr.

Todopsis bonapartei, Meyer, Zeitschr. f. ges. Orn. I, p. 282 (1884). [212] 
(373) Todopsis coronata, Gould.

Todopsis coronata, Meyer, Zeitschr, f. ges, Orn. I, p. 282 (1884).

Il Meyer menziona due esemplari delle isole Aru (Ribbe), differenti da uno della T. wallacei, Gr. di Ramo, per avere le macchie azzurre del pileo più scure e più rivaci (?), e pel becco e per le ali più lunghe.

\section{(374) Malurus alboscapulatus, MEYER.}

Malurus alboscapulatus, Sharpe, Journ. Linn. Soc., Zool. XVI, p. 433 (Choqueri) (1882)。 Guillem., P. Z. S. 1885, p. 633 (Arfak).

(375) Graucalus caeruleogriseus (G. R. Gr.).

Artamides caeruleogriseus, Sharpe, Journ. Linn. Soc., Zool. XVI, p. 433 (1882).

Graucalus caeruleogriseus, Guillem., P. Z. S. 1885, p. 633.

Il Guillemard fa notare che i tre esemplari della sua collezione, di Mansinam, di Dorei e del Monte Arfak, tutte tre femmine, hanno le parti inferiori piủ scure degli esemplari delle isole Aru nel Museo Britannico.

\section{SP. $1084\left(375^{\text {bis }}\right)$ Graucalus unimodus, ScLAT.}

Graucalus unimodus, Sclat., P. Z. S. 1883, pp. 51, 55, 195, 198, 200. - Mejer, Zeitschr. f. ges. Urn. I, p. 194 (1884! -- Büttik., Not. Leyd. Mus. VIII, p. 63 (1886).

Artamides unimodus, Sharpe, Gould's B. New. Guin. pt. XVI, pl. 17 (1884). - Forbes, P. Z. S. 1884, p. 432.

Cinereo-ardesiacus; fronte, Toris et capitis lateribus cum gutture toto ad medium pectus aeneo-nigris; alis et cauda nigris, illis cinereo exterius marginatis; subalaribus pallide isabellinis in medio griseo-violaceis; remigum pagina inferiore albicanti-cinerea; rostro et pedibus nigris; iride fusco-brunnea.

Foem. Mari similis, sed paullo pallidior et colore nigro nisi in loris carens; subalaribus latius isabellinis (Sclater).

Long. tot. $0^{\mathrm{m}}, 345 ;$ al. $0^{\mathrm{m}}, 194$; caud. $0^{\mathrm{m}}, 166$; rostri culm. $0^{\mathrm{m}}, 031 ; \operatorname{tarsi} 0^{\mathrm{m}}, 029$.

Hab. in Papuasia - Ins. Tenimberensibus (Forbes, Riedel).

Questa specie somiglia al Gr. caeruleogriseus, Gr. delle isole Aru e della Nuova Guinea, arendo le cuopritrici inferiori delle ali isabelline, ma ne differisce pel colorito grigio-schistaceo e non azzurrognolo, pel nero esteso su tutta la parte anteriore del collo nel maschio; anche la femmina si distingue per avere il margine frontale e le redini nere e non di color grigio-chiaro azzurrognolo. Forse questa specie è più affine al $G r$. pollens, Salvad., delle isole Kei, dal quale differisce pel colore isabellino delle cuopritrici inferiori delle ali.

Io ho esaminato due esemplari di questa specie, raccolti dal Riedel ed inviati dal IIejer al Museo di Torino; la femmina ha le piume del sottocoda marginate di rossigno all apice e le cuopritrici delle remiganti primarie cogli apici bianchicci isabellini. 
(376) Graucalus boyeri (G. R. Gr.).

Graucalus boyeri, Guillem., P. Z. S. 1885, p. 633 (Wysol).

(378) Graucalus pollens, SALrad.

Graucalus polleas, Salvad, Voy. Chall., Birds, p. 68, pl. XIX (1881).

(379) Graucalus parvulus, Salvad.

Graucalus parvulus (?), Meyer, Sitzb. u. Alh. Gesellsch. Isis, 1884, Abh. I, p. 27.

Il Meyer menziona un esemplare d'incerta località, conservato nel Museo di Dresda, e che egli considera come femmina di questa specie; dal maschio differisce per la mancanza di nero sulla testa e sulla gola; esso è di un bel color grigioazzurro. Il Meyer dice che se non è la femmina del G. parvulus, appartiene ad una specie non ancor descritta. Quell'esemplare è stato confrontato dal Dott. Jentink coi tipi del G. parvulus, nel Museo di Leida, ed egli afferma che corrisponde con quelli, tranne che manca del nero della testa; il colorito sarebbe quasi lo stesso. Siccome i tipi della mia specie non avevano punto colorito azzurrognolo, io penso che l'esemplare del Museo di Dresda appartenga ad altra specie.

(381) Graucalus magnirostris, Forsten.

Graucalus magnirostris, Guillem., P. Z. S. 1886, p. 570 (Batchian), 633 (Waigieud). - Salvar., Ibis, 1886, p. 154 .

L'esemplare indicato di Waigiou senza dubbio deve essere di Batchian.

\section{(382) Graucalus melanops (LATH.).}

Graucalus melanops, Salvad., Voy. Chall., Birds, p. 78 (1881). - Meyer, Verh. z.-b. Ges. Wien, 1881, p. 771 (Luang). - Sclat., P. Z. S. 1883, pr. 51, 195, 200 (Timor-laut, Forbes). - Forbes, P. Z. S. 1882, p. 432. - Guillem., P. Z. S. 1885, p. 63 ' (Mysol:- Büttik., Not. Leyd. Mus. VIII, p. 63(1886). - Ramsay, Tab. List, p. 4 (1888).

\section{(384) Graucalus papuensis (GM.)}

? Ceblepyris melanolora, Bernst., in Musschenhr. Dagboek, p. 153 (Sailolo) (1883).

Graucalus papuensis, Pleske, Rull. Acad. Petersb. XXIX, 1. 529 (Ternate)(1884). - Guillem., P. Z. S. 1885, p. 634 (Mysol, Salwatti, Jobi).

(385) Graucalus sclateri, FINsc日.

Graucalus Sclateri, Finsch., Vög. d. Südsee, p. 12 (Nova Britannia) (1884).

\section{(386) Graucalus hypoleucus, Gould.}

Graucalus elegans, Ramsay, Pr.jLinn. Soc. N. S. W VII, p. 22 (1882). - (irant, P. Z. S. 1887, p. 331. Graucalus hypoleucus, Tristr., Ibis, 1882, p. 142. - Sharpe, Journ. Linn. Soc., Zool. XVI,p. 433 (Taburi) (1882). - Finsch, Vög. d. Sildsee, p. 35 (1884).

? Graucalus sclateri, Grant (nec Finsch), P. Z. S. 1888, p. 196 (Aola, Guadalcanar).

Graucalus hyperleucus (sic), Ramsay, Tab. List, p. 4 (1888).

Dice l'Ogilvie-Grant che un maschio adulto di Fauro corrisponde bene colla descrizione del Gr. clegans del Ramsay; io ho espresso l'opinione (Orn. III, p. 534) che 
questo sia identico col Gr. hypoleucus. Il Gr. sclateri, Finsch, è della Nuova Britannia, e mi pare difficile che questa specie si trovi anche nelle isole Salomone, e quindi, a meno che gli esemplari di queste isole non siano riferibili al Gr. hypoleucus, Gould, probabilmente appartengono ad una specie innominata.

\section{SP. 1085 (386 bis) Graucalus timorlaoensis, MEYER.}

Graucalus timorlaoensis, Meyer, Zeitschr. f. ges. Orn. 1, p. 199, Taf. IX, f. 1 (1884) (Timorlaut) (Tipo esaminato'.

Grancalus G. Hypolenco, Gould, simillimus, sed minor et pectore albo distinguendus.

Supra cinereus; fronte alba, loris et regione suboculari nigrescentibus; subtus albus; remigibus fusco-cinereis, albo limbatis; rectricibus duabus mediis fusco-cinereis, reliquis nigrescentibus, plus minusve albo limbatis; rostro pedibusque nigris (Meyer).

Long. tot. $0^{\mathrm{m}}, 235$; al. $0^{\mathrm{m}}, 140-0^{\mathrm{m}}, 145$; caud. $0^{\mathrm{m}}, 108-0^{\mathrm{m}}, 113$; rostri culm. $0^{\mathrm{m}}, 023-0^{\mathrm{m}}, 024 ;$ tarsi $0^{\mathrm{m}}, 011-0^{\mathrm{m}}, 012$.

Hab. in Papuasia - Ins. Timor-laut (Riedel).

Il Meyer mi ha cortesemente inviato, affinchè li esaminassi, i due esemplari tipici, che sono probabilmente ambedue femmine.

Come fa notare il Meyer, questa specie è affine al Gr. hypoleucus, dal quale differisce per avere le parti inferiori di un bianco puro, ed anche per le dimensioni notevolmente minori. Le remiganti secondarie hanno esternamente larghi margini bianchi, i quali formano sull'ala chiusa una fascia longitudinale; le timoniere mediane sono di color grigio scuro, ed alquanto più brevi delle altre; le esterne grigie sul vessillo esterno con margine bianco nettamente distinto e l'apice grigio-bianco; la timoniera seguente, o seconda ha soltanto l'apice bianco; le remiganti hanno inferiormente nel ressillo interno larghi margini bianchi, nettamente distinti.

(387) Graucalus monotonus, Tristr.

Graucalus monotonus, Ramsay, Pr. Linn. Soc. N. s. W. VII, p. 22 (1882; - Tristr., Ibis, 1882. p. 142. - Sclat., P. Z. S. 1883 , p. 347.

Lo Sclater fa notare che nella descrizione data dal Tristram manca l'indicazione del margine cospicuamente bianco del vessillo interno delle remiganti primarie.

(388) Graucalus axillaris, SALVAD.

Graucalus axillaris, Sharpe, Gould's B. New Guin. pt. XIV. pl.4 (1883), - Nehrk., Journ. f. Orn. 1886, p. 32 (Waigeu'.

Hab. in Waigiou (Nehrkorn).

Ho esaminato gli esemplari di Waigiou menzionati dal Nehrkorn.

(389) Graucalus pusillus, Ramsay.

Graucalus salomonensis, Ramsay, Pr. Linn. Soc. N. S. W. VII, p. 22 :1882).

Graucalus dussumieri, Tristr., Ihis, 1882, p. 142.

Graucalus pusillus, Tristr., Ibis, 1882, p. 142. - Sharpe, Gould's B. New Guin. pt. XVII, pl. 4 (1884). - Grant, P. Z. S. 1887, p. 331; 1888, p. 196.

Iride gialla (Woodford). 
SP. 1086 (389 bis) Graucalus holopolius, SHARPE.

Graucalus holopolius, Sharpe, P. Z. S. 1888, p. 184。 - Grant, ibid., p. 196.

Foem. Supra grisco-caerulescens; tectricibus alarum praesertim majoribus pallidioribus quam dorso; alula spuria, tectricibus remigum primariarum remigibusque nigris exterius griseo-cinereis, secundariis ultimis pallidius intus marginatis; rectricibus nigris, mediis basin versus griseo-cinereis; 7ovis obscure cinereis, superius albo maculatis; auricularibus cinereis obscurioribus, striis scapalibus albis notatis; genis et corpore subtus 7aete griseo-caeruleis, pectoris abdominisque plumis obsolete albido marginatis; tibiis et subcaudalibus griseo-caeruleis, subalaribus palTidioribus, obsolete fasciis transversis albido-cinereis notatis; remigibus subtus fusconigricantibus, basin versus cineraceis, in pogonio interno albo marginatis; rostro, pedibus et iride nigris.

Long. tot. $0^{\mathrm{m}}, 228$; al. $0^{\mathrm{m}}, 109$; caud. $0^{\mathrm{m}}, 091$; rostri $0^{\mathrm{m}}, 019$; tarsi $0^{\mathrm{m}}, 021$.

Hab. in Papuasia - Ins. Salomonis, Guadalcanar (Woodford).

Il tipo di questa specie è una femmina, che lo Sharpe inclinava ad attribuire al Gr. pusillus, Ramisay, ma il colore nero dell'iride, che in questo è gialla, lo ha deciso a considerarlo come distinto. Aggiunge lo Sharpe che esso differisce dal Gr. pusillus per non avere la fronte e le redini nere, e per le piume auricolari sottilmente striate di bianco. A me sembra una specie dubbia e da ristudiare con maggior numero di esemplari.

(390) Graucalus sublineatus, Sclat.

Graucalus sublineatus, Ramsay, Pr. Linn. Soc. ^., S. W. VII, p. 22 (1882), - Tristr., Ibis, 1882, p. 142

(392) Edoliisoma melas (S. MüLL.).

Campephaga atra. Bernst (nec Vieill.', in Musschenbr., Dagboek, p. 145 (Sorong) (1883).

Ceblepyris atra, Bernst., op. cit., p. 147 (Sorong) (1883).

Edoliisoma melas, Sharpe, Journ. Linn. Soc., Zool. XVI, p. 434 (Norocco, Taburi) (1882). - Meyer, Zeitschr. f. ges. Orn. 1884, p. 282. - Finsch u. Meyer, op. cit. 1886, p. 18 (Montỉ Hufeisen).

Edoliisoma melan, Guillem., P. Z. S. 1883, p. 634 (Waigiou (?), Jobi).

Sarebbe desiderabile che si potesse confrontare l'esemplare (femmina) di Waigiou, menzionato dal Guillemard, potendo forse appartenere ad una specie distinta. Il Guillemard lo dice più scuro di un'altra femmina di Jobi.

(394) Edoliisoma montanum (MEYER).

Edoliisoma montanum, fiuillem., P. Z. S. 1885, p. 634 (Arfak). - Meyer, Zeitschr. f. ges. Orn. 1886, p. 18.

Hab. in Montibus Hufeisen dictis (Hunstein).

Il Meyer dice che un esemplare dei Monti Hufeisen corrisponde abbastanza con quelli degli Arfak, ma che ba le ali 10 millimetri più corte (128). Non ho potuto esaminare questo esemplare, che non è-stato acquistato dal Museo di Dresda.

(395) Edoliisoma schisticeps (G. R. GR.).

Edoliisoma schisticeps, Guillem., P. Z. S. 1885, p. 634 (Mysul). 
(999) Edoliisoma poliopse, Sharpr.

Edoliisoma pollopse, Sharpe, Gould's B. New Guin. pt. XXII, pl. 5 (1886).

Mas mari E. schisticipitis ( $\left.\mathrm{Gr}_{*}\right)$ simillimus.

Long. tot. $0^{\mathrm{m}}, 203$; al: $0^{\mathrm{m}}, 111$; caud. $0^{\mathrm{m}}, 082$; rostri culm. $0^{\mathrm{m}}, 019 ;$ tarsi $0^{\mathrm{m}}, 022$.

Foem. Cinnamomea, similis foeminae E. schisticipitis, sed mento, genis anticis et regione parotica tota schistaceis distinguenda.

Long. tot. $0^{\mathrm{m}}, 206$; al. $0^{\mathrm{m}}, 106$; caud. $0^{\mathrm{m}}, 086$; rostri $0^{\mathrm{m}}, 020$; tarsi $0^{\mathrm{m}}, 021$.

Sp. 1087 (396 bis) Edoliisoma nehrkorni, SALVAD, nov. sp.

Edoliisoma remotum, Nehrkorn (nec Sharpe), Journ. f. Orn. 1885, p. 32 (Waigeu). - Salvad: Ibis, 1886, p. 152 .

Mas. Obscure caeruleo-plumbeus, loris nigerrimis, genis et gula nigris nitentibus; alis caudaque nigris, tectricibus alarum superioribus remigibusque externe dorsi colore marginatis, remigibus intus late et abrupte canis; tectricibus alarum. inferioribus fusco-plumbeis, majoribus pallidioribus; rectricibus extimis ad apicem caerulescente-plumbeis, mediis duabus caerulescente-plumbeis, rachide et macula lata subapicali nigris exceptis; rostro pedibusque nigris.

Long. tot. circa $0^{\mathrm{m}}, 240$; al. $0^{\mathrm{m}}, 123$; caud. $0^{\mathrm{m}}, 095$; rostri $0^{\mathrm{m}}, 020$; tarsi $0^{\mathrm{m}}, 022$.

Hab. in Waigiou (Platen).

Obs. Edoliisoma E. meyeri, Salvad. simillimum, sed paullo pallidius.

Ho esaminato l'esemplare menzionato dal Nehrkorn, che generosamente lo ha donato al Museo zoologico di Torino; esso somiglia all' $E$. meyeri avendo com'esso le gote e la gola nere lucenti, ma ne differisce per essere di colore plumbeo un poco più chiaro, specialmente sulle timoniere mediane. Sebbene la differenza sia lievissima, tuttavia non dubito che esso appartenga ad una specie distinta, e che differenze più cospicue appariranno quando si troverà la femmina, giacchè, come è noto, nelle specje affini del genere Edotiisoma le femmine differiscono molto più dei maschi.

\section{Sp. 1088 (398 bis) Edoliisoma erythropygium, SHARPE.}

Edolisoma erythropygium, Sharpe, P. Z. S. 1888, p. 184.

Edolisoma erythropygon, Grant, P. Z. S. 1888, p. 196.

Mas simitis mari E. remoti, sed colore griseo obscuriore; rostro pedibusque nigris; iride saturate brunnea.

Long. tot. $0^{\mathrm{m}}, 228$; al. $0^{\mathrm{m}}, 115$; caud. $0^{\mathrm{m}}, 059$; rostri $0,{ }^{\mathrm{m}} 024$; tarsi $0^{\mathrm{m}}, 022$.

Foem. Similis foeminae $\mathbf{E}$. remoti, sed cauda omnino cinnamomea (an castanea?) et dorso imo, uropygio et supracaudalibus pallide rufis distinguenda; rostro pedibus et iride nigris.

Long. tot. $0^{\mathrm{m}}, 203$; al. $0^{\mathrm{m}}, 111$; caud. $0^{\mathrm{m}}, 076$; rostri $0^{\mathrm{m}} 021$; tarsi $0,{ }^{\mathrm{m}} 020$.

Hab. in Papuasia - Ins. Salomonis (Woodford).

Non è indicato in quale delle isole Salomone questa specie sia stata trovata. 


\section{(399) Edoliisoma obiense (SALvad.).}

Campephaga obiensis, Guillem., P. Z. S. 1885 , p. 570 (Bisa Isl., Obi Group?.

\section{(401) Edoliisoma incertum (MEYER).}

Edoliisoma incertum, Meyer, Sitzb. u. Abh. Gesellsch. Isis, 1884, Abh. I, p. 29. - Guillem., P. Z. S. 1885 , p. 635 (Jobi).

Il Meyer menziona un esemplare di Andai, aruto dal Laglaize, il quale differisce dall'esemplare tipico di Jobi pei seguenti rispetti;

1. Il colorito generale è di un azzurro (ceruleo-plumbeo?) alquanto più vivo.

2. Il becco è più lungo e più sottile.

3. Le ali e la coda sono alquanto più brevi.

4. Le cuopritrici inferiori delle ali e le ascellari sono di color nericcio e fasciate di bianco, più distintamente (lebhafter) che non nell'esemplare di Jobi.

5. Le piume del sottocoda sono dotate di strie bianco-rossicce lungo lo stelo, ed in parte di margini apicali bianchi; questi mancano nell'esemplare di Jobi.

6. La macchia apicale della timoniera esterna è più grande e di forma diversa.

7. Le timoniere mediane hanno una piccola macchia apicale, che manca nell'esemplare di Jobi.

Il Meyer conclude che forse l'esemplare di Andai appartiene ad una specie non ancora descritta.

\section{(402) Edoliisoma muelleri, SALVAD.}

Edoliosoma muelleri, Salvad, Voy. Ghall., Birds, p. 78 (1881). - Veyer, Verh. z.-h. Gesellsch. Wien, 1881, p. 773 (Aru).

\section{(405) Edoliisoma ceramense (BP.).}

Edoliisoma ceramensls, Meyer, Sitzb. u. Abh. Gesellsch. Isis, 1884, Abh. I, p. 28.

Il Meyer afferma di aver ricevuto un esemplare raccolto in Ceram dal Riedel, e che perciò il nome sopra usato deve avere la priorità.

\section{(406) Edoliisoma salomonis, Tristr.}

Edoliisorna marescottii, Ramsay (nec G. R. Gr.), Pr. Linn. Soc. N. S. W. IV, p. 71 (1879).

Edoliisoma tristrami, Ramsay, Pr. Linn. Soc. N. S. W. VII, ก. 22 (1882).

Edoliisoma salomonis, Ramsay, ibid. p: 662 (1882): - Tristr., Ibis, 1882, pp. 137, 142.

Edoliosoma marescoti, T'ristr., Ibis, 1882, p. 142.

Il Ramsay ha creduto di dover cambiare il nome di E. salomonis, Tristr. in quello di E. tristrami, per evitare la confusione possibile col Graucalus salomonensis, Ramsay, ma non credo che il cambiamento sia giustificato, trattandosi di due specie appartenenti a due generi distinti.

Il Tristram (l. c.), sulla fede del Ramsay, ha annoverato l'E. marescotti fra gli uccelli delle isole Salomone; ma il Ramsay da ultimo ha identificato il suo $E$. marescotti colla specie presente, la quale è incompiutamente conosciuta, avendo il Tristram descritto soltanto la femmina. Si noti che il Ramsay disse di aver riceruto il maschio e la femmina adulti ed anche $\mathrm{i}$ giovani. 
(408) Lalage atrovirens (G. R. Gr.).

$?$ Ceblepyris fera, Bernst. in Musschenbr., Dagboek, p. 111 (Sorong) (1883:.

Lalage atrovirens, Guillem., P. Z. S. 1885, p. 635 (Arfakl).

La localita Arfak indicata dal Guillemard è forse erronea. Questa specie fu trovata presso Sorong dal Bernstein, e forse ad essa si riferisce la Lalage fera, Bernst.

(409) Lalage tricolor (Sw.).

Lalage tricolor, Ramsay, Tab. List, p. 5 (1888).

Sp. 1089 ( $409^{\text {bis }) ~ L a l a g e ~ m o e s t a, ~ S c l a t . ~}$

Lalage moesta, Sclat., P. Z. S. 1883, pp. 51, 55, 194, 200 (Ins. Tenimber). - Sharpe, Gould's B. New Guin. pt. XV, pl. 5. - Forbes, P. Z. S. 1884, p. 432 - Meyer, Zeitschr. f. ges. Orn. I. p. 194 (1884). - Büttik., Not. Leyd. Mus, VIII, p. 63 (1886).

Supra nigra, nitore pallido virescente; fascia supra lora et uropygio albis; supracaudalibus nigris, albo terminatis; loris nigris; corpore subtus omnino albo; tectricibus alarum mediis et majoribus, remigibus secundariis exterius, apicem versus late albo marginatis; subalaribus et remigibus intus basin versus albis; cauda nigra, rectricibus duabus externis late albo-terminatis; rostro et pedibus nigris. Foem. Supra nigra, sed minus nitida, et fascia uropygiali albo-cineracea diversa.

Long. tot. $0^{\mathrm{m}}, 177$; al. $0^{\mathrm{m}}, 097$; caud. $0^{\mathrm{m}}, 076$; rostri culm. $0^{\mathrm{m}}, 012$; tarsi $0^{\mathrm{m}}, 022$.

$H a b$. in Ins. Tenimberensibus (Forbes, Riedel).

Ho descritto un esemplare raccolto dal Riedel ed inviato dal Meyer al Museo di Torino.

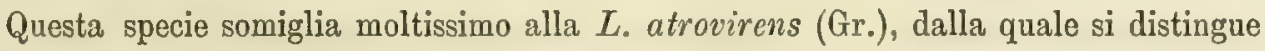
facilmente per avere la fascia bianca sopra le redini, che dalle narici si estende fino all'occhio, senza formare un vero sopracciglio.

(410) Lalage karu (LESS.).

Lalage karu, Salvad., Voy. Chall, Birds, p. 69 (1881). - Finsch, Vög. d. Südsee, p. 12 (Nova Britannia) (1884.

Lalage leucomelaena, Mamsay, Tab. List, p. 5 (1888).

(411) Lalage aurea (TEMM.).

Lalage aurea, Pleske, Bull. Acad. Petersb. XXIX, p. 529 (Ternate) (1884). - Guillem., P. Z. S. 1886 p. 570 (Ternate),

(412) Symmorphus affinis, TrISTR.

Symmorphus affinis, Ramsay, Pr. Linn. Soc. N. S. W. VII, p. 23 (1881). - Tristr., Ibis, 1882, pp. $137,142$.

(414) Artamus leucogaster (VaLenc.).

Artamus leucogaster, Meyer, Verh. z.-b. Ges. Wien, 1881, pp. 766 (Sumba), 769 (Wetter). -Sharpe, Journ. Linn. Soc., Zool. XVI, p. 434 (1882). - Finsch, Vög. d. Südsee, p. 27 (1884). - - Pleske, 
Bull. Acad. Petersb. XXIX, p. 530 (Ternate) (1884). - Nehrk., Journ. f. Orn. 1885, p. 32 (Waigeu).

Guillem., P. Z. S. 1885, p. 635 (Waigiou). - Ramsay, Tab. List, p. 3 (1888).

Artamus leucopygialis, Meyer, Zeitschr. f. ges. Orn. I, 1886, pp. 18, 35.

Il Meyer vorrebbe che gli esemplari della Nuova Guinea meridionale-orientale. come quelli dell'Australia, alquanto più piccoli di quelli delle altre località, dovessero essere considerati come specificamente distinti sotto il nome di A. leucopygialis, Gould. Egli dà le seguenti dimensioni per gli esemplari della Nuova Guinea meridionaleorientale : ala $0^{\mathrm{m}}, 128-0^{\mathrm{m}}, 130$; coda $0^{\mathrm{m}}, 058-0^{\mathrm{m}}, 061$; becco $0^{\mathrm{m}}, 0155-0^{\mathrm{m}}, 017$; queste dimensioni egli le confronta con quelle degli esemplari di Sumatra, Borneo, Isole Filippine, Celebes, Halmahera, Ternate, Batanta, Wetter e Sumba, i quali sono costantemente alquanto più grandi; inoltre egli trova un' altra differenza nella minore estensione del nero della gola; con tutto ciò a me non sembra possibile di separare specificamente quegli esemplari.

\section{Sp. 1090 (414 bis) Artamus musschenbroeki, Meyre.}

Artamus leucogaster, Sclat. (nec Val.), P. Z. S. 1883, pp. 51, 195, 200 (Larat, Kirimoen). - Forbes, P. Z. S. 1884, pp. 427, 432, n. 27. - Id., Natural. Wander. p. 361 (1885). - Büttik., Not. Leyd. Mus. VIII, p. 63 (1886).

Artamus Musschenbroeki, Meyer, Sitzb. u. Abh. Gesellsch. Isis, 1884, Abh. 1, p. 30 (1884) (Tenimber et Timur-laut). - Salvad, Ibis, 1884, p. 355. - Id., P. Z. S. 1884, pp. 577, 578. - Meyer, Zeitschr. f. ges. Orn. III, p. 20 (1886).

Artamus A. lencogastri (Valenc.) simillimus, sed obscurior, nigricans, cauda fasciola terminali alba ornata et rostro longiore diversus.

Long. tot. $0^{\mathrm{m}}, 180$; al. $0^{\mathrm{m}}, 130-0^{\mathrm{m}}, 140$; caud. $0^{\mathrm{m}}, 065-0^{\mathrm{m}}, 073$; rostri $0^{\mathrm{m}}, 020-$ $0^{\mathrm{m}}, 021 ; \operatorname{tarsi} 0^{\mathrm{m}}, 017-0^{\mathrm{m}}, 018$.

Hab. in Papuasia - Ins. Tenimberensibus (Forbes, Riedel).

Io ho esaminato sei degli esemplari tipici del Meyer, due dei quali si trovano ora nel Museo di Torino; essi mi sembrano sufficientemente distinti dall' $A$. leucogaster (Valenc.) pei caratteri sopraindicati.

(415) Artamus maximus, MEYER.

Artamus maximus, Guillem., P. Z. S. 1886, p. 635 (Arfak!.

Sp. 1091 (417 bis) Dicruropsis guillemardi, SALVAD. nova sp.

Dicruropsis sp., Guillem., P. Z. S. 1885, p. 571 (Bisa Isl., Obi Group).

Iride bruna, becco e piedi neri (Guillemard).

liung. tot. $0^{\mathrm{m}}, 318$; al. $0^{\mathrm{m}}, 160$; coda $0^{\mathrm{m}}, 145$; becco dall'angolo della bocca $0^{\mathrm{m}}, 035$.

Il Guillemard menziona un esemplare di Bisa, nel gruppo di Obi, che egli non riesce ad identificare. Egli afferma che è diverso dalla $D$. atrocaenulea per le macchie pettorali più grandi, per le dimensioni minori e per le piume allungate metalliche sui lati del collo, ed aggiunge che la sola specie di Obi finora conosciuta è la $D$. pectoralis Wall., e che in questa le macchie pettorali sono più grandi, più cospicue e volgenti più al verde, 'e le lunghe piume dei lati del collo più grandi e più lunghe. 
Sembrandomi difficile che nel gruppo di Obi vivano due specie affini, io penso che gli esemplari di quelle isole appartengano ad un'unica specie, alla quale provvisoriamente ho dato il nome di $D$. guillemardi. Il Guillemard non ha potuto inviarmi l'esemplare di Bisa, avendo ceduto la sua collezione ad uno dei Rotschild.

\section{(418) Dicruropsis laemosticta (ScLaT.)}

Dicrurus laemostictus, Finsch, Vög. d. Südsee, p. 13 (Nova Britannia) (1884).

\section{Sp. 1092 (418 $\left.{ }^{\text {bis }}\right)$ Dicruropsis propinqua (TRISTR.).}

Ghibia propinqua, Tristr., Ibis, 1889, p. 556.

Dicruropsis D. Inemostictae (Sclat.) propinqua, sed differt maculis nitentibus colli antici imi et pectoris summi valde angustioribus et elongatis, neque, sicut in D. carbonaria, rotundatis. Statura sicut in D. Inemosticta.

Hab. in Ins. 'd'Entrecasteaux (Thomson).

Il Tristram avverte che $\mathrm{i}$ caratteri distintivi di questa specie si apprezzano meglio col confronto di quello che si possano valutare dalla descrizione.

\section{(419) Dicruropsis bracteata (GodLD).}

Dicruropsis bracteatus, Sclat., P. Z. S. 1883, pp. 51, 200. - Salvad., Ibis, 1884, p. 355. - Neyer, Zeitschr. f. ges. Orn. I, p. 194 (1884). - Forbes, P. Z. S. 1884, p. 432, n. 28.

Dicrurus bracteatus, Finsch, Vög. d. Südsee, p. 35 (1884).

Chibia bracteata, Ramsay, Tah. Lisi, p. 5 (1888).

Dicruropsis bracteata, Salvad., Ibis, 1884, p. 355. - Id., P. Z. S. 1884, p. 377.

Hab. in Ins. Tenimberensibus, Larat (Forbes).

Credo che gli esemplari delle Isole Tenimber dotranno essere nuovamente confrontati con quelli della Nuova Olanda e della Nuova Guinea meridionale-orientale.

(420) Dicruropsis megalornis (G. R. GR.).

Dicruropsis megalornis, Salvad., Voy. Chall., Birds, p. 69 (1881). - Meyer, Verh. z.-b. Ges. 1881 p. 734 (Watubella, o Matabella).

(421) Dicruropsis atrocaerulea (G. R. GR.).

Dicruropsis atrocaerulea, Guillem., P. Z. S. 1885, p. 570 (Batchian).

(422) Dicruropsis carbonaria (S: MüLL.).

Dicruropsis assimilis, Salvad., Voy. Chall., Birds, p. 79 (1881).

Chibia carbonaria, Sharpe, Journ. Linn. Soc., Zool. XVI, p. 434 (Taburi) (1882).

Dicruropsis carbonaria, Nehrk., Journ. f. Orn. 1885, p. 32 (Waigeu).

Dicruropsis carbonarius, Meyer, Zeitschr. f. ges. Oru. I, 1886, p. 35 (Kafu).

(1000) Dicruropsis? longirostris (RAMSAT).

Dicrurus longirostris, Ramsay, Pr. Linn. Soc. N. S. W. VII, p. 668 (1882).

(425) Dicranostreptus megarhynchus (Q. et G.).

Dicrurus megarhynchus, Tristr., Ibis, 1882, p. 142. - Ramsay, Pr. Linn. Soc. N. S. W. VII, p. 43 (1882). - Finsch, Vög. d. Südsee, p. 13 (Now-Irland) (1884). 
Pare che questa specie non si trovi nelle isole Salomone, ma soltanto nella Nuova Irlanda.

(426) Chaetorhynchus papuensis, MEYRR.

Ghaetorhynchus papuana (sic), Ramsay, Pr. Linn. Soc. N. S. W. X, p. 244 (Mount Astrolabe) (1885). Ghaetorhynchus papuensis, Finsch u. Meyer, Zeitschr. f, ges. Orn. 1886, p. 20 (Monti Hufeisen).

\section{(427) Gracticus cassicus (Bodd.).}

Cracticus cassicus, Salvad, Voy. Chall., Birds, p. 80 (1881\% - Gad., Cat. B. VIII, p. 97 (1883). Finsch, Vög. d. Sïdsee, p. 27 (1884). - Meyer, Zeitschr, f. ges. Orn. I, p. 283, Taf. XVIII, f. I (uovo) (1884). - Nehrk., Journ. f. Orn. 1885, p. 32 (Waigeu) - Guillem., P. Z. S. 1885, p. 635.

Il Meyer descrive anche il nido.

(428) Gracticus mentalis, SaLVAD. et D'ALB.

Cracticus mentalis, Gad,, Cat. B. VIII, p. 102 (1883). - Finsch, Vög. ả. Südsee, p. 27 (Port Moresby) (1884).

\section{Sp. 1093 (428 bis) Gracticus louisiadensis, Tristr.}

Gracticus louisiadensis, Tristr., Ibis, 1889, p. 555.

Cracticus nigerrimus; abdomine imo et subcaudalibus albis; supracaudalibus albo terminatis; subalaribus albis; humeralibus apice albis, remigibus secundariis quinto et sexto albis, nigro terminatis; septimo in pogonio externo albo, nigro terminato; rectricibus tribus externis macula alba in pogonio interno prope apicem praeditis; rostro margaritaceo, pedibus nigris.

Long. tot. 12.1 poll. angl. $\left(=0^{\mathrm{m}}, 307\right)$; alae $6.25\left(=0^{\mathrm{m}}, 158\right)$; caudae 6 . $\left(=0^{\mathrm{m}}, 154\right)$; tarsi $1.1\left(=0^{\mathrm{m}}, 028\right)$; rostri $1.7\left(0^{\mathrm{m}}, 043\right)$.

Hab. in insula Sud Est dicta (Thomson).

Questa specie, come fa notare il Tristram, differisce pel suo modo di colorazione da tutte quelle conosciute, tuttaria pel colorito generale nero sembra che si avricini al $C r$. quoyi (Less.).

(429) Gracticus quoyi (LESS.).

Gracticus quoyi, Gadow, Cat. B. VIII, p. 94 (1883). - Finsch, Vög. d. Südsee, p. 36 (1884). Meyer, Zeitschr. f. ges. Orn. I, p. 283, Taf. XVIII, f. $2-4$ (uova) (1884). - Nehrk., Journ. f. Orn. 1885, p. 32 (Waigeu). - Guillem., P. Z. S. 1885, p. 636. - Ramsay, Tab. List, p. 4 (1888).

11 Meyer oltre alle uova descrive anche $\mathrm{i}$ nidi.

\section{(430) Grallina bruijni, Salvad.}

Grallina bruijni, Sharpe, Gould's B. New Guin. pt. XIV, pl. 5 (1883). - Meyer, Zeitschr. f. ges. Orn. 11l, p. 20 (1886).

Mas. Foeminae similis, sed gula et pectore toto nigris; capite nigro, capitis et colli lateribus maculis duabus albis, alia ad occipitis latera, altera juxta colli latera excurrente, notatis.

Io descrissi soltanto la femmina di questa specie, lo Sharpe ha descritto il maschio e figurato ambedue $\mathrm{i}$ sessi.

[222] 
(431) Rhectes uropygialis, G. R. GR.

Rectes uropygialis, Guillem.,P.Z.S. 1885, p. 636 (Mysol, Salwatti).-Sharpe, Ibis, 1888, pp.435, 437. Rectes tibialis, Sharpe, J. c.

Il Guillemard fa notare che le femmine hanno la testa di un nero meno puro dei maschi, la fronte e le redini di color bruno, il color cannella del dorso e delle parti inferiori più pallido. Sarebbe desiderabile che gli esemplari menzionati dal Guillemard fossero nuovamente esaminati.

(432) Rhectes aruensis, SHARPE.

Rectes aruensis, Salvad., Voy. Chall., Birds, p. 79 (1881) - Guillem., P. Z. S. 1885, p. 636 (Aru). Sharpe, Ibis, 1888, pp. 435, 437. - Id., Gould's B. New Guin. pt. XXV, pl. 5 (1888).

Rhectes analogus, Meyer, Zeitschr. f.ges. Orn. I, p. 284, Taf. XIV, f. 2, XV, f. 2 (1884) (Aru).

Rectes analogus, Sharpe, This, 1888, p. 427.

Credo che abbia ragione lo Sharpe il quale afferma che il $R$. analogus, Meyer è fondato sopra esemplari giovani del $R$. aruensis. Il Meyer nel separare il suo $R$. analogus è stato influenzato dai rapporti fra il $R$. analogus ed il $R$. aruensis analoghi a quelli che passano fra il $R$. cirrhocephalus ed il $R$. dichrous, rispetto al colorito, ma non ha considerato che l'analogia cessa quando si consideri la loro distribuzione geografica, giacchè mentre il $R$. cirrhocephatus ed il $R$. dichrous, si trovano insieme nella parte settentrionale-occidentale della Nuova Guinea, più non si trovano insieme lungo il fiume Fly e nella parte meridionale-orientale, laddove gli esemplari riferibili al $R$. analogus ed al $R$. aruensis, si trovano insieme nelle isole Aru, ove il Beccari ha ucciso contemporaneamente una femmina, che sarebbe riferibile al primo, e due maschi riferibili al secondo.

Lo Sharpe ha ommesso di citare le figure date dal Meyer del suo $R$. analogus.

\section{Sp. 1094 (432 bis) Rhectes meridionalis, SHARpE (?).}

$?$ Rectes dichroa, Salvad, (nec Bp.), Ann. Mus, Civ. Gen. IX, p. 30 (1876) (Naiabui).

Rhectes dichrous, partim (?), Salvad, Orn. Pap. e Nol. II, Pp. 195, 196 (1881) (Specimina t, u, v, ex Naiabui?). - ? Sharpe, Journ, Linn. Soc., Zool. XVI, p. 435 (Morocco, China, Straits) (1882).

- Meyer, Zeitschr. f. ges. Orn. III, p. 20 (1886).

Rectes meridionalis, Sharpe, Ibis, 1888, p. 437.

Rhectes similis R. uropygiali, sed scupularibus dorso concoloribus, nec nigris, et uropygio (!) tantum nigro distinguendus (Sharpe).

Long. tot. $0^{\mathrm{m}}, 254$; al. $0^{\mathrm{m}}, 132$; caud. $0^{\mathrm{m}}, 109$.

Hab. in Papuasia - Nova Guinea meridionali-orientali, Montibus Astrolabii (Hunstein).

Lo Sharpe crede che a questa specie siano da riferire tre esemplari di Naiabui, raccolti dal D'Albertis, dei quali io giả arvertii come due differissero dai tipici esemplari del $R$. dichrous per avere qualcuna delle piume più profonde del sopraccoda nere, o variegate di nero, per cui (aggiungeva) pare quasi che negli escmplari della Nuova Guinea meridionule-orientale cominci un principio di differenziazione, per la quale si avvicinano al $R$. uropygiatis ed al $R$. aruensis. Ad onta di tutto ciò io debbo confermare che $\mathrm{i}$ tre esemplari di Naiabui non si possono separare dal $R$. di- 
chrous, e credo che il $R$. meridionalis abbisogni di altri esemplari per essere ammesso come distinto. Lo Sharpe manifesta inoltre il dubbio che questa sua specie sia fondata sopra i maschi della $R$. brunneiceps, Salvad., la quale cosa è assolutamente inammissibile, giacchè $\mathbf{i}$ tịi della $R$. brunneiceps erano maschi adulti, non averano punto la testa nera e sono ben distinti pel colore della coda (rufo-brunnea) e per altri caratteri.

\section{(433) Rhectes dichrous, Bp.}

Rhectes dichrous, Salvad., Orn. Pap. e Mol. II, p. 197 (partim) (1881). - ? Sharpe, Journ. Linn. Soc., Zool. XVI, p. 435 (Morocco, China Straits) (1886). - Meyer, Sitzb. u. Abh. Gesellsch. Isis, 1884, Abh. I, pp. 32, 33, 34. - Guillem., P. Z. S. 1885, p. 636 (న. Coast of Papua, long. 139॰ E, Bruijn).

Rhectes dichrous, Sharpe, Ibis, 1888, pp. 435̄, 436, 437.

È singolare che lo Sharpe a malincuore ammetta che il $R$. dichrous colla testa nera non è l'adulto del $R$. cirrhocephalus, colla testa grigia chiara, ma una specie affatto distinta, come io credo di aver provato, dopo aver mostrato che i giovani da nido del $R$. dichrous banno la testa assolutamente nera come gli adulti. Anche lo Sharpe menziona un giovane nidiaceo del Museo Britannico che ha la testa e la gola nere come gli adulti, ed io non saprei quale altra prova maggiore si possa dare per convincere lo Sharpe! Tuttavia gliene darò ancora una: egli ammette che il $\boldsymbol{R}$. dichrous si trovi anche nella Nuova Guinea meridionale-orientale, ove, per quanto io sappia, nessuno ha mai trovato esemplari riferibili al $R$. cirrhocephalus.

\section{(434) Rhectes decipiens, SALVAD.}

Rectes decipiens, part., Salvad., Ann. Mus. Civ. Gen. p. 473 (1878) (excl. specim.'ex Rubi). Sharpe, Ibis, 1888, pp. 436, 437.

Rhectes decipiens, part., Salvad., op. cit. XV, p. 42, n. 8 (1879). - Id., Orn. Pap.e Mol. II, p. 197 (partim) (1881). - Meyer, Sitzb. u. Abh. Gesellsch. Isis, 1884, Abh. I, pp. 33, 34. - Id., Zeitschr. f. ges. Orn. I, p. 285, Taf. XV, f. 3 (1884).

Rufo-cinnamomeus, subtus valde pallidior; capite cristato toto et gutture fuscocinereis, fere nigricantibus; alis et cauda fusco-nigricantibus, remigibus intus pallidioribus, minime rufescentibus; rostro, pedibus et iride fuscis.

Long. tot. $0^{\mathrm{m}}, 280-0^{\mathrm{m}}, 248$; al. $0^{\mathrm{m}}, 130-0^{\mathrm{m}}, 120$; caud. $0^{\mathrm{m}}, 122-0^{\mathrm{m}}, 110$; rostri $0^{\mathrm{m}}, 028-0^{\mathrm{m}}, 026 ;$ tarsi $0^{\mathrm{m}}, 033-0^{\mathrm{m}}, 032$.

Hab. in Papuasia - Nova Guinea, prope Nappan, Waweji, Inviorage (Meyer); Mambriok, ins. Amberpon, Rhoon (Laglaize).

Il Meyer vorrebbe che questa specie fosse confinata al Nord di Rubi (ove si trova il suo $R$. rubiensis), sulla costa occidentale della Baia del Geelwink, nella regione ove esistono le località Nappan, Waweji, Inviorage, Mambriok, e nelle vicine isole di Amberpon e Rhoon. Egli (dopo l'esclusione degli esemplari di Rubi) ha dovuto correggere la mia descrizione del $R$. decipiens, Salvad., dicendo capite cristato toto, gutture, alis et cauda nigris, mentre invece più esattamente doveva dire griseo-nigricantibus. 


\section{Sp. 1095 ( $434^{\text {bis }}$ ) Rhectes rublensis, MeYer.}

Rhectes cirrhocephalus, part., Mleyer, Sitzb. Akad. Wien, XLIX, p. 207 (1874) (Specimina ex Rubi). Rhectes decipiens, part., Salvad., Ann. Mus. Civ. Gen. XII, p. 473 (1878)(Specim. ex Rubi et Warbusi); XV, p. 42, n. 8 (1879). - Id., Orn. Pap. e Mol. II. p. 197 (partim) (1881).

Rhectes rubiensis, Meyer, Sitzb. u. Ahh. Gesellsch. Isis, 1884, Abh. I, pp. 32, 34 (Rubi). - Id., Zeitschr. f. ges, Orn. I, p. 285, Taf. XIV, f. 1, XV, f. 1 (1884).

Rectes rubiensis, Sharpe, Ibis, 1888, pp. 436, 437.

Pallide rufo-cinnamomeus, subtus brunnescenti-ochraceus; capite cristato toto et guture fusco-cinereis, paullum brunneo tictis; alis et cauda fusco-nigricantibus; remigibus exterius brunnescenti-griseo, intus basin versus isabellino marginatis; subalaribus unicoloribus pallide rufis; rostro fusco (Meyer).

Foem. In toto paullo pallidior.

Long. tot. $0^{\mathrm{m}}, 260-0^{\mathrm{m}}, 255$; al. $0^{\mathrm{m}}, 125-0^{\mathrm{m}}, 120$; caud. $0^{\mathrm{m}}, 120-0^{\mathrm{m}}, 113$; rostri culm. $0^{\mathrm{m}}, 028-0^{\mathrm{m}}, 027$; tarsi $0^{\mathrm{m}}, 032-0^{\mathrm{m}}, 031$.

Hab. in Papuasia - Nova Guinea prope Rubi (Meyer), Warbusi (Beccari).

Il Meyer ha creduto di dover separare specificatamente gli esemplari di Rubi, nella parte più meridionale della Baia del Geelwink, dal mio $R$. decipiens, che sarebbe confinato più al Nord sulla costa occidentale della Baia del Geelwink, in alcune isole vicine ed anche sulla costa occidentale della Nuova Guinea al sud del golfo del Mac Cluer. Lo Sharpe, il quale non sembra disposto ad ammettere nè il $R$. decipiens, nè il $R$. rubiensis, perchè forme intermedie al $R$. dichrous ed al $R$. cirrhocephalus, è caduto in errore, perchè non ha considerato la diversa distribuzione geografica.

Il Ieyer ha affermato che il suo $R$. rubiensis $\grave{e}$ affine specialmente al $R$. brunneiceps, D'Alb. et Salvad. del fiume Fly, ma questa cosa non è esatta, giacchè il $R$. brunneiceps è specie affatto distinta anche pel colore bruno-rossigno della coda. Il $R$. mbiensis è una forma del $R$. decipiens, dal quale si distingue principalmente pel colorito della testa grigio più chiaro e tinto leggermente di bruno e pel colore castagno del dorso più chiaro.

\section{(435) Rhectes cirrhocephalus (LESS.).}

Rhectes cirrhocephalus, Meyer, Sitzb. u. Abh. Gesellsch. Isis, 1884, Abb. 1, pp. 32, 33, 34. Rectes cirrhocephalus, Sharpe, Ibis, 1888, pp. 435, 436, 437.

Non potrà mai ripetersi abbastanza che questa specie non è fondata sui giovani del $\boldsymbol{R}$. dichrous, come vorrebbe ancora lo Sharpe, e che invece essa n'è affatto distinta per caratteri e per diversa distribuzione geografica.

Gli esemplari intermedii al $R$. dichrous ed al $R$. cirrhocephalus ( $R$. decipiens e $R$. rubiensis) non si trovano nella regioue abitata da quelle due specie (Dorei, Andai, Mansinam e Monti Arfak), ma più al sud sulla costa occidentale della Baia del Geelwink e presso Rubi.

\section{(436) Rhectes brunneiceps, Salvad.}

? Rectes brunneiceps, Sharpe, Ibis, 1888, pp. 436, 437 (Astrolabe Mountains).

Lo Sharpe riferisce a questa specie un esemplare dei Monti dell'Astrolabio, ma 
io credo che egli s'inganni, giacchè mi pare difficile che il $R$. brunneiceps si estenda dalla regione del fiume Fly fino ai Monti dell'Astrolabio. Non bisogna dimenticare che il R. brunneiceps, $\mathrm{i}$ tipi del quale sono tutti indicati come maschi, è caratterizzato dal colore grigio-bruno-rossigno della testa e del collo, e dalla coda di color rossignobruno cogli steli delle timoniere inferiormente chiari, come nel $R$. ferrugineus.

\section{(437) Rhectes cerviniventris, Gray.}

Rectes cerviniventris, Sharpe, Gould's B. New. Guin. pt. XX, pl. 11 (1885). - Nebrk., Journ. f. Orn. 1885, p. 33 (Waigeu). - Guillem., P. Z. S. 1885, p. 636.

(438) Rhectes jobiensis, Merer.

Rhectes jobiensis, Guillem., P. Z. S. 1885, p. 637.

(439) Rhectes cristatus, SALVAD.

Pseudorectes cristatus, Sharpe, Ibis, 1888, p. 436.

(440) Rhectes ferrugineus (S. MüLL.).

Pseudorectes ferrugineus, Sharpe, Journ. Linn. Soc., Zool, XVI, p. 435 (Morocco, China Strails) (1882). - Meyer, Zeitschr. f. ges. Orn. IlI, p. 20 (1886) (Monti dell'Astrolabio).

Rhectes ferrugineus, Ramsay, Pr. Linn. Soc. N. S. W. VIII, p. 26 (uovo) (1883). - Meyer, Sitzb. u. Abh. Gesellsch. Isis, 1884. Abh. I, p. 31 (partim). - 1d., Zeitschr. f. ges. Orn. I, p. 285 (1884) (Aru). - Guillem., P. Z. S. 1885, p. 637.

Rhectes sp., Meyer, Zeitschr. f. ges. Orn. I, p. 285, Taf. XVII, f. 5 (uovo) (1884).

Pare che il Meyer riferisca a questa specie il mio $R$. holerythrus, del quale menziona esemplari di Jobi e tre indicati dubitativamente di Misori, o di Jobi. Egli mi ha inviato uno di questi esemplari d'incerta località; esso somiglia agli esemplari ordinari del $R$. ferrngineus, ma ha dimensioni alquanto maggiori (lunghezza dell'ala $0^{\mathrm{m}}, 147$ ), e non ha nulla che fare col $R$. holerythrus.

Il Mejer, ricordando quanto io aveva già fatto notare relativamente agli esemplari delle Isole Aru, cioè che essi sono più piccoli di quelli della Nuova Guinea, accenna alla convenienza di separare quelli specificamente, tanto più che differiscono anche pel colore più vivo delle parti inferiori.

(441) Rhectes holerythrus, Salvad.

Rectes ferrugineus, part., Neyer, Sitzb. u. Abh. Gesellsch. Isis, 1884, Abh. I, p. 31 (?)(specimina ex Jobi et ex Mysori?). - Id., Zeitsehr. f. ges. Orn. I, p. 285 (1884).

(442) Rhectes leucorhynchus, Gray.

Rectes leucorhynchus, Sharpe, Gould's B. New Guin. pt. XX, pl. 10 (1885). - Nehrk., Journ. I. Orn. 1885, p. 33 (uovo, Waigeu).

Rhectes leucorhynchus, Guillem., P. Z. S. 1885, p. 637 (Waigiou).

\section{(443) Rhectes nigrescens (SchleG.).}

Melanorectes nigrescens, Meyer, Zeitschr. f. ges. Orn. III, p. 21 (1886).

Il Meyer menziona un esemplarre dei Monti Hufeisen nella Nuova Guinea meridionale-orientale e lo dice alquanto differente da quelli dei Monti Arfak per una tinta 
nera alquanto più azzurrognola, per le parti inferiori meno bruniccie, e per la coda inferiormente non bruniccia, inoltre le piume del pileo sarebbero più lunghe.

Il Meyer non ha potuto inviarmi detto esemplare, che è gia montato nel Museo di Dresda.

(444) Colluricincla brunnea, GodLd.

Colluriocincla brunnea, Ramsay, Tab. List, p. 5 (1888).

(447) Colluricincla megarhyncha (Q. et $G_{0}$ ).

Colluricincla megarhyncha, Salvad., Voy. Chall., Birds, p. 79 (1881). - Guillem., P. Z. S. 1885, p. 637 (Batanta).

$?$ Myiolestes grandis, Bernst, in Musschenbr., Daghoek, p. 113 (Sorong) (1883).

Colluricincla rufigaster, Meyer (nec Gould), Zeitschr. f. ges, Orn. 1884, p. 286 (Aru).

Il Mejer vorrebbe che gli esemplari delle Isole Aru non appartenessero alla C. megarhyncha, cui io li ho riferiti, ma alla $C$. rufigaster, Gould; io non posso persuadermi della esattezza di quella opinione, giacchè avendo anche io confrontato numerosi esemplari di quelle isole con altri della specie australiana, ho trovato che sono diversi e che piuttosto sono da riferire al $C$. megarhyncha.

\section{(1101) Colluricincla rufigaster, Gould.}

Colluricincla rufigaster, Ramsay, Pr. Linn. Soc. N. S. W. VIII, p. 28 (1883) (uova). - Finsch u. Meyer, Zeitschr. f. gez, Orn. 1886, p. 21 (Monti Hufeisen).

Pinarolestes parvulus (Gould). - Finsch, Vög. d. Südsee, p. 34 (1884).

$?$ Colluriocincla parvissima, Ramsay, Tab. List, p. 5 (1888).

Il Finsch attribuisce gli esemplari di Port Moresby alla $C$. parvula, Gould, cui riferisce il Pinarolestes rufigaster, Sharpe, Cat. B. III, p. 296.

(448) Colluricincla affinis, G. R. Gr.

Colluricincla affinis, Nebrk., Journ. f. Orn. 1885, p. 33 (Waigeu) - Guillem., P. Z. S. 1885, p. 637 (Waigiou).

Colluricincla megarhyncha, Guillem. (nec Q. et G.?), P. Z. S. 1885, p. 571 (Batchian!). - Salvad. Ibis, 1886, p. 154 .

Come ho già fatto notare (l. c.), è molto probabile che il supposto esemplare della $C$. megarhyncha creduto di Batchian sia invece un esemplare della $C$. affinis di Waigiou.

\section{(449) Pachycephala astrolabil Bp.}

Pachycephala orioloides, Ramsay, Pr. Linn. Soc. N. S. W. VIl, p. 25 (1882) (Isole Salomone).

Pachycephala astrolabi, Tristr., Ibis, 1882, pp. 137, 142. - Gadow, Cat. B. VIII, p. 200 (partim) (1883). - Grant, P. Z. S. 1888, p. 196 (Aola).

\section{Sp. 1096 (449 bis) Pachycephala fusco-flava, Sclat.}

Pachycephala, Sp. inc. O, Sclat., P. Z. S. 1883, p. 51.

Pachycephala fusco-flava, Sclat., P. Z. S. 1883, pp. 195, 198, 200, pl. XXVIII (屯 juv.). - Forbes, P. Z. S. 1883 , p. 589, pl. LIII (ठ); 1884, p. 432. - Meyer, Sitzb. u. Abb. Gesellsch. Isis, 1884, Abb. I, p. 35 (ङ). - Id., Zeitschr. f. ges. Orn. I, p. 194 (1884). - Forhes, Natural. Wander. p. 589, pl. 53 (1885). - Sharpe, Gould's B. New Guin. pt. XIX, pl. 10 (1885), - Büttik., Not. Leyd. Mus. VIII, p. 64 (1886). 
Mas. Supra viridi-olivaceus, uropygio flavicante, capite, cervice et torque pectorali, cum capite conjuncto, nigris; supracaudalibus nigris, viridi-olivaceo late marginatis; torque cervicali obsolete flavicante; gula et corpore subtus laete flavis; mento albescenti; alis fuscis, viridi-olivaceo marginatis, remigibus majoribus exterius griseo limbatis; cauda fusco-grisea, olivaceo limbata; rostro nigro; pedibus plumbeis.

Foem. Supra olivacea; subtus flavido-rufescens; auricularibus fusco-brunnescentibus; genis flavo tinctis; alis fuscis, olivaceo tinctis; cauda supra olivacea, subtus fusco-grisea.

Juv. Foeminae similis, sed alis exterius rufo-brunneo tinctis; rostro pallido. Long. tot. $0^{\mathrm{m}}, 185$; al. $0^{\mathrm{m}}, 105$; caud. $0^{\mathrm{m}}, 078$; rostri $0^{\mathrm{m}}, 021$; tarsi $0^{\mathrm{m}}, 029$.

Hab. In Papuasia - Ins. Tenimberensibus (Forbes, Riedel).

Questa specie appartiene al gruppo di quelle colla gola gialla, e sembra somigliante alla $P$. astrolabii, dalla qualo differisce pel collare cervicale giallo appena indicato. Lo Sharpe dice che essa somiglia molto alla $P$. torquata, Layard, di Taviuni (Isole Fiji), dalla quale differirebbe per essere più grande e di colorito più chiaro superiormente ed inferiormente.

Il nome specifico fusco-flava è poco adatto a questa specie; esso fu dato al maschio giovane, che servi di tipo della specie.

Io ho esaminato un esemplare giovane, raccolto dal Riedel, ed inviato al Museo di Torino dal Meyer.

\section{(450) Pachycephala christophori, Tristr.}

Pachycephala astrolabi, part., Gadow, Cat. B. Brit. Mus. VIII, p. 200 (1883).

Pachycephala chrystophori, Ramsay, Pr. Linn. S. N. W. VII, p. 25 (1882) (Isole Salomone).Tristr., Ibis, 1882, pp. 137, 142. - Salvad., Ibis, 1884, p. 323. - Tristr., Ibis, 1884, p. 398. Sharpe, Gould's B. New Guin. pt. XXIII, pl. 11 (1887).

\section{(451) Pachycephala mentalis, WaLt.}

Pachycephala mentalis, Salvad., Voy. Chall., Birds, p. 60 (1881). - Gad., Cat. B. VIII, p. 189 (1883). - Pleske, Bull. Acad. Petersb. XXIX, p. 530 (Ternate) (1884). — Guillem., P. Z. S. 1885, p. 371 (Ternate, Batchian).

Il Gadow annovera Timor tra le localita abitate da questa specie, la qual cosa certamente non è esatta, e probabilmente il giovane esemplare di Timor, che egli attribuisce alla $P$. mentalis, spetta invece alla $P$. calliope, che è appunto di Timor.

\section{(452) Pachycephala macrorhyncha, Strickl.}

Pachycephala macrorhyncha, Blas. u. Nerk., Verh. \%-b.Gesellsch. Wien,1882, p. 423 (Amboina). - Sharpe, Rep. Alert, p. 15 (1884). - Salvad., Ibis, 1884, p. 323.

Pachycephala melanura, part., Gad., Cut. B. VllI, p. 185 (1883).

È probabile che gli esemplari delle Isole Sulla debbano essere riferiti ad una specie distinta ( $P$. clio, Wall.). 
(453) Pachycephala obiensis, Salvad.

Pachycephala melanura, part., Gad., Cat. A. VIII, p. 185 (1883).

Pachycephala obiensis, Sharpe, Report Alert, p. 15 (1884). - Salvad., Ibis, 1884, p. 323. - Guillem., P. Z. S. 1885 , p. 371 .

\section{(454) Pachycephala melanura, GodLd.}

Pachycephala melanura, part., Gad., Cat. B. VIII, p. 185 (1883). - Sharpe, Report Alert, p. 15 (1884). - Salrad., Ibis, 1884, p. 323. - Ramsay, Tab. List, p. 5 (1888).

(455) Pachycephala collaris, RAMSAT.

Pachycephala collaris, Gad., Cat. B. VIIl, p. 197 (1883). - Sharpe, Gould's B. New Guin. pt, XXI, pl. 10 (1886).

\section{Sp. 1097 ( $455^{\text {bis }}$ Pachycephala sharpei, MErer.}

Pachycephala Sharpei, Neyer, Sitzb. u. Abh. Gesellsch. Isis, 1884, Abh. I, p. 36 (Ins. Babbar) (Tipo esaminato).

Capite nigro; torque pectorali nigro, plumarum scapis albis, torque muchali Aavo; notaeo reliquo viridi-olivncco; gula alba; gastraeo et subcaudalibus laete flavis; remigibus obscure fuscis, exterius viridi-olivaceo, primariis paullum grisescenti marginatis, intus basin versus et subalaribus albis, flavo tinctis, tectricibus alarum superioribus viridi-olivaceo late marginatis; cauda olivacea, apicen versus nigricanti; rostro nigro; pedibus plumbeis.

Long. tot. $0^{\mathrm{m}}, 160$; al. $0^{\mathrm{m}}, 090$; caud $0^{\mathrm{m}}, 070$; rostri culm. $0^{\mathrm{m}}, 020$; tarsi $0^{\mathrm{m}}, 025$.

Hab. in Papuasia - Ins. Tenimberensibus, Babbar (Riedel).

In ho modificato alquanto la descrizione data dal Mejer di questa specie, giacchè arendo potuto per sua cortesia esaminarne l'esemplare tipico, non ho trovato che le remiganti o le cuopritrici superiori delle ali siano di color fulvo scuro.

Il Meyer dice che questa specie è affine alla $P$. collaris, Ramsay, dalla quale si distingue per diversi caratteri e specialmente per le strie bianche lungo gli steli delle piume nere della fascia pettorale, e per la coda non unicolore, ma olivacea alla base e nereggiante verso l'apice.

\section{(456) Pachycephala innominata, SALVAD.}

Pachycephala innominata, Gad, Cat. B. VIII, p. 197 (1883).

\section{(457) Pachycephala soror, ScLat.}

Pachycephala affinis, Meyer, Sitzb. k. Akad. Wien, LXIX, pp. 69, 392 (1874). - Sclat., Ibis, 1874, p. 417. - Meyer, 1. c. LXX, pp. 70, 128 (nota) (1874). - ? Oust., Bull. Soc. Philom. Paris, 1877, p. 57. - Salvad.. Ann. Mus. Civ. Gen. X, p. 142 (1877), - Id., Orn. Pap. e Mol. II, p. 224 (1881). Pachycephala soror, Gad., Cat. B. VIII, p. 195 (1883), - Guillem.,P. Z. S. 1885, p. 637 (Arfak).Meyer, Zeitschr. f. ges. Orn. III, p. 21 (1886) (Monti Hufeisen).

Pachycephala sp., Meyer, Zeitschr. f. ges. Orn. 1884, p. 200, sp. 14 ( $=$ P. soror, Sclat. juv.).

Il Meyer (Zeitschr.f.ges. Orn. I, p. 200, 1884) sostiene che la sua $P$. affinis (Sitzb. 7. Akad. Wien, LXIX, p. 392, 1874) sia la femamina della P. soror, Sclat. 
e non della $P$. schlegeli, Rosenb., come a me era sembrato facendo il confronto del tipo con le femmine della $P$. schlegeli. Inoltre il Meyer afferma che gli esemplari dei Monti Arfak sembrano più vivamente coloriti di quelli dei Monti Hufeisen, la quale cosa non mi è apparsa esatta, laddove è notevole la differenza nel becco molto piư breve $\left(0^{\mathrm{m}}, 012\right.$, invece di $\left.0^{\mathrm{m}}, 014\right)$ nei due esemplari dei Monti Hufeisen, che ho potuto esaminare per cortesia del Meyer.

\section{Sp. 1098 (457 bis) Pachycephala meyeri, Salvad.}

Pachycephala affinis, Meyer, Zeitschr. f. gez. Orn. I, p. 200, sp. 11 (Arfak) (1884, nec Sitzb.k Akad. Wien, LXIX, p. 392, 1874).

Supra brunnescenti-olivacea, pileo obscure griseo, fronte clariore, lateribus capitis fusco-brunneis; mento, gula et pectore summo sordide albis, torque pectorali obsoleto brunescenti-griseo; gastraeo, abdomine, corporis lateribus et subcaudalibus sulphureis; axillaribus et subalaribus clarioribus; alis et cauda fuscis, remigum et rectricum pogoniis externis olivaceo limbatis; alis subtus fusco-griseis; remigum pogoniis internis albescenti marginatis; rostro nigro; pedibusque in exuvie plumbeis.

Long. tot. circa $0^{\mathrm{m}}, 140(1)$; al. $0^{\mathrm{m}}, 077$; caud. $0^{\mathrm{m}}, 036$; tarsi $0^{\mathrm{m}}, 020$; rostri $0^{\mathrm{m}}, 013$.

Hab. in Papuasia - Nova Guinea, Montibus Arfak (Laglaize, Meyer).

Il tipo di questa specie è una femmina raccolta dal Laglaize, ma il Meyer riferisce alla medesima un'altra femmina della sua collezione, sebbene per talune differenze che essa presenta, egli non possa escludere il dubbio che debba essere riferita ad un'altra specie. Le differenze sarebbero le seguenti : il suo esemplare ha le parti superiori di colore un poco più pallido, il colore bianchiccio della gola non s'arresta nettamente al petto, ore non appare una fascia trasversale, ma passa gradatamente nel color grigio-bruniccio del petto; inoltre i margini interni delle remiganti hanno una leggera tinta giallognola; anche nelle dimensioni havri una piccola differenza: lung. tot. $0^{\mathrm{m}}, 140$; al. $0^{\mathrm{m}}, 077$; coda $0^{\mathrm{m}}, 058$; tarsi $0^{\mathrm{m}}, 0195$; becco $0^{\mathrm{m}}, 0125$.

Ho esaminato ambedue gli esemplari menzionati dal Meyer e credo anche io che appartengano ad una stessa specie, ad onta delle lievi differenze indicate dal Meyer.

La $P$. meyeri, Salvad. (=affinis, fem. Meyer, 1884) differisce dalla $P$. schlegeli fem. pei seguenti rispetti: in questa il bianco sporco della gola non scende tanto in basso, $\mathbf{i}$ margini scuri delle piume presentano un aspetto ondulato, il colore grigio del petto è più cupo e meno bruniccio, si estende meno in basso e passa in un giallo olivaceo-verdognolo, laddove nella $P$. meyeri il giallo è un puro giallo-zolfino; inoltre nella $P$. schlegeli fem. le parti superiori sono più olivacee, la testa è più azzurrognola (plumbea?), le piume auricolari meno diverse dal colore del capo, laddove nella nuova specie le auricolori sono più distintamente brune; finalmente essa differisce per le dimensioni: nella $P$. schelegeli le ali, la coda ed i tarsi sono più lunghi, il becco invece è più corto: al. $0^{\mathrm{m}}, 079-0^{\mathrm{m}}, 082$; coda $0^{\mathrm{m}}, 060-0^{\mathrm{m}}, 062$; tarso $0^{\mathrm{m}}, 022-0^{\mathrm{m}}, 0225$; becco $0^{m}, 011$.

(1) Il Meyer dà mill. 130 pel' la lunghezza totale, ma la pelle è evidentemente accorciata colla preparazione.

[230] 
La $P$. meyeri differisce poi dalla $P$. soror fem. pei seguenti rispetti: questa ha il becco più grande; il colore giallo olivaceo comincia subito sotto il bianco della gola e finalmente le dimensioni sono maggiori : al. $0^{\mathrm{m}}, 087$; cod. $0^{\mathrm{m}}, 0685$; tarso $0^{\mathrm{m}}, 021$; becco $0^{\mathrm{m}}, 014$.

Il Meyer crede probabile che il maschio sconosciuto dalla sua nuova $P$. affinis (=meyeri, Salvad,) abbia la gola bianca, circondata inferiormente da una fascia pettorale nera. Tuttaria non è improbabile che la $P$. meyeri appartenga al sottogenere Hyloterpe, le cui specie non presentano differenze cospicue nei due sessi.

Ton so comprendere come il Meyer abbia proposto di usare per questa sua specie il nome $P$. affinis, già da lui adoperato nel 1874 per la femmina di un'altra specie!

(458) Pachycephala schlegeli, Rosenb.

Pachycephala schlegelii, Salvad., Orn. Pap. e Mol. Il. p. 223 (syn. emend.) (1881). - Gad., Cat. B. VIII, p. 195 (1883. - Meyer, Zeitschr. f. ges. Orn. I, p. 202, sp. 12 e 13 (1884). - Guillem., P. 2. S. 1885, P. 638 (Arfak)

Il Meyer descrive due femmine giovani, che anche io ho esaminato, aventi piu o meno di colore rosso-rugginoso sulla testa, sulla cervice, sul groppone e sulle ali. Inoltre io credo che spetti alla stessa specie un esemplare che egli descrive come giorane di specie ignota; le sue dimensioni corrispondono con quelle della $P$. schlegeli e credo che esso sia in stato di transizione dall'abito giovanile all'abito adulto.

(459) Pachycephala (?) rufinucha, Sclat.

Pachycephala rufinucha, Gad., Cat. B. VIII, p. 211 (1883). - Guillem., P. Z. S. 1885, p. 638 (Arfak).

(460) Pachycephala griseiceps, G. R. GR.

Pachycephala griseiceps, part., Gad., Cat. B. VIII, p. 215 (1883), - Salvad., Ibis, 1884, p. 324. - Nehrk., Journ. f. Orn. 1885, p. 33 (Waigeu). -

Pachycephala jobiensis, Meyer, Zeitschr. f. ges. Orn. 1884, p. 203 (Waigen!) (nec Sitzb. k. Ak. Wiss. Wien, LXIX, p. 394).

L'esemplare di Omka in Waigiou (Laglaize), menzionato dal Meyer col nome di $P$. jobiensis, se pure la località indicata è esatta, mi sembra riferibile alla $P$. griseiceps, specie nota di Waigiou e che somiglia molto alla $P$. jobiensis.

(461) Pachycephala jobiensis, MEYer.

Pachycephala griseiceps, part., Gad. Cat. B. VIII, p. 215 (1883).

Pachycephala jobiensis, Salvad, Ibis, 1884, p. 324.

(462) Pachycephala miosnomensis, SALVAD.

Pachycephala griseiceps, part., Gadow, Cat. B. VIII, p. 215 (1883).

Pachycephala miosnomensis, Salvad, Ibis, 1884, p. 324.

(463) Pachycephala rufipennis, G. R. GR.

Pachycephala rufpennis, Gad, Cat. B. VIII, p. 218 (1883). 


\section{(464) Pachycephala dubia, Ramsay.}

Pachycephala brunnea, Ramsay (nec Wall.). - Sharpe, Gould's B. New Guin. pt. XIV, pI. 11 (1883). - Gad., Cat. B. VIII, p. 219 (1883).

(465) Pachycephala griseonota, G. R. GR.

Pachycephala griseonota, Gad., Gat B. VIII, p. 217 (1883).

(466) Pachycephala cinerascens, Salvad.

Pachycephala cinerascens, Gad., Cat. B. VIII, p. 216 (1883). - Pleske, Bull. Acad. Petersh. XXIX, p. 530 (Ternate) (1884).

\section{(467) Pachycephala phaeonota (S. MüLL.).}

Pachycephala phaeonota, Salvad, Voy. Chall., Birds, p. 65, pl. XVIII, f. 1 (1881). - Gad., Gat. B. VIII, p. 214 (1883). - Pleske, Bull. Acad. Petersb. XXIX, n. 530 (Ternate) (1884).

(468) Pachycephala hyperythra, Salvad.

Pachycephala hyperythra, Sharpe, Gould's B. New Guin. pt. XIV, pl. 12 (1883). - Gad., Cat. B. Vlil, p. 213 (1883).

Lo Sharpe esprime il dubbio che gli esemplari raccolti nei Monti dell'Astrolabio, nella Nuova Guinea meridionale-orientale, possano appartenere ad una specie distinta.

(469) Pachycephala leucogastra, SALv. et D'ALB.

Pachycephala leucogastra, Gad., Cat. B. VIII, p. 222 (1883).

\section{Sp. 1099 (469 bis) Pachycephala arctitorquis, Sclat.}

Pachycephala arctitorquis, Sclat., P. Z. S. 1883, pp. 51, 55, 195, 200, pl. XIII. - Gad., Cat. B. VIII, p. 368 (1883). - Sharpe, Gould's B. Now Guin. pt. XV, pl. 13 (1883). - Meyer, Sitzb。 u. Abh. Gesellsch. Isis, 1884, Abh. I, p. 34. - Forbes, P. Z. S. 1884, pp. 428, 432. - Salvad.. P. Z. S. 1884 , p. 578 .

Pachycephala Riedelii, Meyer, I. c. p. 35 (Timor-laut).

Supra cinerea, interscaputio obsolete fusco striolato; capite nigro cum torque jugulari concolore conjuncto; gula el corpore subtus albis; alis caudaque nigris cincreo limbatis; subalaribus et remigum marginibus interioribus albis; rostro et pedibus nigris.

Foem. Supra fusco-cinerea, sincipite brunescenti; secundariarum pogoniis externis eodem colore, sed vividiore, primariarum fulvescenti marginatis; pectore, abdomine et subcaudalibus fulvo-brunnescenti-albis, gula et pectore nigrescenti striolatis, subalaribus et remigum marginibus interioribus albescentibus; rostro pedibusque nigris.

Juv. Supra mufescens, subtus albida, fusco striolata; rostro pallido.

Long. tot. $0^{\mathrm{m}}, 155-0^{\mathrm{m}}, 140$; al. $0^{\mathrm{m}}, 080-0^{\mathrm{m}}, 078$; caud. $0^{\mathrm{m}}, 069-0^{\mathrm{m}}, 063$; rostri culm. $0^{\mathrm{m}}, 015$; tarsi $0^{\mathrm{m}}, 022-0^{\mathrm{m}}, 021$.

Hab. in Papuasia - Ins. Tenimberensibus, Timor-laut (Forbes, Riedel).

Questa specie somiglia alla $P$. Tencogastra, Salvad. et d'Alb.; il maschio differisce da quello della specie della Nuova Guinea per la fascia giugulare nera più stretta 
e per avere un indistinto collare grigio, che separa il nero della testa dal grigio del dorso.

Il Meyer vorrebbe' che gli esemplari descritti dallo Sclater come femmine di questa specie appartenessero ad una specie distinta ( $P$. riedelii, Meyer), la quale cosa è stata contraddetta dal Forbes ed anche a me non sembra esatta; io credo che la $P$. riedelii, Meyer, sia fondata sopra i giovani della $P$. arctitorquis, la femmina della quale è stata esattamente descritta dal Meyer.

La $P$. arctitorquis, secondo il Forbes, è specie molto comune nelle Isole Tenimber, ove frequenta gli alberi, e talora discende anche sul terreno.

\section{Sp. 1100 (469ter) Pachycephala kebirensis, MEXER (?).}

Pachycophala kebirensis, Meyer, Sitzb. u. Abh. Gesellsch. Isis, 1884, Abh. I, p. 35 (1884) (Babbar) (Tipo esaminato).

Pachycephala arctitorquis, part., Forbes, P. Z. S. 1884, pp. 428, 432.

Foem. Foeminae P. aretitorquis simitis, sed supra olivaceo lavata, subtus albidior, cauda longiore; rostro pallide fusco.

Long. tot. $0^{\mathrm{m}}, 150$; al. $0^{\mathrm{m}}, 080$; caud. $0^{\mathrm{m}}, 071$; rostri $0^{\mathrm{m}}, 015$; tarsi $0^{\mathrm{m}}, 023$.

Hab. in Papuasia - Ins. Tenimberensibus, Babbar seu Kebir (Riedel).

Il Meyer ha descritto una femmina che egli non crede di poter riferire alla $P$. arctitorquis, cui invece la riferisce il Forbes, ma senza avere esaminato il tipo del Meyer. Questi ha avuto la cortesia d'inviarmelo, ma per causa della insufficienza dei materiali di confronto io non sono in grado di decidere la questione.

(470) Pachycephala monacha, G. R. GR.

Pachycephala monacha, Gad., Cat. B, VIII, p. 222 (1883).

(471) Pachycephala leucostigma, Salvad.

Pachycephala leucostigma, Gad., Cat. B. VIII, p. 223 (1883).

(472) Pachycephala spinicauda (J. et P.).

Pachycephala spinicauda, Gad., Cat. B. VIII, p. 182 (nota 1) (1883).

(473) Pachycephalopsis hattamensis (Meyer).

Pachycephala hattamensis, Gad., Cat. B. VIII, pp. 225, 368 (1883).

Pachycephalopsis hattamensis, Guillem, P. Z. S. 1885, p. 638 (Arfal).

\section{Sp. 1101 (473 bis) Pachycephalopsis fortis (Gadow).}

Pachycephala fortis, Gad., Cat. B. Brit. Mus. VIII, p. 369 (1883).

Pachycephalopsis fortis, Sharpe, Gould's B. New Guin, pt. XVI, pl. 5 (1884).

Supra virescenti-olivacea; capite, cervice, lateribus colli, auricularibusque griseoschistaceis; loris griseo-albidis; genis, gula et pectore summo pallide griscis, scapis plumarum colli antici fuscis; abdomine medio albido, lateribus corporis brunnescente-olivaceo tinctis; tibiis griseis, olivaceo tinctis; subcaudalibus flavis; alis fuscis, olivaceo marginatis, remigibus subtus fuscis, intus cinereo marginatis ; cauda fusco - 
olivacea, rectricibus in pogonio externo virescentioribus, obsolete fusco transfasciolatis; rostro fusco; pedibus plumbeis (?).

Long. tot. $0^{\mathrm{m}}, 167$; al. $0^{\mathrm{m}}, 092$; caud. $0^{\mathrm{m}}, 069$; rostri $0^{\mathrm{m}}, 021$; tarsi $0^{\mathrm{m}}, 026$.

Hab. in Papuasia - Nova Guinea meridionali-orientali, in Montibus Astrolabii (Goldie).

Dice lo Sharpe che questa specie è affine alla $P$. hattamensis (Meyer), cui somiglia pel dorso verde-olivaceo e per la testa grigia, ma ne differisce per la gola e per la parte superiore del petto di colore grigio uniforme, pel sottocoda di color giallo-chiaro e per la mancanza di colore rossigno sulle ali.

Devo confessare che, giudicando dalla figura, non mi sembra che questa specie sia una vera Pachycephalopsis, giacchè non scorgo i tarsi lunghetti e la coda piuttosto breve, pei quali caratteri le specie di quel genere differiscono dalle vere $P a$ chycephalae.

(474) Pachycephalopsis albispecularis (SALVAD.).

Pachycephala albispecularis, Gad., lat. B. VIII, p. 212 (1883).

(1002) Pachycephalopsis poliosoma, SHARPE.

Pachycephala poliosoma, Gad., Cat. B. VIII, F. 226, pl. 9 (1883). - Salvad., Ibis, 1884, p. 324. (475) Pachycare flavogrisea (Mexer).

Pachycare flavogrisea, Gad., Cat. B. VIII, p. 227 (1883).-- Guillem., P. Z. S. 1885, p. 638 (Arfak).

(476) Orthonyx novae guineae, Meyer.

Orthonyz novae guineae, Sharpe, Cat. B. VII, p. 672 (1883!

(477) Climacteris placens, SCLAT.

Climacteris placens, Gad., Cat. B. VIII, p. 335 (1889). - Guillem., P. Z. S. 1885, p. 638 (Arfak).

(478) Sittella papuensis (Schleg.).

Sittella papuensis, Gad., Cat. B. VIII, p. 365 (1883). - Guillem., P. Z. S. 1885, p. 638 (Arfak).

Il Guillemard descrive un esemplare senza le macchie (obsolete) bianche sul petto, sull'addome e sulle parti superiori, e senza traccia di bianco sul vessillo interno della $3^{\mathrm{a}}, 4^{\mathrm{a}}$ e $5^{\mathrm{a}}$ remigante primaria.

\section{Sp. 1102 (478 bis) Sittella albifrons, RAMSAY (?).}

Sittella albifrons, Ramsay, Pr. Linn. Soc. N. S. W. VIII, p. 24 (1883). - Salvad., Ibis, 1884, p. 354.

Capite et pectore summo albo-sericeis; supracaudalibus albis, macutis fuscis in medio notatis; cauda fusca; subcaudalibus nigricantibus, maculis apicalibus latis notatis; parte inferiore uropygii alba; rectricibus tribus extimis maculis apicalibus latis notatis, $4^{\mathrm{a}}$ et $5^{\mathrm{a}}$ apice vix maculatis; alis fuscis; remigibus quinque primariis in pogonio interno macula alba parva notatis, unde ala subtus macula alba plus minusve conspicua notata; tectricibus inferioribus remigum primariarum 
albo maculatis; pectore imo ct gastraco reliquo albis, plumis singulis stria lata fusca in medio notatis; dorso et uropygio fuscis, plumis in medio nigro striatis; rostri flavi apice nigro; subalaribus fuscis; pedibus flavis.

Juv. MLagis fuscescens, et colore albo cinereo tincta.

Long. tot. $0^{\mathrm{m}}, 106$; al. $0^{\mathrm{m}}, 086$; caud. $0^{\mathrm{m}}, 042$; rostri $0^{\mathrm{m}}, 012$; tarsi $0^{\mathrm{m}}, 015$.

Hab. in Papuasia - in Nova Guinea meridionali-orientalis in Montibus Astrolabii (Rolles).

Il Kolles ha trovato questa specie sugli altipiani dei Monti dell'Astrolabio a 3000 piedi circa di altezza; essa frequentava in branchetti gli Eucalipti.

Io non riesco ad afferrare le differenze fra questa specie e la S. papuensis (Schleg.), colla quale il Ramsay non la confronta; non è improbabile che sia la stessa specie.

(479) Hermotimia theresia, SALVAD.

Cinnyris theresae, Gad., Cat. B. IX, p. 74 (1884).

(480) Hermotimia maforensis (MEYER).

Cinnyris aspasiae, part., Gad., Cat. B. IX, p. 68 (1884).

Hermotimia maforensis, Salvad., Ibis, 1884, p. 325.

(481) Hermotimia mysorensis (MEYER).

Cinnyris aspasiae, part., Gad., Cat. B. IX, p. 68 (1884).

Hermotimia mysorensis, Salvad., Ibis, 1884, p. 325.

(482) Hermotimia jobiensis (MEYER).

Cinnyris aspasiae, part., Gad., Cat. B. IX, p. 68 (1884:

Hermotimia jobiensis, Salvad., Ibis, 1884, p. 325.

Cinnyris jobiensis, Guiller., P. Z. S. 1885, p. 638 (Jobi).

(483) Hermotimia aspasia (LESS.).

Hermotimia aspasia, Sharpe, Journ. Linn. Soc., Zool. XVI, p. 436 (Morocco, East Cape) (1882).Salvad., Ibis, 1884, pp. 325,326. - Kehrk., Journ. f. Orn. 1885, p. 33 (Waigeu).

? Nectarinia zenobia, Bernst.(nec Less.), in Musschenbr., Dagboek, pp. 148, 151 (Sorong, Seleh) (1883).

Ginnyris aspasiae, part., Gad., Cat. B. IX, p. 68 (1884). - Guillem., P. Z. S. 1885, p. 639 (Waigiou, Mysol).

Fra le localita abitate da questa specie, o razza secondo il Gadow, questi annovera Amboina, dove essa non è stata mai trovata e vive invece la $H$. aspasioides.

(484) Hermotimia cornelia, Salvad.

Cinnyris aspasiae, part., Gad., Cat. B. IX, p. 68 (1884).

Hermotimia cornelia, Salvad, Ibis, 1884, p. 325.

\section{(485) Hermotimia chlorocephala (Salvad.).}

Ginnyris aspasia, part., Gad., Cat. B. IX, p. 68 (1884). - Guillem., P. Z. S. 1886, p. 639 (Q Aru). Hermotimia chlorocephala, Meyer, Zeitschr. f. ges. Orn. I, p. 286 (1884). 
Il Meyer dà le dimensioni di due femmine delle isole Aru, notevoli per la loro piccolezza: al. $0^{\mathrm{m}}, 050-0^{\mathrm{m}}, 052$; coda $0^{\mathrm{m}}, 028$; becco $0^{\mathrm{m}}, 015$. Queste dimensioni sono state da me rerificate in una delle due femmine menzionate.

(486) Hermotimia corinna, SALVAD.

Cinnyris aspasiae, Gad., Cat. B. IX, p. 68 (1884).

Hermotimia corinna, Salvad,, Ibis, 1884, p. 325.

Nectarinia corinna, Finsch, Vög. d. Südsee, p. 10 (1884) (Nova Britannia).

(487) Hermotimia aspasioides (G. R. GR.).

Hermotimia aspasioides, Salvad., Voy. Chall, Birds, p. 63 (1881:- Blas, u. Nehrk, Verh. zo-b. Ges. Wien, 1882, p. 424 (Amboina, nido ed uova). - Salvad., Ibis, 1884, pp. 325, 326.

Cinnyris aspasiae, part., Gad., Cat. R. IX, p. 68 (1884.

Il Gadow ha indicato questa specie, o razza secondo lui, come vivente nella Nuova-Guinea orientale-meridionale e nella Nuova Britannia, invece essa è propria del gruppo di Ceram, ore rappresenta la $H$. aspasia della Nuova-Guinea.

\section{Sp. 1103 (487 bis) Hermotimia cristianae (Tristr.).}

Ginnyris cristianae, Tristr., Ibis, 1889, p. 555.

C. (Hermotimia) H. corinnae similis, sed paullo najor; pileo plus aurato; gutture chalybaeo-caeruleo, minime purpurescente; rostro longissino; rostro pedibusque nigris.

Hab. in ins. St. Aignan dicta (Thomson).

Il Tristram ha ricevuto due maschi di questa specie, e dice che essa somiglia molto alla $H$. aspasioides, dalla quale si può distinguerla immediatamente pel becco molto più lungo che non nelle altre specie e per la colorazione metallica del pileo, che è intermedia fra il verde metallico scuro della $H$. corinna ed il dorato della $H$. auriceps. La gola è di un azzurro metallico splendente, senza alcuna traccia del violetto che si trova in molte altre specie.

(489) Hermotimia proserpina (WALL.).

Cinnyris aspasiae, part., Gad., Cat. B. IX, p. 68 (1884'.

Hermotimia proserpina, Salvad., Ibis, 1884, p. 325.

(489) Hermotimia nigriscapularis, Salvad.

Cinnyris aspasiae, part., Gad., Cat, B. IX, p. 68 11884).

Hermotimia nigriscapularis, Salvad., Ibis, 1884, p. 325.

(490) Hermotimia salvadoril (Shellex).

Cinnyris aspasiae, part., Gad., Cat. B. IX, p. 68 (1884).

Hermotimia salvadorii, Salvad, Ibis, 1884, p. 325.

(491) Hermotimia auriceps (G. R. GR.).

Fermotimia auriceps, Salvad,, Voy. Chall., Birds, p. 61 (1881). — Id., Ibis, 1884, p. 325. - Pleske, Bull. Acad. Petersh. XXIX, p. 530 (Ternate) (1884). - Nehrk., Journ. fo Orn. 1885, p. 33. Guillem., P. Z. S. 1885̄, p. 572 (Ternate, Batchian, Bisa). - Salvad, Ibis, 1886, p. 152. 
Hermotimia aspasiae, part., Gad., Cat. B. IX, p. 68 (1884.

Io ho gia fatto notare che l'esemplare indicato di Waigiou dal Nehrkorn era invece di Galela in Halmahera.

Dice il Guillemard che un esemplare dell'Isola Bisa, nel gruppo di Obi, è noterole pei riflessi bronzati della testa.

\section{(492) Hermotimia morotensis (Shellex).}

Cinnyris aspasiae, part., Gad. Cat. B. IX, p. 68 (1884).

Hermotimia morotensis, Salvad., Ibis, 1884, p. 325.

(493) Cyrtostomus zenobia (LESS.).

Cyrtostomus zenobia, Salvad., Voy. Chall. Birds, p. 62 (1881. - Blas. u. Nehrk., Verb. z,-b. Gesellsch. Wien, 1882, p. 425 (Amhoina) - Blas., P. Z. S. 1882, p. 708 (Ceram!

Ginnyris zenobia, Gad., Cat. B. IX, p. 90 (1884).

(494) Cyrtostomus frenatus (S. MüLL.).

Nectarinia frenata, Sclat., Voy. Chall., Birds, p. 28 (1881). - Finsch, Vög. d. Südsee, p. 9 (Nova Britannia), pp. 25, 32 (1884).

Cyrtostomus frenatus, Salvad., Voy. Chall., Birds, pp. 61,80 (1881). - Meyer, Sitzb. u. Abh. Gesellsch. Isis, 1884, Abh. I, p. 37 (nido ed uova). - Id., Zeitschr. f. ges. Orn. I, p. 287 (1884).

- Pleske, Bull. Acad. Petersh. XXIX, p. 531 (uovo) (Ternate) (1884). - Nebrk., Journ. f. Orn. 1885, p. 33 (Waigeu). - Meyer, Zeitschr. f. ges, 0rı。1886, p. 35 (Tarawai).

Cinnyris frenata, Ramsay, Pr. Linn. S. N. W. VII, p. 28 (1882) (Isole Salomone). - Tristr,, lbis, 1882, pp. 135, 142. - Ramsay, Tab. List. p. 14 (1888).

Cinnyris jugularis, part., Gad., Cat. B. IX, p. 84 (1884). - Salvad., lbis, 1884, p. 325.

Cinnyris frenatus, Guillem., P. Z. S. 1885, pp. 572 (Ternate, Batchian), 639. - Grant, P. Z. S. 1888, p. 197 (Aola).

(496) Dicaeum vulneratum, WALL.

Dicaeum vulneratum, Salvad., Voy. Chall., Birds, p. 63 (1881). - Blas. u. Nerk., Verh. zo-b. Gesellsch. Wien, 1882, p. 425. - Sharpe, Cat. B. X, p. 29 (1885).

(497) Dicaeum layardorum, SALVAD.

Dicaeum layardorum, Sharpe, Cat., B. X, p. 32 (1885).

(498) Dicaeum schistaceiceps, G. R. Gr.

Dicaeum schistaceiceps, Sharpe, Cat. B. X, p. 28 (1885).

(499) Dicaeum erythrothorax, LEss.

Dicaeum erythrothorax, Sharpe, Cat. B. X, p. 31 (1885).

(500) Dicaeum pectorale, Müll. et Schleg.

? Dicaeum personatum, Bernst, in Musschenbr., Dagboek, p. 149 (Sorong) 1883).

? Dicaeum papuense, Bernst. (nec Gm.), op. cit. p. 156 (Kalwal) (1883).

Dicaeum pectorale, Nehrk, Journ. f. Orn. 1885, p. 33 (Waigeu). - Sharpe, Cat. B. Brit. Mus: X, p. 29 (1885). - Guillem., P. Z. S. 1885, p.639. - Sharpe, Gould's B. New. Guin. pt. XXI, pl. 12 (1886).

(501) Dicaeum maforense, SALVAD.

Dioaeum matorense, Sharpe, Cat. B. X, p. 33 (1885). 
(502) Dicaeum mysoriense, SALVAD.

Dicaeum mysoriense; Sharpe, Cat. B. X. P. 34 (1885).

(503) Dicaeum joblense, Salyad.

Dicaeum geelvinkianum, Sharpe, Cat. B. X, p. 34 (1885!

Sp. 1104 (503 bis) Dicaeum nitidum, Trisrr.

Dicaeum nitidum, Tristr., Ihis, 1889, p. 555.

Dicaeum D. rubro-coronato statura et coloribus simillimum, sed dorso, scapularibus et remigibus viridi-olivaceis, nec purpureo-caeruleis, diversum.

$H a b$. in insula Sudest dicta (Thomson).

Il Tristram ha ricevuto un esemplare di questa specie.

(504) Dicaeum rubro-coronatum, SHARpe.

Dicaeum rubro-coronatum, Sharpe, Journ. Linn. Soc., Zool. XVI, p. 436 (Morocco) (1882). - Id., Gat. B. X, p. 26, pl. I, f. 1 (1886,

Sp. 1105 (504 bis) Dicaeum pulchrius, SHARPE.

Dicaeum rubro-coronatum, Sharpe, Journ. Linn. Soc.. Zool. XVI, p. 436 (1882) (nec Sharpe 1876) Dicaeum pulchrius, Sharpe, P. Z. S. 1883, p. 579. - Id., Cat. B. X, p. 27, pl. I, f. 2 (1885).

Dicaeum simile D. rubro-coronato, sed pilei colore scarlatino magis extenso et usque ad mucham producto et praecipue corporis lateribus flavicanti-olivaceis, nec cinereis, distinguendum (Sharpe).

Long. tot. $0^{\mathrm{m}}, 081$; al. $0^{\mathrm{m}}, 064$; caud. $0^{\mathrm{m}}, 025$; rostri $0^{\mathrm{m}}, 010$; tarsi $0^{\mathrm{m}}, 010$.

$H a b$. in Papuasia - Nova Guinea meridionali-orientali, in montibus Astrolabii (Goldie).

(505) Dicaeum rubrigulare, D'Alb. et SALvad.

Dicaeum rubrigulare, Sharpe, Cat. B. X, p. 28 (1885).

(506) Dicaeum albo-punctatum, D'ALB. et SALVAD.

Dicaeum albo-punctatum, Sharpe, Cat. B. X, p. 28 (188i).

Sp. 1106 (506 bis) Dicaeum salvadoril, MEYER.

Dicaeum salvadorii, Meyer, Silzb. u. Abh. Gesellsch. Isis, 1884, Abh. I, p. 38 (1884) (Ins. Babbar) (Tipo esaminato). - Sharpe, Cat. B. X, p. 26 (1885.

Supra nigro-caeruleum, nitens; supracaudalibus, gula pectoreque pulchre rubris; subtus albidum, stria media gastraei lata nigra; subalaribus albis; rostro pedibusque vigris.

Long. tot. $0^{\mathrm{m}}, 085$; al. $0^{\mathrm{m}}, 058$; caud. $0^{\mathrm{m}}, 033$; rostri $0^{\mathrm{m}}, 010$; tarsi $0^{\mathrm{m}}, 015$. Hab. in Papuasia - Ins. Babbar (Riedel).

Dice il Meyer che questa specie somiglia al D. mackloti, Müll. di Timor, ma [238] 
che ne differisce per le ali più lunghe, pel colore rosso della gola discendente più in basso sul petto e più ocraceo, essendo le piume bianche alla base, poi gialle ed all'estremità rosse; nelle piume del $D$. mackloti, manca il giallo.

Ho esaminato il tipo di queste specie, ma non ho potuto confrontarlo con esemplari della specie di Timor.

(507) Dicaeum ignicolle, G. R. GR.

Dicaeum ignicolle, Meyer, Zeitschr. f. ges. Urn. I, p. 282 (1884). - Sharpe, Cat. B. X, p. 21 (1885).

(508) Dicaeum kelense, Saltad.

Dicaeum keiense, Sharpe, Cat. B. X, p. 22 (1885).

\section{Sp. 1107 (508 bis) Dicaeum fulgidum, Sclat.}

Dicaeum fulgidum, Sclat., P. Z. S. 1883, pp. 51, 56, 200. - Sharpe, Gould's B. New. Guin. pt. XV, pl. 6 (1883). - Forbes, P. Z. S. 1884, p. 432. -- Meyer, Zeitschr. f. ges. Orn. I, p. 194 (1884) Sharpe, Cat. B. X, p. 22 (1885). - Büttik., Not. Leyd, Mus. VIII, p. 64 (1886).

Supra nitide purpurescenti-nigrum; subtus album, coccineo perfusum; hypochondriis olivaceo mixtis; subalaribus et remigum pogoniis internis albis, rostro et pedibus nigris.

Foem. Supra fusco-olivacea, capite grisescente; alis nigris, fusco olivaceo marginatis; rectricibus nigris, olivaceo marginatis; loris obsolete albis; auricularibus olivaceo-cinereis; genis et gula albidis, pectore et abdomine ochraceo tinctis; subcaudalibus pallide rubro tinctis; subalaribus albis, axillaribus ochraceo tinctis; remigibus subtus fuscis, margine pogonii interni albo; rostro, pedibus et iride nigris.

Long. tot. $0^{\mathrm{m}}, 089$; al. $0^{\mathrm{m}}, 054$; caud. $0^{\mathrm{m}}, 029$; rostri $0^{\mathrm{m}}, 010$; tarsi $0^{\mathrm{m}}, 013$.

Hab. in Papuasia - Ins. Tenimberensibus, Larat, Loetoe (Forbes, Riedel).

Lo Sclater ha descritto il maschio e lo Sharpe anche la femmina; il primo dice che questa specio somiglia al $D$. ignicolle ed al $D$. keiense, ma che ne differisce per avere tutto il ventre tinto di rosso.

Secondo lo Sharpe il carattere distintivo del $D$. fulgidum dal $D$. keiense sarebbe nel colore dei fianchi, olivaceo in questo ed ocraceo in quello.

Il Büttikofer, il quale sembra ignorare la pubblicazione del vol. X del Catalogue of Birds, pretende che una femmina di questa specie non sia punto differente dal maschio del $D$. heiense. È a desiderarsi che ulteriori confronti facciano conoscere meglio le differenze fra le due specie.

(509) Dicaeum eximium, Sclat.

Dicaeum eximium, Sharpe, Cat. B. X, p. 33 (1885)

(510) Dicaeum aeneum, JACQ. et PuCHER.

Dicaeum aeneum, Ramsay, Pr. Linn. Soc. N. S. W. VII, p. 28 (1882) (Isole Salomone). - Tristr., Ibis, 1882, p. 142. - Sharpe, P. Z. S. 1883, p. 579. - Id., Gould's B. New Guin. 'nt. XXII, pl. 11 (1884). - Id., Cat. B. X, p. 30 (1885) - Grant, P. Z. S. 1888, p. 197. 
Sp. 1108 (510 bis) Dicaeum tristrami, Sharpe.

Dicaeum tristrami, Sharpe, P. Z. S. 1883, p. 579. - Id., Gould's B. New Guin. pt. XVIII, pl. 5 (1884). - Id., Cat. B. X, p. 34 (1885).

Supra fusco-brunneum; plumarum dorsi marginibus albidis: alis fusco-nigris, remigibus secundariis ultimis dorso concoloribus; supracaudatibus et cauda nigricantibus; plumis frontis, supercilionm et auricularibus conspicue albido marginatis; loris, genis anticis et mento nigricantibus; genis posticis, gula et collo antico albidis, plumarum basi fusca; lateribus pectoris summi brunneis, plumarum marginibus albidis; pectore medio, abdomine et subcaudalibus albis; lateribus cinereis; axillaribus et subalaribus albis; remigibus subtus fuscis, intus albidis; rostro et pedibus nigris; iride grisea.

Long. tot. $0^{\mathrm{m}}, 090$; al. $0^{\mathrm{m}}, 058$; caud. $0^{\mathrm{m}}, 039$; rostri $0^{\mathrm{m}}, 011$; tarsi $0^{\mathrm{m}}, 014$.

Hab. in Papuasia - Ins. Salomonis, San Cristoval (Richards).

Come fa notare lo Sharpe, questa specie si allontana da tutte le altre del genere Dicaeum pel suo modo di colorazione; essa ̀̀ notevole pel colorito bruno dello parti superiori, e per la faccia bianchiccia.

(511) Melanocharis unicolor, SALtAD.

Melanocharis unicolor, Sharpe, Cat. B. X, p. 82 (1885).

(994) Melanocharis bicolor, Ramsay.

Melanocharis bicolor, Ramsay, Pr. Linn. Soc. N. S. W. VIII, p. 24 (1883). - Sharpe, Gat, B. X, p. 81 (1885).

(513) Melanocharis nigra (Less.).

Melanocharis nigra, Nehrk., Journ. f. Orn. 1885, p. 33 (Waigeu). - Sharpe, Cat. B. X. p. 80 (1885).

(514) Melanocharis chloroptera, SALVAD.

Melanocharis chloroptera, Mejer, Zeitschr. f. ges. Urn. I, p. 287 (1884) - Sharpe, Cat. B. X, p. 80 (1885).

(515) Urocharis longicauda, SALVAD.

Urocharis longicauda, Sharpe, lat. B. Brit. Mus. X, p. 79 (1885). - Id., Gould's B. New Guin. pt. XIX, pl. 5 (1885).

(516) Pristorhamphus wersteri, Finsch.

Pristorhamphus wersteri, Sharpe, Cat. B. Brit. Mus. X, p. 82 (1885). - Id., Gould's B. New Guin. pt. XIX, pl. 4 (1885). - Guillem., P. Z. S. 1885, p. 639 (Arfak).

Il Guillemard afferma che una femmina della sua collezione non ha traccia dello macchie bianche sulla coda; essa merita di essere ulteriormente studiata.

\section{(517) Rhamphocharis crassirostris, SALVAD.}

Rhamphocaris crassirostris, Sharpe, Cat, B. Brit. Nus. X, p. 84 (1885) - Id., Gould's B. New Guin. pt. XX, pl. 6 (1885).

[240] 
(518) Oreocharis arfaki (MEYER).

Oreocharis arfaki, Sharpe, Cat. B. X, p. 53 (1885). - Guillem., P. Z. S. 1885, p. 639 (Arfak).

(519) Myzomela nigrita, Gray.

Myzomela meyeri, Salvad, Ann. Mus, Civ. Gen. VII, p. 947 (1875)

Myzomela nigrita, Salvad., Voy. Chall., Birds, p. 80 (1881). — Sharpe, Journ. Linn. Soc., Zool.XVI, p. 437 (Choqueri) (1883)。 - Gad., Cat. B. Brit. Mus. IX, p. 139 (1884). - Meyer, Zeitschr. f. ges. Orn. I, p. 287 (1884). - Guillem., P. Z. S. 1885, p. 640. - Sharpe, Gould's B. New Guin. pt. XXIII, pl. 7 (1887).

\section{SP. 1109 (519 bis) Myzomela ramsayi, Finsch.}

Myzomela Ramsayi, Finsch, Zeitschr. f. ges. Orn. III, p. 21 (1886.. - Sharpe, Gould's B. New Guin. pt. XXIII (nel testo della $M$, nigrila) (1887).

Myzomela M. nigritae, Gr. simillima, sed obscure fumigata.

Long. tot. $0^{\mathrm{m}}, 110$; al. $0^{\mathrm{m}}, 064$; caud. $0^{\mathrm{m}}, 044-0^{\mathrm{m}}, 048$; rostri $0^{\mathrm{m}}, 014$; tarsi $0^{\mathrm{m}}, 015-0^{\mathrm{m}}, 016$.

Hab. in Papuasia - Nova Hibernia, ins. Kapaterong et Nusa (Finsch).

Questa specie differisce dalla $M$. nigrita della Nuova Guinea pel colore nerobruniccio, o fuligginoso, cospicuo specialmente sul dorso e sulle ali. Inoltre, secondo il Finsch, la femmina è simile al maschio e manca del rosso che si trova sulla fronte e sulla gola della femmina della $M$. nigrita.

(520) Myzomela pammelaena, Sclat.

Myzomela pammelaena, Sclat., Voy. Chall., Birds, p. 28, pl. VII. f. 3 (1881). - ? Ramsay, Pr. Linn. Soc. N. S. W. VII, pp. 27, 268 (1882) (Isole Salomone). - Gad., Gat. B. IX, p. 140 (partim) (1884).

Gli esemplari di Ugi e di St. Cristoval nelle Isole Salomone attribuiti a questa specie, giudicando dalla descrizione del Ramsay, sono più piccoli di quelli delle isole dell'Ammiragliato:

Lungh. tot. $0^{\mathrm{m}}, 106$; al. $0^{\mathrm{m}}, 067$; coda $0^{\mathrm{m}}, 048$; becco $0^{\mathrm{m}}, 020$; tarso $0^{\mathrm{m}}, 019$.

\section{(1003) Myzomela tristrami, RAMSAY.}

Myzomela tristrami, Ramsay, Pr. Linn. Soc. N. S. W. VII, p. 26 (1882) (Isole Salomone).

Myzomela pammelaena, Ramsay (nec Sclat.), 1. c. p. 27 (Ugi and St. Cristoval) (1882). - Gad., Cat. B. IX, p. 140 (partim) (1884).

Alla $M$. tristrami vengono attribuiti gli esemplari delle Isole Salomone colla base del becco gialla, ed alla M. pammelaena, Sclat. quelli, pure delle Isole Salomone, col becco tutto nero; credo che sarebbe utile di confrontare gli ultimi coi tipi della $M$. pammelaena, Sclat. delle Isole dell'Ammiragliato.

(521) Myzomela forbesi, RAMSAy.

Myzomela forbesi, Gadow, Cat. B. IX, p. 135 (1884). - Tristr., Ibis, 1885, p. 556 (Fergusson Island). Hab. in ins. Fergusson (Thomson). 
(522) Myzomela rosenbergi, Schleg.

Myzomela rosenbergi, Gad., Cat. B. IX, p. 137 (1884). - Hamsay, Pr. Linn. Soc. N. S. W. X, p. 244 (1885). - Guillem., P. Z. S. 1885, p. 640 (Arfak).

(1004) Myzomela erythromelas, SaLvad.

Myzomela guentheri, Gad., Cat. B. Brit. Mus. IX, p. 129, pl.3 (1884). - Salvad., Ibis, 1884, p. 326. Io ho mostrato che la $\boldsymbol{M}$. guentheri, Gadow, è identica colla mia specie.

Sp. 1110 (1004 bis) Myzomela rubro-cucullata, TRISTR.

Myromela rubro-cucullata, Tristr., Ibis, 1889, p. 228.

Mas capite, dorso et uropygio coccineis; atis, cauda et corpore subtus toto cum macula anteoculari intense, nec fuliginose, nigris; remigum margine interna arcte albida; rostro pedibusque nigris.

Long. tot. $0^{\mathrm{m}}, 130$; al. $0^{\mathrm{m}}, 072$; caud. $0^{\mathrm{m}}, 057$; rostri $0^{\mathrm{m}}, 019$; tarsi $0^{\mathrm{m}}, 017$.

Hab. in ins. Ludoricianis, St.-Aignans (Richards).

Dice il Tristram che questa specie somiglia alla $M$. lifuensis, Layard, ma che ne differisce per essere un terzo più grande, raggiungendo le dimensioni delle specie maggiori; inoltre il colore rosso della testa non è separato, come nella $M$. lifuensis, da un collare nero, ma si continua senza interruzione col rosso del mezzo del dorso, sulla gola non discende tanto in basso ed è molto nettamente definito.

L'esemplare tipo di questa specie fu raccolto nelle Isole Luisiadi (St.-Aignans) dal Richards ed inviato al Tristram.

\section{(1005) Myzomela pulcherrima, Ramsay.}

Myzomela pulcherrima, Ramsay, Pr. Linn. Soc. N. S. W. VII, pp. 27, 673 (1882). - Sclato, P. Z. S. 1883, p. 347 (Ugi). - Gad., Gat. B. IX, p. 131 (1884).

Il Ramsay fa notare che in alcuni esemplari il colore rosso dell'addome e dei fianchi si estende anche sul sottocoda. Inoltre egli descrive un giovane, che suppone di questa specie: Tutte le piume del corpo e della testa di color scuro tinte di rossobruniccio; sulla fronte, sui lati della testa, sulla gola, sul petto, sul dorso e sul sopraccoda le piume hanno gli apici di color rosso più vivo; coda ed ali bruno-nericcie, marginate esteriormente, come anche le scapolari, di olivaceo; cuopritrici superiori delle ali marginate di rosso-bruniccio; sottocoda bruno-rossigno: cuopritrici inferiori dello ali e margine interno delle remiganti verso la base bianchi; becco bruno-nericcio; angolo della bocca giallo; piedi plumbei.

Lungh. tot. $0^{\mathrm{m}} ; 116$; al. $0^{\mathrm{m}}, 061$; coda $0^{\mathrm{m}}, 038$; becco $0^{\mathrm{m}}, 017$; tarso $0^{\mathrm{m}}, 017$.

\section{(523) Myzomela cruentata, Meren.}

Myzomela cruentata, part., Gad., Cat. B. IX. p. 140 (1884) - Salvad., Ibis, 1884, p. 32 . Meyer, Zeitschr. f. ges. Orn. III, p. 22 (Mønti Hufeisen) (1886).

\section{Sp. 1111 (523 bis) Myzomela wakoloensis, Forbes.}

Myzomela wakoloensis, Forbes, P. Z. S. 1883, pp. 115, 583. - Sharpe, Gould's B. New Guin. pt. XVIII, pl. 4 (1884). - Gad., Cat. B. IX, p. 141 (1884) - Forbes, P. Z. S. 1884, p. 429. 
Coccinea; dorsi plumarum, scapularibusque basi nigra, apice late coccineo; loris nigris; alis et cauda nigris, tectricibus minoribus et mediis alarum nigris, mediis interioribus apice rubris, majoribus. et remigibus nigris, olivaceo exterius limb̆atis; regione anali et subcaudalibus pallide olivaceo-flavidis, in medio fuscis, interdum paullum rubro tinctis; tectricibus alarum inferioribus et axillaribus albis; margine interno remigum albidis; rostro nigro, mandibulae basi flavida; pedibus fusco-griseis; iride brunnea.

Foem. Griseo-fusca, fronte, genis, gula et uropygio rubro tinctis; subtus rufoflavescens.

Long. tot. $0^{\mathrm{m}}, 091$; al. $0^{\mathrm{m}} ; 052$; caud. $0^{\mathrm{m}}, 033$; rostri $0^{\mathrm{m}}, 012$; tarsi $0^{\mathrm{m}}, 015$.

Hab. in Moluccis - Buru (Forbes).

Secondo lo Sharpe, questa specie somiglia alla $\boldsymbol{M}$. cruentata, Meyer, più che non ad altra specie, ma ne differisce pel color rosso di tinta diversa, per le ali nere colle remiganti marginate di olivaceo e per la coda nera, senza tinta rossa.

(524) Myzomela coccinea, Ramsay.

Myzomela cruentata, part., Gad., Cat. B. IX. p. 140 (1884). - Salvad., Ibis, 1884, p. 327. Myzomela erythrina, part., Sharpe, Gould's B. New Guin..pt. XVII, pl. 9, fig. maior (1884).

\section{(525) Myzomela erythrina, RAMSAY.}

Myżomela cruentata, part., Gad., Cat., B. IX, p. 140 (1884)。 - Salvad., Ibis, 1884, p. 826. Myzomela erythrina, part., Sharpe, Gould's B. New Guin. pt. XVII, pl. 9, fig. minor (1884)。

Io inclino a considerare la $\boldsymbol{M}$. coccinea delle Isole del Duca di York e la $\boldsymbol{M}$. erythrina della Nuova Irlanda come specificamente distinte.

\section{Sp. 1112 (525 bis) Myzomela kleinschmidti, SHARPE.}

Myzomela cruentata, part., Gad., Cat. B. IX, p. 140 (1884).

Myzomela kleinschmidti, Sharpe, Gould's B. New Guin. pt. XVII (nel testo della M.erylhrina)(1884).

IIyzomela simitis M. erythrinae (an potius M. coccineae, Ramsay?), sed capite et gula obscurioribus et axillaribus subalaribusque infuscatis et rubro tinctis distinguenda.

Hab. in Papuasia - Nova Britannia (Kleinschmidt).

Lo Sharpe fa notare che questa specie è affine alla M. erythrina, part., Sharpe, (=coccinea, Ramsay), dalla quale differisce pei caratteri sopra indicati; nella specio del Ramsay le cuopritrici inferiori delle ali e le ascellari sono di color grigio-torreo, appena tinte di rosso.

Gli esemplari della Nuova Britannia, menzionati dal Gadow, come spettanti alla $\boldsymbol{M}$. cruentata, Meyer, molto probabilmente appartengono alla $\boldsymbol{M}$. kleinschmidti.

(526) Myzomela sclateri, Forbes.

Myzomela sclateri, Gad。, Cat. B. IX, p. 141 (1884).

(527) Myzomela lafargei, P. et J.

Myzomela lafargei, Tristr., lbis, 1882, p. 142. - Gad., Cat. B. IX, p. 135 (nota) (1884).

La patria di questa specie è sempre incerta. 
(528) Myzomela boíei, S. MüLL.

Myzomela boiei, Salvad., Voy. Chall., Birds, p. 66 (1881). - Gad, Cat. B. IX, p. 133 (1884).

(529) Myzomela adolphinae, SAlvad.

Myzomela adolphinae, Gad., Cat. B. IX, p. 134 (1884). - Salvad, Ibis, 1882, p. 326. - Guillenı., P. Z. S. 1885, p. 640 .

Questa specie non è, come vorrebbe il Gadow, una rappresentante della M. erythrocephala sui Monti Arfak, appartenendo ad un gruppo differente, che comprende la M. boiei, la $M$. chloroptera e la $M$. annabellae.

\section{Sp. 1113 (529 bis Myzomela annabellae, ScLAT.}

Myzomela annabellae, Sclat., P. Z. S. 1883, pp. 51, 56, 200. - Sharpe, Gould's B. New Guin pt. XV. pl. 7 (1883). - Gad., Cat. B. IX, p. 13' (1884). - Forbes, P. Z. S. 1884, p. 432. - Meyer, Sitzb. u. Abh. Gesellsch. 1sis, 1882, Abh. I, p. 40 (Babbar). - Id., Zeitschr. f. ges, Orn. I, p. 194 (1884).

Capite, collo, uropygio et supracaudalibus coccineis; loris nigris; dorso, alis et cauda nigris, remigibus primariis exterius olivaceo limbatis; remigibus intus albido. limbatis; pectore summo nigro, gastraeo reliquo pallide olivaceo flavido, lateribus cinereo mixtis; subcaudalibus albo flavidis, in medio obsolete fuscis; subalaribus et axillaribus albis; rostro nigro; pedibus fusco-virescentibus; iride brunnea.

Long. tot. $0^{\mathrm{m}}, 091$; al. $0^{\mathrm{m}}, 050$; caud. $0^{\mathrm{m}}, 033$; rostri $0^{\mathrm{m}}, 014$; tarsi $0^{\mathrm{m}}, 015$.

Hab. in Papuasia - in Ins. Tenimberensibus, Loetoe (Forbes), Babbar (Riedel).

Questa specie somiglia alla $M$. adolphinae, Salvad. della Nuova Guinea, ma ne differisce per avere il dorso ed il petto nero e non grigio scuri un po' olivacei; inoltre le sue dimensioni sembrano alquanto minori.

\section{(530) Myzomela erythrocephala, GodLd.}

Myzomela erythrocephala, part., Gad., Cat. B. IX, p. 133 (1884). - Kamsay, Tab. List, p. 14.

(531) Myzomela infuscata, Salvad.

Myzomela erythrocephala, part, Gad., Cat. B. IX, p. 133 (1884). - Guillem. (nec Gould), P. Z. S. 1885, p. 640 (Aru).

Myzomela infuscata, Salvad., Ibis, 1884, p. 326.

Questa specie è stata ommessa dal Gadow.

\section{(532) Myzomela eques (Less.).}

Myzomela eques var., Ramsiy, Pr. Linn. Soc. N. S. W. VIII, p. 19 (1883). - Salvad., Ibis, 1884, p. 324. Myzomela eques, Gad., Cat. B. IX, p. 141 (1884). - Guillem., P. Z. S. 1885. p. 640 (Mysol).

Il Ramsay fa notare che la femmina degli esemplari della Nuova Guinea orientale-meridionale differisce dal maschio per non avere il colore rosso sulla gola; ma io dubito che la supposta femmina appartenga alla $M$. obscura.

(533) Myzomela obscura, Gould.

My rela obscura, Sharpe, Journ. Linn. Soc., Zool, XVI, p. 437 (East lape) (1882). - Gad., Cat. B. IX, p. 143 (1884). - Finsch, Vög. d. Südsee, p. 33 (1884)。 - Meyer, Zeitschr. f. Æes, Orn. I. p. 287 (1884. - Guillem., P. Z. S. 1885, p. 640 (Aru). - Rimsay, Tab. List, p. 14 (1888). 


\section{(534) Myzomela cineracea, ScLat.}

Myzomela cineracea, Gad., Cat. B. IX, p. 143 (1884). - Finsch, Vög. d. Südsee, p. 10 (Nova Britannia, Ins. Ducis York) (1884).

(535) Myzomela simplex, G. R. GR.

Myzomela simplex, Gad, Cat. B. IX, p, 143 (1884). - Pleske, Bull. Acad. Petersb. XXIX, p. 531 (Ternate) (1884).

(536) Myzomela rubrotincta, Salvad.

Myzomela rubrotincta, Gad., Cut. B. IX, p. 142 (1884). - Salvad, Ibis, 1884, p. 327.

Tanto questa, quanto la specie seguente vengono considerate dal Gadow come sottospecie della $\boldsymbol{M}$. simplex, dalla quale ambedue sono perfettamente distinte.

(537) Myzomela rubrobrunnea, Meyer.

Myyzomela rubrobrunnea, Gad; Cat. B. IX, p. 142 (1884). - Salvad., lbis, 1884, p. 327.

(495) Myzomela melanocephala (Ramsax).

Ginnyris melanocephalus, Ramsay, Pr. Linn. Soc. N. S. W. VII, p. 28 (1882) (Isole Salomune). Cyrtostomus melanocephala, Salvad., Ibis, 1884, p. 326.

Myromela melanocephala, Sharpe, Gould's B. New Guin. pt. XVII, pl. 10 (1884).

Mi unisco ora allo Sharpe nel riferire questa specie al genere Myzomela.

\section{Sp. 1114 (495 big) Myzomela sharpei, Grant (?).}

Myzomela sharpii, Grant, P. Z. S. 1888, p. 197, pl. X, f. 3. - Ibis, 1889, p. 582.

Fronte, genis et gula fusco-nigris; occipite, cervice et dorso olivacco-flavis, uropygio et supracaudalibus olivaceo-rufis; collo et pectore summo olivaceo-flavis, dorso concoloribus, gastraeo reliquo flavicanti-rufo; tectricibus alarum, remigibus rectricibusque nigricantibus, olivaceo-flavo marginatis, primariis intus basin versus albo marginatis; rostro nigro; pedibus olivaceis; iride nigra.

Long. tot. $0^{\mathrm{m}}, 129$; al. $0^{\mathrm{m}}, 064$; caud. $0^{\mathrm{m}}, 045$; rostri $0^{\mathrm{m}}, 021$; tarsi $0^{\mathrm{m}}, 022$.

Hab. in Papuasia - Ins. Salomonis, Guadalcanar (Woodford).

Il tipo di questa specie è stato raccolto presso Aola, nell'isola Guadalcanar.

Mi sorprende che l'Ogilvie-Grant dica essere questa specie affatto diversa da tutte le altre conosciute, laddove essa ha la piu grande somiglianza colla $\boldsymbol{M}$. melanocephala (Ramsay) figurata dallo Sharpe (l. c.); anzi mi viene il dubbio che non ne sia specificamente diversa; confrontando la figura data dall'Ogilvie-Grant con quella della $M$. melanocephala data dallo Sharpe, quella appare diversa per la minore estensione del nero sulla testa, arrestandosi essa alla metà del vertice, laddove nella seconda il nero ricopre tutta la testa; inoltre il colore olivaceo nella $M$. sharpei ha una tinta bruna, che manca nell'altra, ma potrebbe essere che quelle differenze dipendano dall'età; discorrendo della specie precedente, io feci notare di avere esaminato un esemplare che areva le piume del groppone e del sopraccoda tinte di rossigno. 
(538) Glycyphila modesta, G. R. GR.

Glycyphila modesta, Gad., Cat. B. IX, p. 215 (1884). - Meyer, Zeitschr, f. ges. Orn. I, p. 288, Taf. XVIII, f. 7-8 (uova) (1884). - Guillein., P. Z. S. 1885, p. 641 (Aru). - Ramsay, Tab. List, p. 12 (1888).

Il Meyer descrive anche il nido.

\section{(539) Glycyphila nisoria (MüLL.).}

Glycyphila nisoria, Gad., Cat. B. IX, p. 218 (1884).

(540) Conopophila albigularis (GouLd).

Entomophila albigularis. Gad., Cat. B. IX, p. 219 (1884),

Entomophila (Conopophila) albigularis, Ramsay, Tab. List, p. 13 (1888).

(541) Glycychaera fallax, SALvad.

Glycychaera fallax, Meyer, Zeitschr. f. ges. Orn. I, p. 288 (1884' - Sharpe, Gould's B. New Guin. pt. XIX, pl. 6 (1885).

Glycyphila fallax, Gad., Cat. B. Brit. Mus. IX, p. 213 (1884).

(542) Glycychaera poliocephala, SALVAD.

Glycyphila poliocephala, Gad., Cat. B. IX, p. 213 (1884).

(543) Oedistoma pygmaeum, Saltad.

Oedistoma pygmaeum, Gad., Cat. B. Brit. Mus. IX, p. 293 (1884). - Salvad., Ihis, 1884, p. 328. - Sharpe, Gould's B. New Guin. pt. XX, pl. 4 (1885).

(544) Melilestes megarhynchus (G. R. GR.).

Melilestes megarhynchus, Sharpe, Journ. Linn. Soc., Zool. XVI, p. 437 (1882) (Morocco). Meyer, Zeitschr. f. ges. Orn. I, p. 288 (1884). - Nehrk., Journ. f. Orn. 1885, p. 33 (Waigeu! Guillem., P. Z. S. 1885, p. 641 (Waigiou).

Ptilotis megalorhynchus, Gad., Cat. B. IX, p. 218, pl. V (1884).

$H a b$. in Nova Guinea meridionali-orientali (Goldie).

(545) Melilestes novae guineae (Less.).

Arachnothera novae guineae, Gad., Cat. R. IX, p. 110 (1884).

Melilestes novae guineae, Salvad., Ibis, 1884, p. 328. - Meyer, Zeitschr. f. ges. Orn. I, p. 289

(1884) (nido ed uova). - Nehrk., Journ. f. Orn. 1885, p. 33 (Waigeu). - Guillem., P. Z. S. 1885, p. 641 (Waigiou).

(546) Melilestes iliolophus, SALVAD.

Arachnothera iliolophus, part., Gad., Cat. B. Brit. Mus. IX, p. 111, pl. I, f. 2 (1884).

Melilestes iliolophus, Salvad., lbis, 1884, p. 328. - Sharpe, Gould's B. New Guin. pt. XXIII, pl. 8 (1887)

\section{(547) Melilestes affinis, Salvad.}

Arachnothera iliolophus, part., Gad., Cat. B. IX, p. 111 (1884).

Melilestes affinis, Salvad., Ibis, 1882, p. 328.

\section{(1006) Melilestes poliopterus, SHarpE.}

Melilestes poliopterus, Sharpe, Gould's B. New Guin. pt. XIV, pl. 8 (1883). - Salvad., Ibis, $1884, \mathrm{p} .328$.

Arachnothera polioptera, Gad, Cat. B. IX, n. 111, pl. I, f. 1 (1884).

[246] 
(548) Melipotes gymnops, Schat.

Euthyrhynchus gymnops, Gad., Cat. B. IX, p. 288 (1884).

Melipntes gymnops, Salvad., Ibis, 1884, p. 328. - Guillem., P. Z. S. 1885, p. 641 (Arfak).

\section{Sp. 1115 (548 bis) Melipotes fumigatus, MEXER (?).}

Melipotes fumigatus, Meyer, Zeitschr. f. ges, Orn. III, p. 22, Taf. IV, f. 1 (1886!

Melipotes II. gymnopi, Sclat. similis, sed pectore grisescente, abdomine rufescente et maculis cordiformibus mullis, subalaribus pallidioribus, grisco-cinnamomeis, distinguendus.

Long. tot. $0^{\mathrm{m}}, 200$; al. $0^{\mathrm{m}}, 105-0^{\mathrm{m}}, 113$; caud. $0^{\mathrm{m}}, 100-0^{\mathrm{m}}, 104$; rostri $0^{\mathrm{m}}, 018-$ $0^{\mathrm{m}}, 019$; tarsi $0^{\mathrm{m}}, 028-0^{\mathrm{m}}, 030$.

Hab. in Papuasia - Nova Guinea meridionali-orientali, Montibus Hufeisen (Hunstein).

Il Meyer alle differenze sopraindicate aggiunge che la testa è più nera e che il colore castagno-grigio del groppone del $M$. gymnops è appena indicato nella nuova specie; ma lo stesso Meyer fa notare che un esemplare della medesima provenienza ha le parti inferiori di color grigio-bruno, la gola bianco-grigia, la testa non nera ed il groppone di color castagno-grigio!

\section{(549) IMelidectes torquatus, ScLAT.}

Melidectes torquatus, Gad., Cat. B. IX, p. 285 (partim) (1884). - Guillem., P. Z. S. 1885, p. 641 (Arfak).

\section{Sp. 1116 (549 bis) Melidectes emilii, METER.}

Melidectes torquatus, Sharpe (nec Scl,), Journ. Linn. Soc., Zool. XVI, p. 438 (Morocco) (1883). - Gad., Cat. B. IX, p. 285 (partim) (1884).

Melidectes Emilii, Meyer, Zeitschr, f. ges, Orn. III, p. 22, Taf. IV, f. 2 (1886). - Sharpe, Nature, vol, 34, p. 340 (1886). - Id., Gould's B. New Guin. pt. XXiV, pl. 10 (1888).

MIelidectes M. torquato, Sclat. similis, sed corpore subtus pallide cinnamomeo et gulae macula alba minori distinguendus.

Long. tot. $0^{\mathrm{m}}, 195-0^{\mathrm{m}}, 210$; al. $0^{\mathrm{m}}, 105-0^{\mathrm{m}}, 115$; caud. $0^{\mathrm{m}}, 095-0^{\mathrm{m}}, 105$; rostri $0^{\mathrm{m}}, 022-0^{\mathrm{m}}, 025$; tarsi $0^{\mathrm{m}}, 024-0^{\mathrm{m}}, 027$.

$H a b$. in Papuasia - in Nova Guinea meridionali-orientali, Montibus Astrolabii (Goldie), Sogeri (Forbes), Montibus Hufeisen (Hunstein).

Il Mejer fa notare che le piume chiare delle parti inferiori, dei lati del collo e del petto sono di color cannella chiaro, e non bianche, come nel $M$. torquatus, nel quale si nota soltanto una tinta cinnamomea sotto la fascia nera del petto; inoltre la fascia bianca della gola è stretta e trasversale, non larga e longitudinale; il nero della testa si estende più in addietro e passa gradatamente nel bruno-scuro della cervice; le cuopritrici inferiori delle ali sono di color cinnamomeo-chiaro e non isabella; l'area nuda alla base delle mandibole è più estesa; le dimensioni non sono molto diverse. Questa è la forma meridionale del genere Melidectes. 
(550) Melirrhophetes leucostephes, MeYrr.

Melirrhophetes leucostephes, Gad., Cat. B. IX, p. 289 (1884) - Guillem., P. Z. S. 1885, p. 641 (Arfak).

(551) Melirrhophetes ochromelas, MEYER.

Melirrhophetes ochromelas, Gad., Cat. B. IX, p. 289 (1884).

Sp. 1117 (551 bis) Melirrhophetes batesi, SHarPe (?).

Melirrhophetes batesi, Sharpe, Nature, vol. 34, p. 340 (Aug. 12, 1886)。-Id., Gould's B. New Guin. pt. XXII, pl. 10 (1886). - Ibis, 1889, p. 583.

MI. similis M. ochromelani, Meyer, sed fascia supraoculari cervina distinguendus.

Long. tot. $0^{\mathrm{m}}, 228$; al. $0^{\mathrm{m}}, 122$; caud. $0^{\mathrm{m}}, 104$; rostri $0^{\mathrm{m}}, 033$; tarsi $0^{\mathrm{m}}, 026$.

$H a b$. in Papuasia - Nova Guinea meridionali-occidentali, regione Sogeri dicta (Forbes) (Sharpe).

Io dubito che questa specie non sia veramente distinta dal $I I$. ochromelas, giacchè lo Sharpe non ha potuto confrontare gli esemplari della Nuova Guinea meridionale-orientale con altri del $M$, ochromelas, ma soltanto colla figura di questa specie data dal Gould (B. of New Guin. pt. IV, pl. 6), la quale è molto inesatta, non avendo ben disegnate le due macchie rossigne, una sopra lo spazio nudo postoculare, e l'altra dietro l'estremità delle piume auriculari superiori, le quali io ho esattamente descritte nella mia Ornitologia, vol. II, p. 321 ; inoltre in quella figura le piume auriculari sono circondate posteriormente da una fascia bruna, che in realta non esiste e che dotrebbe essere limitata all'estremità delle piume auricolari superiori, e siccome appunto per la presenza di quelle due macchie rossigne e per la mancanza della fascia bruna postauricolare lo Sharpe ha creduto di distinguere gli esemplari della Nuova Guinea meridionale-orientale, si può conchiudere che questi non differiscono da quelli della Nuova Guinea settentrionale-occidentale, almeno per quei caratteri.

\section{(552) Meliarchus sclateri, Grar.}

Phílemon sclateri, Ramsay, Pr. Linn. Soc. N. S. W. VII, p. 26 (1882) (Isole Salomone). - Gad., Cat. B. Brit. Mus. IX, p. 279 (1884). - Salvad., Ibis, 1884, p. 328.

Meliarchus sclateri, Sharpe, Gould's B. New Guin. pt. XXI, pl. 5 (1886).

Comunissimo nelle Isole Salomone; frequenta i fiori della Palma del Coco.

(553) Stigmatops ocularis (GooLD).

Glyoyplila ocularis, part., Gad., Cat. B. Brit. Nus. IX, p. 213 (1884).

(554) Stigmatops albo-auricularis, RAMSAY.

Glycyphila albo-auricularis, Gad., Cat. B. Brit. Mus. IX, p. 217 (1884).

Stigmatops albo-auricularis, Sharpe, Gould's B. New Guin. pt. XVII, pl. 13 (1884). - Ramsay, Tab. List, p. 12 (1888).

(555) Stigmatops argentauris (FINSCH).

Glycyphila argentauris, Gad, Cat. B. IX, p. 215 (1884).

[248] 
(556) Stigmatops chloris, Salvad.

Stigmatops chloris, Salvad., Ibis, 1884, p. 327. - Sharpe, Rep. Voy. H. M. S. Alert, Birds, p. 19 (1884)。 - Id., Gould's B. New Guin. pt. XX, pl. 23 (1885).

Glycyphila ocularis, part., Gad., Gat, B. Brit. Mus. IX, p. 213 (1884).

(557) Stigmatops squamata, SALVAD.

Glycyphila squamata, part., Gad., Cat. B. Brit. Nus, IX, p. 213 (1884)。 - Sharpe, Gould's B. New Guin. pt. XXI, pl. 7 (1886). - Büttik., Not. Leyd. Nlus, VIII, p. 64 (partim) (1886).

\section{Sp. 1118 (557 bis) Stigmatops salvadorii, MeYer.}

Nectarinia, sp. inc., Sclat., P. Z. S. 1883, p. 51 (Loetoe and Larat).

Stigmatops squamata, Sclat. (nec Salvad.), P. Z. S. 1883, pp. 195, 198. - Forbes, P. Z. S. 1884, p. 432. - Salvãd., Ibis, 1884, p. 355. - Sharpe, Gould's B. New Guin. pt. XXI, pl. 7 (partim) (1886). - Büttik., Not. Leyd. Mus, VIII, p. 64 (partim) (1886).

Glycyphila squamata, Gad., Cat. B. Brit. Mus, IX, p. 217 (partim) (1884).

Stigmatops Salvadorii, Meyer, Zeitschr. f. ges. Orn. 1884, p. 217 (Timorlaut) (Tipo esaminato).

Stigmatops St. squamatae, Salvad. simillima, sed supra minus olivascens et magis grisescens et subtus minus flavicans, et magis albicans.

Long. tot. circa $0^{\mathrm{m}}, 140$; al. $0^{\mathrm{nn}}, 070-0^{\mathrm{m}}, 062$; caud. $0^{\mathrm{m}}, 056-0^{\mathrm{m}}, 050$; rostri $0^{\mathrm{m}}, 018-0^{\mathrm{m}}, 015 ;$ tarsi $0^{\mathrm{m}}, 021-0^{\mathrm{m}}, 017$.

Hab, in Timor-laut (Forbes, Riedel).

Nè lo Sharpe, nè il Büttikofer ammettono che gli esemplari di Timor-laut siano specificamente distinti da quelli di Khhoor, alla quale opinione non saprei acconciarmi dopo l'esame da me fatto di sei esemplari tipici della St. salvadorii, tra i quali due considerati dal Neyer come costituenti una varietà per avere le parti superiori più grigie e meno olivastre, e le inferiori più bianchiccie e meno giallognole degli altri quattro esemplari. Se io non m'inganno, questi due esemplari sono adulti e gli altri sono esemplari più o meno giovani; e quindi sarebbero i giovani, più olivacei superiormente e più giallognoli inferiormente, che somigliano alla S. squamata, di Khoor, laddove gli adulti ne sono ben distinti.

\section{Sp. 1119 (557ter) Stigmatops kebirensis, MEYER.}

Stigmatops kebirensis, Meyer, Zeitschr. f. ges. Orn. I, p. 218 (1884) (Babbar) (Tipi esaminati). - Sharpe, Gould's B. New Guin. pt. XXl, pl. 6 (1886).

Griseo-olivacea, uropygio vividiori; subtus favescens, pectore summo squamato, sed pectoris plumis griseo-olivaceis, late flavo-albescenti limbatis; subalaribus albescentibus; regione oculari genisque nigro et albo squamatis; auricularibus griseoargenteis; fascia mandibulari grisco-cinerea; alis rectricibusque fusco-griseis, olivaceo marginatis; rostro nigro; pedibus plumbeis.

Long. tot. $0^{\mathrm{m}}, 136-0^{\mathrm{m}}, 137$; al. $0^{\mathrm{m}}, 073-0^{\mathrm{m}}, 074$; caud. $0^{\mathrm{m}}, 061-0^{\mathrm{m}}, 062$; rostri $0^{\mathrm{m}}, 017-0^{\mathrm{m}}, 018 ; \operatorname{tarsi} 0^{\mathrm{m}}, 019-0^{\mathrm{m}}, 021$.

$H a b$, in Papuasia - Ins. Babbar seu Kebir (Riedel).

Ho esaminato due esemplari tipici di questa specie, la quale si distingue dalla $S$. salvadorii non pel colorito più grigio delle parti superiori, ma pel disegno a squame 
delle parti inferiori meno esteso, essendo esso confinato alla parte inferiore del collo e superiore del petto; inoltre le macchie scure sul mezzo delle piume del petto sono più acute e la macchia grigia alla base della mandibola inferiore più cospicua.

\section{(1007) Stigmatops blasii, SalvaD}

Myzomela n. sp., Meyer, Sitzb. u. Abh. Gesellsch. Isis, 1884, Abh. I, p. 40 (1884) (Ceram). - Id., Zeitschr. f. ges. Orn. I, p. 208 (1884).

Stigmatops blasii, Salvad., Ibis. 1884, p. 208. - Neyer, Zeitschr. f. ges. Urn. I, p. 208 (1884).

Supra grisea, viridi tincta; pilei plumis linea fusca scapali notatis; supracaudalibus griseis; alis coudaque obscure griseo-fuscis; secundariis in pogonio externo olivaceo, primariis albido limbatis; subtus grisca; pectore vix virescenti lavato; gula squamata, plumis in medio infuscatis et pallide griseo marginatis; subalaribus atbidis.

Long. tot. circa $0^{\text {ma }}, 110$; al. $0^{\mathrm{m}}, 055$; caud. $0^{\mathrm{m}}, 045$; rostri $0^{\mathrm{m}}, 015$; tarsi $0^{\mathrm{m}}, 016$.

Hab. in Moluccis - Ceram (Riedel).

Io avera espresso il dubbio che l'uccello descritto dal Meyer fosse identico colla Stigmatops blasii, Salvad., e sebbene il Meyer, dopo aver confrontato i suoi esemplari col tipo della mia specie, dichiari che essi non sono identici, tuttavia per l'esame fatto dei due esemplari di Ceram menzionati dal Meyer, a me non riesce di trovare sufficienti caratteri per doverli separare specificamente.

Il Meyer ha creduto di dover considerare i suoi esemplari come spettanti ad una specie di Myzomela anzichè ad una Stigmatops, ma il disegno a squame che si scorge sulla gola mi fa inclinare a credere che si tratti invece di una vera Stigmatops. La St. blasii è stata ommessa dal Gadow nel Catalogue of the Birds in the British Museum, vol. IX.

\section{(558) Ptilotis analoga, RCHB.}

Ptilotis analoga, Sharpe, Journ. Linn. Soc., Zool. XVI, p. 438 (Norocco) (1882). - Gad., Cat. B. IX, p. 227 (partim) (1884). - Neyer. Zeitschr. f. fes. Orn. I, p. 289 (1884. - Nehrk., Journ. f. Orn. 1885, p. 33 (Waigeu) (uovo). - Guillem., P. 7. S. 1885, p. 641 (Waigiou).

Ptilotis similis?, Meyer, Sitzb. u. Abh. Gesellsch. Isis, 1884, Abb. 1, p. 41 (Nido).

Ptilotis gracilis, Ramsay, Tah. List, p. 12 (1888).

Ptilotis crhysotis, Forbes (nec. Auct.), P. Z. S. 1888, p. 124 (Cape York). - Id., Voy. Chall. Birds., p. 88 (1881) ( =P. nolata, Gould, lide Sharpe, Report Alert, p. 20).

Ptilotis aruensis, Sharpe, Heport Alert, p. 19 (1884).

? Ptilotis simplez, Bernst, in Musschenbr, Dagboek, p. 144 (Sorong) (1883/.

Nella Ornitologia, dopo aver annoverato quasi 70 esemplari che tutti riferii alla $P$. analoga, io feci notare come essi potessero essere attribuiti a tre forme diverse; ma intorno al valore delle medesime io non osai pronunciarmi, non trovando quelle tre forme circoscritte in differenti aree, tranne una, quella delle Isole Aru e della Nuova Guinea meridionale, che lo Sharpe ha creduto di dover distinguere specificamente col nome di $P$. aruensis.

Credo opportuno di esporre qui le idee dello Sharpe intorno alla Ptitotis ana$\log a$, Rchb., quale io l'ho ammessa:

Lo Sharpe vorrebbe riconoscere nella medesima due principali forme:

1. Una con lungo ciuffo auricolare giallo, e colle piume del groppone copiose $[250]$ 
e molli e con sfumature bruno-nericcie subapicali, e quelle più esterne o laterali con macchie bianche all'apice. A questa forma (Pt. analoga) apparterrebbero gli esemplari di Dorei, Mysol e Waigiou.

2. Gli esemplari di tutte le altre località avrebbero le piume auricolari formanti un ciuffo arrotondato (e più breve) e questi apparterrebbero a due forme:

a) $P$. aruensis, Sharpe, delle Isole Aru, col groppone variegato, come negli esemplari della Nuova Guinea settentrionale-occidentale.

b) Pt. notata, colle piume del groppone non variegate; e queste alla sua volta si suddividerebbero in due forme; una più grande ( $P$. notata, Gould) ed una più piccola ( $P$. gracilis, Gould) della Nuova Guinea orientale-meridionale e del capo York.

In conclusione lo Sharpe riconoscerebbe quattro forme, le quali si potrebbero distinguere ai seguenti caratteri :

a. plumis auricularibus flavis elongatis; plumis uropygii mollibus copiosis, nigro-fusco variegatis, exterioribus albo terminatis

b. plumis auricularibus flavis breviusculis:

1. P. analoga, Rchb. (ex Dorei, Mysol, Waigiou).

$a^{\prime}$. plumis uropygii uti in $P$. analoga . . . . . . 2. $P$. aruensis, Sharpe(ex Ins. Aru).

$b$. plumis uropygii haud variegatis:

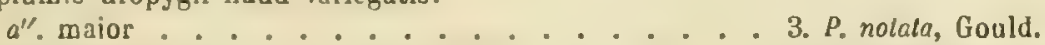

l". minor . . . . . . . . . . 4. P. gracilis, Gould (ex Nova Hollandia septentrionali et Nova Guinea meridionali-orientali).

Lo Sharpe non indica i limiti dell'area nella quale si troverebbe la $P$. notata. A queste conclusioni dello Sharpe non saprei ancora acconciarmi, giacchè le quattro forme non hanno realmente una distribuzione geografica quale egli afferma.

\section{(559) Ptilotis flavirictus, Salvad.}

Ptilotis analoga, part., Gad., Cat. B. IX, p. 227 (1884).

Ptilotis flavirictus, Salvad., Ibis, 1884, p. 327.

(560) Ptilotis albonotata, Salvad.

Ptilotis albonotata, part, Gad, Cat. B. Brit. Mus. IX, p. 229 (1884), - Salvad., Ibis, 1884, p. 327. - Sharpe, Gould's B. New Guin. pt. XX, pl. 5 (1885).

(561) Ptilotis montana, SAluVAD.

Ptilotis albonotata, part., Gad. Cat. B. IX, p. 229.

Ptilotis montana, Salvad, Ibis, 1884, p. 327.

Il Gadow, senza aver veduto un esemplare di questa specie, ha creduto di doverla riferire alla $P$. albonotata, dalla quale io sostengo essere diversa.

\section{(562) Ptilotis versicolor, Gould.}

Ptilotis versicolor, Gad., Cat. B. IX, p. 234 (1884). - Finsch u. Meyer, Zeitschr. f. ges. Orn. 1886, p. 23 (Aroani, presso Milne Bay). - Ramsay, Tab. List, p. 12 (1888).

(563) Ptilotis sonoroides, G. R. GR.

Ptilotis sonoroides, Gad., Cat. B. IX, p. 235 (1884). - Nehrk., Journ. f. Orn. 1885, p. 33 (Waigeu). - Guillem., P. Z. S. 1885, p. 641 (Mysol. - Meyer, Zeitschr. f. ges. Orn. 1886, p. 35 (Jamma). 
(564) Ptilotis germana, RAMSAX.

Ptilotis germana, Gad., Cat. B. IX, N. 246 (1884). - Ratnsay, Tab. List, p. 13 (1888).

Ptilotis flavescens, part., Ramsay, l. c. p. 378 (1888).

Hab. in ins. freti Torresii (Ramsay); in Nova Hollandia, ad caput York (Ramsay).

Suppongo che il Ramsay abbia per errore indicata la Nuova Guinea meridionale fra i luoghi abitati dalla $P$. flavescens, poichè questa è rappresentata in quella regione dalla $P$. germana.

(565) Ptilotis subfrenata, Salvad.

Ptilotis subfrenata, Gad., Cat. B. IX, p. 231 (1884.

(566) Ptilotis erythropleura, Salvad.

Ptilotis erythropleura, Gad, Cat. B. IX, p. 249 (1884).

(567) Ptilotis cinerea, Sclat.

Ptilotis cinerea, Gad., Cat. B. IX, p. 247 (1887). - Guillem., P. Z. S. 1885, p. 642 (Arfak).

\section{Sp: 1120 (567 bis) Ptilotis fulvo-cinerea, Merer.}

? Stomiopera unicclor, Ramsay (nec Gould), Pr. Linn. Soc. N. S: W. II, p. 483, n. 363 (1878)

(S. Coast N. Guinea), - Salvad., Orn. II, p. 326 (1880). - Ramsay, Tab. List, p. 13 (1888).

Ptilotis fulvo-cinerea, Meyer, Zeitschr. f. ges. Orn. III, p. 24, Taf. V, f. 1 (1886).

Ptilotis Pt. unicolori, Gould, simitis, sed major, sine colore olivacco capitis et cervicis plumis fuscis, cinereo marginatis et remigum marginibus internis subtus albo-griseis. $0^{\mathrm{m}}, 024$.

Long. tot. $0^{\mathrm{m}}, 215$; al. $0^{\mathrm{m}}, 115$; caud. $0^{\mathrm{m}}, 100$; rostri $0^{\mathrm{m}}, 028$; tarsi $0^{\mathrm{m}}, 023-$

Hab. in Nova Guinea meridionali-orientali, in Montibus Hufeisen (Hunstein).

Secondo il Meyer, sarebbe questa una grande specie facile da distinguere, di color grigio leggermente rossigno (che nella figura non appare punto), le piume della testa e del collo hanno strie longitudinali-nericcie, il dorso è meno striato; le parti inferiori e le cuopritrici inferiori delle ali sono di color grigio-cinereo uniforme un poco più chiaro, le ali e la coda di color cinereo-bruno, i margini esterni delle remiganti alquanto più grigi e gli interni inferiormente grigio-bianchicci.

Inoltre questa specie, sempre secondo il Meyer, differisce dalla Pt. cinerea, Sclat. per la testa grigio-rossigna e per le dimensioni maggiori del becco, e dalla P.marnorata per essere questa tutta variegata.

亡̀ probabile che questa specie sia stata confusa dal Ramsay colla Stomiopera unicolor.

(1008) Ptilotis marmorata, SHARPE.

Ptilotis marmorata, Sharpe, Gould's B. New Guin. pt. XIV, pl. 9 (1883). - Gad., Cat. B. IX, p. 247, pl. IV (1884). - Salvad., Ibis, 1884, p. 329. - Meyer, Zeitschr. f. ges. Orn. III, p. 24 (1886).

\section{(568) Ptilotis ixoides, Salvad.}

Ptilotis ixoides, Gad, Cat. B. IX; p. 222 (nota) (1884) 
(569) Pycnopygius stictocephalus (Salvad.).

Pycnopygius stictocephalus, Gad., Cat. B. IX, p. 290 (1884).

(570) Euthyrhynchus griseigula, ScHLEG.

Euthyrhynchus griseigularis, Gad, Cat. B. Brit. Mus. IX, p. 287 (1884).

Euthyrhynchus griseigula, Sharpe, Gould's B. New Guin. pt. XX, pl. 9 (1885).

(571) Euthyrhynchus flavigula, Schleg.

Euthyrhynchus flavigularis, Gad., Cat, B. Brit. Mus. IX, p. 287 (1884).

Euthyrhynchus griseigularis, Guillem. (nec Schleg.), P. Z. S. 1885, p. 642.

Euthyrhynchus flavigula, Sharpe, Gould's B. New Guin. pt. XX, pl. 7 (1885).

(572) Euthyrhynchus fulvigula, ScBLEG.

Euthyrhynchus fulvigularis, Gad., (lat. B. Brit. Mus. IX, p. 287 (1884).

Euthyrhynchus fulvigula, Sharpe, Gould's B. New Guin. pt. XX, pl. 8(1885). - ? Mever, Zeitschr. f. ges. Orn. III, p. 25 (1886).

? Euthyrhyncha fulvigula, Meyer, l. c. p. 181 (1886).

Hab. in Nova Guinea meridionali-orientali, Montibus Hufeisen (Hunstein).

Il Meyer accenna ad alcune lievi differenze fra gli esemplari della Nuova Guinea orientale-meridionale e quelli dei Monti Arfak.

\section{(1009) Euthyrhynchus fulviventris (RAMSAY).}

Euthyrhynchus fulviventris, Gad., Lat. B. Brit. Mus. IX, p. 288 (1884). - Sharpe, Gould's B. New Guin. pt. XX (nel testo dell'E. griseigula).

Questa specie è stata riconosciuta come distinta per le parti superiori di color bruno-olivaceo e per le inferiori di color bruno-vinaceo con leggera tinta giallo-olivacea sulla gola.

(573) Xanthotis polygramma (G. R. GR.).

Ptilotis polygramma, Gad., Cat. B. IX, p. 233, pl. VI (1884)。

Xanthotis polygramma, Meyer, Zeitschr. f. ges. Orn. IlI, p. 24 (1886) (Monti Hufeisen).

Ho esaminato uno degli esemplari dei Monti Hufeisen nella Nuova Guinea meridionale-orientale, che secondo il Meyer sembrano differenti da quelli della Nuova Guinea settentrionale-occidentale pel nero delle macchie del petto più cupo e per le timoniere con margini più vivi, e non ho potuto scorgere differenze apprezzabili, confrontandolo con altri del Wa-Samson e del Fiume Fly.

\section{(574) Xanthotis fligera (GooLD).}

Xanthotis filigera, Salvad., Voy. Chall., Birds, p. 80 (1881). - Sharpe, Journ. Linn. Soc., Zool. XVI, p. 440 (Morocco) (1882). - Meyer, Zeitschr. f. ges. Orn. I, p. 289 (1884).

Ptilotis Aligera, Gad., Cat. B. IX, p. 237 (1884). - Finsch, Vög. d. Südsee, p. 33 (1884).

(575) Xanthotis chrysotis (LESS.).

Ptilotis chrysotis, part., Gad, Cat. B. Brit. Mus. I, p. 238 (1884). - Guillem., P. Z. S. 1885, p. 642. Xanthotis chrysotis, Salvad., Ibis, 1884, p. 327. - Sharpe, Guuld's B. New Guin. pt. XXI, pl.8(1886). Xanthotis rubiensis, Meyer, Zeitschr. f, ges. Orn. I, p. 289 (1884).

Ho esaminato due esemplari tipici della $X$. rubiensis, Mejer, e non sono riuscito

17 T. Salvadori. 
a trovarvi traccie di quelle macchie nucali grigie, che il Meyer descrive e per le quali dovrebbe somigliare alla $X$. fitigera, Gould, quindi credo che abbia ragione lo Sharpe, il quale ha riunito la $X$. rubiensis alla $X$. chrysotis (Less.).

\section{(576) Xanthotis fusciventris, SALVAD.}

Ptilotis chrysotis, part., Gad., Cat. B. IX, p. 238, specim. $d$ (1884).

Ptilotis fusciventris, Gad., Cat. B. IX, p. 239 (1884). - Guillem., P. Z. S. 1885, p. 642 (Waigiou). Xanthotis fusciventris, Salvad., Ibis, 1884, p. 327.

(577) Xanthotis meyeri, SALVAD.

Ptilotis meyeri, Gad., Cat. B. IX, p. 239 (1884). - Meyer, Zeitschr. f. ges, Orn. 1886, p. 35 (Kafu).

(578) Melitograis gilolensis (Temm.).

Melitograis gilolensis, Gad., Gat. B. IX, p. 282 (1884). - Guillem., P. Z. S. 1885, p. 572 (Batchian).

(579) Philemonopsis meyeri, Salvad.

Philemon meyeri, Gad., Cat. B. IX, p. 281 (1884).

(580) Philemon moluccensis (GM.).

Philemon moluccensis, part., Gad., Cat. B. IX, p. 275 (1884. - Salvad., Ibis, 1884, p. 328.

Il Gadow ha erroneamente riferito a questa specie gli esemplari delle isole Kei.

(581) Philemon plumigenis (G. R. GR.).

Philemon plumigenis, Salvad., Voy. Chall. Birds, p. 70 (1881). - Meyer, Verh. z.-b. Gesellsch. Wien, 1881, p. 773 (Kei). — Gad., Cat. B. IX, p. 279 (partim) (1884).

Sp. 1121 (581 bis) Philemon timorlaoensis, MEYER.

Philemon plumigenis, Sclat. (nec Gray), P. Z. S. 1883, pp. 51, 195, 200. - Gad., Cat. B. IX, p. 279 (partim) (1884). - Sharpe, Gould's B. New Guin. pt. XVI, pl. 13 (1884).

Philemon timorlaoensis, Meyer, Sitzb. u. Abh. Gesellsch. Isis, 1884, Abh. I, p. 41 (Timor-laut). Salvad., Ibis, 1884, pp. 328, 355. - Id., P. Z. S. 1884, p. 579.

Philemon timorlautensis, Forbes, P. Z. S. 1884, pp. 429, 432.

Supra obscure griseus, subtus pallidior, gula et collo antico clarioribus, scapis plumarum colli antici fuscis; collari cervicali clare cinereo; alis et cauda dorso concoloribus, caudae apice vix pallidiore; cute muda sircumoculari, rostro tubere destituto et pedibus nigris.

Long. tot. $0^{\mathrm{m}}, 320-0^{\mathrm{m}}, 300$; al. $0^{\mathrm{m}}, 160-0^{\mathrm{m}}, 144$; caud. $0^{\mathrm{m}}, 138-0^{\mathrm{m}}, 125$; rostri $0^{\mathrm{m}}, 045-0^{\mathrm{m}}, 038 ;$ tarsi $0^{\mathrm{m}}, 040-0^{\mathrm{m}}, 039$.

Hab. in Papuasia - Timor-laut (Forbes, Riedel).

Obs. Philemon Ph. plumigeni, Gr. simillimus, sed capite paullo pallidiore et rostro subtiliore distinguendus.

Ho esaminato tre esemplari di questa specie, la quale differisce pochissimo dal Ph. plumigenis. Io non sono riuscito a mettere d'accordo la descrizione data dal Meyer cogli esemplari esaminati, giacchè in quella le parti superiori sono descritte fulvescenti (supra fulvescens), l'apice della coda, il sottocoda, le cuopritrici inferiori 
delle ali ed il vessillo interno delle remiganti di color fulvo (!) e gli steli delle remiganti e delle timoniere più o meno rossigni !

Lo Sharpe ha riferito gli esemplari di Timorlaut al $P h$. plumigenis, tuttavia fa notare come essi differiscano alquanto da quelli delle isole Kei.

(582) Philemon fuscicapillus (W $W_{A L L}$.).

Philemon fuscicapillus, Gad., Cat. B. IX, p. 279 (1884)

(583) Philemon albitorques, SCLAT.

Philemon albitorques, Sclat., Voy. Chall., Birds, p. 28, pl. VIII (1881). - Gad., Cat. B. IX, p. 278 (1884).

11 Gadow ha ommesso di citare il viaggio del Challenger.

(584) Philemon subcorniculatus (H. et J.).

Philemon subcorniculatus, Blas, P. Z. S. 1882, p. 708 (Ceram). - Gad., Cat. B. IX, p. 280 (1884).

(585) Philemon cockerelli, ScLat.

Philemon cokerelli, Gad., Cat. B. IX, p. 278, pl. II (1884). - Finsch, Vög. d. Südsee, p. 10 (Nova Britannia) (1884).

(586) Philemon jobiensis, MEYER.

Philemon jobiensis, Gad., Cat. B. IX, p. 273 (1884). - Salvad., Ibis, 1884, p. 328. - Meyer, Sitzb. u. Ahb. Gesellsch. Isis, 1884, Abh. I, p. 12 (nido) (1884). - Id., Zeitschr. f. ges. Orn. I, p. 290 (1884), III, p. 35 (1886).

Rhectes (errore) jobiensis, Meyer, Sitzb. u. Abh. Gesellsch. Isis, 1884, Abh. I, p. 31 (uovo).

Tropidorhynchus jobiensis, Meyer, Zeitschr. f. ges. Orn. I, p. 216 (1884).

Hab. in Nova Guinea, Kafu (Laglaize).

Gli esemplari di Kafu, secondo il Meyer, differiscono per l'ala un centimetro più breve, pel colore chicro della gola più esteso in basso e per tutto il colorito più grigio.

Non so intendere perchè il Meyer vorrebbe riferire questa specie, che è priva di tuberosità alla base del culmine del becco, al genere Tropidorhynchus, anzichè al genere Philemon, che comprende le specie senza tuberosità.

(587) Tropidorhynchus novae guineae, S. MülL.

Tropidorhynchus cornutus, Bernst., in Musschenbr., Dagboek, pp. 151, 154 (Sailolo) (1883). Philemon novae guineae, Gad., Cat. B. IX, p. 274 (1884).

Tropidorhynchus novae guineae, Finsch. Vög. d. Südsee, p. 26 (Port Moresby) (1884). - Nehrk., Journ. f. Orn. 1885, p. 33 (Waigeu). - Guillem., P. Z. S. 1885, p. 643 (Aru, Waigiou, Batanta). Tropidorhynchus aruensis, Meyer, Zeitschr. f. ges. Orn. I, pp. 216, 290 (Aru) (1884).

Il Meyer ha creduto di poter separare specificamente gli esemplari dello isole Aru, per certe piccole differenze che anche io areva notate, cioè per le piume della testa che si avanzano più verso la fronte, pel colorito più chiaro e pel tubercolo frontale piừ grande.

Ad onta di tutto ciò io non credo che gli esemplari delle Isole Aru si possano separare specificamente da quelli della Nuova Guinea, sebbene il Meyer, ad avralorare la differenza, affermi che i primi somiglino più al Tr. timoriensis, Müll. che non al Tr. novae guineae. 


\section{(588) Melithreptus albigularis, GooLD.}

Melithreptus albigularis, Gad., Cat. B. IX, p. 265 (1884). - Ramsay, Tab. List, p. 14 (1888).

(589) Zosterops fusca, Bernst.

Zosterops fusca, Sharpe, Cat. B. IX, p. 146 (nota) (1884).

(590) Zosterops atriceps, G. R. GR.

Zosterops atriceps, Sharpe, Cat. B. IX, p. 200 (1884).

(591) Zosterops fuscifrons, SALvad.

Zosterops fuscifrons, Sharpe, Cat. B. Brit. Mus. IX, p. 201 (1884). - Id., Gould's B. New Guin. pt. XVIII, pl. 13 (1884).

(592) Zosterops mysoriensis, MEYeR.

Zosterops mysoriensis, Sharpe, Cat. B. IX, p. 201 (1884).

(593) Zosterops hypoleuca, SaLvad.

Zosterops hypoleuca, Sharpe, Cat. B. IX, p. 202 (1884).

(594) Zosterops grayi, WALL.

Zosterops grayi, Sharpe, Cat. B. IX, p. 162 (1884).

(595) Zosterops albiventris, RCHB.

Zosterops flavogularis, Ramsay, Pr. Linn. Soc. N. S. W. II, p. 177 (1877).

Zosterops albiventer, Sharpe, Cat. B. IX, p. 164 (1884). - Id., Report Alert, p. 17 (1884).

Sp. 1122 (595 bis) Zosterops crissalis, SHARPE.

Zosterops crisisalis, Sharpe; Cat. B. Brit. Mus. VIII, p. 165 (1884).

Zosterops Z. albiventri similis, sed minor, supra viridi-flavescens, gula laete flava, subcaudalibusque flavo-aureis distinguenda.

Long. tot. $0^{\mathrm{m}}, 101$; al. $0^{\mathrm{m}}, 56$; caud. $0^{\mathrm{m}}, 040$; rostri $0^{\mathrm{m}}, 012$; tarsi $0^{\mathrm{m}}, 015$.

Hab. in Papuasia - in Nova Guinea meridionali-orientali, Montibus Astrolabii (Goldie).

(596) Zosterops novae guineae, SALVAD.

Zosterops novae guineae, Sharpe, Cat. B. IX, p. 175 (1884). - Meyer, Zeitschr, f. ges. Orn. I, p. 291 1884). - Guillem., P. Z. S. 1885, p. 643 (Arfak).

Lo Sharpe menziona Amboina e Ceram fra le località abitate da questa specie, ma io feci già notare che quelle località, asserite per esemplari raccolti ed inviati dallo Hoedt e dal Moens, sono molto probabilmente erronee.

\section{Sp. 1123 (596 bis) Zosterops griseiventris, ScLaT.}

Zosterops griseiventris, Sclat, P. Z. S. 1883, pp. 195, 199. - Meyer, Sitzb. u. Abh. Gesellsch. Isis, 1884, Abh. I, p. 42 (Babbar) - Id., Zeitschr. f. ges. Orn. 1, p. 194 (1884). - Forbes, P. Z. S. 1884, p. 432 .

Zosterops griseiventer, Sharpe, Cat. B. IX, p. 174 (1884).

Supra laete viridis, annulo periophthalmico distincto albo; alis caudaque nigri- 
cantibus, viridi limbatis; subtus pallide grisea, in ventre medio albicantior; gula et crisso flavis; subalaribus et remigum marginibus interius albis, campterio flavido; rostro pallide corneo; pedibus pallide fuscis (Sclater); iride brunnea.

Long. tot. $0^{\mathrm{m}}, 115$; al. $0^{\mathrm{m}}, 058$; caud. $0^{\mathrm{m}}, 044$; rostri $0^{\mathrm{m}}, 016$; tarsi $0^{\mathrm{m}}, 019$.

Hab. in Papuasia - Ins. Tenimberensibus (Forbes); Babbar (?) (Riedel).

Questa specie sembra affine alla $Z$. novae guineae, Salvad.

Lo Sharpe fa notare che alla medesima sono stati riferiti esemplari di Moloe e Loetoe, ed una femmina di Larat, la quale differisce dai maschi per essere piú. grande (lung. tot. $0^{\mathrm{m}}, 119 ;$ al. $0^{\mathrm{m}}, 063$; tarso $0^{\mathrm{m}}, 020$ ) e per avere i fianchi più decisamente grigi e senza la tinta isabellina che distingue $i$ maschi; non è quindi improbabile che la detta femmina appartenga ad una forma distinta.

Il Meyer scrive che anche un esemplare di Babbar differisce alquanto da un altro di Timorlaut.

(597) Zosterops aureigula, SALVAD.

Zosterops aureigula, Sharpe, Cat. B. IX, p. 176 (1884).

(598) Zosterops chrysolaema, SALVAD.

Zosterops chrysolaema, Sharpe, Cat. B. IX, p. 177 (1884)

(599) Zosterops sp. (?)

Zosterops frontalis, Salvad. (nec Richb), Ann. Mus. Civ. Gen. XII, p. 342 (1878).

? Zosterops delicatula, Sharpe (partim). - Id., Cat. B. IX, p. 177 (1884).

Lo Sharpe ha fatto notare come il nome $Z$. frontalis fosse già stato adoperato dal Reichenbach; io mi astengo dal sostituire a quello un altro nome, eśsendo probabile, come crede lo Sharpe, che la mia $Z$. frontalis sia identica colla sua $Z$. delicatula, col quale nome in quel caso la specie dorrà essere distinta.

(1010) Zosterops delicatula, SHARPE.

Zosterops delicatula, Sharpe, Gould's B. New Guin. pt. XIV, pl. 13 (1883).

È cosa probabile, ma non ancora certa, che questa specie sia identica colla mia $Z$. fronta7is, colla quale dorrà essere confrontata; lo Sharpe da prima per distinguerla si fondò sulla tinta grigia dei lati del petto, che sarebbe caratteristica della sua specie.

(600) Zosterops intermedia, WALL.

Zosterops intermedia, Sharpe, Cat. B. IX, p. 185 (1884).

(601) Zosterops chloris, S. MüLL.

Zosterops chloris, Salvad, Voy. Chall., Birds, p. 66 (1881). - Sharpe, Cat. B. IX, p. 184 (1884).

(602) Zosterops buruensis, SalvaD.

Zosterops buruensis, Sharpe, Cạt. B. IX, p. 184 (1884).

(603) Zosterops griseotincta, G. R. GR.

Zosterops griseotincta, Sharpe, Cat. B. IX, p. 189 (1884). 
(604) Zosterops longirostris, RAMSAY.

Zosterops longirostris, Sharpe, Cat. B. Brit. Mus. IX, p. 189 (1884). - ld., Gould's B. New Guin. pt. XVII, pl. 12 (1884).

(605) Zosterops fuscicapilla, SALPAD.

Zosterops fuscicapilla, Sharpe, Cat. B. IX, p. 178 (1884).

(1011) Zosterops hypoxantha, SALVAD.

Zosterops hypozantha, Sharpe, Cat. B. IX, p. 178 (1884).

(606) Zosterops brunneicauda, SALtad.

Zosterops brunneicauda, Sharpe, Cat. B. Brit. Nus. IX, pp. 190, 293 (1884). - Id., Gould's B. New Guin. pt. XIX, pl. 11 (1885)。 - ?Guillem., P. Z. S. 1885, p. 508 (Sumbawa).

Tanto lo Sharpe, quanto il Guillemard asseriscono che un esemplare di Sumbawa, raccolto dal Guillemard, non differisce da un esemplare tipico della $Z$. brunneicauda!

(607) Zosterops uropygialis, SaLvaD.

Zosterops uropygialis, Sharpe, Cat. B. Brit. Mus. IX, pp. 190, 293 (1884)._Id., Gould's B. New Guin. pt. XIX, pl. 12 (1885).

Lo Sharpe fa notare che un esemplare avuto dal Museo di Leida manca anche esso delle piume palpebrali bianche, e tuttavia nella figura che egli dà del medesimo il cerchio palpebrale bianco è stato rappresentato!

\section{(1012, 1013) Zosterops rendovae, Tristr.}

Tephras (Zosternps) ugiensi, Ramsay, Pr. Linn. Soc. N. S. W. VII, p. 28 (25 Jan. 1882) (Izole Salomone).

Zosterops rendovae, Ramsay, Pr. Linn. Soc. N. S. W. VII, p. 42 (1882). -- Sharpe, Cat. B. Brit. Mus. IX, p. 188 (1884), - Id., Gould's B. New Guin. pt. XXIII, pl. 9 (1887).

Questa specie appartiene al gruppo di quelle senza cerchio palpebrale bianco, come la Z. uropygialis.

(608) Criniger affinis, H. et J.

Criniger affinis, Sharpe, Cat. B. VI, p. 86 (1881). - Blas. u. Nehrk., Verh. z.-b. Ges. Wien, 1882, p. 427 (Amboina).

(609) Criniger mystacalis, WaLL.

Griniger mystacalis, Sharpe, Cat. B. VI, p. 85 (1881).

(610) Griniger chloris, Finsch.

Criniger chloris, Sharpe, Cat. B. VI, p. 85 (1881). - Guillem., P. Z. S. 1885, p. 572 (Batchian). Sharpe, Gould's B. New Guin. pt. XXIII, pl. 5 (1887).

SUBGEN. CYANOPITTA

Typus:

Cyanopitta, Gould, Mon. Pitta, pl. 8 (1880) . . . . . . Brachyurus steerei, Sharpe. Galopitta, Sclat., Cat. B. XIV, pp. 414, 419 (1888) . . . . . . Pilla maxima, M. et S

(611) Pitta maxima, Mült. et Schleg.

Leucopitta maxima, Gould, Mon. Pitt. pl. 7 (1580).

[258] 
Pitta maxima, Meyer, Sitzb. u. Abh. Gesellsch. Isis, 1884, Abh. I, p. 43 (uovo). - Pleske, Bull. Acad. Petersb. XXIX, p. 532 (Ternate!) (1884)。 - Guillem., P. Z. S. 1885, p. 572 (Halmahera). Sclat., Cat. B. XIV, p. 419 (1888).

Io trovo tanta somiglianza fra questa specie e la $P$. steerci (Sharpe) delle Filippine, da doverle considerare come appartenenti ad un medesimo gruppo.

(612) Pitta novae guineae, MüLL. et SchlgG.

Pitta novae guineae, Meyer, Zeitschr. f. Res. Orn. 1884, p. 291. - Guillem., P. Z. S. 1885̄, p. 643. -Finsch u. Meyer, Zeitschr. f. ges. Orn. 1886, p. 26 (Nonti Hufeisen). -Sclat., Cat. B. XIV, p. 440(1888)。

(613) Pitta mafoorana, Schleg.

Pitta mafoorana, Sclat, Cat. B. XIV, p. 441 (1888).

(614) Pitta rosenbergi, Schleg.

Pitta rosenbergi, Sclat., Cat. B. XIV, p. 441 1888),

(615) Pitta simillima, Gould,

Pitta strepitans, part., Sclat, Cat. B. XIV, p. 428 (1888).

Pitta strepitans subsp. simillima, Ramsay, Tab. List, p. 11 (1888).

(616) Pitta irena, Temm.

Pitta irena, Meyer, Zeitschr. f. ges. Orn. 1884, p. 210. - Sclat., Cat. B. XIV, p. 427 (1888).

Pitta crassirostris, Sclat,, ibid.

Lo Sclater menziona soltanto la localita Timor per la $P$. irena e le isole Sulla per la $P$. crassirostris, che io non credo specificamente diversa, ed ha ommesso le altre localita Boano, Ceram e Ternate.

(617) Pitta vigorsli, Gould.

Pitta brachyura, Meyer (nec Linn.), Sitzb. u. Ahh. Gesellsch. Isis, 1884, p. 43 (Dammar).

Pitta vigorsii, Meyer, Zeitschr. f. ges. Urn. 1884, p. 210 (Timorlaut). - Salvad, P. Z. S. 1884, p. 579

- Sclat., Cat. B. XIV, p. 426 (1888).

Hab. Dammar (Riedel), Timorlaut (Riedel).

Il Meyer fa notare come questa specie e la $P$. irena abbiano una somigliante area di distribuzione, la prima una più orientale, costituita dalle isole Banda, Dammar e Timor-laut e la seconda una più occidentale formata da Timor, Boano, Ceram, Sulla e Ternate.

(618) Pitta rubrinucha, WALL.

Pitta rubrinucha, Sclat., Cat. B. XIV, p. 435 (1888).

\section{(619) Pitta mackloti, TemM.}

Pitta strenua, Gould, MS. - Elliot, Ibis, 1876, pp. 410, 411, fig. 2.

Pitta mackloti, Elliot, Ibis, 1870, pp.410, 411, f. 1. - Finsch, Vög. d. Sïdsee, p. 11 (Nova Britannia) (1884). - Meyer, Zeitschr. f. ges. Orn. 1884, p. 291. - Nehrk, Journ. f. Orn. 1885, p. 33 (uovo). Guillem., P. Z. S. 1885, p. 643 (N. New Guin. long. $139^{\circ}$ E. Bruijn). - 9 Finsch u. Meyer, Zeitschr, f. ges. Orn. 1886, p. 26 (Monti Hufeisen). - Sclat., Cat. B. XIV, p. 436 (1888). - Ramsay, Tab. List, p. 11 (1888).

Lo Sclater fa notare giustamente come la macchia cerulea, più o meno apparente 
lungo il mezzo del pileo, sia più cospicua negli esemplari degli estremi dell'area occupata da questa specie, cioè in quelli di Mysol e della Nuora Britannia; in questi, secondo il Finsch, quella macchia si trova sempre; tuttavia il Finsch non ammette che essi debbano esșere separati specificamente; egli vuole invece considerare come specie distinta gli esemplari della Nuova Irlanda ( $P$. novae hiberniae, Ramsay)!

Io avera dimenticato di citare fra i sinonimi di questa specie la $P$. strenua, Gould, fondata sopra un esemplare d'incerta provenienza, avente il becco alquanto piu robusto dell'ordinario.

Sp. 1124 (619 bis) Pitta finschi, Ramsay.

Pitta (Erythropitta) finschi, Ramsay, Pr. Linn. Soc. N. S. W. IX, p. 864 (1884) (Astrolabe Range).

Capite toto, cervice, collo et mento obscure fusco-rubescentibus; gula nigra; fascia pectorali lata caerulea, inferius fascia nigra stricta marginata; gastraeo reliquo pulchre rubro; subcaudalibus exterioribus nigricanti-caeruleo marginatis; dorso toto, cervice ima, alis et cauda saturate caeruleis, fascia pectorali concoloribus; pogonio interno remigum nigro, alis subtus nigris; macula alba caelata prope angulum alae; remigibus primariis $3^{\mathrm{a}}, 4^{\mathrm{a}}$ et interdum $5^{\mathrm{a}}$ macula alba in medio notatis; rostro nigro; pedibus fusco-nigricantibus.

Long. tot. circa $0^{\mathrm{m}}, 152-0^{\mathrm{m}}, 177$, al. $0^{\mathrm{m}}, 101$; caud. $0^{\mathrm{m}}, 050$; rostri $0^{\mathrm{m}}, 029$; tarsi $0^{\mathrm{m}}, 040$.

Hab. in Papuasia - Nova Guinea meridionali-orientali, in Montibus Astrolabii (Ramsay).

Questa specie, secondo il Ramsay, si distinguerebbe per avere il dorso tutto di color ceruleo, laddove nella $P$. mackloti il dorso è olivaceo.

Nella stessa regione si troverebbero esemplari, che hanno la regione interscapolare olivacea e questi, secondo il Ramsay, sarebbero femmine, o individui non al tutto adulti, invece, secondo il Finsch, dorrebbero essere riferiti alla $P$. mackloti.

Lo Sclater ha ommesso di menzionare la Pitta finschi, Ramsay, nel vol. XIV del Catalogue of the Birds in the British Museum.

(620) Pitta rufiventris (HEINE).

Pitta rufiventris, Guillem., P. Z. S. 1885, p. 572 (Batchian). - Sclat., Cat. B. XIV, p. 434 (1888).

(621) Pitta cyanonota, G. R. Gr.

Pitta cyanonota, Pleske, Bull. Acad. Petersb. XXIX, p. 532 (Ternate)(1884). - Sclat., Cat. B. XIV, p. 435 (1888).

Gen. MeLLOPITTA, Stejn.

Typus :

Mellopitta, Stejn., Stand. Nat. Hist. IV, Birds, p. 466 (1885) . . . . . . P. lugubris, Rosenh. Goracopitta, Sclat., Cat. B. Brit. Mus. XIV, p. 449 (1888) . . . . . . P. lugubris, Rosenb.

(622) Mellopitta lugubris (RosenB.).

Melampitta lugubris, Gould, Mon. Pitta, pl. 10 (1880).

Mellopitta lugubris, Stejneg., Stand. Nat. Hist. IV, Birds, p. 466 (1885).

Coracopitta lugubris, Sclat., Cat. B. Brit, Mus. XIV, p. 449 (1888). 
Gen. GRATERoscells, Shatpe.

Grateroscelis, Sharpe, Cat. B. Brit. Mus. VII, pp. 507, 590 (1883) . . Myiothera murinn, Temm.

$$
(623,627) \text { Crateroscelis murina (ТЕмм.). }
$$

Grateroscelis murina, Sharpe, Cat. B. VII, p. 590 (1883).

Come io aveva sospettato, a questa specie deve essere riferita, secondo lo Sharpe, Ia Sericornis fulvipectus, Ramsay; tuttavia lo Sharpe (Journ. Linn. Soc. Zool. XVI (1882), p. 440) fa notare come un esemplare dei Monti dell'Astrolabio raccolto dal Goldie abbia le parti inferiori di colore bruno-rinaceo molto più viro che non gli esemplari della Nuova Guinea settentrionale e di Waigiou.

Quid Turdirostris major, Bernst, in Nusschenbr. Dagboek, pp. 123, 131, 132, 136, 141, 154, 155, 158, 161, 162, 163 (Surong, Kalwal, Batanta) (1883)?

Quid Turdirostris minor, Bernst., op. cit., pp. 123, 133, 134, 143, 144, 162 (Sorong, Batanta\} (1883)?

(624) Crateroscelis monacha (G. R. GR.).

Crateroscelis monacha, Sharpe, Cat. B. VII, p. 591 (1883),

Brachypteryx monacha, Meyer, Zeitschr. f. ges. Urn. I, p. 291 (1884.

(625) Sericornis beccarii, 'SALVAD.

Sericornis beccarii, Sharpe, Cat. B. VII, p. $30^{\prime}$ ) (1883), - Id, in Gould's B. New Guin. pt. XV, pl. 3 (1883).

(626) Sericornis arfakiana, SAlvad.

Sericornis arfakiana, Sharpe, Cat. B. VII, p. 300 (1883). - - Id., Gould's B. New Guin. pt. XV, pl. 4 (1883).

(628) Pomatorhinus isidori, Less.

Pomatorhinus isidorii, Sharpe, Cat. B. VII, p. 432 (1883) - Guillem., P. Z. S. 1885, p. 643 (Andai, Mysol).

Pomatorhinus Jacolori (errore), Bernst., in Nusschenbr., Daghoek, p. 73 (Sorong) (1883).

Pomatorhinus Forsteri (errore?), Bernst., in Musschenbr, Dagboek, p. 118 (Sorong) (1883).

$(629,1014)$ Eupetes castanonotus, Salvad.

Eupetes pulcher, Sharpe, Journ. Linn. Soc., Zool. XVI, pp. 319, 440 (1882). - Salvad., Orn. Pap. e Mol. III, p. 548 (1882).

Eupetes castanonotus, Sharpe, Gould's B. New Guin. pt. XIV, pl. 10 (1883). - Id., Cat. B. VII, p. $340(1883$ !.

Io avera gia sospettato della identita dell'E. pulcher, Sharpe, col mio E. castanonotus, la quale cosa è stata riconosciuta per vera anche dallo Sharpe.

(630) Eupetes caerulescens, Tемм.

Eupetes caerulescens, Sharpe, Cat. B. VII, p. 339 (1883). - Guillem., P. Z. S. 1885, p.644.

(631) Eupetes nigricrissus, Salvad.

Eupetes nigricrissus, Sharpe, Cat. B. VII, p. 339 (1883).

(632) Eupetes leucostictus, Sclat.

Eupetes leucostictus, Sharpe, Cat. B. VII, p. 342 (1883). 
(633) Eupetes incertus, SALVAD.

Eupetes incertus, Sharpo, Cat. B. VII, p. 341 (1883):

(634) Cinclosoma ajax (Tenm.).

.Eupetes (Circeloroma sic) ajax, Ramsay, Pr. Linn. Soc. I. S. W. VIII, p. 26 (1883) (uovo). Cinclosoma ajax, Sharpe, Cat, B. VlI, p. 336 (1883).

(635) Drymoedus beccarii, Salvad.

Drymoedus beccarii, Salvad., Voy. Chall., Birds, p. 80 (1881. - Sharpe, Cat. B. Brit. Ilus. VII, p. 345 (1883). - Id., Gould's B. New Guin. pt. XV, pl. 8 (1883).

(636) Ortygocichla rubiginosa, Sclat.

Ortygocichla rubiginosa, Sharpe, Cat. B. VII, p. 560 (1883).

Lo Sharpe ha ommesso la citazione di questa specie che si trova nella mia Ornitologia, vol. II, p. 679 (1881).

(1015) Oreocincla papuensis (SEEBOHM).

Geocichla heinii (part.?), Ramsay, Tah. List, p. 11 (1888).

Pare che il Ramsay abbia identificato questa specie colla 0 . heinei, Cab. dell'Australia settentrionale.

Gen. TURDULUS, Hodgs.

Typus:

Turdulus, Hodgs., in Gray's Zool. Miscell. p. 83 (1844). . . . . . . Turdus wardi, Jerd.

Sp. 1125 (1015 bie) Turdulus machiki (ForBes).

Geocichla, sp. inc., Sclat., P. Z. S. 1883, pp. 51, 56, 200.

Geocichla machiki, Forhes, P. Z. S. 1883, p. 589, pl. LII; 1884, pp. 426, 433, - Meyer, Zeitschr. f. ges. Orn. I, p. 494 (1884).

Supra brunneo-olivaceus, capite paullum in schistaceum vergente, uropygio et supracaudalibus castaneo tinctis; loris albis, auricularibus albo et fusco variis; atis brunneis, tectricibus alarum minoribus fusco-olivaceis, late albo terminatis, majoribus brunneis, albo terminatis; remigibus ultimis brunneis albido terminatis; rectricibus brunneis, exterioribus late albido terminatis; mento, gula et pectore albidofulvis, gastraco reliquo albo, lateribus maculis semilunaribus nigris notatis; axillaribus dimidio basali albis, apicali nigris; tectricibus alarum inferioribus in dimidio basali brunneis, apicali albis; pogonio interno remigum ad basin albo; maxilla fusca, mandibula flava; iride brunnca; pedibus pallide carneis.

Long. tot. $0^{\mathrm{m}}, 200-0^{\mathrm{m}}, 210$; al. $0^{\mathrm{m}}, 111-0^{\mathrm{m}}, 117$; caud. $0^{\mathrm{m}}, 080-0^{\mathrm{m}}, 085$; rostri $0^{\mathrm{m}}, 020-0^{\mathrm{m}}, 026$; tarsi $0^{\mathrm{m}}, 031-0^{\mathrm{m}}, 035$.

Hab. in Papuasia - Timor-laut (Forbes).

Dice il Forbes che questa specie è intermedia al T. rubiginosus di Timor ed al T. erythronotus di Celebes.

L'esemplare menzionato dallo Sclater (l.c.) era un giovane di questa specie.

Sp. $1126\left(1015^{\text {ter })}\right.$ Turdulus schistaceus (MEYER).

Geocichla schistacea, Meyer, Zeitschr. f. ges. Orn. I, p. 211, Taf. VIII (Timor-laut) (1884) (Tipo esaminato). - Eorbes, Naturalist's Wand, p. 355 (1885). -- Sharpe, Gonld's B. New Guin. pt. XXIV, pl. 11 (1888). 
Supra sclistaceus, capite longitudinaliter nigro maculato; fronte, loris, gula pectoreque summo nigris; superciliis auricularibusque allis; subtus albus, pectore imo maculis nigris transversis notato; corporis lateribus griseis, tectricibus alarum nigris, minoribus schistaceo, mediis et majoribus albo marginatis; remigibus rectricibusque nigricantibus, griseo linbatis, rectricibus duabus extimis maculis apicnlibus albis ornatis; rostro nigro; pedibus flavescentibus.

Long. tot. $0^{\mathrm{m}}, 170$ : al. $0^{\mathrm{m}}, 093-0^{\mathrm{m}}, 100$; caud. $0^{\mathrm{m}}, 068-0^{\mathrm{mm}}, 073$; rostri $0^{\mathrm{m}}, 020$. $0^{\mathrm{m}}, 022$; tarsi $0^{\mathrm{m}}, 025-0^{\mathrm{m}}, 027$.

Hab. in Papuasia - Timor-laut (Riedel).

Questa specie somiglia molto al Turdutus wardi (Jerd.) dell'India, ma questo ha le parti superiori nere e le piume auricolari non bianche, ma nere.

Lo Sharpe fa notare cọme le differenze fra il $T$. machiti ed il $T$. schistaceus siano analoghe a quelle che si osservano fra la femmina ed il maschio del $T$. wardi, e tuttavia non sia possibile considerare il $T$. machiki ed il $T$. schistacens come femmina e maschio di una medesima specie, essendo il primo tanto più grande del secondo.

(636) Monticola solitarius (P. L. S. MüLL.).

Monticola solitaria, Seebh., Cat. B. V, p. 319 (1881).

Il Seebohm non cita il Turdus erythropterus, Gray, fra i sinonimi di questa specie.

\section{(637) Megalurus macrurus (SALVAD.)}

Megalurus macrurus, Sharpe, Cat. B. VII, p. 126 (1883), - Finsch, Vög. d. Sïdsee, p. 11 (Nivova Britannia e Nuova Guinea) (1884).

(638) Megalurus amboinensis (Salvad.).

Megalurus amboinensis, Sharpe, Cat. B. VII, pp. 127, 671 (1883 - Forbes, P. Z. S. 1883, p. 589 (Amhoina).

Lo Sharpe crede che gli esemplari di Amboina non differiscano specificamente dal M. galactodes (Temm.) dell'Australia.

(639) Poodytes albolimbatus, D'Alb. et Salvad.

Megalurus albolimbatus, Sharpe, Cat. B. Brit. Mus. VII, p. 129 (1883). — Id., Gould's B. New Guin. pt. XVI, pl. 2 (1884).

$(640,641,642)$ Cisticola exilis (V. et H.).

Malurus exilis, Vig. et Horsf., Trans. Linn. Soc. XV, p. 223 (1826 ex Lath. Mss.).

Gisticola ruficeps, Sharpe, Journ. Linn. Soc., Zool. XVI, p. 442 (Morocco) (1882) - Finsch, Vög. d. Südsee, p. 11 (Alova Britannia), p. $3 \hat{3}$ (Torres Str.) (1884)。 - Ramsay, Tab. List, p. 9 (1888).

Cisticola exilis, Sharpe, Cat. B. VII, pp. 269, 671 (1883).

Lo Sharpe riferisce a questa specie non solo la $C$. ruficeps, Gould, la C.rustica, Wall. e la Cisticola sp. delle Isole del Duca di York (Om. Pap. e Mol. II, p. 424), ma molte altre supposte specie, quali la $C$. lineocapilla, Gould, d'Australia, fondata sulle femmine e sui maschi in abito invernale, la $C$. isura, Gould, pure d'Australia, che sarebbe la femmina colla coda quadrata nell'alito estivo, la C. erythrocephala, Blyth di Coorg nell'India, la C. volitans, Swinh. della Formosa, la C. tytleri, Jerd. del- 
l'Assam, la $C$. semivefa, Cab. di Luzon, la C. delicatula, Blyth, di Giava, la C. melanocephala, Anders. del Yunan, lin C. ruficollis, Wald. di Celebes, e la C. grayi, Wald., e perciò l'area di questa specie si estenderebbe dall'Australia, per le isole della Papuasia e delle Molucche (della Sondla?) e delle Filippine fino nella Formosa, nella penisola di Malacca, nel Burmah, nell'Assam e nel Bengala orientale!

(643) Calomodyta orientalis (T. et S.).

Acrocephalus orientalis, Seebh., ('at. B. V, p. 97 (1881)

(644) Calamodyta australis (GovLD).

Acrocephalus australis, Seebh., Cat. B. V, p. 100 (1881).

Il Seebohm non menziona Bu'u, fra le localita abitate da questa specie, che vi fu trovata dal Wallace (F. Z. S. 1863, p. 25), ed annovera invece un esemplare di Lombock, che dice raccolto dal Wallace; ma mentre la prima ommissione è evidente, io sospetto che la seconda asserzione sia erronea.

(645) Locustella fasciolata (G. R. GR.).

Locustella fasciolata, Seebh., Cat. B. V. p. 108, pl. V (1881). - Pleske, Bull. Acad. Petersb. XXIX, p. 532 ('Ternate) (1884).

(646) Phylloscopus borealis (BLAs.).

Phylloscopus borealis, Seebl., Cat. B. V, p. 102 (Norty Waillace) (1881). - Neyer, Sitzb. u. Abh. Gesellsch. Isis, 1884, Abh. 1, p. 47 (Sangi Meyer, Ceran Riedel). - Pleske, Bull. Acad. Petersb. XXIX, p. 532 (Ternate) (1884).

(647) Budytes flavus (LiNN.).

Budytes viridis, Salvad., Voy. Chall., Birds, p. 63 (1881) -- Pleske, Bull. Acad. Petersb. XXIX, p. 532 (Ternate) (1884).

Motacilla flava, Sharpe, Cat. B. X, p. 315 (1885) (Ceram).

Budytes flavescens (Shaw). - Homeyer, Journ. f. Orn. 1878, p. 131.

Hab. Ceram (Wallace).

Lo Sharpe ( $($. c.) afferma che della Motacilla flava esiste una colonia orientale, che si estende dall'Alaska e dalla Siberia settentrionale alla Cina, e che emigra in inverno fino nelle Molucche; io seguo ora lo Sharpe, non avendo potuto esaminare esemplari delle Molucche in abito perfetto.

\section{(648) Calobates melanope (PALL.).}

Calobates melanope, Pleske, Bull. Acad. Pelersb. XXIX, p. 532 (Ternate). - Guillem., P. Z. S. 1885, p. 644 (Waigiou.)

Motacilla melanope, part., Sharpe, Cat. B. X, p. 467 (1885) (Norty Isl., Amboina Wallace).

(649) Corydalla gustavi (SwINH.).

Gorydalla gustavi, Pleske, Bull. Acad. Petersb. XXIX, p. 533 (Ternate) (1884).

Anthus gustavi, Sharpe, Cat. B. X, p. 613 (1885) (Timor Wullace). - fuillem., P. Z. S. 1885, p. 272 (Batchian),

\section{Sp. 1127 (649 bis) Corydalla richardi (VieiLı.).}

Anthus richardi, Vieill., N. D. XXVI, p. 491 (1818), - Blyth, Cat. K. Mus. As. Soc. Beng. p. 135, n. 755 (1849), - Ilorsf, ot Moore, Gat. B. Mus. E. I. Comp. I, p. 355, n. 563 (1854). - G. R. Gr. [264] 
Hand-List, I, p. 252, n. 3649 (1869). - Dress., B. of Eur. III, p. 325, pl. 138(1875). - Seehh., Ibis, 1878, p. 343. - Sharpe, Cat. B. Brit. Mus. X, p. 564 (Nysol) (1885).

Corydalla richardi, Vig., Zool, Journ. II, p. 397 1826). - Jerd., B. of Ind. II, p. 231 (1863). Swinh., P. Z. S. 1871, p. 366. - Blyth et Wald., B. Burm. p. 95 (1875). - Prjew., in Kowl. Orn.

Misc. II, p. 195 (1877) - - Javid et Oust. Ois. Chin. p. 309 (1877). - Legge, B. Ceyl. p. 621 (1879).

- Hume, Str. leath. 1879, p. 103. - Oates, B. Brit. Burm. 1, p. 166 (1883).

Corydalla sinensis, Bp., Consp. I, p. 247 (1850). - David et Oust., Ois. Chin. p. 311 (1877).

Anthus richardi var., G. R. Gr., P. Z. S. 1861, p. 634 (Mysol).

Corydalla chinensis, Swinh., P. Z. S. 1871, p. 366.

Rufescens, plumis corporis superioris in medio fuscis; superciliis, pectore et lateribus rufescentibus; gula, abdomine ct subcaudalibus albidis; macula suboculari et linea mystacali fuscis; gutture nigro maculato; remigibus fuscis, rufescente marginatis; rectricibus fuscis, mediis duabus rufescente marginatis, extimis duabus magna ex parte albis; maxilla fusca, mandibula pallida; pedibus rufescente-caneis.

Foem. Minor.

Long. tot. $0^{\mathrm{m}}, 195$; al. $0^{\mathrm{m}}, 100$; caud. $0^{\mathrm{m}}, 085$; rostri $0^{\mathrm{m}}, 014$; tarsi $0^{\mathrm{m}}, 031$; ung. dig. post. $0^{\mathrm{m}}, 021$.

Hab. in Asia centrali et orientali (Prjewalski, Dybowski); Sibiria (Seebohm); Sina (Swinhoe, David); India (Jerdon, Hume); Ceylon (Legge); Burma (Oates) in Papuasia - Mysol (Wallace); Europa et Africa septentrionali.

Questa specie si riconosce facilmente alle sue grandi dimensioni, al tarso molto lungo ed all'unghia del dito posteriore pure molto lunga e talora superante la lunghezza del dito.

Essa nidifica nell'Asia centrale ed orientale ed anche nella Siberia, donde in autunno emigra nell'India, nella Birmania e nella Cina; una sola volta è stata trovata nella sottoregione papuana, cioè in Mysol dal Wallace; verso occidente si estende fino in Europa; raramente è stata trovata nell'Africa settentrionale.

Questa specie è stata ommessa da me nella seconda parte della Ornitologia, essendomi sfuggita la citazione del Gray, relativa all'esemplare di Mysol; quella importante citazione è sfuggita anche allo Sharpe (1. c.).

\section{Mirafra javanica, Horsf.}

Il Meyer, Sitzb. u. Abh. Ges. Isis, 1884, Abh. I, p. 47 (Reichen., Bericht, Naturg. Vög. 1884, p. 213), menziona un esemplare della Mirafra javanica, Horsf., inviato al Museo di Dresda dal von Schierbrand come proveniente da Ceram; sebbene questa cosa non. sia al tutto impossibile, tuttavia sicconıe nessuno ha mai trovato alcuna specie della famiglia delle Alaudidae nelle MIolucche, e le indicazioni date dal von Schierbrand, rispetto alla provenienza degli esemplari da lui inviati, non sempre somo esatte, credo che convenga di attendere prove ulteriori prima di ammettere la M. javanica, Horsf. fra le specie delle Molucche.

\section{(650) Munia molucca (Linn.).}

Munia molucca, Meyer, Verb. z.-b. Ges. Wien, 1831, p. 768 (Timor). - Blas. u. Nehrk., Verh. Z.-b. Ges. Wien, 1882, p. 427 (Amboina). - Sclat. P. Z. S. 1883, pp. 51, 195, 200 (Larat Forbes, Pleske, Bull. Acad. Petersb. XXIX, p. 533 (Ternate) (1884). - Forbes, P. Z. S. 1884, p. 433. Neyer, Zeitschr. f. ges. Orn. I, p. 194 (1884). 
? Munia sp., Meyer, Zeitschr. f. ges. Orn, I, p. 292 (Aru) (1884).

Il Meyer fa notare che dalle Isole Aru, delle quali non si conosce alcuna specie di Mrnia, egli arrebbe riceruto un nido con tre uova di una specie indeterminata. Non è improbabile che si tratti della specie presente.

(654) Munia forbesi, Sclat.

Munia forbesi, Bartl., Mon. Ploc. etc. pt. V, pl. VIlI, f. 2 (1889).

(655) Munia melaena Sclat.

Munia melaena, Bartl., Mon. Ploc. etc. pt. V, pl. VIII, f. 1 (1889).

(10l6) Munia grandis, Sharpe.

Munia grandis, Sharpe, Gould's B. New Guin. pt. XIV, pl. 7 (1883).

Questa specie, notevole per la sua grandezza, ha tutta la testa e le parti inferiori, trunne i fianchi, nere, i fianchi, il dorso e le ali castagne, il groppone, il sopraccoda ed i margini delle timoniere di color giallo paglia, alquanto dorato.

\section{Sp. 1128 (656 bis) Donacicola hunsteini, Finsch.}

Donacicola hunsteini, Finsch, Ibis, 1886, p. 1, pl. I. - Finsch u. Meyer, Zeitschr, f. ges. Orn 1886, p. 26. - Sharpe, Gould's B. New Guin. pt. XXIV, pl. 9 (1888).

Nigerrima; pileo, cervice et lateribus colli griseo-cinereis, plumarum basi fuscis; loris et genis anticis nigris, plumarum apicibus griseis; uropygio, supracaudalibus et rectricibus mediis castaneis, flavo-aureo tinctis; rectricibus lateralibus fuscis; subalaribus pallide rufis; rostro et pedibus nigris; iride fusca.

Long. tot. $0^{\mathrm{m}}, 088$; al. $0^{\mathrm{m}}, 050$; caud. $0^{\mathrm{m}}, 029$; rostri $0^{\mathrm{n}}, 010$; tarsi $0^{\mathrm{m}}, 015$.

Hab. in Papuasia - Nova Hibernia (Finsch).

Questa specie è ben distinta pei margini grigi chiari delle piume del capo, le quali presentano quasi un disegno a squame.

Dice il Finsch che gli esemplari perfettamente adulti hanino le piume della gola con sottili margini grigi, i quali formano talora una sorte di semicollare interrotto.

Il giovane è bruno scuro, variegato di nero e di fulvo sulle parti inferiori; sulla cerrice ha talora piume marginate di grigio; il sopraccoda e la coda sono di color bruno uniforme.

Secondo il Finsch, questa specie vive in mezzo alle alte erbe.

(657) Donacicola spectabilis, Sclat.

Donacicola spectabilís, Finsch, Vög. d. Südsee, p. 14 (Nova Britannia) (1884).

Munia spectabilis, Barll., Mon. Ploc. etc. pt. V, pl. VIII, f. 3 (1889).

\section{(659) Erythrura trichroa (KittL.).}

Erythrura trichroa var., Ransay, Pr. Linn. Soc. N. S. W. vilI, p. 20 (1883). - Salvad., Ibis, 1884, p. 354.

Ery ythrura trichroa, Pleske, Bull. Acad. Petersb. XXIX, p. 533 (Ternate) (1884). - Guillem., P. Z. S. 1885, p. 573 (Ternate). - Grant, P. Z. S. 1888, p. 197.

Hab. in ins. Salomonis, Guadalcanar (Woodford).

[266] 


\section{Sp. 1129 (659 bis) Erythrura tricolor (VIEILL.)}

Azuvert, Vieill., Ois. Chant. pl. 20 (1805). - Less., Compl. de Buff. Il, p. 346 (1837).

Fringilla tricolor, Vieill., N. U. XII, p. 233 (1817) - Id., Enc. Méth. III, p. 974 (1823).

Amadina prasina, part., G. R. Gr., Gerr. B. III, p. 370 (1849).

Fringilla sylvestris, Temm., fide Bp., Consp. I, p. 457 (1850).

Erythrura tricolor, Bp., Consp. 1. p. $457(1850)$ - - Hartl., P. Z. S. 1858, p. 462. - Wall., P. Z

S. 1863 , p. 486, n. 135. - G. R. Gr., Hand-List, II, p. 58, n. 6807 (1870). - Sclat., P. Z. S. 1883,

pp. 51, 57, 290 (Loetoe). - Meyer, Sitzb. 1. Abh. Gesellsch. Isis, 1884, Abh. 1, p. 48 (Babbar).-

Id., Zeitschr. f. ges. Orn. I, p. 194 (1884)

Diachmura tricolor, Rchb., Singv. p. 48, Taf. XVII, fig. 156 (rect. 157) (1863) (ex Vieillot).

Erythrura trichroa, Forbes (nec. Kittl.), P. Z. S. 1884, p. 433.

Laete viridis, fronte, genis et gastruco toto lacte cyaneis; supracaudalibus et cauda supra rubris.

Long. al. $0^{\mathrm{m}}, 053$; rostri $0^{\mathrm{m}}, 010$.

Hab. in Timor (Vieillot, Wallace); Babbar (Riedel); Loetoe (Forbes).

Il Vieillot ha figurato e descritto questa specie colla coda verde, ma tanto nel Nouveau Dictionnaire, quanto nella Encyclopédie, egli non menziona il colore della coda; il Bonaparte e l'Hartlaub descrivono la coda di color rosso, e quindi il Reichenbach, il quale ha copiato il Vieillot, ha creduto che essi fossero in errore, laddove l'errore fu commesso dal Vieillot, ed è tale che io non so spiegarmelo. Disgraziatamente il Pucheran non menziona questa specie fra i tipi del Museo di Parigi.

\section{(660) Chlorura hyperythra, RCHB.}

Io annoverai questa specie in modo dubbio fra quelle della Nuova Guinea, e sulla fede del Reichenbach, il quale asseriva di averne ricevuto un esemplare dal von Schierbrand, insieme con molti altri uccelli della Nuova Guinea. Ora questa specie è da escludere dal novero degli uccelli di questa regione, avendo il Vorderman dimostrato che essa è propria di Giava (Natuurl. Tijdschr. Neilerl. Ind. XLII, p. 248).

(661) Neochmia phaeton (H. et J.).

Estrilda (Neochmia) phaeton, Ramsay, Tab. List, p. 10 (partim?) (1888).

\section{(663) Calornis metallica (Tems.).}

Calornis metallica, Salvad., Voy. Chall., Birds, pp.70, 81 (1881). - Ramsay, Pr. Linn. Soc. N. S. W. VII, p. 25 (1882) (Isole Salomone) - Tristr., Ihis, 1882. pp. 137, 143. - Blas. 1. Nerk., Verh. z.-b. Gesellsch. Wien, 1882, p. 427 (Amboina). - Finsch, Vög. d. Südsee, p. 13 (Nova Britannia), 36 (1884). - Neyer, Sitzb. u. Abh. Gesellsch. Isis, 1884, A bh. I, p. 48 (Jobi). - Id., Zeitschr. f. ges. Orn. I, p. 292 (1884). - Pleske, Bull. Acad, Petersb. XXIX p. 533 (Ternate) (1884). - Guillem., P. Z. S. 1885 , p. 573 (Ternate), 644 (Mysol, Salwatti, Aru). - Grant, P. Z. S. 1887, p. 331 (Alu, Fauro). - Ramsay, Tab. List, p. 12 (1888).

Il Guillemard ha raccolto diversi esemplari in Mysol, che converrebbe esaminare per risolvere la questione se la C. gularis, Gr. di Mysol sia o no specificamente diversa.

Sp. 1130 (663 tar) Calornis circumscripta, MEYER.

Calornis metallica, Sclat. (uec Temm.), P. Z. S. 1883, n. 51 (Maroe), 195 (Larat, Kirimoen), 200. Calornis circumscripta, Meyer, Sitzb. 1, Abh. Gesellsch. Isis, 1834. Abh. 1, p. 49 (Timorlaut).Salvad., Ibis, 1884, p. 355. - Id., P. Z. S. 1884, p. 579.

Calornis gularis, Forbes (nec Gray), P. Z. S. 1884, pp.429, 430.433. - Id., Nat. Wand. E. Arch. p. 365 (1885). - Sharpe (nec Gray), Gould's B. Yew Guin. pt. XX, pl. 12 (1885). 
Nitidissime viridis, alis et cauda obscurioribus; capite, interscapulio, fasciu utrinque gulari cum capite post aures conjuncta et fascia antepectorali cum interscapulio conjuncta pulchre purpureis; fascia malari et torque cervicali nitidissime viridibus; rostro pedibusque nigris; iride rubra.

Foem. (an pot. juv.?). Supra mari similis, sed minus nitens; subtus alba, plumis in medio nitide viridibus.

Long. tot. $0^{\mathrm{m}}, 210-0^{\mathrm{m}}, 240$; al. $0^{\mathrm{m}}, 098-0^{\mathrm{m}}, 110$; caud. $0^{\mathrm{m}}, 095-0^{\mathrm{m}}, 120$; rostri $0^{\mathrm{m}}, 017-0,{ }^{\mathrm{m}} 020$; tarsi $0^{\mathrm{m}}, 020-0^{\mathrm{nd}}, 022$.

Hab. in Papuasia - Timor-laut (Forbes, Riedel).

Questa specie è affine alla C. metallica, dalla quale differisce principalmente per le due linee di color porporino che scorrono ai lati della gola sotto le branche della mandibola, incontrandosi sul mento per modo da formare un $\Lambda$; inoltre essa ha il collare verde cervicale più stretto, e la grande area porporina sulla regione interscapolare senza la macchia triangolare verde nel mezzo, o appena percettibile in alcuni esemplari, laddore quella macchia è generalmente molto cospicua nella $C$. metallicu.

Gli esemplari di Timor-laut furono da prima riferiti alla C. metallica dallo Sclater, poscia essi furono distinti specificamente dal Meyer e finalmente il Forbes e lo Sharpe hanno creduto di doverli riferire alla C. gularis, G. R. Gr. di Mysol, nota per un solo esemplare conservato nel Museo Britannico, ma io ho fatto gia notare di non poter convenire con quella identificazione, e di considerare gli esemplari di Timorlaut specificamente distinti, come ha fatto il Meyer. Io ho già espresso l'opinione che il tipo della $C$. gularis, da me attentamente esaminato, non sia altro che una varieta individuale della $C$. metallica, avente più di porporino sulla gola, come altri esemplari di Halmahera e del Capo York, da me esaminati; ma potrebbe essere clıe l'esame di un maggior numero di esemplari di Mysol dimostrasse che essi differiscono costantemente dagli esemplari tipici della $C$. meta7lica, ed infatti il Guillemard fa notare che quelli da lui raccolti in Mysol erano più brillanti di quelli delle isole Aru; non è quindi improbabile che la $C$. gularis di Mysol si debba ammettere come specie distinta.

Rispetto alla. C. circumscripta io credo che essa sia una forma insulare-della largamente diffusa $C$. metallica, come la $C$. purpureiceps delle Isole dell'Ammiragliato e come la $C$. inornata di Mysori, e non riferibile alla C. gularis, e chiunque rifletta a questo fatto che le forme insulari sono limitate ad una isola od a quelle di un medesimo gruppo, converra meco nel non poter credere possibile che la stessa specie si trovi in Mysol ed in Timor-laut, tanto lontane l'una dall'altra, mentre la vera C. motallica vive in tante isole poste fra esse.

(664) Calornis purpureiceps, Salvad.

Calornis purpureiceps, part., Sclat., Voy. Chall., Birds, p. 29 (1881). - Forbes ,P. Z. S. 1884, p. 430.

(667) Calornis obscura (Forsten).

Calornis obscura, part. (3), Nehrk., Jour!!. f. Orn. 1885, p. 33 (Waigeu). - Guillem., P. Z. S. 1885, p. 573 (Batchian).

È possibile che alcuni degli esemplari menzionati dal Nehrkorn non appartengano veramente a questa specie. 
(668) Calornis cantoroides, G. R. GR.

Calornis purpureiceps, part., Sclat., Voy. Chall., Birds, p. 29 (specim. 467) (1881).

Calornis cantoroides, Ramsay, Pr. I.inn. Soc. N. S. W. VIl, p. 26 (1882) (Isole Salomone). Tristr., Ibis, 1882, pp. 137, 143. - Finsch, Vög. d. Sïdsee, p. 14 (Nova Britannia) (1884). - Meyer, Sitzb. u. Abh. Gesellsch. Isis, 1884. Abh. I, p. 48 (Aru Ried - - Guillem., P. Z. S. 1885, p. 644 (Mysol, Salwatti). - Meyer, Zeitschr. f. ges. Orn. 1886, p. 35 (Jamma). - (irant, P. Z. S. 1887, p. 331 (Alu).

Calornis obscura, part., Nehrk. (nec 6. R. Gr.), Journ. f. Orn. 1885, p. 33. - Salvad., Ibis, 1886 , p. 152.

Uno dei tre esemplari menzionati dal Nehrkorn è stato da me esaminato ed esso appartiene alla $C$. cantoroides.

\section{Sp. 1131 (668 bis) Calornis crassa, Sclat.}

Galornis crassa, Sclat., P. Z. S. 1883, pp. 51, 56, 195, pl. XIV. - Sharpe, Gould's B. Kew Guin. pt XV, pl. 10 (1883). - Forbes, P. Z. S. 188', p. 433. - Neyer, Zeitschr. f. ges. Orn. I, p. 194 (1884). - Büttik, Not. Leyd. Mus. VIII, p. 65 (1886).

Obscure cineraceo-viridis nitore chalybeo; subtus, praecipue in ventre, pauto magis cineracea; alis caudaque nigris exterius dorsi colore lavatis; remigum marginibus interioribus fuliginosis; rostro et pedibus nigris; cauda fere aequali aut pautum rotundata; iride brunnea (Sclater).

Foem. Supra cineracea, vix viridi nitens, striis scaporum nigris variegata; alis caudaque fusco-nigris; subtus alba, fusco-cinereo flammulata.

Long. tot. $0^{\mathrm{m}}, 188$; al. $0^{\mathrm{m}}, 101$; caud. $0^{\mathrm{m}}, 073$; rostri $0^{\mathrm{m}}, 020$; tarsi $0^{\mathrm{m}}, 024$.

Hab. in Papuasia - Ins. Tenimberensibus, Larat (Forbes, Riedel).

Questa specie, dalla coda mediocre e rotondata, si distingue pel colorito verdecineraceo splendente.

Potrebbe essere che gli esemplari descritti come femmine fossero i giovani; io ho visto uno di tali esemplari raccolti dal Riedel ed inviato al Museo di Torino dal Dr. Meyer.

(1017) Calornis feadensis, Ramsay.

Galornis feadensis, Sclat, P. Z. S. 1883, p. 3ł7. - Sharpe, Gould's B. New Guin pt. XXI, pl. 11 (1886).

Questa specie ha la coda breve e non graduata.

(670) Lamprocorax grandis, SALVAD.

Sturnoides fulvipennis, Ramsay, Pr. Linn. N. S. W. VII, p. 26 (1882) (Isole Salomone).

Calornis fulvipennis, Tristr., lbis, 1882, pp. 137, 143.

Lamprotornis fulvipennis, Ramsay, l. c. p. 668 (1882).

Lamprocorax grandis, Grant, P. Z. S. 1887, p. 331; 1888, p. 198.

\section{(1018) Lamprocorax minor (RAMSAY).}

Galornis fulvipennis, Tristr. (nec Jacq. et Pucher.), 1bis, 1882, p. 137.

Sturnoides minor, Ramsay, Pr. Linn. Soc. N. S. W. VII, p. 26 (1882, (St. C'ristoval).

Sturnoides (Lamprotornis) minor, Ramsay, op. cit. p. 668 (1882).

Lamprocorax grandis, Sharpe (nec Salvad.), Gould's B. New Giuin. pt. XXIII, pl. 6 (1887).

Lamprocorax minor, Sharpe, ibid. text. pl. 6 (1887).

19 T. Salvadori. 
(672) Melanopyrrhus anais (Less:).

Melanopyrrhus anais, Guillem., P. Z. S. 1885, p. 644 (Salwatti). - Sharpe, Gould's B. New Guin. pt. XXII, pl. 7 (1886).

(673) Melanopyrrhus orientalis (SCHLEG.).

Melanopyrrhus orientalis, Guillem., P. Z. S. 1885, p. 044 (Arfak). - Sharpe, Gould's B. New Guin. pt. XXII, pl. 8 (1886). - Meyer, Zeitschr. f. ges. Orn. 1886, p. 35 (Kafu).

亡̀ poco probabile che l'esemplare avuto dal Guillemard provenisse dai Monti Arfak, essendo questa specie propria di una regione più orientale.

(674) Mino dumonti, Less.

Mino dumonti, Salvad. Voy. Chall., Birds, p. 81 (1881). - Sharpe, Journ. Linn. Soc., Zool. XVI, p. 442 (East Cape) (1882). - Fiusch, Vōg. d. Südsee, p. 27 (1884). - Nehrk., Journ. f. Orn. 1885, p. 33 (Waigeu). - Guillem., P. Z. S. 1885, p. 645 (Waigiou, Batanta, Dorei, Aru). - Meyer, Zeitschr. f. ges. Orn. 1886, p. 35 (Kafu). - Tristr., Ibis, 1884, p. 555.

Prima del Guillemard, questa specie non era stata indicata di Batanta.

Gli esemplari di Kafu, secondo il Meyer, sono più azzurri, specialmente sulla gola e sul petto, e sono simili per quel rispetto a quelli di Jobi e delle isole Aru, mentre quelli di Passim nella Nuora Guinea sarebbero verdi.

\section{(675) Mino kreffti (Sclat.).}

Gracula krefti, Ramsay, Pr. Linn. Soc. N. S. W. VII, p. 25 (1882)(Isole Salomone). - Tristr, Ibis, 1882, p. 142 .

Mino kreffti, Finsch, Vög. d. Südsee, p. 14 (Nova Britannia). - Grant, P. Z. S. 1887, p. 332 (Alu. Fauro); 1888, p. 198 (Guadalcanar).

(676) Mimeta flavocinctus, King.

Mimeta flavocincta, Meyer, Zeitschr. f. ges. Orn. I, p. 292, T'af. XVII, f. 1 (novo) (1884).

Il Meyer descrive anche il nido.

(677) Mimeta striatus (Q. et G.).

Oriolus striatus, Finsch, Vög. d. Südsee, p. 26 (Port Moresby) (1884).

Mimeta striata, Nehrk., Journ. f. Orn. 1885, p. 34 (Waigeu).

Mimeta striatus, Guillem., P. Z. S. 1885, p. 645 (Batanta).

\section{Sp. 1132 (679 bis) Mimeta decipiens, Sclat.}

Mimeta decipiens, Sclat., P. Z. S. 1883, pp. 195, 199. - Meyer, Zeitschr. f. ges. Orn. I, p. 194 (1884). Oriolus decipiens, Sharpe, Gould's B. New Guin. pt. XVI, pl. 12 (1884). - Forbes, P. Z. S. 1884, p. 432. - Büttik., Not. Leyd. Miss. VIII, p. 65 (1886).

Fusco-griseus, fere unicolor, pileo nigricanti striolato, subtus paullo ditutior; gula et cervice pallide cinercis, lateribus colli nigro guttulatis; pectoris summi plumis quibusdam nigricanti striolatis; regione auriculari nigricante; remigibus intus basin versus rufesconte tinctis; rostro fusco-brunneo; pedibus fuscis (cinereoplumbeis ?); iride fusco-brunnea.

Long. tot. $0^{\mathrm{m}}, 300$; al. $0^{\mathrm{m}}, 154$; caud. $0^{\mathrm{m}}, 127$; rostri $0^{\mathrm{m}}, 036$; tarsi $0^{\mathrm{m}}, 031$.

Hab. in Papuasia - Ins. Tenimberensibus (Forbes, Riedel). 
Secondo lo Sclater e lo Sharpe questa specie sarebbe affine al $\boldsymbol{M}$. bourocnsis, ma ne differisce per la gola bianchiccia con macchie nere e per le piume superiori del petto striate di nero.

Io ho esaminato tre esemplari di questa specie, raccolti dal Riedel e nessuno ha la testa, il collo ed il petto cosi distintamente macchiati come nella descrizione e nella figura date dallo Sclater e dallo Sharpe. Anche il Buttikofer fa notare che un esemplare maschio del Museo di Leida differisce dalla descrizione dello Sclater per i medesimi rispetti. Aggiungo che un esemplare del IIuseo di Torino ha la testa di un grigio bruno, nettamente distinto dal grigio delle piume della cervice, che sono alquanto ricurve come nel Plilemon plumigenis; inoltre esso non presenta traccia di strie scure sulle piume del petto.

(683) Corvus orru, S. MüLL.

Corvus orru, Finsch, Vög. đ. Südsee, p. 14 (Tova Britannia) (1884). - Pleske, Bull. Acad. Petersb. XXIX, p. 534 (Ternate) (1884). - Sehrk., Journ. f. Orn. 1885, p. 34 (Waigeu). - Guillem., P. Z. S. 1885, p. 645 (Waigiou, Neosmapi Isl. (Dorei Bay), Mysol, Salwatti).

\section{Sp. 1133 ( $683^{\text {bis }}$ ) Corvus latirostris, MeYer.}

Corvus validissimus, Sclat. (nec Schleg.), P. Z. S. 1883, pp. 51, 195, 200. - Salvad., Ibis, 1884, p. 355, - Forbes, P. Z. S. 1884, p. 433.

Corvus latirostris, Meyer, Zeitschr. f. ges. Orn. I, pp. 194, 199 (Timorlant) (1884) - Salrad., P. Z. S. 1884, p. 580.

Corvus macrorhynchus, Büttiks, Not. Leyd. Mus. VIII, p. 65 (1886).

Corvus C. orru similis, sed rostri basi latiore.

Supra nigro-coerulescens, subtus, alis, tectricibus alarum, primariis caudaque plus minusve virescenti tinctis; plumarum parte basali alba; plumis gutturis elongatis, lanceolatis; rostro pedibusque nigris.

Long. tot. $0^{\mathrm{m}}, 440$; al. $0^{\mathrm{m}}, 300$; caud. $0^{\mathrm{m}}, 180$; rostri culm. $0^{\mathrm{m}}, 060$; tarsi $0^{m}, 057$.

\section{Hab. in Papuasia - Timor-laut (Forbes, Riedel).}

Lo Sclater riferi questa specie al Corvus validissimus, Schleg., ma io feci notare la poca probabilità che la determinazione fosse esatta ed il Meyer la distinse poscia col nome di $C$. latirostris; secondo il Büttikofer, questa specie non sarebbe diversa dal C. macrorhynchus, Wagl., del quale il Museo di Leida possiede esemplari di Borneo, di Giava, di Sumbava, di Timor e di Wetter, e che, secondo lo Sharpe (Cat. B. III, p. 38) si trova anche in Malacca, in Sumatra, in Flores ed in Balì. 亡̀ molto probabile che la identificazione del Büttikofer sia esatta, ma per mancanza di suffcienti materiali di confronto, avendo esaminato soltanto il tipo del $C$. latirostris, e non avendo alcun esemplare riferibile al $C$. macrorhynchus, non sono in grado di decidere la questione.

Ciò che distingue il C. latirostris dal C. orru (MIüll.), cui pure quello è molto affine, è la larghezza notevolmente maggiore del becco, che è più largo (mm, 26) che non alto (mm. 22); inoltre ne differisce anche pei riflessi verdi della testa, del collo, delle parti inferiori, delle ali e della coda. 
(684) Corvus salvadorii, FinsCH.

Corvus sp., Salvad., Orn. Pap. e Mol. II, p. 486 (1881).

Gorvus salvadorii, Finsch, Vög. d. Südsee, p. 28 (1884).

Il Finsch ha voluto distinguere col mio nome la specie, che io descrissi, ma che per insufficienza di materiali lasciai inuominata (l. c.).

(685) Corvus violaceus, Forsten.

Corvus violaceus, Blas., P. Z. S. 1882, p. 708 (Ceram).

(686) Corvus validissimus, ScHLEG.

Corvus validissimus, Guillem., P. Z. S. 1885, p. 573 (Batchian).

(687) Macrocorax fuscicapillus (G. R. GR.).

Macrocorax fuscicapillus, Meyer, Zeitschr, fo ges, Orn. I, p. 292 (1884).

Corvus validissimus, Nehrk. (nec Schleg.), Journ. f. Orn. 1885, p. 34 (Waigeu). - Salvad., Ibis, 1886, p. 152.

Sp. 1134 (687 bis) Macrocorax woodfordi, Grant.

Macrocorax woodfordi, Grant, P. Z. S. 1887, p. 332, pl. XXVII (Solomon Islands); 1888, p. 198. - Woodford, P. Z. S. 1888, p. 249.

Nigerrimus, capite, collo et gastraco retiquo viridi nitentibus; corpore reliquo purpureo-caeruleo nitente; rostro albido (interdum carneo), apice nigro; pedibus nigris; iride obscure grisea.

Long. tot. $0^{\mathrm{m}}, 395$; al. $0^{\mathrm{m}}, 261$; caud. $0^{\mathrm{m}}, 127$; rostri culm. $0^{\mathrm{m}}, 058$; tarsi $0^{\mathrm{m}}, 050$.

Hab. in ins. Salomonis, Guadalcanar (Woodford).

Il Woodford ha inviato una numerosa serie d'individui, tutti simili fra loro; la femmina non differisce dal maschio.

Non mi sembra che questa specie appartenga veramente al genere Macrocorax, non arendo il becco col culmine cosi arcuato; inoltre il colore chiaro del becco ne lo allontana e lo arvicina al Gymnocorax senex; forse esso è intermedio a questa specie ed al Mucrocorax fuscicapitlus.

\section{(688) Gymnocorax senex (Less.).}

Gymnocorax senex, Guillem., P. Z. S. 1885, p. 645 (Jobi).

(689) Lycocorax pyrrhopterus (Forsten).

Lycocorax pyrrhopterus, Eudes-Deslongch., Ann. Mus, d'Hist. Nat. Caen, I, p. 46 (1880). D’Hamonv., Bull. Soc. Zool. de Fr. 1886, p. 510.

Manucodia pyrrhopterus, Ilusschenbr., Dagboek, pp. 197, 230 (1883 - - Rosenb., Nitt. Om. Ver. Wien, 1885, p. 40.

\section{(690) Lycocorax obiensis, BERnst.}

Lycocorax obiensis, Eudes-Deslongch., Ann. Mus. d'Hist. Nat. Caen, 1, p. 47 (1880) - Guillem., P. Z. S. 1885 , p. 573 (0bi). - D'Ifamonv., Bull. Soc. Zool. de France, I, p. 510 (1886). - Sharpe, Gould's B. New Guin. pt: XXIV, pl. 7 (1888).

Manucodia obiensis, Nusschenbr, Dagboek, pp. 193, 230 (1883). - Rosenb, Mitth. Orn. Ver. Wien 1885, p. 40 . 
Tanto il Guillemard, quanto lo Sharpe fanno notare che anche in questa specie, almeno nei maschi, si osserva che la base del vessillo interno delle remiganti è bianca; questo carattere mancava in una femmina.

(691) Lycocorax morotensis, Bernst.

Lycocorax morotensis, Eudes-Deslongch. Anת. Nus. d'llist. Nat. Caen, I, p. 47 (1880). - D'Hamonv., Bull. Soc. Zool, de France, 1886, p. 510.

Manucodia morotensis, Nusschenbr., Dagboek, pp. 197, 230 (1883).

Manucodia morotensa (sic), llosenb, Mitth. Orn. Ver. Wien, 1885, p. 40.

(692) Manucodia comriei, SCLAT.

Manucodia Comrii, Eudes-Deslongch., Ann. Mus, d'llist. Nat. Caen, I, p. 44 (1880). - Musschenbr, Dagboek, pp. 196, 230 (1883). - Rosenh., Mitth. Orn. Ver. Wien, 1885, p. 40. - D'Hamonv., Bult. Soc. Zool. de Fr. 1886, p. 510 - Tristr., Ibis, 1889, p. 554.

(693) Manucodia chalybeata (Penn.).

Manucodia chalybata (sic), Eudes-Deslongch., Ann. Mus. d'Ilist. Aat. Caen, I, p. 43 (1880).

Manucodia viridis, Musschenhr., Danglioek, pp. 195, 228 (1883). - Rosenb., Mitth. Orn. Ver. Wien, 1885, p. 40.

Manucodia chalybeata, (iuillem., P. Z. S. 1885, p. 640 (Nysol). - Meyer, Zeitschr, f. ges, Orn. 1885, p. 37\%。 - Salvad., Ibis, 1886, p. 155. - Finsch et Nleyer, Ibis, 1886, pp. 241, 249

Manucodia chalybea, D'Hamonv., Bul!. Suc. Zool. de Fr. 1886, p. 510.

La opinione emessa dal Guillemard che la $M I$. chalybeatr e la II. atra siano l'una l'adulto e l'altra il giovane di una medesima specie non è sostenibile nè per l'esame dei caratteri che esse presentano, nè per la loro diversa distribuzione geografica, mancando la $\boldsymbol{M}$. checilybeata nelle isole Aru.

\section{Sp. 1135 (693 bis) Manucodia rubiensis, Merer.}

Munucodia rubiensis, Meyer, Zeitschro foges, Orn. 1885, p. 374; 1886, p. 36 (Kafu) - Id., Ibis, 1886, p. 242. - D'llamonv., Bull. Soc. Zool. de Fr. 1886, p. 510.

BInncodia M. clialybeatae (Penn.) simillima, sed minor.

Long. al. $0^{\mathrm{m}}, 155-0^{\mathrm{m}}, 160$; caud. $0^{\mathrm{m}}, 126-0^{\mathrm{m}}, 128$; rostri $0^{\mathrm{m}}, 032-0^{\mathrm{m}}, 033$.

Mab. in Papuasia - Nova Guinea, Rubi (MLyer); Kafu (Laglaize).

Secondo il Meyer, le Manucodie delle vicinanze di Rubi, luogo posto al fondo della Baja del Geelwink, si distingnerebbero per la loro piccolezza da quelle degli altri luoghi.

Inoltre in esse la parte inferiore del collo non appare verde, ma azzurra, e l'increspatura delle piume è pochissimo sviluppata. Nelle dimensioni, ciò che più colpisce sarebbe la piccolezza del becon, che è poco più grande di quelío della Phonygana keraulremi.

Non ho potuto esaminare alcun esemplare di questa specie.

(691) Manucodia jobiensis, SALvad.

Manucodia jobiensis, Eudes-Deslongch., Ann. Mus. d'Hist. Xat. Caen. I, p. 45 (1880). - Musschenbr., Dagboek, pp. 196, 229 (1883). - Rosenh., Mitth. Orn. Ver. Wien, 1885, p. 40. - Guillem.. P. Z. S. 1885, p.646 (Johi).-Meyer, Zeitschr. f. ges. Orn. 1885̄ p. 374. - Id., Ibis, 1886, p. 242. - D'Hamonv., Bull. Soc. Zool. de Fr. 1886, p. 510.

Le dimensioni attribuite dal Guillemard ad un esemplare di questa specie sono minori di quelle degli esemplari misurati da me e dal Neyer. 
(695) Manucodia atra (Less.).

Manucodia atra, Eudes-Deslongch., Ann. Mus, d'Hist. Nat. Caen, I, p. 45 (1880). - Salvad., Voy. Chall., Birds, p. 82 (1881). - Nusschenbr., Dagboek, pp. 196, 229 (1883). - ?Ramsay, Pr. Linn. Soc. N. S. W. VIII, p. 25 (ova) (1883). - Finsch, Vōg. d. Südsee, p. 28 (Port Moresby) (1884). Meyer, Zeitschr. f. ges. Orn. 1, p. 293 (1884). - Nehrk., Journ. f. Orn. 1885, p. 34 (Waigeu). Rosenb., Mitth. Orn. Ver. Wien, 1885, p. 40. - Guillem., P. Z. S. 1885, p. 646 Mysol, Waigiou, Dorei). - Meyer, Zeitschr. f. ges. Orn. 1885, p. 374. - Id., Ibis, 1886, p. 342. - D'Hamonv。, Bull. Soc. Zool. de Fr. 1886, p. 510.

\section{GeN. PHONYGAMA, Less.}

Forse è miglior partito quello di separare il genere Phonygama dal genere Manucodia.

\section{(696) Phonygama keraudreni (LESS. et GARN.).}

Phonygama keraudreni, Eudes-Deslongch., Ann. Mus. d'Hist. Nat. Caen, l, p. 41 (1880). D'Hamonv., Bull. Soc. Zool. de Fr. 1886, p. 510.

Manucodia keraudreni, Musschenbr., Daghoek, pp. 195, 228 (1883). - Rosenb., Mitth. Orn. Ver. Wien, 1885, p. 40.

\section{Sp. 1136 (696 bis) Phonygama purpureo-violacea, MEYER.}

? Phonygama jamesi, Sharpe, Cat. B. III, p. 181 (1877). - Id., Journ. Pr. Linn. Soc. XIII, p. 500 (1877). - Elliot, Ibis, 1878, p. 56. - Eudes-Deslongch., Ann. Mus. d’Hist. Nat. Caen, I, p. 42 (1880). - D'Hamonv., Bull. Soc. Zool. de Fr. 1886, p. 510.

? Manucodia Jamesii, Musschenbr., Dagboek, pp. 195, 229 (1883). — Rosenb., Mitth. Orn. Ver. Wien, 188j, p. 40.

Phonygama purpureo-violacea, Meyer, Zeitschr. f. ges. Orn. II, p. 375, Taf. XV (1885). - Id., Ibis, 1886, p. 242. - D'Hamonv., Bull. Soc. Zool. de Fr. 1886, 1. 510. - Sharpe, Gould's B. New Guin. pt. XXIII, pl. 10 (1887).

Phonygamae Kerandreni (Less. et Garn.) similis, sed dorso, alis caudaque purpureo-violaceis concoloribus et occipitis collique plumis valde elongatis distinguenda.

Long. tot. $0^{\mathrm{m}}, 300$; al. $0^{\mathrm{m}}, 167$; caud. $0^{\mathrm{m}}, 130$; rostri $0^{\mathrm{m}}, 030-0^{\mathrm{m}}, 031$; tarsi $0^{\mathrm{m}}, 034$.

Hab. in Papuasia - Nova Guinea meridionali-orientali, in montibus Astrolabii (Hunstein).

Tutte le parti superiori, tranne il collo e la testa, di un bel violetto-porporino, parti inferiori verdi con riflessi azzurri, specialmente sui fianchi; faccia inferiore delle ali e della coda nero-grigiastro; piume laterali della testa più lunghe di quelle dèlla $P h$. keraudreni, le piume del collo più larghe e notevolmente più lunghe e di color verde azzurrognolo, come tutta la testa, e cogli apici azzurri soltanto sulla cervice; la mandibola superiore è solcata longitudinalmente, della quale cosa vi è soltanto traccia nella $P \%$. Keraudreni; la base del becco è alquanto più stretta.

Questa specie differisce dalla $P h$. keraudreni per la uniformità della colorazione delle ali e del dorso, ed anche per lo splendore e pei riflessi del colorito, oltre che per le piume della testa e del collo molto più lunghe.

Dalla $P h$. Tunsteini, Sharpe, essa differisce per le dimensioni minori, simili a quelle della $P$ h. keraudreni, e per l'azzurro che manca affatto nella $P h$. hunsteini, la quale ha la testa di color verde-oliva, mentre è verde-azzurrognola nella $P h$. purpureo-violacea. 
Lo Sharpe ammette la distinzione fra la $P h$. purpureo-violaceu e la $P h$. keraudreni, ma dubita che la prima sia da riferire alla sua $P h$. jamesi; egli fa notare che le serie d'individui da lui esaminati condurrebbero a quella conclusione. La principale differenza fra esse consisterebbe in ciò che la $P h$. purpureo-violacea sarebbe più porporina superiormente e piu azzurrognola inferiormente, laddove il tipo della $P h$.jamesi è di colore verde-metallico superiormente ed azzurro-acciaio inferiormente; ma fra quegli estremi si trovano tutti i passaggi nella serie di esemplari esaminati; aggiunge anche lo Sharpe che il tipo della $P h$. jamesi è in muta e che mentre ha le vechie piume delle ali di color porporino poco splendente, le recenti sono di un bell'azzurro-porporino.

Finalmente io non so nascondere la mia impressione che la $P h$. purpurco-violacea non sia veramente diversa dalla $P h$. keraudreni, giacchè gli esemplari della Baja Hall da me esaminati, erano simili in tutto ad altri della Nuova Guinea meridionale-settentrionale. Si noti che la $P$. jamesi, alla quale ora lo Sharpe crede che si debba riferire la $P h$. purpureo-violacea, fu già da lui riconosciuta come identica colla Ph. keraudreni (Journ. Limn. Soc., Zoology, XVI, pp. 442, 443).

(1019) Phonygama hunsteini, Sharpe

Phonygama Hunsteini, D'Hamonv., Bull. Soc. Zool. de Fr. 1886, p. 510.

Sp. 1137 (1019 bis) Phonygama thomsoni (Tristr.).

Manucodia thomsoni, Tristr., lbis, 1889, p. 554.

Manucodia corpore prorsus toto purpureo-nitente; plumis dorsi, pectoris et abdominis longis et filamentosis; capite, mento et jugulo chalybaeo-viridibus; capite villoso, duabus cristis decorato; colli plumis mucronatis; rostro et pedibus nigris (Tristram).

Long. tot. 12.3 poll. Angl. $\left(=0^{\mathrm{m}}, 261\right)$; alae $7\left(=0^{\mathrm{m}}, 177\right)$; caud. $6\left(=0^{\mathrm{m}}, 152\right)$; tarsi $1.6\left(=0^{\mathrm{m}}, 040\right)$; rostri a rictu $1.45\left(=0^{\mathrm{m}}, 036\right)$.

Hab. in Ins. D'Entrecasteaux (Thomson).

Dice il Tristram che questa specie si può distingnere facilmente per la sua testa di color verde-oleoso splendente che contrasta notevolmente col violetto cupo del resto del corpo. Le cuopritrici delle ali sono di un violetto splendente, meno bello sul corpo per causa della densa massa di piume filamentose che lo ricoprono superiormente ed inferiormente. La trachea presenta strqordinarie circonvoluzioni sottocutanee.

Il Tristram ha ricevuto un solo esemplare di questa singolarissima specie.

(697) Parotia sexpennis (BODD.).

Parotia sefilata, Eudes-Deslongch., Ann. Nus. d'Hist. Nat. Caen, I, p. 35 (1880).

P aradisea sexpennis, Musschenbr., Dagboek, pp. 192, 225 (1883). -- Rosenb., Mitth. Orn. Ver. Wien. 1885 , p. 40.

Parotia sexpennis, Guillem., P. Z. S. 1885, p. 647 (Arfils). - D'Hamonv., Bull. Soc. Zool. de Fr. 1886, p. 510 .

\section{Sp. 1138 (667 bis) Parotia lawesii, RAMSAY.}

Parotia lawesii, Ramsay, Pr. Iinn. Soc. N. S. W. X, p. 243 (1885). - Id., Nature, 1885 (July), p. 288. - Finsch u. Meyer, Zeitschr. f. ges. Orn. II, p. 375, pl. 16 (1885). - Id., Ibis, 1886, p. 243. - D'Hamonville, Bull. Soc, Zool. de Fr. 1886, pp. 507, 510. - Sharpe, Nature, vol. 34, p. 340 (1886). - Id., Gould's B. New Guin. pt. XXIII pl. 1 (1887). 
Mas. Mari Parotiae sexpennis (Bodd.) simillimus, sed scuto pectorali magis aurato et minus viridi nitenti, occipitis crista unicolori caeruleo-chalybaea, violaceonitenti, minime vel vix viridi in medio nitenti, seuto frontali argenteo diverse formato et cauda breviori ristinguendus.

Foem. Foeminae P. sexpennis simitis, sed colore brunneo corporis supra magis castanco, gastraeo magis rufescente, subalaribus olscure castaneis et cauda breviori iversa.

Mas. Long. tot. $0^{\mathrm{m}}, 330$; al. $0^{\mathrm{m}}, 156$; caud. $\left(\mathrm{(}^{\mathrm{m}}, 127\right.$; rostri $0^{\mathrm{m}}, 029$; tarsi $0^{\mathrm{m}}, 054$.

Foem. Long. tot. $0^{\mathrm{m}}, 251$; alae $0^{\mathrm{m}}, 154$; caud. $0^{\mathrm{m}}, 104$; rostri $0^{\mathrm{m}}, 027$; tarsi $0^{\mathrm{m}}, 051$.

$H a b$. in Papuasia - in Nova Guinea meridionali-orientali, Montibus Astrolabii (Hunstein, Forbes).

Questa specie rappresenta nella Nuova Guinea meridionale-orientale la $P$. sexpennis della Nuova Guinea settentrionale-occidentale, e ne differisce pei caratteri sopra indicati. Tra le differenze del maschio vi è anche quella dello scudo frontale argentino,

quale non è largo, ma stretto, formato da piume gli apici delle quali convergono verso il mezzo in una linea diretta dall'avanti all'indietro; inoltre quello scudo si restringe e si prolunga posteriormente, ove passa nel colore bruno-olivaceo scuro; finalmente le parti superiori mancano della tinta rosso-vinacea vellutata, ed hanno una tinta più pallida.

A quanto pare le femmine delle due specie differiscono fra loro più dei rispettivi maschi.

Le dimensioni sopraindicate sono quelle date dallo Sharpe. Il Meyer invece då dimensioni molto minori per questa specie:

†

al.

caud. . $\quad 0^{\mathrm{m}}, 084-0^{\mathrm{m}}, 088$

rostri cul.

tarsi

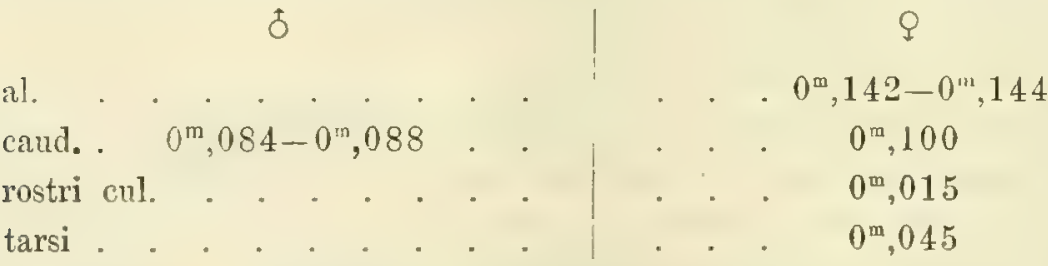

(698) Lophorhina superba (Penn.).

Lophorina superba, Eudes-Deslongch., Ann. Mus, d'Hist. Nat. Caen, I, p. 37 (1880), - Guillem., P. Z. S. 1885, p. 647 (Arpk).

Lophorhina atra, Cory, Beaut. and Cur. Birds, pt. VI (1883). - DHamonv., Butl. Soc. Zool. de Fr. 1886, p. 510.

Paradisea atra, Musschenbr., Dagboek, pp. 193, 226 (1883). - Rosenb., Mitth. Orn. Ver. Wien, 1885, p. 40.

\section{Sp. 1139 (698 bis) Lophorina minor, RaMSAY.}

Lophorhina superba minor, Ramsay, Pr. Linn. Soc. N. S. W. X, p. 242 (1885).

Lophorhina minor, Finsch et Meyer, Zeitschr. f. ges. Orn. II, p. 376, pl. 17 (1885). - Iid., Ibis, 1886, p. 24\%. - Meyer, Zeitschr. fo ges. Orn. III, p. 1S0, cum fig. (1886). -- D'Hamonv., Bull. Soc. Zool. de Fr. 1886, pp. 508, 510. - Sharpe, Nature, vol. 34, p. 340 (1886). - Id., Gould's B. New Guin. pt. XXIV, pl. 1 (1888).

Mas. Mari L. superbae (Penn.) similis, sed minor, et plumis mediis scuti pec[276] 
toralis in medio macula nigro-velutina notatis, plumis nasalibus ercctis (nec bipartitis?), plumis gulae longioribus (?), scuto nitidissimo pilei postice violaceo nitente et praesertim pallio muchali postice in medio profunde diviso, distinguendus.

Foem. Foeminae L. superbae similis, sed supra pallidior, olivaceo-brunnea et tuenia superciliari variegata, in occipite confluente, distinguenda.

Mas. Long. tot. $0^{\mathrm{m}}, 217$; al. $0^{\mathrm{m}}, 138$; caud. $0^{\mathrm{m}}, 100$; rostri $0^{\mathrm{m}}, 027$; tarsi $0^{\mathrm{m}}, 033$ (Ramsay).

Foem. Long. tot. $0^{\mathrm{m}}, 220$; al. $0^{\mathrm{m}}, 120$; caud. $0^{\mathrm{m}}, 080$; rostri $0^{\mathrm{m}}, 021$; tarsi $0^{m}, 028$ (Meyer).

Hab. in Papuasia - Nova Guinea meridionali-orientali, in Montibus Astrolabii (Hunstein).

Questa specie è la rappresentante meridionale-orientale della $L$. superba, e si distingue pei caratteri sopraindicati. Essa fu scoperta, a quanto pare, dall'Hunstein: il Ramsay ne descrisse il maschio, i caratteri del quale furono indicati anche meglio dal Meyer; la femmina fu descritta dal Finsch e dal Meyer.

\section{(699) Paradigalla carunculata, Less.}

Paradigalla carunculata, Eudes-Deslongch., Ann. Mus. d'Hist. Nat. Caen, I, p. 20 (1880). Guillem., P. Z. S. 1885, p. 151 . - D'Hamonv., Bull. Soc. Zool. de Fr. 1886, p. 509.

Astrapia carunculata, Mlusschenbr., Dagboek, pp. 194, 227 (1883). - Rosenb., Mittb. Orn. Ver. Wien, 1885, p. 40.

Il Guillemard, sulle indicazioni del Laglaize, dà il colore delle caruncole diversamente da quello indicato dal Beccari, le cui osservazioni furono fatte su esemplari da lui uccisi, e sono perciò più attendibili.

\section{(700) Astrapia nigra (GM.).}

Astrapia nigra, Fudes-Deslongch., Ann. Mus, d'Hist. Nat, Caen, I, p. 18 (1880). - Cory, Beaut. and Cur. Birds, pt. IV (1882). - Musschenbr., Dagboek. pp. 194, 227 (1883). - Rosenb., Mitth. Orn. Ver. Wien, 1885, p. 40. - Guillem., P. Z. S. 1885, p. 648 (Arfak). - D'Hamonv., Bull. Soc. Zool. de Fr. 1886, p. :09.

Gen. Astrarchia, Meyer.

Typus:

Astrarchia, Meyer, Zeitschr. f. ges. Orn. 1885, p. $378 \ldots . . . .$. A. stephaniae, Finsch.

\section{Sp. 1140 ( 700 bis) Astrarchia stephaniae, FinsCH.}

Astrarchia stephaniae, Finsch et Neyer, Zeitschr. f. ges. Orn. 1885, p. 378, Taf. XVIII. - Iid., Ibis, 1886, p. 245. - D'Hamonv., Bull. Soc. Zool. de Fr. 1886, pp. 505, 509. - Meyer, Journ. f. Orn. 1889, p. 321 (१).

Pileo nitide caeruleo et viridi-violaceo, fronte, loris et genis nitide viridibus, frontis plumis velutinis, erectis; capitis lateribus plumis longis, velutinis, viridibus, purpureo et cupreo-violaceo (nitentibus?) ornatis; scapularibus, interscapulio, dorso, uropygio, axillaribus et hypochondriis nigro-velutinis, sub quandam lucem olivaceo nitentibus; supracaudalibus nigro-velutinis; gula, gutture et collo antico aeneo viridibus, sub quandam lucem caerulescentibus, colli lateribus plumis longis ornatis; fascia pectorali lata nigra, sub quandam lucem olivaceo et litaceo nitente, fascia nitidissime 
cuprea cincta; corpore subtus obscure cupreo-velutino, sub quandam lucem viridi; crisso et subcaudalibus nigro-violaceis; tibiis violaceo-nigris; alis et alarum tectricibus nigris, plus minusve purpurco nitentibus; subalarbus violaceo marginatis; rectricibus duabus intermediis longissimis, naviculiformibus, superne concavis, nitide roseo-violaceis, subtus nigris; rectricibus lateralibus brevibus, superne concavis, supra et subtus nigris; rectricum duarum intermediarum scapis supra ad basin albis; rostro nigro; pedibus nigris; iride nigra (Finsch et Meyer).

Long. tot. $0^{\mathrm{m}}, 840$; al. $0^{\mathrm{m}}, 156$; caud. $0^{\mathrm{m}}, 640$; rostri $0^{\mathrm{m}}, 025$; tarsi $0^{\mathrm{m}}, 041$.

Foem. Minor; capite et collo nigro-coracinis; nucha et corpore supra nitente nigris, plumarum marginibus obscure brunneis; alis caudaque gradata nitente vio7ascenti nigris, sub quandam lucem transfasciolatis, subtus fuscis; corpore subtus, suba7aribus et subcaudalibus nigris, brunnescenti transfasciolatis; rostro pedibusque nigris. (Meyer).

Long. tot. $0^{\mathrm{m}}, 520$; al. $0^{\mathrm{m}}, 149$; caud. $0^{\mathrm{m}}, 340$; rostri $0^{\mathrm{m}}, 025$; tarsi $0^{\mathrm{m}}, 041$.

Hab. in Papuasia - Nova Guinea meridionali-orientali, in Montibus Hufeisen (Hunstein), in Montibus Owen-Stanley (Goodwin).

Questa specie evidentemente è affine alla Astrapia nigra, dalla quale è stata separata genericamente per le timoniere mediane verso l'apice concave, per le timoniere laterali molto più brevi, per la mancanza delle lunghe piume del mento, per la minore lunghezza delle piume laterali della testa e per altre differenze.

Nella femmina le due timoniere mediane non sono verso l'apice concave e rivolte all'insù, ma al tutto piane; esse superano le penultime di 15 centimetri, e queste le seguenti di 6 centimetri; la parte visibile dello stelo delle due timoniere mediane non è bianca, come nel maschio, ma nera; soltanto la parte nascosta è bianca; le timoniere e le remiganti hanno fascie trasversali rilevate.

Il maschio e la femmina di questa bellissima specie si conservano nel Museo di Dresda.

Questa splendida specie fu dedicata all'arciduchessa Stefania, vedova dell'infelice arciduca Rodolfo, Principe ereditario d'Austria.

(701) Epimachus speciosus (Bodd.).

Epimachus speciosus, Cory, Beaut. and Cur. Birds, pt. V (testo) (1883). - Guillew., P. Z. S. 1885, p. 648 (Arfak). - D’Hamonv., Bull. Soc. Zool. de Fr. 1886, p. 509.

Epimachus magnus, Cory, Beaut. and Cur. Birds, pt. V (1883).

Epimachus maximus, Musschenbr, Daghoek, pp. 201, 231 (1883). - Rosenb., Mitth. Orn. Ver. Wien, 1885, p. 53 .

\section{Sp. 1141 (701 bis) Epimachus macleayae, RAMSAY.}

Epimachus macleayanae (1), Ramsay, Pr. Linn. Suc. x. S. W. ser. 2, vol. II, p. 239 (1887) (ठ). - Meyer, Journ. f. Orn. 1889, p. 322 (Q).

Mas. Capite, gula et corpore toto supra nigro-velutinis et, collo, alis et uropygio exceptis, plumis metallicis, squanatis, caeruleo, viridi et violaceo nitentibus btectis; uropygio et supracandalibus nigro-velutinis, phumarum apicibus caenulcochalybacis; cauda nigra, rectricibus duabus intermediis longis, strictis, nigris, cnemuleo et violaceo nitentibus; pectore et gastraeo reliquo fusco-olivaceis, roseo in- 
dutis; plunis laterum pectoris fere triangularibus, ad apicem latissimis late viridi caeruleo-chalybaeo et violaceo marginatis, plumis inferioribus laterum brevioribus roseo-violaceo marginatis, castanco et violaceo nitentibus; plumis clongatis laterum decompositis et ultra subcaudales attingentibus, pallide brunneis; tibiis nigris; supracaudalibus et cauda subtus nigris; corpore subtus pulchre roseo-violaceo nitente, lateribus in quadam luce roseo-lilacinis; pedibus et rostro nigris (ex Ramsay).

Long. tot. $1^{\mathrm{m}}, 066$; al. $0^{\mathrm{m}}, 183$; caud. $0^{\mathrm{m}}, 696$; rostri culm. $0^{\mathrm{m}}, 081$; tarsi $0^{\mathrm{m}}, 056$.

Foem. Vix minor; supra olivascenti-brunnea; pileo et nucha castaneo-rufis; loris, lateribus capitis et gula nigrescentibus; collo antico, corpore subtus et subalaribus fasciis alternis nigris et brumescenti-albis, abdomine et subcaudabus rufescenti tinetis; alis dorso concoloribus, sub quandam lucem transfasciolatis, subtus fuscis, primariarun mediarum narginibus exterioribus paullum rufescentibus; tibiis fuscis; cauda gradata, olivacea, supra brunnescenti tincta, sub quandam lucem transfasciolata, subtus pallidiore; rostro nigro, pedibus nigrescentibus (Meyer).

Long. tot. $0^{\mathrm{m}}, 660 ;$ al. $0^{\mathrm{m}}, 173-0^{\mathrm{m}}, 175$; caud. $0^{\mathrm{m}}, 360$; rostri $0^{\mathrm{m}}, 088$; tarsi $0^{\mathrm{m}}, 049$.

Hab. in Papuasia - Nova Guinea meridionali-orientali, in Montibus Astrolabii (fide Ramsay, Goodwin).

Il Ramsay ebbe un maschio di questa specie proveniente dai piedi dei Monti dell'Astrolabio; recentemente il Meyer ha avuto dal Goodwin un maschio adulto ed un esemplare ch'egli ha descritto come femmina e che probabilmente provengono dalla stessa regione ove fu raccolto il maschio descritto dal Ramsay.

Il Meyer afferma che la descrizione del maschio data dal Ramsay è insufficiente ed in parte erronea, e quindi dà la seguente descrizione del maschio che riferisco tradotta:

Supra niger, subtis brunneus; plumis capitis squamatis nitide caeruleo-viridibus; plumis menti pilosis, obscure violaceis; gula laete violacea, squamata; plumis cervicis collique lateribus nigris arcte sed nitide caeruleo-viridi, sub quandam lucem violaceo marginatis; dorsi et uropygii plumis nigris, maculis longis squamiformibus, nitentibus, cxeruleo-viriditus apice ornatis; alis nigris, caeruleoviridi, sub quandam lucem violaceo nitentibus, alis subtus nitide griseo-nigris; scapularibus violaceo nitentibus; collo antico et pectore olivaceo-brunneis; pectoris lateribus flabellis latissimis ornatis, plumis posticis longissimis flabelli securiformibus, apice triangularibus, nigris violaceo nitentibus, inferius sensim viridibus, brevioribus sensim brunneis; plumis mediis flabelli superioribus nigris, vio$7 a c e o$ ct viridi nitentibus, fascia late nitide caeruleo-viridi marginatis, inferioribus otivaceo-brumeis, duabus marginibus nitidis ornatis, uno pallide violaceo in viridem vergente, altero roseo-violaceo; plumis anterioribus flabelli brunneis, late roseo-violaceo marginatis et viridi nitentibus; abdomine medio brunneo, obscure viridi nitente, latera versus violaceo nitente; plumis laterum elongatis brunneis, apice pulchre violaceis, longioribus decompositis fulvo-brunneis; cauda supra nigra, rectricibus exterioribus vix nitentibus, mediis duabus velutinis, caeruleo-viridi nitentibus, sub quandam lucem crebre transfusciolatis; supracaudalibus nigris, subcaudalibus fulvo-brunneis; rostro et pedibus nigris. 
Long. tot. $1^{\mathrm{m}}, 000$; al. $0^{\mathrm{m}}, 184-0^{\mathrm{m}}, 186$; caud. $0^{\mathrm{m}}, 710$; rostri culm. $0^{\mathrm{m}}, 091$; tarsi $0^{\mathrm{m}}, 052$.

11 Mejer fa notare che nella descrizione del Ramsay è detto che le piume del sopraccòda sono nere vellutate con gli apici di color azzurro metallico, la quale cosa non è esatta, arendo il Ramsay scambiato le piume del sopraccoda con quelle del groppone; inoltre il Ramsay indica le timoniere mediane larghe 3 pollici e mezzo, invece di 3 centimetri e mezzo; il Ramsay non indica il colore bruno del sottocoda, e finalmente egli dice che l'E. macleayanae è molto diverso dall'E. magnus per la lunghezza della coda, laddove ciò non è.

\section{Sp. 1142 (701 ter) Epimachus meyeri, Finsch.}

Epimachus Meyeri, Finsch, Zeitschr, f. ges. Orn. 1885, p. 380 (Q). - Id., Ibis, 1886, p. 247. D’Hamonv., Bull. Soc. Zool. de Fr. 1886, p. 509. - Meyer, Journ. f. Orn. 1889, p. 323.

Foem. Supra brumescenti-olivacea; capitis plumis obscure fuscis; nufo marginatis; nucha rufescente; loris et capitis lateribus nigrescentibus, clarius variegatis; gutture iisdem coloribus, sed subtititer transfasciato; corpore subtus et subalaribus fasciis alternis nigrescentibus et brunnescenti-albidis ornatis; alis dorso concoloribus, subtus fuscis; cauda olivacea, supra vix brunnescente tincta, subtus pallidiore; rostro et pedibus nigris; iride caerulea (Finsch et Meyer).

Long. tot. circa $0^{\mathrm{m}}, 540-0^{\mathrm{m}}, 550$; al. $0^{\mathrm{m}}, 158-0^{\mathrm{m}}, 160$; caud. $0^{\mathrm{m}}, 320$; rostri $0^{\mathrm{m}}, 073$; tarsi $0^{\mathrm{m}}, 042$.

Hab. in Papuasia - Nova Guinea meridionali-orientali, Montibus Hufeisen dictis (Hunstein).

Secondo il Finsch ed il Meyer la femmina descritta differisce da quella dell' $E$. speciosus pel colore delle parti superiori più olivaceo, per la mancanza del colore rugginoso sulle ali, pel colore bruno-rossigno della testa più vivo ed esteso anche sulla cervice, pel disegno a squame della testa, per le fascie ondulate delle parti inferiori più sottili e per la coda senza tinta rossigna; inoltre il becco è un poco più lungo, ma più compresso e meno arcuato.

Io avera dubitato che il tipo di questa specie fosse la femmina dell'E. macleayae, Ramsay, ma il Meyer afferma che ciò non è; tuttavia non pare che egli sia al tutto sicuro nella sua affermazione, giacchè anch'egli fa notare come non sia impossibile che l'esemplare da lui descritto come femmina dell'E. macleayanae sia invece un maschio giovane e che l'E. meyeri sia fondato sulla femmina di detta specie. Di questa cosa io grandemente sospetto, e se venisse confermata, il nome $E$. meyeri avrebbe la priorità su quello di $E$. macleayue.

(702) Epimachus ellioti, WARD.

Epimachus Ellioti, Eudes-Weslongch., Ann. Mus, d'Hist. Nat. Caen, I, p. 8 (1880). - Cory, Beaut. and ('ur. Birds, pt. V (1883). - Musschenbro, Dagboek, pp. 202, 234 (1883). - Rosenb., Milth. Orn. Ver. Wien, 1885, p. 53. - D'Llamonv., Bull. Soc. Zool. de Fr. 1886, p. 509.

Il Meyer mi scrive di aver ricevuto recentemente un maschio di questa specie (della quale si conosceva soltanto un esemplare), insieme con un maschio di una nuova Craspedophora non ancur descritta (!) e col maschio della Drepanornis bruijni. 
(703) Drepanornis albertisii, ScLat.

Drepanornis albertisii, Kudes-Deslongch., Ann. Mus. d'Hist. Nat. Caen, I, p. 15 (1880, - Cory, Beant. and Cur. Birds, pt. VI (1883). - Musschenhr., Dagboek, pp. 204, 235 (1883). - Guillem., P. Z. S. 1885, p. 649 (Arfak)。 - D’Hamonv., Bull. Soc. Zool. de Fr. 1886, pp. 505, 509.

Epimachus albertisii, Rosenb., Mitth, Orn. Ver, Wien, 1885, p. 54.

\section{SP. 1143 (703 bis) Drepanornis cervinicauda, ScLat.}

Drepanornis D'Albertisii, Ramsay (nec Sclat), Pro Linn. Soc. N. S. W. IV, p. 469 (1879); VIII, pp. 16, 28 (1883) (nido ed uovo).

Drepanornis bruijni, part., Salvad., Orn. Pap. e Mol. II, p. 553 (1881).

Drepanornis albertisii, Sharpe (nec Sclat.), Journ. Linn. Soc., Zool. XVI, p. 445 (Taburi) (1882). - Salvad., Orn. Pap. e Mol. II, p. 552 (1882).

Drepanornis albertisii cervinicauda, Sclat., P. Z. S. 1883, p. 578.

Drepanornis cervinicauda, Sharpe, Gould's B. New Guin. pt. XVIII, pl. 1 (1884). - Meyer et Finsch, Zeitschr. F. ges. Urn. 1885, p. 381, TaF. XIX. - Iid., Ihis, 1886, p. 248, - D'Hamonv. Bull. Soc. Zool. de Fr. 1886, pp. 505, 509.

Drepanornis D. alhertisii, Sclat. similis, sen minor et uropygio caudaque pallidioribus, distinguenda.

Long. tot. $0^{\mathrm{m}}, 320-0^{\mathrm{m}}, 330$; al. $0^{\mathrm{m}}, 150-0^{\mathrm{m}}, 152$; caud. $0^{\mathrm{m}}, 128-0^{\mathrm{m}}, 130$; rostri $0^{\mathrm{m},}, 071-0^{\mathrm{m}}, 077$; tarsi $0^{\mathrm{m}}, 031-0^{\mathrm{m}}, 032$.

Hab. in Papuasia - Nova Guinea meridionali-orientali ad flumen Goldie (Goldie), Taburi (Goldie), in Montibus Hufeisen (Hunstein).

11 Meyer oltre alle differenze sopramenzionate ne indica molte altre minori, e fá notare come le femmine delle due specie differiscano più dei maschi, avendo quella della $D$. cervinicauda il dorso olivaceo, anzichè bruno-rugginoso.

Questa specie fu da prima riferita alla D. albertisii dal Ramsay, dallo Sharpe e da me, sulla fede di quegli autori, non "avendone io veduto alcun esemplare; lo Sharpe, il quale da prima ebbe soltanto la femmina, fece notare com'essa avesse la coda più chiara della $D$. albertisii; poscia essa fu distinta dallo Sclater.

Il Ramsay ha aruto il nido e le uova di questa specie, e descrive il primo come costruito nella biforcatura di un ramo, ove è tesa una sorta di rete intessuta di sottili radici, e sopra la medesima sta il vero nido formato di sottili erbe e poco profondo; le uova grandi $34 \times 25 \mathrm{~mm}$. sono di color giallognolo macchiettate di bruno-rossigno e di grigio-violaceo chiaro, piủ fittamente verso la estremità più grossa.

(704) Drepanornis bruijni, Oust.

Drepanornis bruijnii, Musschenhr., Dagboek, pp. 206, 236 (1883). - Guillem., P. Z. S. 1885, p. 649. - Meyer, Zeitschr. f, ges. Orn. 1885, p. 382. - ld, Ihis, 1886, p. 249. - Sharpe, Gould's B. New Guin. pt. XXI, pl. 2 (1886). - D'Hamonv., Bull. Soc. Zool. de Fr. 1886, pp. 505, 509. - Oust., L.e Naturaliste, 1887, p. 180 (ठ). - Ibis, 1889, p. 583.

Epimachus Bruijnii, Rosenb., Mitth. Orn. Ver. Wien, 1885, p. 54.

Il maschio di questa specie è stato descritto dall'Oustalet nel 1887 e, cosa singolare, sebbene siano corsi quasi tre anni da quella dàta nessuno dei giornali ornitologici ne hanno parlato e neppure se ne trova fatta menzione nel Zoological Record pel 1887 !

Trascrivo qui la descrizione dell'Oustalet, essendo molto difficile di riassumerla in poche parole: 
« Chez cet oiseau, 'deux bandes de plumes veloutées et légèrement érectiles partent $d u$ bec et remontent au-dessus des yeux, comme chez le $D$. Albertisii; mais ces bandes ne sont pas séparées en arrière; elles deviennent confluentes sur le vertex et tendent à se fondre avec une plaque formée de plumes analogues et occupant le sommet de la tête. En outre, ces bandes super-oculaires sont d'un brun pourpré sans reflets métalliques bleues ou verts, comme chez le $D$. Albertisii, tandis qu'en revanche les plumes veloutées qui courrent le menton et les côtés de la tête, en errière de la mandibule inférieure, sont d'un vert bronzé moins uniforme que dans cette dernière espèce, puisqu'au milieu d'elles on voịt ápparaître latéralement deux petites taches, d'un bleu verdâtre métallique et que, sur le côté, on distingue des reflets pourprés.

La plaque mentonnière descend d'ailleurs un peu plus bas que chez le $D$. Albertisii et rencontre, sur le haut de la poitrine, une sorte de fraise formée de plumes décomposées de couleur brune sur la majeure partie de leur longueur et teintées de vert métallique dans leur portion terminale qui est tronquée aussi nettement que si elle àvait été coupée arec des ciseaux. Cette fraise s'allonge un peu de chaque côté en deux touffes d'un brun légèrement glacé de vert olive qui représente les deux grandes touffes pectorales du $D$. Albertisii et qui recouvrent les ornements des flanes. Ceux-ci offrent un tout autre aspect que chez les Drepanornis précédemment connus. Ils consistent en un double croissant de plumes métalliques et veloutées de diverses couleurs. Vers le plis de l'aile ce sont d'abord deux ou trois plumes noires, veloutées, ornées d'une magnifique bordure de couleur cuivre rouge ou plutôt bronze florentin, et rappellant, ảrec des dimensions plus faibles et des dimensions plus réduites les plumes qui ornent les côtes et la gorge de l'Astrapia nigra; de petites plumes noires, veloutées et glacées de violet à l'extrémité leur succèdent et sont suivies à leur tour d'une double rängée de plumes arrondies dont les unes, celles qui confinent au bord de l'aile, sont d'un noir de velours à liséré vert brillant, tandis que les autres, insérées plus en dedans, sont d'un gris cendré, avec bordure d'un vert olive très luisant. Dans la région postérieure de l'abdomen on n'aperçoit ancune trace des touffes qui s'épanouissent de chaque côté du ventre du $D$. Albertisii et qui sont ornées d'une légère bordure violette. Ici toute cette région, de même que la poitrine, est d'un beau gris cendré, et vers le milieu du ventre présente une teinte fauve, avec quelques räies transversales brunes, vestiges de la livrée du jeune âge.

Le manteau est d'un brun notablement plus foncé que chez le D. Albertisii, et, sous un certain jour, paraît légèrement glacé de gris dans la région scapulaire; les ailes elles-mêmes sont plutôt brunes que rousses, la queue est d'une couleur fauve beaucoup moins claire que chez le $D$. Albertisii et chez le $D$. cervinicrudn et les rectrices, de même que les pennes secondaires des ailes présentent, sous une certaine incidence de lumière, de petites raies transversales foncées.

Les pattes sont brunes, avec les ongles noirs et les mandibules, sur la dẻpouille que j'ai sous les yeux, offrent la même teinte cornée que chez le jeune oisean que j'ai incidemment décrit. Enfin, l'espace dénudé qui occupe le côté de la tête est plus vaste, moins irrégulièrement dessiné que chez les $D$. cervinicauda et Albertisii et montre une peau d'un noir livide qui était sans doute perdu d'une teinte bleuâtre plus ou moins accusée chez l'oiseau vivant. 
Les dimensions principales du Drepanornis Bruijni adulte sont les suivantes:

Longueur totale $0^{m}, 350$; longueur de l'aile $0^{m}, 160$; de la queue $0^{m}, 130$; du tarse $0^{\mathrm{m}}, 030$; du doigt medium sans l'ongle $0^{\mathrm{m}}, 030$ (?), du bec (culmen) $0^{\mathrm{m}}, 080$ (?) ».

Il giovane e la femmina della $D$. bruijni si riconoscono facilmente per diversi caratteri : $1^{\circ}$ al becco rossigno-pallido (e non nero) e molto più grosso alla base; $2^{\circ}$ all'area perioculare nuda molto più grande e di forma diversa; $3^{\circ}$ al colore nericcio della testa che tinge alquanto tutte le parti superiori; $4^{\circ}$ alla fascia nera a modo di mustacchio lungo la regione malare; 5 " finalmente alle fascie scure delle parti inferiori più regolari ed estese fino al sottocoda.

Lungh. tot. circa $0^{m}, 304$; al. $0^{m}, 142$; coda $0^{m}, 109$; becco $0^{m}, 068$; tarso $0^{m}, 031$.

Questa specie è propria della Nova Guinea settentrionale ad oriente della baia del Geelwink; il maschio adulto descritto dall'Oustalet proveniva da un punto della costa di rimpetto all'isola Podena, o Padiema.

Nel giornale «The Ibis», 18.89, p. 581, è imenzionata la Drepanomis bruijui come specie dei Monti Arfak, la quale cosa certamente non è esatta.

\section{(705) Craspedophora magnifica (VIEILL.).}

Craspedophora magnifica, Eudes-Deslongch., Ann. Nus. d'Hist. Nat. Caen, I, p. 10 (1880). Guillem., P. Z. S. 1885, p. 650 (Andai). - Meyer, Zeitschr. f. ges. Orn. 1886, p. 36 (Kafu).

Plilornis magnifica, Musschenhr., Dagboek, pp. 199, 231 (1883). - Kosenb., Mitth. Orn. Ver. Wien, 1885 , p. 40.

Ptilorhis magnifica, D'Hamonv, Bull. Soc. Zool. de Fr. 1886, p. 508.

\section{(1020) Craspedophora intercedens (SHARPE;.}

Ptilornis Alberti, part., Musschenbr., Dagboek, pp. 200, 232 (1883). - Rosenb., Mitth. Orn. Ver. Wien, 1885, p. 40.

Craspedophora intercedens, Meyer, Zeitschr. f. ges. Orn. 1885, p. 382. - Id., Ibis, 1886, p. 250.

Ptilorhis intercedens, D'Hamonv., Bull. Soc. Zool. de frr. 1886, pp. 504, 508.

Craspedophora magnifica, Tristr. (nec Vieill.), Ibis, 1889, p. 554 (Milne Bay).

Il Meyer dice che la distinzione di questa specie dalla C. magnifica (Vieill.) è difficile, tuttavia egli l'ammette.

\section{(706) Seleucides nigricans (SHAW).}

Sele ucides alba, Eudes-Deslongch., Ann. Mus. d'Hist. Nat. C'aen, I, p. 13 (1880). - D'Hamonv., Bull. Soc. Zool, de Fr. 1886, p. 509.

Epimachus albus, Musschenbr, Dagboek, pp. 202, 234 (1883). - Rosenh., Mitth. Orn. Ver. Wien, 1885 , p. 53.

Seleucides nigricans, Guillem., P. Z. S. 1885, p. 650 (Salwatti).

Il Guillemard descrive i costumi di un esemplare avuto vivo in Salavatti.

(707) Semioptera wallacei (G. R. GR.).

Epimachus Wallacei, Musschenbr., Dagboek, pp. 203, 235 (1883). -- Mosenb., Mith, Orn. Ver. Wien, 1885, p. 53.

Semioptera Wallacei, Eudes-Deslongch., Ann. Mus. d'Hist. Nat. Caen, I, p. 38 (1880). - Guillem. P. Z. S. 1885, p. 574 (Batchian). - D'Hamonv., Bull. Soc. Zool. de Fr. 1886, p. 510.

a. Semioptera wallacei, var. halmaherae, SALVAD.

Epimachus Wallacei, var. Halmaheirae, Musschenbr, Dagboek, pp. 204, 235 (1883). 
(708) Paradisea minor, SHaw.

Paradisea minor, Eudes-Deslongch., Ann. Mus, d'Hist. Mat. Caen, I. p. 22 (1880). - Musschenbr., Dagboek, pp. 185, 220 (1883). - Rosenb., Mitth. Orn. Ver. Wien, 1885, p. 30. - Guillem., P. Z. S. 1885, p. 651 (Jobi, Dorei, Mysol). - D'Hamonv., Bull. Soc. Zool. de Fr. 1886, pp. 506, 509.

Il Guillemard parla dei costumi di quattro esemplari avuti vivi, tre dei quali furono deposti nel Giardino Zoologico di Londra.

Il Musschenbroek (Dagboek, pp. 186, 221) menziona una Paradisea minor var. albescens, fondata sopra un uccello tutto bianco, menzionato dal Valentyn, III, p. 310, per averne inteso parlare, ma che probabilmente non è mai esistito, e sul quale si fondano la Paradisea candida, Forster, e la $P$. alba, Gm.

\section{Sp. 1144 ( $708^{\text {bis }}$ ) Paradisea finschi, Meyer.}

Paradisea Finschi, Meyer, Zeitschr. f. ges. Orn. 1885, p. 383; 1886, p. 36. - Id., Ibis, 1886, p. 250. - D’Hamonv. Bull. Soc. Zool. de Fr. 1886, pp. 507, 509.

Mas. Paradisea P. minori similis, sed minor et dorso, uropygio et corpore subtus brunnescentibus (Meyer).

Long. rostri $0^{\mathrm{m}}, 031$; tarsi $0^{\mathrm{m}}, 040$.

Hab. in Papuasia - Nova Guinea septentrionali-orientali, Karan (Finsch).

Dice il Finsch: « io potei avere soltanto pelli imperfette di questa specie dagli indigeni della costa settentrionale-orientale (Terra dell'Imperatore Guglielmo) nella long. or. $142^{\circ} 30^{\prime}$, circa 60 miglia ad occidente (?) dell'Isola D'Urville.... Tutte le pelli di questa località hanno piccole dimınsioni. »

L'unica pelle preparata dagli indigeni, che è il tipo di questa specie, differisce secondo il Meyer, dalla P. minor per avere le parti superiori non di color castagno puro, ma di un bruno più pallido, e le parti inferiori non di color bruno-castagno vinaceo, ma di un bruno rossigno; inoltre la $P$. finschi arrebbe il becco più assottigliato ed allungato.

\section{Sp. 1145 (708 $^{\text {ter }}$ ) Paradisea guilielmi II, CAB.}

Paradisea Guilielmi, Cab., Journ. f. Orn. 1888, p. 119, n. 1.

Paradisea Guilielmi II, Cab., Journ. f. Urn. 1889, Tab. I ( $\delta$ et $q$ ).

Paradisea pileo toto, capitis collique lateribus et gutture a mento ad pectus nitide viridibus; mucha, dorso et alarum tectricibus minoribus pallide flavis; paracerco albo, intus parte basali flavo, singulis plumis laxis, radiis longissimis; alis, uropygio caudaque obscure castaneis; pectore ventrisque lateribus chocolatinis; ventre imo, crisso et tibiis in nigrum vergentibus (Cabanis).

Foem. Pileo, capitis, collique lateribus et gutture a mento ad pectus castaneovinaceis, alae, dorso medio, uropygio et caudae concoloribus; occipite, collo postico, interscapulio et tectricibus alarum minoribus flavo-stramineis; abdomine castaneovinacro (ex Icone foeminae).

Hab. in Papuasia - Nova Guinea orientali-septentrionali, Kaiser Wilhelm's Land (Cabanis).

Il Cabanis non dà i caratteri differenziali di questa specie; egli aveva promesso $\lceil 284\rceil$ 
di pubblicare ulteriori osservazioni intorno a questa specie, ma finora non l'ha fatto. Giudicando dalla descrizione, parrebbe che questa specie sia distinta dalla $P$. minor principalmente per arere tutto il pileo, i lati della testa e del collo, e la gola dal mento al petto di color verde splendente, e pel petto e pei fianchi di color castagno, laddove l'addome, il sottocoda e le tibie sono nereggianti.

\section{Sp. 1146 (7084) Paradisea augustae victoriae, CAB.}

Paradisea Augustae Victoriae, Cab., Journ. f. Orn. 1888, p. 119, n. 2. - Ibis, 1889, p. 583. Cab., Journ. f. Orn. 1889, Tab. II (ô, Q).

Paradisea fronte guttureque nitide viridibus, mento nigro; pectore plumis veIutinis atropurpureis; gastraeo toto reliquo vinaceo; paracerco laete rufo-aurantio; capite supra nucháque flavis; notaeo reliquo cum caudae tectricibus medialiter flavis, lateraliter cum alarum tectricibus flavo-olivaceo lavatis; alis caudaque obsolete castaneis (Cabanis).

Hab. in Papuasia - Nova Guinea orientali-septentrionali, Kaiser Willhelm's Land (Cabanis).

Questa specie, che è stata inviata al Museo di Berlino, come proveniente dalla stessa regione della precedente (!), si distingue dalla $P$. minor per le lunghe piume dei fianchi di color rossigno-arancio, pel color giallo delle parti superiori mediane esteso fino al sopraccoda e pel petto di color atro-purpureo.

(709) Paradisea apoda, Linn.

Paradisea apoda, Fudes-Deslongch., Ann. Mus. d'Hist. Nat. Caen, I, p. 20 (1880). - Salvad., Voy. Chall., Birds, p. 81 (1881). - Cory, Beaut. and Cur. Birds, pt. VII (1883). - Musschenbr., Dagboek, pp. 176, 219 (1883). - Neyer, Zeitschr. f. ges. Orn. I, p. 293, Taf. XVII, f. 2 (ovum) (1884). Rosenb., Mitth. Orn. Ver. Wien. 1885. p. 17 (partim). - Guillem., P. Z. S. 1885, p. 652 (Aru). D’Hamonv., Bull. Soc. Zool. de Fr. 1886, p. 509. - Bartl, P. Z. S. 1887, p. 392.

11 Bartlett (l.c.) discorre della muta di un maschio vivo affidato alle sue cure.

(710) Paradisea novae guineae, D'Alb. et SAlvad.

Paradisea apoda var. Novae Guineae, Musschenbr,, Dagboek, pp. 182, 220 (1883).

Paradisea apoda, part., Rosenb., Mitth. Orn. Ver. Wien, 1885, p. 17 (partim).

(711) Paradisea raggiana, Sclat.

Paradisea raggiana, Eudes-Deslongch., Ann. Mus. d'Hist. Nat. Caen, I, p. 24(1880). - Musschenbr., Dagboek, pp. 184, 220 (1883). - Ramsay, Pr. Linn. Soc. N. S. W. VII, p. 26 (1883) (nido ed uova). - Rosenb, Mitth. Orn. Ver, Wien, 1885, p. 30. - Chalm. and Wyatt, Work and Adent. in New Guin. (Ibis, 1885, p. 463). - Finsch et Meyer, Zeitschr. f. ges, Orn. 1885, p. 384. - Iid., Ibis, 1886, p. 251. - Sharpe, Nature, vol. 34, p. 340 (1886). - D'Hamonv., Bull. Soc. Zool. de Fr. 1886, pp. 507, 509. - Tristr., Ibis, 1889, p. 553 (Cast ('ape).

Secondo l'Hunstein, questa specie comincia ad incontrarsi nell'interno a 15 miglia da Port Moresby, ma non si trova nei Monti Ferro di Cavallo; essa si estende all'Isola Basilisk e verso occidente alla Baja Bentley.

Il Finsch ed il Meyer fanno notare alcune differenze che presentano gli esemplari della Baja Milne, i quali arrebbero lo scudo pettorale bruno-violaceo più oscuro e le parti sottostanti pure più oscure degli esemplari dei Monti dell'Astrolabio; inoltre in questi le piume verdi della gola avrebbero riflessi gialli, mentre 1 primi avrebbero quei riflessi azzurrognoli. 


\section{Sp. 1147 (711 bis) Paradisea decora, SALv. et Godm.}

Paradisea decora, Salv, et Godm., Ibis, 1883, p. 131 (January); p. 202, pl. VIII. - Sharpe, Gould's B. New Guin. pt. XX, pl. 1 (1885), - N'Hamonv., Bull. Soc. Zool. de Fr. 1886, pp. 507, 509. - Tristr., lbis, 1889 , p. 553.

Paradisea Susannae, Ramsay, Pr. Linn. Soc. N. S. W. VIII, p. 21 (31st Jan. 1883, published 19th June 1883). - Salvad, Ibis, 1884, p. 354.

Supra sericeo-straminea, alis et cauda fuscis, illarum tectricibus stramineo lavatis; rectricibus mediis elongatis filiformibus, ramis ad medium evanescentibus, sicut in P. apoda; fronte angusta et gula velutino-viridescenti nitentibus, mento sub certa luce obscuriore; subtus lilacino-vinacea, pectore saturatiore, abdomine medio albicantiore; plumis hypochondriacis posticis ruberrimis, apicibus canescentibus, ramis valde distantibus sicut in $\mathbf{P}$. sanguinea, anticis brevibus, apicibus saturatissime rubro vinaceis; rostro plumbeo ad apicem allicante; pedibus pallide plumbeis; iride flava.

Long. tot. $0^{\mathrm{m}}, 355$; al. $0^{\mathrm{m}}, 177$; caud. rectr. lateral. $0^{\mathrm{m}}, 152$; rectr. med. elong. $0^{\mathrm{m}}, 457$; rostri a rictu $0^{\mathrm{m}}, 040$; tarsi $0^{\mathrm{m}}, 045$.

Foem. Inornata, supra olivaceo-fusca, stramineo tincta; gulae saturate brunnea; subtus rufescenti-fusca, pectore et hypochondriis anticis fusco irroratis; caudae rectricibus duabus mediis angustis et acutis, retiquis paullo brevioribus.

Hab. in Papuasia - Ins. Fergusson (D'Entrecasteaux archipelago) (Goldie, Thomson).

Questa specie somiglia più che ad altra alla $P$. raggiana, avendo come questa le piume dei fianchi rosse, ma ne differisce principalmente per avere il dorso giallo ed il petto grigio-vinaceo e per mancare del collare giallo separante il verde della gola dal grigio-vinaceo del petto. La femmina si distingue facilmente pel colore brunoolivaceo e non castagno delle parti superiori.

La $P$. decora fu scoperta dal Goldie nell'Isola Fergusson (una delle Isole d'Entrecasteaux), ove, come scrive il Goldie, si trovano anche la Manucodia comriei e l'Otidiphaps insularis. Essa vive sui monti ad una considerevole elevazione; il suo grido somiglia a quello della $P$. raggiana; anche $\mathrm{i}$ suoi movimenti sono simili; le femmine vivono appartate dai maschi.

\section{(712) Uranornis rubra (LACÉP.).}

Paradisea sanguinea, Eudes-Ileslongch., Ann. Nus. d'Hist. Nat. Caen, I, p. 25 (1880). - Cory, Beaut. and Cur. Birds, pt. VI (1883). - Musschenbr., Dagboek, pp. 188, 221 (1883). - Rosenb., Mitth. Orn. Ver. Wien, 1885, p. 31.

Paradisea rubra, Jehrk., Journ. f. Orn. 1885, p. 34 (Waigeu). - Guillem., P. Z. S. 1885, p. 653 (Waigiou, Batanta). -.- D'Hamonr., Bull. Soc. Zool, de Fr. I, pp. 506, 509.

\section{(713) Diphyllodes magnifica (Penn.).}

Diphyllodes magnifica, Eudes-Deslongch., Ann. Mus. d'Hist. Kat. Caen, I, p. 32 (1880). - Guillem., P. Z. S. 1885 , p. 654 Salwatti

Paradisea speciosa, Musschenbr., Dagboek, pp. 190, 222 (1883). -- D'Hamonv., Bull. Soc. Zool. de Fr. 1886, p. 510.

Diphyllodes speciosa, Mosenh., Mitth. Orn. Ver. Wien, 1885, p. 31 
Se. 1148 (713 bis) Diphyllodes jobiensis, MEYer.

Paradisea speciosa, part., Schleg., Ned. Tijdschr. Dierk. IV, pp. 17, 50 (1871). -. Rosenb. (nec Bodd.), Reist. naar de Geelvinkb. p. 56 (1875), - Id, Malny. Archip. p. 557 (1879 _ - Id., Mitth. 0rn. Ver. Wien, 1885, p. 31.

Diphyllodes chrysoptera, Meyer (nec Gould), Mitth. Zoul. Mus, Dresd, I, p. 6 (nota) (1875). Becc., Ann. Mus. Civ. Gen. VII, p. 710 (1875). - Salvad., lbid. p. 971 (1875); IX, p. 192, n. 21 (1876). - Sharpe, Cat. B. III, p. 175 (partim) (1877, - Eudes-Deslongch., Ann. Mus. d'Hist. Nat. Caen, I, p. 33 (1880). - Salvad., Orn. Pap. e Mol. II, p. 641 (partim) (1881). - Guillem., P. 7. S. 1885, p. 654 (Jobi).

Paradisea chrysoptera, Musschenbr., Dagboek, pp. 190, 222 (1883).

Diphyllodes jobiensis, Meyer, Zeitschr. f. ges. Orn. 1885, p. 388. - Id., Ibis, 1886, p. 256. -D'Hamonv, Bull. Soc. Zool. de Fr. 1886, p. 510.

Dice il Meyer che il maschio di questa specie differisce da quello della $D$. magnifica, oltrechè pel colore giallo-ar.nciato delle ali, anche pei lievi riflessi bronzati e bruni dell'addome, ma nella pagina seguente lo stesso Meyer indica l'addome di color braunlichviolett (!). La lunghezza dell'ala varia da 113 a $114 \mathrm{~m}$. e quella della coda da 48 a 50 mm.

La femmina è notevolmente diversa da quella della $D$. magnifica, arendo le parti superiori di color grigio-bruniccio, invece di bruno-olivaceo, tranne $\mathbf{i}$ margini $\mathrm{d}_{\mathrm{i}}$ alcune remiganti secondarie, che sono più distintamente bruni; le parti inferiori sono più biancheggianti. Ala $113 \mathrm{~mm}$, coda $65 \mathrm{~mm}$.

Dopochè lo Sharpe ha mostrato che il nome $D$. chrysoptera spetta agli esemplari della Nuova Guinea meridionale-orientale, il Meyer ha confrontato con questi quelli di Jobi e li ha trovato differenti specificamente.

\section{(714) Diphyllodes chrysoptera, Goold.}

Diphyllodes chrysoptera, Gould, MS, - Elliot, Mon. Parad. text pl. 12 (1873).

Diphyllodes speciosus var, chrysopterus, Elliot, Mon. Parad. text pl. 13 (1873).

Diphyllodes chrysoptera, Elliot, Mon. Parad, pl. 13 (1873). - Gould, B. New Guin. pt. II, pl. 4 (1876). - Sharpe, Gat. B. III, p. 175 (partim) (1877). - Id., Journ. Linn. Soc., Zool. XVI, p. 443 (Choqueri) (1882). - Salvad., Orn. Pap. e Mol. III. p. 553 (1882), - Ramsay, Pr. Linn. Soc. N. S. W. VIII, p. 15 (1883). - Neyer, Zeilschr. f. ges. Urn. II, p. 387 (1885). - Id., Ibis, 1886, p. 255. - D’Hamonv. Bull. Soc. Zool. de Fr. 1886, p.'510.

Mas. Mari D. magnificae similis, sed alis supra pulchre aurantiacis et interscapulio brunnescenti-sanguineo diversus.

Long. al. $0^{\mathrm{m}}, 105-0^{\mathrm{m}}, 108$; caud. $0^{\mathrm{m}}, 038-0^{\mathrm{m}}, 040$.

Fem. long. al. $0^{\mathrm{m}}, 103-0^{\mathrm{m}}, 112$; caud. $0^{\mathrm{m}}, 056-0^{\mathrm{m}}, 060$.

Hab. in Papuasia — Nova Guinea meridionali-orientali, Choqueri (Goldie).

Dice il Meyer: Questa specie differisce dalla D. magnifica non solo pel bel colore giallo-arancio delle ali, ma anche pel colore rossigno-sangue delle piume interscapolari, le quali nella $D$. magnifica sono di color bruno-castagno, o rosso-bruno scuro; inoltre essa differisce pel colore bruno-arancio, e non bruniccio soltanto, della testa, pel bel colore (tinta?) porporino dell'addome e finalmente per lo scudo pettorale verde-azzurrognolo, anzichè verde erba.

Anche le femmine della $D$. chrysoptera sono differenti avendo la testa, il groppone ed il sottocoda di color più bruno; noteroli sono le piume del sincipite, lo quali 
hanno una tinta bruno-arancio, che ricorda la tinta del maschio. Rispetto alle dimensioni la $D$. chrysoptera è alquanto più piccola.

Questa specie fu descritta dal Gould sopra esemplari d'ignota provenienza; il Meyer ed il Beccari credettero che alla medesima dovessero essere riferiti gli esemplari di Jobi; io seguii la loro opinione, ma feci notare come questi differissero dalle figure dell"Elliot e del Gould per avere il sopraccoda bruno-nerastro ed il groppone bruno-aranciato, laddove in quelle figure il groppone ed il sopraccoda sono di colore nerastro uniforme. Lo Sharpe, confrontando esemplari di Coqueri coi tipi del Gould, dimostrò la loro identità, e che perciò alla specie della Nuova Guinea meridionaleorientale dovera essere serbato il nome di $D$. chrysoptera, e che non era improbabile che gli esemplari di Jobi appartenessero ad una specie distinta.

Intorno ai costumi di questo uccello scrive il Goldie che esso si trova nelle regioni fittamente boscose, alla imboccatura dei burroni, o sulle ripe, ove esso prepara uno spazio di terreno lungo circa sette piedi e largo 4, strappando tutte le foglie ed i sottili rami dei cespugli, lasciando soltanto i rami più grossi; quel luogo diventa il campo del sollazzo, ov'esso balla e saltella. Fuori del tempo della cova sta sugli alberi più alti. Si nutre di semi.

\section{Sp. 1149 ( $714^{\text {bis }}$ Diphyllodes hunsteini, MeYer.}

Diphyllodes Hunsteini, Meyer, Zeitschr. f. ges. Orn. 1885, p. 389. - Id., Ibis, 1886, p. 256 D’Hamonv., Bull. Soc. Zool. de Fr. 1886, p. 510.

Diphyllodes chrysoptera, Rosenb. (nec Gould), Mittb. Orn. Ver. Wien, 1885, p. 298.

Mas. Mari D. chrysopterae, Gould, similis, sed alis supra aurantiaco-miniatis diversus.

Long. tot. circa $0^{\mathrm{m}}, 185 ;$ al. $0^{\mathrm{m}}, 111-0^{\mathrm{m}}, 112$; caud. $0^{\mathrm{m}}, 053$; rostri culm. a plumis frontalibus $0^{\mathrm{m}}, 0185$; tarsi $0^{\mathrm{m}}, 029$.

Hab. in Papuasia - Nova Guinea meridionali-orientali, Montibus Owen Stanley (Hunstein).

Dice il Meyer che il tipo di questa specie è un maschio non adulto, ma siffattamente diverso dalla $D$. chrysoptera da non poterne attribuire le differenze allo stadio in cui esso si trova. Il colore delle ali è molto più splendido che non nella $D$. chrysoptera, le piume nasali sono più lunghe ed il groppone maggiormente tinto di color bruno rossigno, l'addome non è violaceo, ma, come quello della $D$. magnifica, è tinto di verdognolo sui fianchi, lo scudo pettorale è di un verde-erba, anzichè verde-azzurrognolo ed il vessillo interno delle remiganti inferiormente più rossigno che non nella $D$. chrysoptera.

\section{(715) Schlegelia respublica (BP.).}

Schlegelia Wilsoni, Eudes-Deslongch., Ann. Mus, d'Hist. Nat. Caen, I, p. 34 (1880).

Diphyllodes respublica, Cory, Beaut, and Cur. Birds, pt. III (1881).

Paradisea Wilsoni, Musschenbr., Dagboek, pp. 189, 221 (1883).

Diphyllodes wilsoni, Nehrk., Journ.f, Orn. 1885, p. 34 (Waigeu). - Guillem., P. Z. S. 1885, p. 654 (Batanta, Waigiou), - D'Hamonv., Bull. Soc. Zool. de Fr. 1886, p. 510.

Paradisea calva, Rosenb., Mitth. Orn. Ver. Wien, 1885, p. 31.

\section{(716) Rhipidornis guglielmi III (MusscH.).}

Rhipidornis respublica, Eudes-Deslongcb. (nec Bp.), Ann. Mus. d'Hist. Nat. Caen, I, p. 30 (1880! Rhipidornis guglielmi tertii, Sclat., P. Z. S. 1883, p.292(Waigeu). - Meyer, P. Z. S. 1886, p. 297. 
Paradisea Guglielmi III, Musschenbr., Dagboek, pp. 190, 223 (1883). - Rosenb., Mitth. Orn. Ver. Wien, 1885 , p. 31.

Diphyllodes Gulielmi III, D Hamonv。, Bull. Soc。 Zool. de Fr. 1886, p. 510.

Ai tre esemplari conosciuti di questa specie se ne sono aggiunti altri due. Nel 1883 lo Sclater mustrò alla Società Zoologica di Londra, da parte di Mr. Whitely, un esemplare di questo uccello; esso faceva parte di una collezione fatta in Waigiu, ed ora si conserva nel Museo Britannico. Poscia il Meyer $(l . c$.$) ha fatto menzione$ di un altro esemplare maschio (il quarto conosciuto) acquistato da un negoziante di Amsterdam; gli altri uccelli che con esso si trovavano erano specie proprie della Nuova Guinea, e non di Waigiu, e però il Meyer crede fosse esatta la supposizione del Beccari (Ann. Mus. Civ. Gen. VII, p. 710) che la Rhipidornis guglielmi III si trovi nella Nuova Guinea settentrionale-occidentale ed in Salavatty.

\section{(717) Gicinnurus regius (Linn.).}

Cicinnurus regius, Eudes-Deslongch., Ann. Nus, d'Hist. Nat. Caen, I, p. 28 (1880). - Salvad., Voy. Chall., Birds, p. 81 (1881). - Cory, Beaut. and Cur. Birds, pt. II (1881). - - Sharpe, Juurn. Linn. Soc., Zool. XVI, p. 444 (Taburi, East Cape) (1882). - Meyer, Zeitschr. f. ges. Orn. I, p. 293 (1884). - Guillem., P. Z. S. 1885, p. 555. - Meyer, Zeitschr. f. ges. Orn. 1886, p. 34 (Kafu). - D'Hamonv., Bull. Soc. Zool. de Fr. 1886, p. 509. - Tristr., Ibis, 1889, p. 554.

Paradisea (Cicinnurus) regius, Musschenbr., Dogboek, pp. 192, 224 (1883).

Paradisea regia, Rosenb., Nitth. Orn. Ver. Wien, 1885, p. 39.

Il Guillemard assicura di arer visto un esemplare di questa specie in Batanta. ove finora non era stata trovata.

Il Meyer nota alcune differenze in un esemplare di Kafu, somigliante a quelli di Jobi, ma di un giallo-rosso vivo.

Gen. PARAdisornis, Meyer.

Paradisornis, Meyer, Zeitschr. f. ges. Orn. 1885, p. 385 . . . . . . P. rudolphi, Meyer.

\section{Sp. 1150 (717 bis) Paradisornis rudolphi, Finsch.}

Paradisornis Rudolphi, Finsch, Zeitschr. f. ges. Orn. 1885, p. 385, Taf. XX. - Finsch u. Meyer, op. cit. 1886, p. 29. - Finsch, Ibis, 1886, p. 252. pl. VII. - Sharpe, Nature, vol. 34, p. 340 (1886). - D'Hamonv., Bull. Soc. Zool. de Fr. 1886, p. 509, - Ramsay, Pr. Linn. Sọc. N. S. W. ser. 2, rol. II, p. 25 (1887).

Sincipite, loris, genis, mento, gula et gutture plumis velutinis, nigris, nitide obscure flavo-virescentibus; regione postoculari nuda; oculis supra et subtus plumis nitide albis, longis, sericeis ornatis; occipite obscure purpureo-castaneo nitente; mucha et interscapulio nitide nigrescentibus, plumis basin versus cinerascentibus, medio cyaneis, apicibus nitide nigrescentibus; dorso ct uropygio caenulescenti-nigro variegatis; cauda supra caerulescente; alis supra caerulescenti-cyaneis, remigum pogoniis internis vigris, alarum tectricibus cyaneis, tertiariis interne clarius cyaneo marginatis; pectoris plumis longis nigrescentibus, abdomen versus virescenti-cueruleo nitentibus; abdomine et tibiis nigris, nitentibus; cauda subtus cyanescente, subcaudalibus virescenti-nigricantibus; alis subtus fuscis, remigum primariorum marginibus internis cinereis, secundariorum cyanescentilus; subalaribus brunnescentibus, fusco variegatis; axillaribus subtus virescenti-cyaneis, supra brunnescentibus (!); paracercorum plumis longis, rectis, subrigidis, exterius brunneis, intus ad basin ultra- 
marinis, apicem versus violaceo-lilacinis, brevibus, plus minusve curvatis, ad basin cyaneis, medio ultramarinis, apicem versus cyaneis, paulum virescentibus; harum tectricibus brevioribus nigerrimis, longioribus curvatis, pulchre castaneis; rectricibus duabus intermediis longissimis, angustis, spatuliformibus, supra violascentibus, subtus nigris, spatulis macula cyanea ornatis; rostro flavido-albo; pedibus brunnescentibus (?); iride brunnea (Finsch et Meyer).

Long. tot. circa $0^{\mathrm{m}}, 270$; al. $0^{\mathrm{m}}, 160$; caud. $0^{\mathrm{m}}, 080$; rectr. interm. $0^{\mathrm{m}}, 440$; rostri $0^{\mathrm{m}}, 038$; tarsi $0^{\mathrm{m}}, 037$; parac. plum. $0^{\mathrm{m}}, 270$.

Foem. Capite, collo et summo dorso nitide obscure purpureo-nigris; colli plumis lateralibus et posticis longis; palpebris sicut in mari; summi dorsi plumis longis, velutinis, parte media obtecta et subtus virescenti-cyaneis; supracaudalibus et cauda cyanescenti-caeruleis; alis sicut in mari; gula virescenti nigra; gutture et pectore summo obscure castaneis, obsolete nigro transfasciolatis, plumis subtus plus minusve virescenti tinctis; abdomine summo virescenti-brunneo, medio et imo brunnescentibus, nigro transfasciolatis, medio plumarum apicibus cyaneis, omnibus subtus cyaneo-virescentibus; hypochondrii plumis longis, obscure brunneis, nigro transfasciolatis, subtus virescenti-cyaneis; tibiis nigris; crisso et subcaudalibus rufescentibus; cauda subtus virescenti-cyanea, transfasciolata; rostro flavido-albo; pedibus brumescentibus (?); iride brunnea (Finsch et Meyer).

Long. tot. circa $0^{\mathrm{m}}, 270 ;$ al. $0^{\mathrm{m}}, 153$; caud. $0^{\mathrm{m}}, 094 ;$ rostri culm. $0^{\mathrm{m}}, 032$; tarsi $0^{\text {nn }}, 048$.

Hab. in Papuasia - Nora Guinea orientali-meridionali (Hunstein, Forbes).

È questa una specie bellissima, il maschio della quale si distingue da tutte le Paradisee finora conosciute per la forma e pel colore ceruleo delle lunghe piume dei fianchi ; anche le ali e la coda, tanto del maschio, quanto della femmina, sono di colore ceruleo.

La Paradisornis rudolphi, cosi denominata in onore dell'infelice Arciduca Rodolfo d'Austria, cultore anch'esso della Ornitologia, fu scoperta dall'Hunstein nella Nuova Guinea orientale, ma la precisa località non è indicata; soltanto sappiamo che egli la trovò in una regione ostile, nella quale egli potera penetrare soltanto. inosservato dai nativi, e non senza pericolo della sua vita.

Nulla sappiamo ancora dei suoi costumi. Il Finsch ed il Meyer fanno notare come, sotto certe incidenze di luce, le piume di questo uccello perdano completamente il loro splendore ed appaiano senza lucentezza, bruniccie e nericcie.

\section{(718) Xanthomelus aureus (Linn.).}

Xanthomelus aureus, Eudes-Deslongch., Ann. Mus. d'Hist. Nat. Caen, I, p. 39 (1880). - Ramsay (nec Linn.?), Pr. Linn. Soc. N. S. W. X, p. 244 (1885). - Guillem., P. Z. S. 1885, p. 657. D’Hamonv., Bull. Soc. Zool. de Fr. 1886, pp. 508, 511.

Oriolus aureus, Musschenbr., Dagboek, pp. 209, 236 (1883). - Rosenb., Mith. Orn. Ver. Wien, 1885, p. 54.

Oriolus xanthogaster, Rosenb., ibid.

Il Ramsay attribuisce ad esemplari di questa specie le piume che si trovano su ornamenti del capo usati dagli indigeni della Nuova Guinea orientale-meridionale, ma non è improbabile invece che esse appartengano ad esemplari dell'affine Xanthomelus ardens, D'Alb. et Salvad. 
(719) Xanthomelus ardens, D'AlB. et SALVAD.

Oriolus ardens, Musschenbr., Dagboek, pp. 210, 237 (1883)。 - Rosenb., Mitth. Orn. Ver. Wien, 1885, p. 54 .

(720) Ghlamydodera cerviniventris, Gould.

Chlamydodera cerviniventris, Sharpe, Cat. B. VI, p. 393 (1881). - Musschenbr., Dagboek, p. 214 (1883). - Finsch, Võg. d. Südsee, p. 27 (Port Moresby) (1884). - Ramsay, Tab. List, p. 11 (1888).

Ptilonorhynchus cerviniventris, Hosenb., Mitth. Orn. Ver. Wien, 1885, p. 54.

(721) Amblyornis inornata (NCHLEG.).

Amblyornis inornata, Sharpe, Cat. B. VI, p. 394 (1881).

Ptilonorhynchus inornatus, Musschenbr., Dagboek, pp. 212, 242 (1883). - Rosenb., Mitth. Orn. Ver. Wien, 1885, p. 54.

\section{SP. 1151 (721 bis) Amblyornis subalaris, SHARPE.}

Amblyornis subalaris, Sharpe, Journ, Linn. Soc, Zool. XVII, p. 408 (1884). - Finsch u. Meyer, Zeitschr. f. ges. Orn. II, p. 390, tah. 22 (1885). - Id., Ibis, 1886, p. 257. - Sharpe, Nature, vol. 34. p. 340 (1886). - Id., Gould's B. New Guin. pt. XXII, pl. 9 1886). - D'Hamonv., Bull. Soc. Zool. de Fr. 1886, p. 511. - Ramsay, Pr. Linn. Soc. N. S. W. ser. 2a, vol. II, p. 250 (1887).

Mas. Supra olivaceo-brunneus, capitis crista copiosa, longissina, aurantiocomubra, antice fusco maculata; fronte nigricante; subtus brunneus, plumis in medio fulvo-olivaceis; lateribus obscurioribus; remigibus subtus fuscis, in pogonio interno olivaceo marginatis; subalaribus et axillaribus fulvo-aurantiacis; rostro nigricante; pedibus plumbeis.

Long. tot. $0^{\mathrm{m}}, 235$; al. $0^{\mathrm{m}}, 127$; caud. $0^{\mathrm{m}}, 097$; rostri $0^{\mathrm{m}}, 019$; tarsi $0^{\mathrm{m}}, 031$.

Foem. Mari simitis, sed crista aurantiaca destituta.

Long. 'tot. $0^{\mathrm{m}}, 215$; al. $0^{\mathrm{m}}, 116-0^{\mathrm{m}}, 122$; caud. $0^{\mathrm{m}}, 089-0^{\mathrm{m}}, 090$; rostri $0^{\mathrm{m}}, 019$; tarsi $0^{\mathrm{m}}, 030-0^{\mathrm{m}}, 031$.

Hab. in Papuasia - Nova Guinea meridionali-orientali, in Montibus Astrolabii (Goldie), Montibus Owen Stanley (Hunstein).

Questa specie si riconosce facilmente allo splendido ciuffo colore arancio del maschio ed alle piume delle parti inferiori ocracee nel mezzo, tanto nel maschio, quanto nella femmina; il becco è notevolmente più piccolo di quello dell' $A$. inornata.

Lo Sharpe descrisse da prima la femmina di questa specie; il maschio, scoperto dallo Hunstein, fu descritto dal Finsch e dal Meyer.

Lo Sharpe dice che il maschio nella stagione piovosa manca del ciuffo arancio e si può distinguere dalla femmina soltanto per avere le parti inferiori più ocracee. Egli suppone che anche il maschio dell' $A$. inornata possa avere un bel ciuffo, ma questa cosa è contraddetta ilalla numerosa serie d'individui tutti simili fra loro, raccolti sui monti Arfak.

Una cosa che non è stata finora notata è che il colore arancio del ciuffo del maschio dell' $A$. subalaris indica stretti rapporti delle specie del genere Amblyomis con quelle del genere Xanthomelus, e quindi viene confermata la opinione già da me emessa (Orn. Pap. e MLol. II, p. 663) che il Xanthomelus aureus sia affine alle specie 
del genere Chlamydodera, e che debba perciò essere annoverato nel gruppo dei Ptilonorinchini, anzichè delle Paradiseinae, e infine che molto probabilmente costruisca anch'esso un pergolato.

(722) Aeluroedus melanotis (G. R. GR.).

Aeluroedus melanotis, Salvad., Voy. Chall., Birds, p. 82 (1881). - Sharpe, Gat. B. VI, p. 383 (1881). - Neyer, Zeitschr. f. ges, Orn. I, p. 293 (1884).

Ptilonorhynchus melanotis, Musschenbr., Dagboek, pp. 212, 240 (1883). - Rosenb., Mitth. Orn. Ver. Wien, 1885 , p. 54 .

(723) Aeluroedus arfakianus, Meyer.

Aeluroedus arfakianus, Sharpe, Cat. B. VI, p. 384 (1881). - D'Hamonv., Bull. Soc. Zool. de Fr. 1886 , p. 511.

Ptilonorhynchus arfakianus, Nusschenbr., Dagboek, pp. 212, 241 (1883). - Rosenh., Mitth. Orn. Ver. Wien, 1885, p. 54.

\section{SP. 1152 ( $723^{\text {bis}) ~ A e l u r o e d u s ~ m e l a n o c e p h a l u s, ~ R A M S A T . ~}$}

Aeluroedus melanocephalus, Ramsay, Pr. Linn. Soc. N. S. W. VIII, p. 25 (1882). - Salvad., Ibis, 1884, p. 354. - Finsch u. Meyer, Zeitschr. f. ges. Orn. II, p. 394 (1885). - Id., Ibis, 1886, p. 258. - D'Hamonv., Bull. Soc. Zool. de Fr. 1886, p. 511. - Sharpe, Gould's B. New Guin. pt. XXIV, pl. 13 (1888).

Aeluroedus Ae. arfakiano, Meyer, similis, sed fascia nuchali nigra et maculis tectricum alarum mullis, jugulo et gutture variegatis, pectore et abdomine brunnescentibus diversus (Meyer).

Long. tot. $0^{\mathrm{m}}, 290$; al. $0^{\mathrm{m}}, 152$; caud. $0^{\mathrm{m}}, 120$; rostri $0^{\mathrm{m}}, 033$; tarsi $0^{\mathrm{m}}, 044$.

Hab. in Papuasia - Nova Guinea meridionali-orientali, Montibus Astrolabii (Funstein, Rolles).

Le contraddittorie ed insufficienti descrizioni di questa specie non mi lasciano afferrare $\mathrm{i}$ caratteri differenziali della medesima e quindi ho riferito la diagnosi data dal Meyer, sebbene la medesima sia in contraddizione colla figura publicata dallo Sharpe, nella quale appaiono evidenti le macchie chiare sulle cuopritrici delle ali, che il Meyer dice mancanti.

Il Meyer aggiunge che questa specie è intermedia allo Ae. arfakianus ed allo Ae. melanotis delle isole Aru, ma che somiglia più al primo; per la gola non nera si arvicina allo Ae.mclanotis, quantunque anche nell' Ae.melanocephalus la gola sia alquanto scura; per la testa scura somiglia allo Ae. arfalcianus, ma non ha la fascia cervicale nera nettamente definita; le cuopritrici verdi uniformi delle ali (che lo Sharpe rappresenta macchiate!) ricordano l'Ae. buccoides e l'Ae. stonei. L'Ae. melanocephalus sembra più scuro dell'Ae. arfakianus nella parte inferiore della coda, che arrebbe una fascia terminale più stretta. Le piume della gola nello Ae. melanocephalus sono nere alla base ed all'apice ed hanno una larga fascia intermedia bianca, cosicchè vi predomina il bianco, laddove nello $A e$. arfakianus predomina il nero; le parti inferiori sono tinte di olivaceo-bruno e sono più oscure di quelle dello Ae. arfakianus e dello Ae. melanotis.

Inoltre $10^{\circ}$ Sharpe dice che l'Ae. melanocephalus differisce dallo Ae. arfakianus per le redini e pel mento neri e pel colorito uniforme del petto e dell'addome, i quali caratteri non appaiono nella figura. 


\section{(724) Aeluroedus buccoides (TEMr.).}

Aeluroedus buccoides, Sharpe, Cat. B. VI, p. 386 (1881). - Jehrk., Journ. f. Orn. 1885, p. 34 (Waigeu). - Guillem., P. Z. S. 1885, p. 657 (Dorei, N. New Guin. long. $139^{\circ}$ E. (Bruijn), Jubi, Batanta, Salwatti).

Ptilonorhynchus buccoides, Musschenbr, Dagboek, pp. 211, 240 (1883). - Rosen b., Mitth. Orn. Ver. Wien, 1885̃, p. 54 .

Jobi è una nuova località per questa specie.

\section{(725) Aeluroedus stonei, SHARpE.}

Aeluroedes stonii, Sharpe, Cat. B. VI, p. 387 (1881). - Finsch et Meyer, Zeitschr. f. ges. Orn. II, p. 391 (1885). - Iil., Ibis, 1886, p. 258. - D'Ilamonv., Bull. Soc. Zool. de Fr. 1886, p. 511.

Ptilonorhynchus Stonei, Musschenbr., Diıboek, pp. 212, 241 (1883). - Rosenb., Mitth. Orn. Ver. Wien, 1885 , p. 54.

Il Finsch ed il Meyer menzionano un esemplare, raccolto dallo Hunstein, avente la testa verdognola con strie giallognole sul mezzo e la fronte giallognola; secondo essi questi caratteri sarebbero i resti dell'abito imperfetto. 



\section{AGGIUNTE}

ALLA

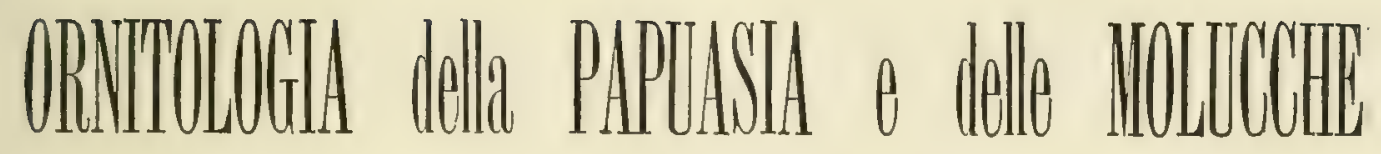

PER

TOMMASO SALVADORI

PARTE TERZA

COLUMBAE - GALLINAE - GRALLATORES

ANSERES - STRDTHIONES

T O R I N 0

C A R L C L A U S E N

Libraio della R. Accademia delle Scienze 
Estratto dalle Memorie della $R$. Accademia delle Scienze di Torino SERIE II, TOM. XLII

Torino, Stamperia Reale-Paravia.

672 (C) $30-V I 1-91$ 


\title{
AGGIUNTE \\ ALLA \\ Ornitologia della Papuasia e delle Molucche PER
}

\author{
TOMMASO SALVADORI
}

\section{PARTE TERZA}

\author{
COLUMBAE - GALLINAE - GRALLATORES \\ ANSERES - STRUTHIONES
}

\section{APPENDICE}

Appr. nell'adunanza del 10 Naggio 1891

\section{PREFAZIONE}

In questa terza ed ultima parte delle Aggiunte alla Ornitologia della Papuasia e delle Molucche io mi propongo di condurre a termine il mio lavoro, passando in rassegna tutte quelle aggiunte che si sono fatte agli ordini Columbae, Gallinae, Grallatores, Anseres e Struthiones, dopo la pubblicazione del terzo volume della Ornitologia, avvenuta nel 1882; inoltre, siccome, durante il tempo corso dalla pubblicazione della prima parte delle Aggiunte fino ad ora, si sono scoperte molte cose nuove relative anche agli Ordini Accipitres, Psittaci, Picariae e Passeres, dei quali ho discorso nelle prime due parti, perciò stimo opportuno di aggiungere un'Appendice, nella quale siano annoverate tutte quelle novità, e per tal modo il mio lavoro possa dirsi compiuto alla data della sua pubblicazione.

Alle specie degli ordini Calumbae, Gallinae, Grallatores, Anseres e Struthiones, annoverate nel volume III della Omitoiogia, sono ora da aggiungerne 18 ; altre 28 specie spettano agli ordini precedenti, cosicchè avrò da aggiungere e descrivere 46 specie, delle quali dò qui l'elenco :

1. Ptilopus flavovirescens, Meyer.

2. " quadrigeminus, Meyer.

3. " plumbeicollis, Meyer.

4. 》 lettiensis, Schleg.

5. Carpophaga rubiensis, Meyer.
6. IIyristicivora subflavesecns (Finsch).

7. Macropygia timorlaoensis, Meyer.

8. » cinereiceps, Tristr.

9. Eutrygon leucopareia, Meyer.

10. Otidiphaps insularis, $\mathrm{S}$. et $\mathrm{G}$. 
11. Megapodius tenimberensis, Sclat.

12. Rallina woodfordi, Grant.

13. Rallicula forbesi, Sharpe.

14. Oedicnemus grallarius (Lath.).

- Heteractitis brevipes (Vieill.).

15. Numenius lineatus, Cuv.

16. Ardeiralla woodfordi, Grant.

17. Nycticorax mandibularis, Grant.

18. Platalea intermedia, Grant.

\section{APPENDICE.}

19. Ninox goodenoviensis, De Vis.

20. 》 rosseliana, Tristr.

21. Geoffroyus sudestiensis, De Vis.

22. Cyclopsittacus nigrifons, Rchnw.

23. Merops salvadorii, Meyer.

24. Tanysiptera rosseliana, Tristr.

25. Sauromarptis lubaryi, Meyer.

26. Eurystomus sa7omonensis, Sharpe.

27. Aegotheles Toriae, Salvad.
28. Monarcha florenciae (Sharpe).

29. 》 leucophthalmus (Ramsay).

30. Arses orientatis, Salvad.

31. Rhipidura auricularis, De Vis.

32. Poecilodryas sigillata, De Vis.

33. Todopsis kovaldi, De Vis.

34. Graucalus longicauda, De Vis.

35. Colluricincla discolor, De Vis.

36. Myzomela pulchella, Salvad. nor. sp.

37. Metirrhophetes belfordi, De Vis.

38. Ptitotis aruensis, Sharpe.

39. 》 gracilis, Gould.

40. Zosterops pallidipes, De Vis.

41. Pitta Toriae, Salvad.

42. Merula papuensis, De Vis.

43. Munia nisoria (Temm.).

- Erythrura forbesi, Sharpe.

44. Paryphephorus duivenbodei (Meyer).

45. Cnemophitus macgregorii, De Vis.

46. Amblyornis musgraviana, Goodw.

Di queste 46 specie, una, il Numenius lineatus, Cuv., fu da me ommessa per inavvertenza nella Ornitologia; tre, il Ptilopus lettiensis, Schleg., I'Dedicnemus grallarius (Lath.) e la Munia nisoria (Temm.), sono state trovate recentemente nelle isole del gruppo di Timor-laut; una quinta, la Plilotis gracilis, Gould, viene ora considerata da me come distinta dalla P. analoga, colla quale l'aveva prima unita; una sesta, la Myzomela pulchella, viene ora descritta per la prima volta sopra un esemplare del Museo Britannico, e finalmente le altre 40 sono state denominate e descritte negli ultimi tempi, e tra queste meritano speciale menzione due della famiglia dei Paradiseidi, ciascuna tipo di un nuovo genere, e queste sono il Pamphephoms duivenbodei (Meyer) ed il Cnemophilus macgregorii, De Vis, scoperto sui Monti Oren-Stanley ad 11,000 piedi di altezza, durante. la recente spedizione del MacGregor.

Nell'elenco delle specie aggiunte ho lasciato senza numero la Heleractitis brevipes e la E'ythrura forbesi, le quali prendono rispettivamente il posto del Totanus incanus e della Evythrura tricolor, da me precedentemente annoverate.

Colle aggiunte sopra indicate il numero delle specie di uccelli della Papuasia e delle Molucche, ossia della regione da me sturiata, è di 1198 , quasi un decimo del numero delle specie conosciute.

Torino, Museo Zoologico, maggio 1891. 


\section{BIBLIOGRAFIA}

\section{AGGIUTTE Al LAVORI RELATIVI ALLA AVIFAUIA DELLA SOTTO-REGIONE PAPCANA

\author{
pubblicati dal 1881 al 1891.
}

(1881) FINSCH, 0., Ornithological letters from the Pacific. VIII, New Britain (Ibis, 1881, p. $532510 ;.$

(1884) OUSTALET, M. E., Notices sur quelques espèces notivelles ou peu connues de la Collectiun Ornitbologique du Muséum, II. Description de trois espèces d'oiseaux originaires de la Nouvelle Guinée, etc. (Nouv. Arch. du Mus. d'Hist. Na!., deuxième série, Tome VIII, p. 300-303, pl. XII).

(1884) SHARPE, R. B., Report on the Zoological Collections made in the Indo-Pacific Ocean during the Voyage of H. H. S. « Alert» 1881-82, London, 1884.

(In questo lavoro è descritta la Plilotis aruensis, Sharpe).

(1886) D'HAMONVILLE, Baron, Nouveautés Ornithologiques - Puradisiers. Revue de quelques espèces récemment découvertes, description d'un état de plumage anormal et tableau des espèses (Bull. de la Soc. Zool, de France, 1886, pp. 503-511).

(1887) BARTLETT, A. D., Remarks upon the Moulting of the Great Bird of Paradise (P. Z. S. 1887, p. 392).

(1887) NORTH, A. J., Contribution to the Uology of the Austro-malayan and Pacific Regions (Pr. Linn. Soc. N. S. W. (2) II, p. 441-446, 1887).

(Sono descritte le uova di molte specie).

(1887) OUSTALET, E., Description du mâle adulte du Drepanornis Bruijnii, Oust. (Le Naluraliste, 1887 , pp. 180-182).

Questu importante articolo è sfuggito agli editori delle hiviste scientifiche e non si trova menzionato nell' Ibis, nel Journal für Ornithologie e neppure nel Zoological Record pel 1887).

(1888) FINSCH, Dr O., Samoafahrten, Reisen in Kaiser Wilbelms-Land und English Neu-Guinea in den Jahren 1884 u. 1885 an Bord des deutschen Dampfers \& Samoa ». Leipzig, 1888.

(Vi è denominata e brevemente descritta una Cacalua trobriandi, che lo stesso Finsch (in litt.) dice non diversa dalla $C$. triton). 
(1898) WOODFORD, C. M., General Remarks on the Zoology of the Solomon Islands, and Notes on Brenchley's Megapode (P. Z. S. 1888, pp. 248-250;.

(1889) GABAiIS, J., Paradisea Augustae Vicloriae (Journ. C. Orn. 1889, Tav. II).

(1889) OGILVIE-GRANT, W. R., On the Genus Turnix (Ibis, 1889, pp. 446-475).

1889) OGILVIE-GRAIT, IV. R., Third Cuntribution to the List of Birds collected by Mr. C. M. Woodford in the Solomon Archipelago (Ann. and Mag. N. H. (6) IV, p. 320, 1889).

(È descritta la Rallina woodfurdi).

(1889) ThistraM, H. B., Catalogue of a Collection of Birds. Durham, 1889.

(1889) TRISTliAM, H. B., On a small Collection of Birds from the Louisiade and d'Entrecasteaux Islands (Ibis, 1889, pp. 553-558;:

(Sono descritte le seguenti specie nuove : Manucodia thomsoni, - Craclicus Iouisia. densis, - Dicaeum nilidum, - Cinnyris christianae, - Chibia propinqua, - Tanysiplem rosseliana, - Ninox rosseliana, - Macropygia cinereiceps!.

(1889) Parliamentary Report on the British Nuseum (Ibis, 1889, pp. 581-583).

(1889) GOODWIT, A. P., Exhibition of, and remarks upon, some Paradise-birds, procured during Sir William Macgregor's recent expedition to Mount Owen-Stanley, New Guinea (P. Z. S. 1889, p. 451).

(E) descritta l'Amblyornis musgravii!.

(1889) MEYER, A. B., Beschreibung der bisher unbekannten Weibchen von Astrarchia Stephaniae und Epimachus macleayanae (Journ. f. Orn. 1889, p. 321-326).

(1890) G0ODWIN, A. P., Notes on the Paradise-birds of British Nerv Guinea (Ibis, 1890, pp. 150-156). (È descritto incompiutamente il Xanthomelus macgregorii).

(1890) SHARPE, R. B., Descriptions of three new species of Flycatchers (Ibis, 1890, p. 205).

(1890) RAMSAY, E. P., Report on a zoological Collection from the Solomon Islands. Part I (Records of the Australian Misseum, I, pp. 3-5, March 1890).

(È descritta la Pomarea loucophthalmus).

(1890) SHARPE, R. B.; Notes on Specimens in the Hume Collection. ‥ 6. On the Coraciidae of the Indian Region, with Descriptions of some new Species (P. Z. S. 1890, pp. 546-552).

(Ẻ descritto l'Euryslomus solomonensis,.

(1890) SHARPE, R. B., Catalogue of the Birds in the British Museum, XIII, 1890.

(La Erylhrura forbesi viene separata dall'E. tricolor e descritta come nuova).

(1890) WARDLAW RAMSAY, R. G., On the Columbine genus Macropyjia and its Allies (Ibis, 1890, рp. 214-246).

(1890) WARDLAW IRAMSAY, R. G., On a nerv Genus of the Order Columbae (Ibis, 1890, pp. 246-247)

(1890) DE VIS, C. W., Report on Birds from British New Guinea (Annual Report on British New Guine from $4^{\text {th }}$ September 1888 to 30th June 1889. Appendix G. pp. 57.62, Brisbane 1890. - Colonial Papers, no. 103, Brilish New Guinea, Appendix G. pp. 105-116, London 1890. - Ibis, 1891, пр. 25-41;

(In questo lavoro, la cui pubblicazione originale è sfuggita all'Editore dell'Ihis, che to ha riprodotto dalle Colonial Papers, sono descritte tredici nuove specie: Ninox goodenoviensis, - Geoffroyus sudestiensis, - Rhipidura auricularis, - Poecilodryas (?) sigillula, - Todopsis kovaldi, - Graucalus longicaula, - Strepera rosa-alba (= Gracticus louisindensis, Tristr.), - Colluricincla discolor (=? Pachycephala forlis, Gadow). - Melirrhoph tes belfordi, - Zosierops pallidipes, - Merula papuensis, - Amblyornis macgregoriae (= Amblyornis musgravi, Goodw.) - Cnemophilus macgregorii). 
(1890) SALVADURI, T., Viaggio di Lamberto Loria nella Papuasia orientale, II. Collezioni Ornitologiche. Nota seconda. Uccelli della Nuova Guinea meridionale-orientale (Ann. Ius. Civ. Gen. (2) IX, pp. 554-592).

(Vengono descritte tre nuove specie: Aegotheles Loriae, Arses orientalis, Pitla Loriae).

(1890) Heine, F. et REICHENoW, A., Nomenclator Musei Heineani Ornithologici, Berlin, 1882-1890.

(1890) MEYER, A. B., Notes on Birds from the Papuan Region with Descriptions of some new Species (Ibis, 1890, p. 412-424).

(Sono descritte le seguenti specie : Sauromarplis kubaryi, Craspedophora duyvenbodei, Plilopus quadrigeminus e Plilopus plumbeicollis).

(1891) MEYER, A. B., Letter on a new Species of Bee-eater from New Britain (Ibis, 1891, p. 293). (E) descritto il Merops Salvadoriı).

(1891) SALVADORI T., Viaggio di Lamberto Loria nella Papuasia orientale. III, Collezioni Ornitologiche. Nota terza. Uccelli della Nuova Guinea meridionale-orientale e delle isole di. Entrecasteaux (Ann. Ifus. Civ. Gen. (2) X, pp.797-834).

(1891) REICHENOW, Dr. A., Cyclopsillacus nigrifrons, n. sp. (Allg. Deutsche Ornith. Gesell. zu Berlin. Bericht III, p. 6, 20 März 1891. - Journ. f. Orn. 1891, p. 217). 


\section{ORDo COLUMBAE}

Gen. PTIlopus, Sw.

Terenotreron, Heine, Nomencl. Mus. Hein. Orn. p. 280 (1890) i=Thouarsitreron, Bp.).

(728) Ptilopus xanthogaster (WAGL.).

Terenotreron zanthogaster, Heine et Rchnw., Nomencl. Mus. Hein. Orn. p. 280 (1890)(Aru !).

Sp. 1158 (728 ${ }^{\text {is }}$ ) Ptilopus flavovirescens, MrXer.

Ptilopus xanthogaster, Meyer (nec Wagl. \&), Verh. z.-b. Ges. Wien, 1881, p. 769 (Lettie) (8). Sclat. (nec Wagl.), P. Z. S. 1883, pp. 51, 195, 200. - Forbes, P. Z. S. 1884, pp. 430, 433, n. 47. - Büttik., Not. Leyd. Mus. VIII, p. 66 (1886) - Tristr., Cat. Coll. B. p. 45 (1884) (Tenimber Island).

Ptilopus diadematus, part., Schleg., Mus. P. B. Columbae, p. 12 (individus de l' fle de Lettie) (1873).

Ptilopus flavovirescens, Meyer, Sitzb. u. Abb. der Gesellsch. Isis, Abh. I, p. 50 (1884) (Timorlaut, Tenimber) - Salvad., Ibis, 1884, p. 355. - Id., P. Z. S. 1884, p. 580.

Ptilopus Pt. xanthogastri simitis, sed capite et collo flavovirescente tinctis.

Hab. in Papuasia - Ins. Timorlaut et Tenimber (Forbes, Ricdel); Letti (Hoedt, Riedel).

Io ho esaminato quattro esemplari di questa forma; essi differiscono indubitatamente da quelli delle Isole Kei, di Banda e di Khoor per avere la testa ed il collo di colore più decisamente verdognolo, lo scudo pettorale alquanto più cupo e per altri rispetti; tuttavia non oso pronunciarmi nel dare a quelle differenze valore specifico, tanto più anche gli esemplari di Lettie, di Khoor e di Banda differiscono alquanto fra loro.

(1021) Ptilopus richardsi, Ramisax.

Ptilopus richardsi, Ramsay, Pr. Linn. Soc. N. S. W. VII, p. 39 (1882) (Isole Salomone). - Sclat., P. Z. S. 1883, p. 348. - Sharpe in Gould's B. of New Guin. pt. XVIII, pl. 3 (1884).

Ptilopus rhodosticus, Ramsay, I. c. p. 43 (1882). - Tristr., Cat. Coll. B. p. 45 (1889).

\section{(729) Ptilopus superbus (Tемм.).}

Ptilopus superbus, Ramsay, Pr. Linn. Soc. N. S. W. VII, p. 38 (1882) (Isole Salomone). Tristr., Ibis, 1882, p. 144. - Sharpe, Journ. Linn. Soc. Zool. XVl, p. 445 (China Straits) (1882). - Blas. u. Nehrk., Verh. z.-b. Ges. Wien, 1882, p. 428 (Amboina). - Ramsay, I.c. VIII, p. 28 (uova) (1883). - Finsch, Vög. d. Südsee, pp. 18, 28 (Nova Britannia, Laloki, Somerset)(1884). Pleske, Bull. Acad. Petersb. XXIX, p. 534 (Ternate) (1884). - Nehrk., Journ. f. Orn. 1885, p. 34 (Waigeu). - Guillem., P. Z. S. 1885, p. 574 (Ternate), 657 (Mysol). - Grant, P. Z. S. 1888, p. 199. - Tristr., Cat. Coll. B. p. 45 (1889). - Salvad., Ann. Mus. Giv. Gen. (2) IX, p. 586 (1890). - De Vis, Ann. Rep. Brit. New Guin. p. 62 (1890\%. - Id., Colon. Papers, no. 103, p. 116 (1890). Id., Ibis, 1891, p. 40. - Salvad., Ann. Mus. Civ. Gen. (2) X, pp. 823, 833 (1891) (Isola Goodenough). Ptilonopus superbus, Ramsay, Tab. List, pp. 17, 30 (1888).

Hab. Insula Sudest dicta. 
(730) Ptilopus pulchellus (TEMm.).

Ptilopus pulchellus, Sharpe, Journ. Linn. Soc., Zool. XVI, p. 445 (Taburi) (1882). - Ramsay, Pr. Linn. Soc. N.. S. W. VIII, p. 28 (1883) (uovo). - Nehrk, Journ. f. Orn. 1885, p. 34 (Waigeu). Guillem., P. Z. S. 1885, p. 657. - Finsch u. Meyer, Zeitschr, f. ges. Orn. 1886, p. 26 (Monti Hufeisen). - Tristr., Cat. Coll. B. p. 44 (1889). - Heine et Ilchnw., Nomencl. Mus. Hein. Orn. p. 280 (1890) (Ceram !). - Salvad, Ann. Mus. Civ. Gen. (2) X, p. 823 (1891).

(781) Ptilopus coronulatus, G. R. GR.

Ptilopus coronulatus, Tristr, Cat. Coll. R. p. 44 (1889), - Salvad, Ann. Mus, Civ. Gen. (2) IX, p. 586 (1890) (Rigo, Nuova Guinea meridionale orientale); X, p. 823 (1891) (Igibirei).

\section{(782) Ptilopus trigeminus, Salvad.}

? Ptilopus pulchelloides, Bernst., Dagboek, pp. 124, 140 (Sorong) (1883) (descriptio nulla). ? Ptilopus flavigaster, Bernst. (nec Sw。) op. cil. p. 154 (Sailolo) (1883).

(733) Ptilopus geminus, SaLvad.

Ptilopus geminus, Meyer, Sitzb. u. Abh. Gesellsch. Isis, 1884, Abh. I, p. 50 (uova). - Guillem., P. Z. S. 1885 , p. 658 (Jobi). - Meyer, Zeitschr. f. ges. Orn.1886, p. 36 (Kafu).

Sp. 1154 (798bis) Ptilopus quadrigeminus, Mryer.

Ptilopus quadrigeminus, Meyer, lbis, 1890, p. 421 (Constantine Harbour).

*Pt. gemino, Salvad. similis, sed capitis lateribus et gula cinereis vix virescentibus; pileo lilacino paullo laetiore, postice linea purpurea nulla; area abdominis crocea paullo pallidiore diversus.»

« Long. al. $0^{\mathrm{m}}, 115$; caud. $0^{\mathrm{m}}, 066$; rostri $0^{\mathrm{m}}, 012$; tarsi $0^{\mathrm{m}}, 015 »$ (Meyer).

Hab. Sinu Constantini, Nova Guinea septentrionali (Kubary).

(734) Ptilopus monachus (REINw.).

Ptilopus monachus, Pleske, Bull. Acad. Petersb. XXIX, p. 531 (Ternate) (1884)。 - Guillem., P. Z. S. 1885, p. 575 (Halmahera). - Tristr., Cat. Coll. B. p. 44 (1889).

(735) Ptilopus humeralis, WALL.

Ptilopus humeralis, Guillem., P. Z. S. 1885, p. 658 (Waigiou). - Tristr., Cat. Coll. B. p. 44 (1889),

Waigiou è una località nuova per questa specie.

(787) Ptilopus iozonus, G. R. GR.

Ptilopus iozonus, Tristr., Cat. Coll. B. p. 44 (1889). - Salvad., Ann. Mus. Civ. Gen. (2) IX, p. 587 (1890) (Rigo); X, p. 823 (1891) (Igibirei).

(738) Ptilopus insolitus, BCHLEG.

Aedirhinus (sic) insolitus, Haswell, Pr. Linn. Soc. N. S. W. VIJ, p. 115 (1882) (anatomia). Oedirhinus insolitus, Finsch, Vög. d. Südsee, p. 18 (Nova Britannia) (1884).

Ptilopus insolitus, Tristr., Cat. Coll. B. p. 44 (1889).

(789) Ptilopus aurantiifrons, G. R. GR.

Ptilopus aurantiifrons, Finsch u. Meyer, Zeitschr. f. ges. Orn. 1886, p. 26 (Aroani, pressoMilne Bai). - Tristr., Cat. Coll. Birds, p. 43 (1889). 
SdBgen. SYLPHITRERON, VERr.

Poecilotreron, Heine, Nomencl. Mus. Hein. Orn. p. 281 (1890). (= Sylphidoenas, Bp.).

(740) Ptilopus wallacei, G. R. Gr.

Ptilopus wallacei, Sharpe in Gould's B. New Guin. pt. XV, pl. 9 (1883). - Sclater, P. Z. S. 1883 , p. 51, 195, 200. - Meyer, Sitzb. u. Abh. Gesellsch. Isis, 1884, Abh. I, p. 50. - Forbes, P. Z. S. 1884, p. 433. - Guillem., P. Z. S. 1885, p. 658 (Aru). - Büttik., Not. Leyd. Mlus. VIII, p. 66 (1886). - Tristr., Cat. Coll. B. p. 45 (1889) (Larat).

Poecilotreron wallacei, Heine et Rchnw., Nomencl. Mus. Hein. Orn. p. 281 (1890).

Questa specie è stata trovata anche in Timorlaut (Forbes) ed in Babbar (Riedel).

(741) Ptilopus ornatus, Rosens.

Ptilopus ornatus, Guillem., P. Z. S. 1885 , p. 658.

(742) Ptilopus gestroi, SALVAD. $Q$ D'ALB.

Ptilopus gestroi, Salvad., Ann. Mus. Civ. Gen. (2) X, p. 823 (1891) (Bujakori).

(743) Ptilopus perlatus (Tемн.).

Ptilopus perlatus, Nebrk., Journ, f. Orn. 1885, p. 34 (Waigeu). - Tristr., Cat. Coll. B. p. 44 (partim) (1889).

(744) Ptilopus zonurus, SALVAD.

Ptilopus perlatus, Tristro, Cat. Coll. B. p. 44 (part.) (1889) (Austrolabe Mountains).

Ptilopus zonurus, Salvad., Ann. Mus. Civ. Gen. (2) IX, p. 587 (1890) (Rigo); X, p. 824 (1891) (BaraBara, Igibirei, Bujakori).

\section{Sp. 1155 (744 $4^{\text {bis }}$ Ptilopus plumbeicollis, METer.}

Ptilopus plumbeicollis, Meyer, Ibis, 1890, p. 422 (Constantine Harbour).

«Similis Pt. perlato et Pt. zonuro, sed capite cinereo et collo postico plumbeo diversus.

«Long. al $0^{\mathrm{m}}, 155$; caud. $0^{\mathrm{m}}, 076$; rostri $0^{\mathrm{m}}, 021$; tarsi $0^{\mathrm{m}}, 021$. » (Meyer).

(745) Ptilopus strophium, Gould.

Ptilopus rivolii, part., Ramsay, Pr. Linn. Soc. N. S. W. III, p. $290(1878)$; IV, p. 100, no. 156 (1879). - Salvad., Ibis, 1880 , p. 325.

Ptilopus strophium, Meyer, Sitzb. u. Abh. der Gesellsch. Isis, 1884, p. 51. - Tristr., Ibis, 1889, p. 558.

Ptilopus rivolii, De Vis (nec Prev.), Ann. Rep. Brit. New Guin. p. 62 (1890). - Id., Colon. Papers, no. 103, p. 116 (1890). - Id., Ibis, 1891, p. 40.

Hab. in ins. East et Rossel dictis (Thomson); ins. St. Aignan (fide De Vis).

Il Meyer fece notare che un esemplare di Port Moresby presentava la fascia pettorale bianca tinta di giallo, e percio espresse il dubbio che esso non potesse essere riferito al $P$. strophium delle Isole Luisiadi, che venne descritto senza quella tinta gialla. Io ho potuto constatare su esemplari del Museo Britannico che mentre la tinta gialla è scomparsa nell'esemplare tipico, scolorato dalla lunga esposizione, essa si trova negli esemplari freschi delle Luisiadi, i quali sono simili in tutto a quelli di Port Moresby. 
(748) Ptilopus prasinorrhous, G. R. GR.

Ptilopus prasinorrhous, Blas, u. Nehrk., Verh。 zo-b. Ges. Wien. 1882, p. 428 (Amboina). Guillem., P. Z. S. 1885, p. 575 (Weeda IsI.), 658 (Traitors Islands, N. of Jobi, Bruijn). - Tristr., Cat. Goll. B. p. 44 (1889).

(749) Ptilopus bellus, Sclat.

Ptilopus bellus, Ramsny, Pr. Linn. Soc. N. S. W. VIII, p. 27 (1883) (nido ed uova). - Guillem., P. Z. S. 1885, p. 658 (Andai, Arfak). - Tristr., Gat. Coll. B. p. 43 (1889). - De Vis, Ann. Rep. Brit. New Guin. p. 62 (1890) (S. S. New Guinea passim to 4000 feet). - Id., Colon. Papers, no. 103, p. 116 (1890). - Id., Ibis, 1891, p. 40. - Salvad., Ann. Mus. (Giv. Gen. 2) X, p. 824 (1891) (Bujakori).

Ptilopus patruelis. Meyer, Zeitschr. f. ges. Orn.1886, p. 27, taf. VI (Nuova Guinea meridionaleorientale)

Ho esaminato due esemplari maschi della Nuova Guinea meridionale-orientale, uno raccolto sui monti dell'Astrolabio dal Goldie e l'altro sui Monti Owen-Stanley e non sono riuscito a trovare le differenze menzionate dal Meyer, siccome caratteristiche degli esemplari meridionali-orientali; anche una femmina di Bujakori non differisce dalle femmine di Hatam.

(750) Ptilopus speciosus, Rosens.

Ptilopus speciosus, Guillem., P. Z. S. 1885, p. 658 (Traitors Islands, N. of Jobi, Bruijn). - Tristr., Gat. Coll. B. p. 45 (1889).

(751) Ptilopus johannis, Sclat.

Ptilopus johannis, Sclat., P. Z. S. 1883, p. 348.

(752) Ptilopus salomonensis, G. R. GR.

Ptilopus johannis, Ramsay (nec Sclat.), Pr. Linn. Soc. N. S. W. VI, p. 724 (1881); VII, p. 39 (1882) (Isole Salomone).

Ptilopus salomonensis, Sclat., P. Z. S. 1883, p. 348. - Sharpe in Gould's B. New Guin. pt. XIX, pl. 8 (1885). - Grant, P. Z. S. 1888, p. 199.

Ptilopus ceraseipectus, Tristr., Cat, Coll, B. p. 43 (1889).

(754) Ptilopus nanus (Темм.).

Ptilopus nanus, Nehrk, Journ. f. Orn. 1885, p. 34 (Waigeu).

\section{(755) Ptilopus ionogaster (RerNw.).}

Ptilopus ionogaster, Pleske, Bull. Acad. Petersb. XXIX, p. 534 (Ternate) (1884). - Guillem., P. Z. S. 1885 , p. 575 (Halmahera).

Ptilopus iogaster, Tristr., Cat. Coll. B. p. 44 (1889).

(756) Ptilopus eugeniae (Goold).

Ptilopus Eugeniae, Ramsay, Pr. Linn. Soc. N. S. W. VII, p. 38 (1882) (Isole Salomone). - Sclat., P. Z. S. 1883, p. 348. - Tristr., Cat. Coll. B. p. 44 (1889).

\section{Ptilopus sp.}

Ptilopus eugeniae, De Vis (nec Gould.), Ann. Rep. Brit, New Guin. p. 62 (1890) (Ferguson Island). - Id., Colon. Papers, n. 103, p. 116 (1890). - Id., 1bis, 1891, p. 40.

Hab. Insula Ferguson (De Vis). 
Io credo che il $\boldsymbol{P}$ t. eugenide sia confinato nelle Isole Salomone, e che gli esemplari dell'Isola Ferguson dal De Vis attribuiti a detta specie debbano invece spettare ad una diversa.

\section{(757) Ptilopus viridis (LinN.).}

Ptilopus viridis, Blas, u. Nehrk., Verh. z..-b. Ges. Wien, 1882, p. 428 (Amboina). - Tristr., Cat. Coll. B. p. 45 (1889).

\section{(1022) Ptilopus lewisi, Ramsay.}

Ptilopus lewisi, Ramsay, Pr. Linn. Soc. N. S. W. VII, p. 38 (1882) (Isole Salomone). - Sharpe in Gould's B. of New Guin. pt. XVIl, pl. 3 (1884). - Grant, P. Z. S. 1887, p. 332; 1888, p. 199. Tristr., Lat. Coll. B. p. 269 (1889).

È questa una specie perfettamente distinta; essa, più che a qualunque altra specie, somiglia al $P$. musschenbroecki, avendo come questo la parte anteriore della testa di color grigio verdognolo e la grande area porporina sulla parte anteriore del collo e del petto, ma si distingue facilmente per avere quell'area circondata di porporino-rioletto più cupo.

(759) Ptilopus pectoralis (WAGr.).

Ptilopus pectoralis, Nehrk., Journ. f. Orn. 1885, p. 34 (Waigeu). - Guillem., P. Z. S. 1885, p. 659 (Mysol, Waigiou).

\section{SUbgends LEUCOTRERON, Br.}

Leucotreron, Bp., Compt. Rend. XXXIX, p. 876 (1854) . . . . Columba cincla, Temm.

Sp. 1156 (759 ${ }^{\text {bis }}$ ) Ptilopus lettiensis, Schleg.

Ptilopus cinctus lettiensis, Schleg., Ned. Tijuschr. Dierk. IV, p. 20 (1873). - Id., Mus. P. B. Columbae, p. 35 (1873) (Lettie).

Ptilopus lettiensis, Elliot, P. Z. S. 1878, p. 572. - Meyer Vehr. z. - b. Gesellsch. Wien, 1881, p. 770 (Luang). - Id., Zeitschr. f. ges. Orn. 1884, p. 213 (Timorlaut).

Capite, collo et gutture albis ; dorso nigro-ardesiaco, uropygio griseo-virescente; fascia pectorali transversa coeruleo-nigra; abdomine flavo-olivaceo; subcaudalibus albis, flavo marginatis, in medio fusco-viridibus ; alis et cauda nigroardesiacis, subtus griseis; caudae parte apicali albo-citrina.

Juv. Dorso et uropygio magis virescentibus; tectricibus alarum, praesertim majoribus et mediis aeneo-virescentibus et flavido marginatis; remigibus secundariis aeneo-virescentibus et pallide limbatis.

Long. tot. circa $0^{\mathrm{m}}, 298$; alt. $0^{\mathrm{m}}, 158$; caud. $0^{\mathrm{m}}, 116$; rostri culm. $0^{\mathrm{m}}, 015$.

Hab. in Papuasia - Ins. Timorlaut (Riedel), Luang (Riedel), Lettie (Hoedt).

Questa specie somiglia molto al Pt. cinctus (Temm.) di Timor ed al Pt. albocinctus, Wall. di Flores; si distingue facilmente da ambedue pel colore bianco-citrino della parte apicale (quasi la meta) della coda; dal $\boldsymbol{P}$. albocinctus differisce anche pel colore del collo che in questa specie è grigio-azzurrognolo.

Io arrei dovuto forse annoverare questa specie nel terzo volume della mia Or[54] 
nitologia, giacchè, secondo lo Schlegel, l'isola di Lettie, nella quale questa specie era gia stata trovata, appartiene al gruppo di Timor-laut, la quale cosa verrebbe confermata dal fatto che in Lettie non si trova il $P$. cinctus proprio di Timor, ma sibbene la specie che lo rappresenta in Timor-laut (1).

(760) Megaloprepia formosa G. R. GR.

Megaloprepia formosa, Pleske, Bull. Acad. Petersb. XXIX, p. 534 (Ternate) (1884).

\section{(761) Megaloprepia puella (Less.).}

? Carpophaga magnifica, Bernst. (nec Temm.), Dagboek, p. 161 (Batanta) ('883).

Megaloprepia puella, Nehrk., Journ. f. Orn. 1885, p. 34 (Waigev, novo). - Guillem., P. Z. S. 1885, p. 659 (Waigiou), Batanta, Mysol, Salwatti). - Tristr., Cat. Coll. B. p. 43 (1889) (Mysol). Heine et Kchnw., Nomencl. Mus. Hein. Orn. p. 282 (1890) (Ceram I).

\section{(762) Megaloprepia poliura, Salvad.}

Carpophaga poliura, Ramsay, Pr. Linn. Soc. N. S. W. VIII, p. 28 (1883) (uovo).

Megaloprepia assimilis, Finsch (nec Gould), Vög. đ. Südsee, p. 28 (Fiu ni Goldie e Laloki) (1884).

Megaloprepia poliura, Meyer, Zeitschr. f. ges. Orn. 1886, p. 37 (Kafu). - Ramsay, Tab. List, nota 530a (1888). - Tristr., Cat. Coll. B. p. 43 (1889). - Meyer, Ibis, 1890, p. 422 (Gumbu, N. E. New Guines). - Salvad., Ann. Mus. Civ. Gen. (2) X, p 824 (1891) (Kunirira, Igibirei, Bujakori).

Ptilinopus (Megaloprepia) assimilis, Ramsay, Tab. List, p. 18 (partim) (1888).

\section{(764) Carpophaga myristicivora (Scop.).}

Carpophaga myristicivora, Nehrk., Journ. f. Orn. 1885, p. 34 (Waigeu). - Guillem., P. Z. S. 1885, p. 575 (Weeda Isl.), 659 (Waigiou, Batanta).

$?$ Carpophaga temmincki, Tristr. (nec Wall.), Cat. Coll. B. p. 43 (1889) (Waigiou, New Guinea).

La Carpophaga temminchi, Wall. è identica colla C.perspicillata (Temm.) del gruppo di Halmahera e di Bouru e quindi non so a quale specie si possano riferire gli esemplari di Waigiou e della Nuova Guinea dal Tristram attribuiti alla $C$. temmincki!

(765) Carpophaga rubricera, BP.

Carpophaga rubricera, Finsch, Vog. der Südsee, p. 18 (Nova Britannia) (1884)。 - Sharpe in Gould's B. New Guin. pt. XIX, pl. 7 (1885). - Tristr., Cat. Coll. B. p. 43 (1889) (New Hanover).

(766) Carpophaga rufigula, SALVAD.

Carpophaga (Globicera) rufigula, Ramsay, Pr. Linn. Soc. K. S. W. VIl, p. 36, 668 (1882) (Isole Salomone).

Carpophaga rufigula, Tristr., Ibis, 1882, p. 144. - Id., Cat. Coll. B. p. 43 (1889).

Globicera rufigula, Grant, P. Z. S. 1887, p. 332 (Malayta); 1888, p. 190 (Guadalcanar).

\section{(767) Carpophaga concinna, WaLL.}

Carpophaga concinna, Meyer, Verh. z.-b. Ges. Wien, 1881, p. 772 (Babbar, Cera, Tenimber). Sclat., P. Z. S. 1883, p. 51, 195, 200 (Larat, Maroe, Timorlaut) - Meyer, Sitzb. u. Abb. Gesellsch. Isis, 1884, Abh. I, p. 51 (Dammar, Riedel). - Forbes, P. Z. S. 1884, p. 433. - Büttik., Not. Leyd. Mus. VIIl, p. 66 (1886). - Tristr., Cat. Coll. B. p. 42 (1889) (Maur, Tenimber Islands).

(1) Ptilopus swainsoni, Gould.

Ptilinopus swainsoni, Ramsay, Tab. List, p. 17 (1888).

Il Ramsay, ma questa volta dubitativamente, torna ad annoverare la Nuova Guinea meridionale tra i luoghi abitati da questa specie Australiana. 
(770) Carpophaga pistrinaria, BP.

Garpophaga pristinaria (sic), Ramsay, Pr. Linn. Soc. X. S. W. VII, p. 35 (1883) (Isole Salomone). Carpophaga pistrinaria, Tristr., Ibis, 1882, p. 144. - Id., Cat. Coll. B. p. 42 (1889).

\section{(771) Carpophaga van-wyckii, OAsȘ.}

Carpophaga van-wyckii, Finsch, Vög. d. Südsee, p. 19 (Credner Island) (1884). - Sharpe in Gould's B. New Guin. pt. XXV, pl. 8 (1888). - Tristr., Gat. Coll. B. p. 43 (1889).

Lo Sharpe afferma recisamente che questa specie è distinta dalla C. pistrinaria, Bp. delle Isole Salomone, la quale cosa anche io aveva riconosciuto (Orn. Pap. III, p. 558); egli dice che nella $C$. pistrinaria le parti superiori sono più decisamento grigie e quasi non hanno lo splendore verde metallico che si osserva nella prima; inoltre nella $C$. pistrinaria il colore grigio della cervice e della regione interscapolare è appena diverso dal colore del dorso, laddove nella $C$. van-wyckii il colore grigio di quelle parti contrasta col colore del dorso, che è decisamente verde bronzato.

\section{(772) Carpophaga rosacea (TEмr.).}

Carpophaga rosacea, Sclat., P. Z. S., 1883, p. 51, 195, 200 (Loetoe, Maroe, Timorlaut). - Forb P. Z. S. 1884, p. 433. - Tristr., Cat. Coll. B. p. 42 (1889) (Timor).

(778) Carpophaga brenchleyi, G. R. Gr.

Carpophaga brechleyi, Ramsay, Pr. Linn. Soc. N. S. W. VII, p. 35 (1882) (Isole Salomone). Tristr., Ibis, 1882, p. 144. - Grant, P. Z. S. 1888, p. 186, 199 (Guadalcanar).

In certi tempi è comune; si nutre dei frutti di un fico.

Iride rossa, quasi rosso-sanguigna; piedi rossi.

(774) Carpophaga perspicillata (TEMr.).

Carpophaga perspicillata, Pleske, Bull. Acad. Petersb. XXIX, p. 535 (Ternate) (1884). - Tristr., Cat. Coll. B. p. 42 (1889). - Heine et Rchnw., Nomencl. Mus. Hein. Orn. p. 281 (1890 (NeuGuinea!).

(775) Carpophaga neglecta, Schleg.

Carpophaga negletta, Blas, P. Z. S. 1882, p. 709 (Ceram). - Meyer, Sitzb. U. Abh. Gesellsch. Isis, 1884, Abh. I, p. 51.

La lunghezza dell'ala di questa specie è di 285 millim. invece di 185 come per errore fu stampato a pag. 94 del Vol. III della Ornitologia.

\section{(776) Carpophaga zoeae (Less.).}

Carpophaga zoeae, Sharpe, Journ. Linn. Soc, Zool. XVl, p. 446 (Choqueri) (1882). - Guillem., P. Z. S. 1885, p. 659 (Aru). - Finsch u. Meyer, Zeitschr, f. ges. Orn. 1886, p. 27 (Monti Hufeisen). - Mejer, ibid. p. 37 (Kafu). - Tristr., Cat. Coll. B. p. 43 (1889). - Salvad., Ann. Mus. Civ. Gen. (2) X, p. 825 (1891) (Kunirira, Bujakori).

(777) Carpophaga basilica, Sund.

Garpophaga basilica, Pleske, Bull. Acad. Petersb. XXIX, p. 535 (Ternate) (1884). - Guillem., P. Z. S. 1885 , p. 575 (Batchian), - Tristr., Gat. Coll. B. p. 42 (1889).

Ducula basilica, Heine et Rchnw., Nomencl. Mus. Hein. Orn. p. 282 (1890) (Neu-Guinea!). 
(778) Carpophaga rufiventris, SALVAD.

Carpophaga rufigula (errore?), Bernst. (nec Salvad.) Dagboek, p. 139 (Sorong) (1883).

Carpophaga rufiventris, Nehrk, Journ. f. Orn. 1885, p. 34 (Waigeu). - Guillem., P. Z. S. 1885, p. 660 (Waigiou, Salwatti, Mysol, Andai, Batanta:

Carpophaga rufigastra, Tristr., Cat. Coll. B. p. 43 (1889).

Zonoenas rufiventris, Heine et Rchnw., Nomencl. Mus. Ilein. Orn. p. 282 (1890).

(1023) Carpophaga finschi, RaMsar.

Carpophaga finschi, Ramsay, Pr. Linn. Soc. N. S. W. VII, p. 43 (1882), - Id., Journ. Linn. Soc., Zool. XVII, p. 25 (1883). - Sharpe in Gould's B. New Guin. pt. XVII, pl. 2 (1884).

Questa specie, affine alla C. rufiventris, Salvad., si distingue facilmente per avere sulla coda una fascia cinerea che non è apicale, ma subapicale.

(779) Carpophaga chalconota, SaLvad.

Carpophaga chalconota, Guillem., P. Z. S. 1885, p. 660 (Arfak).

(780) Carpophaga muelleri (TEмr.).

Garpophaga muelleri, Sharpe, Journ. Linn. Soc., Zool. XVI, p. 446 (Taburi (1882). - Guillem. P. Z. S. 1885, p. 660 (Aru).

(781) Carpophaga pinon, Q. ot G.

Carpophaga pinon, Meyer, Zeitschr. f. ges. Orn. I, p. 294 (uova) (1884). - Nehrk., Journ. f. Orn. 1881, p. 34 (Waigeu). - Guillem. P. Z. S. 1885, p. 660 (Waigiou, Batauta, Mysol, Aru)。 - Tristr., Cat. Coll. B. p. $42(1889)$.

\section{Sp. 1157 (781 bis) Carpophaga rubiensis, MEYER.}

Carpophaga pinon, Sharpe (nec Q. et G.), Journ. Linn. Soc., Zool. XVII, p. 446 (Taburi) (1882). Carpophaga pinon var. rubiensis, Meyer, Sitzb. u. Abb. d. Ges. Isis, 1884, Abh. I, p. 51 (Rubi). Carpophaga rubiensis, Salvad., Ann. Mus, Civ. Gen. (2) IX, p. 588 (1890) (Rigo); X, p. 825 (1891) (Bujakori).

Carpophaga C. pinon similis, sed tectricibus alarum et subalaribus griseo marginatis et supracaudalibus obscurioribus, distinguenda.

Hab. in Nova Guinea - prope Rubi (Meyer) et in parte meridionali-orientali (Loria).

Ho esaminato due esemplari di questa specie raccolti dal Loria presso Rigo e presso Bujakori e ne ho confrontato uno con uno degli esemplari tipici, inviatomi cortesemente dal Meyer.

Questa specie si distingue facilmente dalla C. pinon pei margini grigio-chiari, non bianchi, come dice il Meyer, delle cuopritrici superiori ed inferiori delle ali, per la quale cosa quelle cuopritrici presentano un disegno a squame molto distinto; per quel carattere la C. mubiensis somiglia alla C. westermanni, Schleg. di Jobi; inoltre è da notare che le piume più lunghe del sopraccoda sono di color nero e sottilmente marginate di grigio come nella $C$. westermanni, e non, come nella $C$. pinon, di color grigio uniforme. La $C$. rubiensis è intermedia alle due specie menzionate, avendo il corpo, tranne il sopraccoda, colorito come la C. pinon, e le ali ed il sopraccoda come la C. vestermanni. 
(782) Carpophaga westermanni, RosENB.

Carpophaga westermanni, Meyer, Sitzb. u. Abh. Gesellsch. Isis, 1884, Abh. I, p. 51 (uovo). Id., Zeitschr. f. ges. Orn. 1886, p. 37 (Kafu), - Tristr., Cat. Coll. B. p. 43 (1889).

(1024) Carpophaga salvadorii, Tristr.

Carpophaga salvadorii, Tristr., Cat. Coll. B. p. 43 (1889).

(783) Carpophaga melanochroa, ScLat.

Carpophaga melanochroa, Sclat., P. Z. S. 1883, p. 348. - Tristr., Cat. Coll. B. p. 42 (1889).

Lo Sclater menziona altri due esemplari di questa rara specie.

(784) Myristicivora bicolor (Scor.).

Myristicivora melanura, part., Blas., P. Z. S. 1882, p. 709 (Ceram). - Bernst., Dagboek, pp. 127, 160 (Ramoi, Kalwal) (1883). - Nehrk. (nec Gray', Journ. f. Orn. 1885, p. 34 (Waigeu). - Salvad., Ibis, 1886, p. 153.

Myristicivora bicolor, Sclat., P. Z. S. 1883, pp. 51, 195, 200 (Kirimoen, Timorlaut). - Forb., P. Z. S. 1884, p. 433. - Guillem., P. Z. S. 1885, p. 575 (partim, Weeda Isl. 8).

Carpophaga bicolor, Büttik., Not. Leyd. Mus. VIII, p. 66 (1886). - Tristr., Cat. Coll. B. pp. 42, 269 (1889).

(785) Myristicivora spilorrhoa (G. R. GR.).

Carpophaga spilorrhoa, Meyer, Verh. z.-b. Ges. Wien, 1881, p. 773 (Aru). - Finsch, Vög. d. Südsee, p. 28, 39 (1884). - Guillem., P. Z. S. 1885, p. 661 (Aru). - Ramsay, Tab. List, p. 18 (1888). - Tristr., Ibis, 1889, p. 558. - Id., Cat. Coll. B. p. 43 (1889). - Salvad., Ann. Mus. Uiv. Gen. (2) IX, p. 588 (1890) (Rigo).

Hab. in Ins. St. Aignan dicta (Thomson).

Sp. 1158 (785 $5^{\text {bis }}$ ) Myristicivora subflavescens (FINscH).

?Carpophaga luctuosa, Ramsay (nec Reinw.), Pr. Linn. Soc. N. S. W. I, p. 373 (1876) (New Ireland and Duke of York Island); II, p. 376 (1878).

Carpophaga subflavescens, Finsch', Ibis, 1886, p. 2 (Nova Hibernia). - Sharpe in Gould's B. New Guin. pt. XXV, pl. 7 (1884).

Albo-flavescens, capite et gastraeo flavicantioribus ; remigibus nigris; subcaudalibus late nigro terminatis; dimidio apicali caudae nigro, nigredine sensim rectricem extimam versus, minus extensa, rachyde rectricum conspicue flava; iride brunnea; rostro virescente, apice flavo; pedibus plumbeis.

Long. tot. $0^{\mathrm{m}}, 355$; al. $0^{\mathrm{m}}, 228$; caud: $0^{\mathrm{m}}, 127$.

Hab. in Papuasia - Nora Hibernia (Finsch).

Dice il Finsch che questa specie si distingue immediatamente dalla $M$. spilorrhoa pel colorito distintamente giallognolo, per la timoniera esterna, che nella $M$. spitorrhoa è bianca quasi fino all'apice, e per le macchie nere all'apice delle piume del sottocoda marginate di bianco all'estremità.

Questa specie si troverebbe, ma non vi sarebbe abbondante, all'estremità settentrionale della Nuova Irlanda.

Anche il Ramsay (Pr. Linn. Suc. N. S. W. III, p. 293) affermò che la Myristicivora della Nuova Irlanda ed anche delle isole del Duca di York certamente non è la stessa della specie australiana, cioè della $M$. spilorrton. 
Io ho esaminato esemplari come quelli descritti dal Finsch, anche della Nuova Guinea e dubito the quelli della Nuova Irlanda non siano specificamente distinti. Secondo me, la $M$. subflavescens è fondata sopra esemplari della $M$. spilorrhoa, la cui muta è molto recente, e che perciò hanno le piume freschissime.

(786) Myristicivora melanura, G. R. GR.

Myristicivora melanura, Blas. u. Nehrk., Verh. z.-b. Ges. Wien, 1882, p. 429 (Amboina) - Blas. P. Z. S. 1882 , p. 710 (partim) (Ceram).

Myristicivora bicolor, Guillem. (nec Scop.) P. Z. S. 1885, p. 525 (Halmaheira). - Salvad., lbis, 1886, p. 153.

Carpophaga melanura, Tristr., Cat. Coll. B. p. 42 (1889) (Bouru).

\section{(787) Gymnophaps albertisi, SALVAD.}

Gymnophaps albertisi, Guillem., P. Z. S. 1885, p. 661 (Arfak, Jobi). - Finsch u. Meyer, Zeitschr. f. ges. Orn. 1886, p. 28 (Monti Hufeisen). - Meyer, Ibis, 1890, p. 423 (Constantine Harbour). - De Vis, Ann. Rep. Brit. New Guin. p. 62 (1890) (Gaesar Spur). - Id., Colon. Papers, no, 103, p. 116 (1890). - Id., Ibis, 1891, p. 41. - Salvad., Ann. Mus. Civ. Gen. (2) X, p. 825.

(1891) (Bujakori).

Jobi è una nuova località per questa specie ; il Guillemard dice che l'esemplare raccolto in quella isola differisce da quelli del Monte Arfak per avere il mento e la gola grigi anzichè castagno, questo colore essendo confinato alla regione auriculare; il suo petto è leggermente macchiettato di grigio, la quale cosa non si verifica negli altri.

\section{[Columba intermedia, Strickt.}

Columba intermedia, Pleske, Bull. Acad. Petersb. XXIX p. 535 (1884).

Il Pleske menziona un esemplare, ucciso in Ternate ed inviato dal Fisker, della Colomba domestica, ma inselvatico, e perciò la C. intermedia, Strickl. non merita di essere annoverata fra le specie proprie di Ternate.]

\section{(788) Ianthoenas albigularis, $\mathrm{Br}$.}

Ianthoenas albigularis, Pleske, Bull. Acad. Petersb. XXIX, p. 535 (Ternate) (1884). - Sharpe in Gould's B. New Guin. pt. XXV, pl. 6 (1888). - De Vis, Ann. Rep. Brit. New Guin. p. 62 (1890) (Mount Gleeson at 1600 feet). - Id., Colon. Papers, no. 103, p. 116 (1890). - Id, Ibis, 1891, p. 41. Carpophaga halmaheira, Tristr., Cat. Coll. B. p. 42 (1889).

Carpophaga albigularis, Tristr., ibid. p. 269 (1889).

Lo Sharpe, trattando di questa specie, non menziona l'esemplare delle Luisiadi, raccolto dal Mac Gilliwray; avendolo io esaminato recentemente nel Museo Britannico, mi sono assicurato che esso non differisce in alcun modo dagli esemplari delle altre località.

(1025) Ianthoenas philippanae, RAMSAT.

Ianthoenas philippanae, Ramsay, Pr. Linn. Soc. N. S. W. VII, pp. 38, 669 (1882) (Ugi, S. Cristoval).

Il collettore Stephens fa notare al Ramsay che alcuni degli esemplari raccolti hanno soltanto la gola bianca ed $\mathrm{i}$ piedi di color rosso cupo invece di giallo; il Ramsay ha considerato tali esemplari come giorani (!), e poscia come forse diversi specificamente. 
Gen, REINVARDTOENAS, Bp.

Coccyzoenas, Heine, Numencl. Mus. Hein. Ora. p. 278 (1890) (= Reinvardtoenas, Bp.).

\section{(790) Reinwardtoenas reinwardti (Tемm.).}

Reinwardtoenas reinwardti, Sharpe, Journ. Linn. Soc., Zool. XVI, p. 446 (Taburi, Milne Bay) (1882). - Blas. u. Nehrk., Verh. z.-b. Ges. Wien, 1882, p. 429 (Amboina). - Nehrk., Journ. f. Orn. 1885, p. 34 (Waigeu). - Guillem., P. Z. S. 1885, p. 376 (Batchian, Obi Latu), p. 661 (Waigiou, Batanta). - Finsch u. Meyer, Zeitschr. f. ges. Orn. 1886, p. 28 (Nonti Hufeisen). - Wardl, Rams, Ibis, 1890, p. 241. - Meyer, Ibis, 1890, p. 423 (Constantine Harbour).

IMacropygia reinwardti, Tristr., Cat. Coll. B. p. 39 (1889).

Goccyzoenas reinwardti, Heine et Bchnw., Nomencl. Mus. Hein. Orn. p. 278 (1890).

(791) Reinwardtoenas minor (ScHLEG.).

Reinwardtoenas minor, Wardl. Rams., Ibis, 1890, p. 242.

\section{(792) Reinwardtoenas browni (ScLat.).}

Reinwardtoenas browni, Selat., P. Z. S. 1883, p. 348. - Wardl. Rams., Ibis, 1890, p. 242. Macropygia browni, Tristr., Cat. Coll. B. p. 39 (1889).

Io aveva già riconosciuto (Orn. Pap. e Mol. II, p. 561) che la Turacoena crassirostris, Gould, delle Isole Salomone non è il giovane di questa specie.

GEN. GORYPHOENAS, WARDL. RAMS.

Typus:

Coryphoenas, Wardl. Rams., Ibis, 1890, p. 246 . . . . Turacoena crassirosiris, Gould.

(793) Coryphoenas crassirostris (GooLD).

Turacoena crassirostris, Ramsay, Pr. Linn. Soc. N. S. W. VII, p. 36 (1882) (Isole Salomone). - Haswell, ibid. p. 116 (1882) (anatomia). - Grant, P. Z. S. 1888, p. 199 (Aola, Guadalcanar).

Macropygia crassirostris, Tristr., Ibis, 1882, p. 144. - ld., Cat. Coll. B. p. 269 (1889).

Coryphoenas crassirostris, Wardl. Rams, lbis, 1890, p. 246.

(794) Macropygia amboinensis (LinN.).

Macropygia amboinensis, Blas. u. Nehrk., Verh. z.-b. Ges. Wien, 1882, p. 429 (Amboina). Tristr., Cat. Coll. B. p. 39 (partim) (1889) (Amboina) - Wardl. Rams., 1890, p. 231. - ? Heine et Rchnw., Momencl. Mus. Hein. Orn. p. 278 (1890) (Gilolo!, Celebes !)

Il Wardlaw Ramsay è d'opinione che la mia $M$. buruensis di Buru non sia diversa specificamente dalla $\boldsymbol{M}$. amboinensis, giacchè egli afferma che il numero delle fascie scure delle piume del petto varia tanto negli esemplari di Amboina, quanto in quelli di Buru.

\section{(796) Macropygia batchianensis, WALL.}

Macropygia batchianensis, Pleske, Bull. Acad. Petersh. XXIX, P. 535 (Ternate) (1884). - Guil. lem., P. Z. S. 1885 , p. 576 (Ternate). - Wardl. Rams., Ibis, 1890, p. 237. - ? Ileine et Rchnw., Nomencl. Mus. Hein. Orn. p. 278 (1889) (Mysol !).

Macropygia amboinensis (part.), Tristr., Cat. Coll. B. p. 39 (1889) (Batchian).

\section{(797) Macropygia carteretia, BP.}

Macropýgía carteretia . Tristr., Ibis, 1889, p. 558. - IJ., Cat. Coll. B. p. 39 (1889). - WardI. Rams., Ibis, 1890, p. 238.

$[60]$ 
Macropygia nigrirostris, De Vis (nec Salvad.), Ann. Rep. Brit. New Guin. p. 62 (1890) (St. Aignan, Russel Island). - Id., Colon. Papers, no. 103, p. 116 (1890). - Id., lbis, 1891, p. 41.

$H a b$. in Ins. St. Aignan dicta (Thomson); insula Rossel (fide $D e V i s$ ).

Il tipo della Mricropygia carteretia è della Nuova Irlanda, e non so se gli esemplari delle Isole Luisiadi si possano riferire alla medesima specie.

(798) Macropygia griseinucha, BALPAD.

Macropygia griseinucha, WardI. Rams, Ibis, 1890, p. 238.

\section{(799) Macropygia doreya, Br.}

Macropygia doreya, Ramsay, Pr. Linn. Soc. N. S. W. VIII, p. 27 (1883) (uova). - Nehrk., Journ. f. Orn. 1885, p. 34 (Waigeu). - Tristr., Cat. Coll. B. p. 39 (1889. - Wardl. Ramsay, Ibis, 1890, p. 234 .

\section{Macropygia, sp.}

Macropygia sp., Meyer, Zeitschr. f. ges, Orn. 1884, p. 215 (Aru).

Il Mejer fa notare che una Macropygia raccolta in Dobbo nelle Isole Aru dal Riedel differisce dalla $M$. doreya, Bp., da me (Orn. III, p. 141) annoverata fra le specie delle isole Aru, per essere più grande (ala $180 \mathrm{~mm}$. invece di 155-165 mm., coda $215 \mathrm{~mm}$. invece di 180-190 mm.); inoltre le parti superiori sono più oscure, i margini delle cuopritrici delle ali meno rossigni e più sottili, la testa sottilmente fasciolata, non unicolore e le parti inferiori di color rossigno-cannella uniforme; essa sarebbe quindi da confrontare colla $M$. phasianella, T. e colla $M$. amboinensis, $\mathrm{L}$. Aggiunge il Meyer che l'esemplare delle isole Aru somiglia, tranne che nelle dimensioni, ad un altro di Rubi nella Nuova Guinea.

\section{(800) Macropygia keyensis, SALVAD.}

Macropygia keyensis, Wardl. Rams., Ihis, 1890, p. 236.

\section{Sp. 1159 ( $800^{\text {tis }}$ ) Macropygia timorlaoënsis, MEYER.}

Macropygia sp inc., Sclat., P. Z. S. 1883, p. 51, 195, 200.

Macropygia keiensis, Meyer (nec Salvad.), Sitzb. u. Abb. Gesellsch. Isis, 1884, p. 52. - Forb., P. Z. S. 1884, p. 433.

Macropygía timorlaoënsis, Meyer, Zeitschr, f. ges, Orn. 1884, p. 214 (Timor-laut). - Bültik., Not. Leyd. Mus. VIII, p. 46 (1886). - Wardl. Rams., Ibis, 1890, p. 240.

Macropygia sp., Tristr., Cat. Coll. B. p. 39 (1889) (Timor-laut).

Capite brunnescenti rufo et nigro, collo postico albescenti et nigro, paulluni viridi et violaceo nitente transfasciolatis; dorso, uropygio, supracaudalibus brunnescenti-fulvis, punctuTis albescentibus obsoletis adspersis; gula alba; collo antico et pectore plus minusve albo, nigro et rufescenti transfasciatis, plumarum basi cinerea, abdomine pallidiori vel rufescentiori; subcaudalibus pallide cinnamomeis, fusco transfasciolatis vel vermiculatis; subalaribus cinnamomeis, fusco-nigro notatis; axillaribus unicoloribus cinnamomeis, remigibus fuscis, exterius anguste albescenti et brunnescenti limbatis; alarum tectricibus cinnamomeo transfasciatis; remigum pogonio interno subtus cinnamomeo marginato; cauda superne brunne- 
scenti-fulva, inferne pallidiore et magis grisescente; rectricibus extimis pogonio interno in medio cinnamomeo et macula stibapicali nigra notatis; rostro brunneo.

Long. tot. $0^{\mathrm{m}}, 370-0^{\mathrm{m}}, 380$; alt. $0^{\mathrm{m}}, 195-0^{\mathrm{m}}, 200$; caud. $0^{\mathrm{m}}, 190-0^{\mathrm{m}}, 200$; rostri culm. $0^{\mathrm{m}}, 024-0^{\mathrm{m}}, 026$; tarsi $0^{\mathrm{m}}, 020-0^{\mathrm{m}}, 021$.

Hab. in Papuasia - Ins. Timor-laut (Riedel).

Il Meyer avera da prima riferito gli esemplari di Timor-laut alla Macropygia Keiensis, Salvad., ma egli ha fatto notare come, dopo l'arrivo di una più numerosa serie di esemplari, abbia dovuto riconoscere che i primi non erano sufficienti per la determinazione della specie, e che gli esemplari di Timor-laut non possono essere attribuiti alla $M$. Keiensis, dalla quale differiscono per le dimensioni delle ali ; (nella $M$. keiensis lunghe $180 \mathrm{~mm}$. e nella $M$. timorlaoensis lunghe da $195-200 \mathrm{~mm}$.), per la coda più lunga ed inoltre pel diverso colorito. Le grandi cuopritrici della coda non sono unicolori, e principalmente diverso è il disegno delle macchie nelle timoniere esterne, pel quale rispetto la $M$. timorlaoensis differisce da tutte le altre specie, le quali hanno le 3 a 4 timoniere esterne più o meno variegate, mentre nella $M$. timorlaoensis soltanto la prima, od anche la seconda hanno una lieve traccia di macchia nera sul vessillo interno; finalmente le parti inferiori non sono bianchiccie, e la testa non unicolore.

Aggiunge il Mejer che nella $M$. timorlaoensis tanto le parti superiori, quanto le inferiori sono tutte dotate di fascie trasversali, mentre nelle altre specie lo sono soltanto in parte; finalmente la $M I$. timorlaoensis è in generale più scura e meno rariegata.

Il Büttikofer, che ha ricevuto un solo maschio adulto di questa specie, fa notare che essa è affine al $M$. magna, Wall. di Timor, Wetter e Lettie per le sue dimensioni e pel modo di fasciatura su tutte le parti, eccetto la coda; si può tuttavia distinguerla immediatamente pel colore della coda, che è bruno-grigiastra, e non fulva superiormente e rossigna inferiormente.

(801) Macropygia maforensis, SALVAD.

Macropygia maforensis, Wardl. Rams, Ibis, 1890, p. 236.

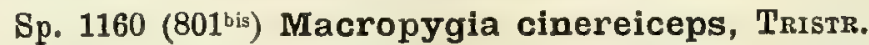

Macropygia cinereiceps, Tristr., Ibỉe, 1889, p. 558. - Ward!. Rams., Ibis, 1890, p. 237.

« II. corpore superne cinnamomeo; capite cinereo; mucha viridi resplendente; mento albicante; pectore rufo-cinnamomeo; abdomine albidiore; subcaudalibus larle castaneis; remigibus brunneis, castaneo marginatis; cauda castanea; rectricibus tribus externis fascia lata nigricante in medio cinctis » (Tristram).

Hab. in Ins. Fergusson dicta (Thomson); in Nova Guinea meridionali-orientali (Forbes).

Dice il Tristram che il colore cenerino della testa caratterizza abbastanza questa specie, il tipo della quale evidentemente è un esemplare al tutto adulto; egli aggiunge che un secondo esemplare di questa specie, raccolto dal Forbes nella Nuova Guinea meridionale-orientale, si conserva nel Museo Britannico.

[62] 
(802) Macropygia rufocastanea, RAMSAY,

Macropygia rufocastanea, Ramsay, Pr. Linn. Soc. N. S. W. VII, pp. 37, 673 (1882) (Isole Salomone..-Grant, P. Z. S. 1887, p. 332 (Alu); 1888, p. 200 (dola). - WardI. Rams., Ibis, 1890, p. 229. Macropygia arossiana, Tristr., Cat. Coll. B. p. 39 (1889).

\section{(803) Macropygia nigrirostris, Saltad.}

Macropygia nigrirostris, Finsch, Vög. d. Südsee, p. 19 (Nova Britannia) (1884) - Guillem., P. Z. S. 1885, p. 662. - Wardl. Rams., Ibis, 1890, p. 230. - Meyer, Ibis, 1890, p. 423 (New Britain). - Heine et Rchnw., Nomencl. Mus. Hein. Orn. p. 278 (1890 _- Salvad . Ann. Mus. Civ. Gen. (2) X, p. 825 (1891) (Igihirei, Kunirira).

\section{(804) Spilopelia tigrina (Темм.).}

Spilopelia tigrina, Meyer, Verh. z.-b. Ges. Wien, 1881, p. 766 (Sumba); 769 (Wetter); 772 (Da: weloor). - Id., Zeitschr. f. ges. Orn. I, pp. 195, 215 (1884) (Timorlaut, Riedel).

\section{(805) Geopelia tranquilla, Govrd.}

Geopelia tranquilla, Finsch, Vög. d. Südsee, p. 29 (Port Moresby), 39 (1884). - Ramsay, Tab. List, p. 18 (1888). - Salvad, Ann. IJus. Civ. Gen. (2) IX, p. 589 (1890); X, p. 826 (1891).

Geopelia placida, Hamsay, I. c. - Tristr., Cat. Coll. B. p. 41 (1889).

(806) Geopelia striata (Linn.).

Geopelia striata, Meyer, Vehr. \%.-b. Ges. Wien, 1881, p. 772 (Babbar, Daweloor). - Tristr., Cat. Coll. B. p. 41 (1889).

\section{(807) Goepelia maugei (Tems.).}

Geopelia maugei, Sclat., P. Z. S. 1883, pp. 51, 195, 200 (Larat, Loetoe, Timorlaut). - Forb.; P. 7. S. 1884, p. 433. - Büttik., Not. Leyd. Mus. vilI, p. 67 (1886). - Tristr., Cat. Coll. B. p. 41 (1889) (Flores'.

(808) Erythrauchoena humeralis (Tемм.).

Geopelia humeralis, Ramsay, Tab. List, p. 18 (1888).

Erythrauchoena humeralis, Salvad, Ann. Mus. Civ. Gen. (2) IX, p. $589(1890)$.

(809) Phlogoenas rufigula (PסCH. et $\mathrm{J}_{\mathbf{\Lambda} \mathrm{CQ} .}$ ).

Phlogoenas rufigula, Guillem., P. Z. S. 1885 , p. 662 (Salwatti, Arfak). - Tristr, Cat. Coll. B. p. 41 (partim) (1889;

\section{(810) Phlogoenas helviventris (Rosens.).}

Phlogoenas rufigula, Ramsay (nec P. et J.), Pr. Linn. Soc. N. S. W. VIII, p. 15 (1883). - Tristr., Cat. Coll. B. p. 41 (partim) (1889\%.

Phlogoenas helviventris, Finsch u. Mey., Zeitschr. f. ges. Orn. 1886, p. 28 (Monti Hufeisen).

\section{(811) Phlogoenas joblensis, Meyer.}

Phlogoenas jobiensis, Sharpe, Journ. Linn. Soc., Zool. XVI, p. 446 (Choqueri) (1882). - Finsch u. Meyer, Zeitschr. f. ges. Orn. 1886, p. 28 (Monti Hufeisen). - Tristr., Cat. Coll. B. p. 269 (1889). - Salvad., Ann. Mus. Civ. Gen. (2) IX, p. 589 (1890) (Rigo).

Chalcophaps Margaritae, Finsch, Vög. d. Sủdsee, p. 19 (Nova Britannia) (1884).

Plegoenas margarethae, Tristr., Cat. Coll. B. p. 41 (1889) (Duke of York Islands).

\section{Phlogoenas $\mathrm{sp}$.}

Phlogoenas spec. inc., Grant, P. Z. S. 1888, p. 201. 
L'Ogilvie-Grant annovera tre giovani esemplari, tutti maschi, di Aola, Guadal. canar, appartenenti ad una specie non ancora descritta, ma affine alla $P h$. jobiensic, Meyer (= Ph. margaritae, D’Alb. et Salrad.), dal giovane della quale, egli dice che quelli differiscono per mancare della stria sopraccigliare bianca, la quale cosa non è esatta, giacchè in un individuo conservato nel Tuseo Britannico, sulle redini appare un principio della fascia sopraccigliare. Il Grant afferma che evidentemente essi appartengono alla stessa specie che il Ramsay (Pr. Linn. Soc. N. S. W. I, p. 374, 1876) identificò dubitativamente colla $P$ h. margaritae (D'Alb. et Salvad.).

A me pare che in questa ultima asserzione dell'Ogilvie-Grant vi sia un errore, giacchè gli esemplari menzionati dal Ramsay erano delle Isole del Duca di York e non delle Isole Salomone.

L' esemplare del Museo Britannico è certamente diverso dalla $P$. jobiensis, avendo $\mathrm{i}$ piedi notevolmente più grandi.

(1026) Phlogoenas salomonis, Ramsay.

?Phlogoenas johannae, Ramsay (nec Sclat.), Pr. Linn. Soc. N. S. W. VII, p. 38 (1882). - Tristr., Cat. Coll. B. p. 41 (1889) (San Christoval).

Phlogoenas salomonensis, Grant, P. Z. S. 1888, pp. 186, 200 (Aola, Guadalcanar).

Phlegoenas sp., Tristr., Cat. Coll. B. p. 269 (1889) (Guadalcanar).

L'Ogilvie-Grant reeentemente è tornato a descrivere questa specie, ma non soperchè, come nuova! Egli fa notare come la medesima sia affine alla $P h$. johannae, Sclat., ma ne differisca pel colorito più cupo, mentre la $P$. johannae, che ora si sa essere propria della Nuova Britannia, ha il petto grigio bianchiccio, e l'occipite, il dorso, ecc., tinti di olivaceo, anzichè di violaceo.

Ho esaminato il tipo descritto dall'Ogilvie-Grant e ad onta di certe differenze fra esso e la descrizione del Ramsay non dubito che si tratti della medesima specie

\section{(814) Ghalcophaps chrysochlora (WAGL.).}

Chalcophaps chrysochlora, Sclat., P. Z. S. 1883, pp. 51, 195, 200 (Larat, Moloe, Timorlaut). Forb., P. Z. S. 1884, p. 433. - Finsch u. Meyer, Zeitschr. f. ges. Orn. 1886, p. 28 (Monti Hufeisen). - Tristr., Ibis, 1889, p. 558. - Id., Cat. Coll. B. p. 41 (1889). - Salvad., Ann. Mus. Giv. Gen. (2) $1 X, p .589$ (1890) (Rigo).

Ghalcophaps indica, Bütlik. (nec Linn. ?), Not. Leyd. Mus. VIII, p. 67 (1886).

Hab. in Ins. East dicta (Thomson).

(815) Chalcophaps indica (LiNn.).

Chalcophaps indica, Pleske, Bull. Acad. Petersb. XXIX, p. 535 (Ternate) (1884). - Tristr., Cat. Coll. B. pp. 41, 269 (1889).

\section{(816) Chalcophaps stephani (PuCH. et JACQ.).}

Chalcophaps stephani, Ramsay, Pr. Linn. Soc. N. S. W. VII, p. 37 (1882) (Isole Salomone?). Finsch, Vög. d. Südsee, p. 19 (Niova Britannia) (1884). - Pleske, Bull. Acad. Petersb. XXIX, p. 535 (Ternate) (1884). - Nehrk., Journ. f. Orn. 1885, p. 34 (Waigeu). - Neyer, Zeitschr. f. ges. Orn. 1886, p. 37 (Kafu). - Grant, P. Z. S. 1888, p. 201 (Aola, Guadalcanar). - Tristr., Cat. Coll. B. p. 41 (1889).

(1027) Chalcophaps mortoni, RAMSAY.

Calcophaps mortoni, Ramsay, Pr. Linn. Soc. N. S. W. VIII, p. 37, 667 (1882) (Isole Salomone). $\lceil 64\rceil$ 
Il Ramsay, il quale affermò che questa specie è più grande della Ch. chrysochlora e che ne differisce per non avere la fascia bianca sulle cuopritrici delle ali, da ultimo ha riconosciuto che essa è molto affine alla $C$. stephani, come io areva supposto, se pure ne è diversa; egli dice di averne visti, oltre il tipo, cinque esemplari adulti.

Questa specie dovrà essere ulteriormente studiata.

(817) Henicophaps albifrons, G. R. GR.

Henicophaps albifrons, Sharpe, Journ. Linn. Soc., Zool. XVI, p. 446 (Taburi, China Straits) (1882). - Nehrk., Journ. f. Orn. 1885, p. 34 (Waigeu). - Guillem., P. Z. S. 1885, p. 662 (Waigiou). - Finseh u. Meyer, Zeitsehr. f. ges. Orn. 1886, p. 28 (Monti Hufeisen). - Tristr., Cat. Coll. B. p. 40 (1889). - Salvad, Ann. Mus. Civ. Gen. (2) X, p. 826 (1891) (Igibirei).

(818) Eutrygon terrestris (G. R. GRo).

Eutrygon terrestris, Guillem., P. Z. S. 1885, p. 662 (Andai). - Tristr., Cat. Coll. B. p. 40 (1889).

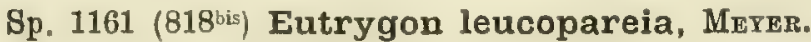

Eutrygon terrestris, partim, Sharpe in Gould's B. New Guin. pt. XIII (1882). - Salvad., Orn. Pap. e Mol. III, p. 563 (partim) (1882.

Eutrygon leucopareia, Meyer, Zeitschr. f. ges. Orn. 1886, p. 29.

Eutrygon Eu. terrestri (Gr.) similis, sed tectricibus alarum mediis et parvis rufescentibus vel rufescenti limbatis et genis, gutture sincipiteque albidioribus.

Long. tot. $0^{\mathrm{m}}, 300$; al. $0^{\mathrm{m}}, 170$; caud. $0^{\mathrm{m}}, 130$; rostri culm. $0^{\mathrm{m}}, 017$, tarsi $0^{\mathrm{m}}, 041$.

Hab. in Papuasia - Nova Guinea meridionali-orientali in Montibus Astrolabii (Goldie, Hunstein)

Il Meyer, fondandosi sul confronto di un esemplare dei Monti dell'Astrolabio con una serie d'individui della Nuova Guinea settentrionale, afferma che, oltre alle differenze sopraindicate, quello ha il becco più corto e più debole.

Ho esaminato nel Museo Britannico tre esemplari della Nuova Guinea meridionale, uno di Port Moresby, uno dei Monti dell'Astrolabio ed il terzo del Fiume Fly, e tutti tre presentano la tinta rossigna o porporina sulle cuopritrici minori e medie delle ali; non ho trovato differenza sensibile nella lunghezza del becco.

\section{(819) Otidiphaps nobilis, GouLd.}

Otidiphaps nobilis, Meyer, Sitzb. u. Abh. Gesellsch. Isis, 1884, Abh. I, p. 52 (uovo e nido). Guillem., P. Z. S. 1885, p. 662 (Arfak, Dorei ?). - Tristr., Cat. Coll. B. p. 40 (1889).

\section{(820) Otidiphaps cervicalis, RAMBAY.}

Otidiphaps cervicalis, Rams., Pr. Linn. Soc. N. S. W. VIII, pp. 16, 27 (1883) (nido ed uovo). Finsch u. Meyer, Zeitschr. f. ges. Orn. 1886, p. 29 (Monti Hufeisen). - Salvad., Ann. Mus. Civ. Gen. (2) X, p. 826 (1891) (Bujakori).

Otidiphaps regalis, Tristr., Cat. Coll. B. p. 40 (1889).

Sp. 1162 (820 $\left.{ }^{\text {bis }}\right)$ Otidiphaps insularis, S. ot G.

Otidiphaps insularis, Salvin et Godman, P. Z. S. 1883, p. 33.

* Capite toto, cervice et corpore subtus nigris purpurescente tinctis ; intersca- 
pulio, remigibus secundariis et tectricibus alarum omnibus pure cinnamomeis; remigibus fuscis; cauda nigra; dorso postico viridescenti-nigro, dorso imo et uropygio purpurascente tinctis ; rostro corallino-rubro; pedibus vinaceo-rubris, flavo squamulatis ».

Long. tot. $0^{\mathrm{m}}, 381$; al. $0^{\mathrm{m}}, 188$; caud. rectr. med. $0^{\mathrm{m}}, 167$; rostri a rictu $0^{\mathrm{m}}, 029 ;$ tarsi $0^{\mathrm{m}}, 066$.

Hab. in Papuasia - Ins. Fergusson dicta ad oras Novae Guineae orientalis (Goldie).

«Obs. Ab O. nobiti crista occipitali et macula nuchali absentibus, colore dorsi purius cinnamomeo nec purpureo tincto, colore dorsi postici viridescente nec omnino purpureo et cauda breviore diversus. Ab O. cervicali macula nuchali absente, colore dorsi purius cinnamomeo nec purpureo tincto et colore dorsi imi et uropygii purpureo distinguendus» (Salvin et Godman).

Il Goldie raccolse due esemplari di questa specie nei monti dell'Isola Fergusson, una del gruppo delle D'Entrecasteaux, ad un'altezza superiore ai 2000 piedi; egli nota che il suo grido è una sorta di ki-o, coll'o prolungato, che uno degli individui fu ucciso posato sopra un ramo basso di un grosso albero, e che l'iride era di color rosso e le gambe rosso-vinacee colle squame di color giallo-verdognolo chiaro.

Ho esaminato il tipo di questa specie nel Museo Britannico.

\section{(821) Goura coronata (LinN.).}

Goura coronata, Nehrk., Journ. f. Orn. 1885, p. 34 (Waigeu). - Guillem., P. Z. S. 1885, p. 662 (Waigiou, Mysol, Dorei).

Megapelia coronata, Heine et Rchnw., Nomencl. Mus. Hein. Orn. p. 287 (1890).

(822) Goura sclateri, SaLVAD.

Goura sclateri, Salvad., Ibis, 1885, p. 356.

? Goura albertsii (sic), Tristr. (nec Salvad.), Lat. Coll. B. p. 37 (1889) (Fly River).

Se l'esemplare menzionato dal Tristram è veramente del Fiume Fly, esso devo essere riferito alla $G$. sclateri.

(823) Goura albertisil, SALVAD.

Goura albertisii, Ramsay, Pr. Linn. Soc. N. S. W. VIII, p. 26 (1883)(nova). - Salvad., Ann. Mus. Giv. Gen. X, p. 826 (1891) (Igibirei).

Goura scheepmakeri, Finscb, Vög. d. Südsee, p. 29 (Port Moreshy) (1884) (nec 1875). - Salvad, Ibis, 1884, p. 355.

Goura albertisii, Salvad., ibid. p. 356, - Id., Ann. Mus. Civ. Gen. (2) IX, p. 590 (1890) (Calo).

(824) Goura scheepmakeri, Frnsch.

Goura scheepmakeri, Salvad., Ibis, 1884, p. 350.

(825) Goura victoria (Fraser).

Goura victoria, Meyer, Sitzb. u. Abh. Gesellsch. Isis, 1884, Abh. I, p. 52 (uovo). - Guillem., P. 2. S. 1885 , p. 663 (Jobi). 
(826) Goura beccarii, SaLvad.

Goura victoriae var. comata, Oust, Ann. Sc. Nat., Zool. 1885, Art. 3, p. 3 (Kafou)

Goura beccarii, Meyer, Zeitschr. f. ges. Orn. 1886, p. 17 (Kafu).

Goura G. victoriae (Fras.) similis, sed magis robusta, pedibus robustioribus, digitis longioribus, crista magis copiosa e plumis ad apicem spatulatum latioribus, colore griseo-caenulescente plumarum pallidiore, et plumis dorsi vix marginatis.

Long. digit. medii sine ungue ultra 50 millim.; lat. spatulae plumarum cristae interdum 40 millim.

Hab. in Papuasia - Nova Guinea septentrionali, prope Kafu.

Il Meyer fa notare come sia molto probabile che la Goura di Kafu sia la stessa di quella della Baia Humboldt, essendo i due luoghi molto vicini fra loro, e che la tinta rossigna sul margine apicale della spatola delle piume del ciuffo dell'esemplare tipico della mia Goura beccarii sia artificiale, la quale cosa io avera già sospettata, e quindi il Meyer non esita a riferire a questa specie la $G$. victoriae var. comata, Oust. Questa opinione del Meyer viene avvalorata dall' esame fatto nuovamente di quel ciuffo, che è realmente copioso e costituito di piume più lunghe e più largamente spatolate di quelle della Goura victoria.

\section{(827) Caloenas nicobarica (LiNv.).}

Callaenas nicobarica, Ramsay, Pr. Linn. Soc. N. S. W. VII, p. 38 (1882) (Ugi, Isole Salomone). Galoenas nicobarica, Sharpe, Journ. Linn. Soc., Zoọl. XVI, p. 446 (Milne Bay) (1882). - Meyer, Sitzb. u. Abh. Gesellsch. Isis, 1884, Abh. I, p. 52 (Siao, Sangi, Meyer). - Guillem., P. Z. S. 1885, p. 576 (Bisa Isl., Obi), 663 (Waigiou, Salwatti). - Grant, P. Z. S. 1887, p. 332 (Alu). - Woodford, P. Z. S. 1888, p. 248. - Tristr., Ibis, 1889, p. 558. - Id., Cat. Coll. B. p. 41 (1889).

Hab. in ins. Normanby (Thomson). 


\section{O R D 0 G A L L I N A E}

(828) Megapodius duperreyi, Less. et GARs.

Megapodius duperreyi, Meyer, Vehr. z.-b. Ges. Wien, 1881, p. 767 (Sumba). - Sharpe, Journ.

Linn. Soc., Zool. XVI, p. 447 (Choqueri) (1882). - Meyer, Zeitschr. f. ges. Orn. I, p. 294 (1884).

- Sharpe, Report Alert, Birds, p. 26 (1884). - Guillem., P. Z. S. 1885, p. 663 (Dorei). - Tristr.,

Cat. Coll. B. p. 30 (1889). - Salvad., Ann. Mus. Civ. Gen. (2) IX, p. 590 (1890) (Rigo).

Sp. 1163 (830is) Megapodius tenimberensis, ScLAt.

Megapodius tenimberensis, Sclat., P. Z. S. 1883, pp. 54, 57, 200. - Forbes, P. Z. S. 1884, p. 433.

Supra brunnescenti-olivaceus, in cervice magis cinereus, in dorso postice magis brunnescens; pileo subcristato interscaputio concolore; subtus cineraceus alivaceo tinctus; capitis lateralis et gulae pelle rubra plumis paucis obsita; subalaribus ventre concoloribus; rostro flavo; tarsis antice nigris postice rubris; digitis nigris.

Long. tot. $0^{\mathrm{m}}, 292$; al. $0^{\mathrm{m}}, 243$; caud. $0^{\mathrm{m}}, 089$; tarsi $0^{\mathrm{m}}, 071$.

Hab. in Papuasia - Ins. Tenimber dictis Kirimoen et Loetoe (Forbes).

Obs. Species pedum colore ad $M$. geelvinkianum, corporis pictura magis ad $M$. tumulum appropinquans. (Sclater).

(829) Megapodius macgillivrayi, G. R. GR.

Megapodius macgillivrayi, Tristr., Cat. Coll. B. p. 30 (1889).

(831) Megapodius freycineti, Q. et G.

Megapodius freycineti, Pleske, Bull. Acad. Petersb. XXIX, pp. 536, 540 (Ternate) (1884), - Nehrk., Journ. f. Orn. 1885, p. 34 (Waigeu). - Guillem., P. Z. S. 1885, pp. 576 (Batchian), 663 (Waigiou). - Tristr., Cat. Coll. B p. 30 (1889) (Kaysa, Mysol).

(832) Megapodius forsteni, TeMM.

Megapodius forsteni, Blas. u. Nehrk., Verh. z.-b. Ges. Wien, 1882, p. 22 (Amboina, uova). Blas., P. Z. S. 1882, p. 710 (Ceram). - Tristr., Cat. Coll. B. p. 30 (1889) (Bouru).

(884) Megapodius eremita, HARTL.

Megapodius eremita, Finsch, Vög. d. Südsee, p. 20 (Nova Britannia) (1884). - Meyer, Ibjs, 1890 , p. 423 (eggs).

Megapodius hueskeri, Tristr., Cat. Coll. B. p. 30 (1889).

(895) Megapodius brenchleyi, G. R. GR.

Megapodius brenchleyi, Brazier, Pr. Linn. Soc. N. S. W. VI, p. 150 (1881). - Ramsay, Pr. Linn. Soc. N. S. W. VII, p. 39,(1882) (Isole Salomone), - Sharpe in Gould's B. New Guin. pt. XXII, pl. 11 (1886). - Grant, P. Z. S. 1887, p. 332; 1888, p. 201. - Woodford, P. Z. S. 1888, p. 249. - Meyer, Ibis, 1890, p. 423 (egg).

[68] 
(836) Megapodius wallacei, G. R. GR.

Megapodius wallacei, Blas. u. Nehrk., Verh. z.-b. Ges. Wien, 1882, p. 430 (Amboina, uova). Pleske, Bull. Acad. Petersb. XXIX, p. 540 (Ternate) (1884).

(837) Talegallus jobiensis, MEYER.

Talegallus jobiensis, Guillem., P. Z. S. 1885, p. 663 (Jobi). - Meyer, Ibis, 1890, p. 424 (eggs).

(838) Talegallus cuvieri, Lrss.

Talegallus cuvieri, Guillem., P. Z. S. 1885, p. 664 (Salwatti, Dorei'.

Tallegalla cuvieri, Tristr., Cat. Coll. B. p. 30 (1889).

(899) Talegallus fuscirostris, SALVAD.

Talegallus fuscirostris, Meyer, Sitzb. u. Ahh. Gesellsch. Isis, 1884, Abh. I, p. 52 (uovo). - Salvad., Ann. Mus. Civ. Gen. (2) IX, P. 590 (1890) (Rigo). - Meyer, Ibis, 1890, p. 424 (egg).

(840) Aepipodius arfakianus, Salvad.

Talegallus pyrrhopygius, Ramsay, Pr. Linn. Soc. N. S. W. VIII, p. 26 (1883) (novo).

? Talegallus (sive Aepipodius) sp. inc., Meyer, lbis, 1890, p. 423 (eggs, Constantine Harbour).

(842) Synoecus cervinus, Govld.

Synoecus cervinus, Ramsay, Tab. List, p. 19 (1888).

(843) Excalfactoria minima, Gourd.

Excalfactoria minima, Pleske, Bull. Acad. Petersb. XXIX, p. 536 (Ternate) (1884).

(844) Excalfactoria lepida, HARTL.

Excalfactoria lepida, Finsch, Vơg. d. Südsee, p. 20 (Kova Britannia) (1884). - Tristr., Cat. Coll. B. p. 34 (1889) (1).

(846) Turnix melanonota (GouLD).

Hemipodius maculosus, Temm., Pig. et Gall. III, pp. 631, 757 (1815).

Turnix maculosus, Steph., Gen. Zool. XI, p. 394 (1819).

Turnix maculatus, Vieill., N. D. XXXV, p. 47 (1819). - Id., Enc. Méth. I, p. 530 (1823). - Id., Gal. des Ois. II, p. 51, pl. 217 (1825).

Turnix melanonotus, Ramsay, Tab. List, p. 18 (1888).

Turnix maculosa, Grant, Ibis, 1889, p. 468.

L'Ogilvie-Grant crede che la specie conosciuta generalmente col nome di T. melanonota (Gould) sia quella che il Temminck chiamò col nome di Hemipodius maculosus.

\section{(1028) Turnix saturata, Forbes.}

Turnix saturata, Grant, Ibis, 1889, p. 469. - Tristr., Cat. Coll. B. p. 30 (1889).

Turnix melanonotus, Finsch (nec Gould), Vög. d. Südsee, p. 20 (liova Britannia) (1884).

Secondo il Finsch, la Turnix saturata, Forbes, sarebbe la femmina della $T$. melanonota, Gould, la quale cosa non sembra esatta, giacchè quella viene ammessa come distinta dall'Ogilvie-Grant.

(1) Il Ramsay, Tab. List, p. 19, annovera dubitativamente la Excalfacloria australis, Gould, fra le specie della Nuova Guinea meridionale-orientale. 


\section{OR D O GRALLA TORES}

Sp. 1164 (851 $\left.{ }^{\text {bis }}\right)$ Rallina woodfordi, Grant.

Rallina woodfordi, Grant, Ann. and Mag. Nat. Hist. (6) IV, p. 320 (1889).

Supra nigro-brunnea, alis et cauda brunnescentioribus; subtus nigro-ardcsiaca, mento et gula albicantibus ; remigibus primariis intus basin versus et subalaribus maculis transversis albis notatis; rostro (olivaceo) nigro; pedibus griseis; iride rubra.

Long. tot. $0^{\mathrm{m}}, 355$; al. $0^{\mathrm{m}}, 165$; caud. $0^{\mathrm{m}}, 071$; rostri $0^{\mathrm{m}}, 038$; tarsi $0^{\mathrm{m}}, 060$.

Hab. Ins. Guadalcanar, Ins. Salomonis.

Ho esaminato il tipo di questa specie, molto diversa da ogni altra a me nota, e che non mi sembra neppure affine alla $R$. poeciloptera, Hartl., colla quale l'OgilvieGrant la paragona, arendo le remiganti esternamente unicolori.

(849) Hypotaenidia philippensis (Linn.).

Hypotaenidia australis, Bansay, Pr. Linn. Soc. N. S. W. III, p. 39 (1882) (Isole Salomone). Finsch, Vög. d. Südsee, p. 21 (Nova Britannia) (1884).

\section{(850) Rallina fasciata (RAFru.).}

Rallina fasciaia, Meyer, Sitzb. u. Abh. Gesellsch. Isis, 1884, Abh. I, p. 53 (Batchian!.

(851) Rallina tricolor (G. R. Gro).

Rallina tricolor, Mejer, Zeitschr. f. ges. Orn. 1, p. 294 (1884) (uovo). - Nehrk., Journ. f. Orn. 1885, p. 35 (Waigeu). - Ramsay, Tab. List, p. 21 (1888). - Tristr., Cat. Coll. B. p. 27 (1889).

(852) Eulabeornis castaneiventris, GodLd.

Eulabeornis castaneiventris, Mejer, Zeitschr. f. ges. Orn. I, r. 294, Taf. XVII, f. 3 (uovo) (1884).

(853) Gymnocrex plumbeiventris (G. R. GR.).

Rallus intactus, Ramsay, Pr. Linn. Soc. X. S. W. VII, p. 43 (1882).

Gymnocrex plumbeiventris, Meyer, Zeitschr, f. ges, Orn. I, p. $295(1884)_{0}$ - Sharpe, in Gould's B. Lew Guin. pt. XXIV, pl. 12 (1888).

Rallina plumbeiventris, Tristr., Cat. Coll. B. p. 27 (1889).

Il Ramsay manifesta il dubbio che il tipo del $R$. intactus non sia delle Isole Salomone.

(854) Rallicula rubra, Schleg.

Rallicula rubra, Guillem., P. Z. S. 1885, p. 664, pl. XXXIX (Arfak).

(855) Rallicula leucospila (SALVAD.).

Rallicula leucospila, Heine et Rchnw., Nomencl. Nus. Hein. Orn. p. 319 (1890).

[70] 
Sp. 1165 (855 bis) Rallicula forbesi, SHARPE.

Rallicula rubra, Sharpe (nec Schleg.), Nature, vol. 34, p. 340 (1886).

Rallicula forbesi, Sharpe in Gould's Birds of New Guin. pt. XXIlI, pl. 12 (1887). - Ibis, 1889, p. 583.

Mas. Rufo-castaneus, dorso et alis nigris; uropygio nigricante, rufescente transfasciolato; supracaudalibus rufo-castaneis, nigro transfasciolatis; cauda custanea, fasciis fuscis obsoletis notato; lateribus abdomineque imo fuscis, obsolete rufescente fasciatis; subcaudalibus longis, castaneis, late nigro transfasciatis; subalaribus ct axillaribus nigris, albo transfasciatis; remigibus subtus nigris, maculis, vel fasciis albis, vel fulvescentibus in pogonio interno; rostro et pedibus nigris.

Foem. Paullo minor, et dorso alisque nigris maculis rufescente-ochraceis notatis, diversa.

Long. tot. $0^{\mathrm{m}}, 216$; al. $0^{\mathrm{m}}, 108$; caud. $0^{\mathrm{m}}, 062$; rostri culm. $0^{\mathrm{m}}, 029$; tarsi $0^{\mathrm{m}}, 034$.

Hab. in Papuasia - Nora Guinea meridionali-orientali, in Montibus Owen Stanley dictis (Forbes).

Lo Sharpe ha descritto due esemplari, che egli crede maschio e femmina di una medesima specie, la quale si distingue dalla $R$. rubra, Schleg. pel dorso e per le ali decisamente nere nel maschio e macchiettate di ocraceo nella femmina; dalla $R$. leucospila, Salvad. differisce la $R$. forbesi per avere il maschio quelle macchie longitudinali bianche su ambedue i vessilli delle piume del dorso, delle scapolari e delle cuopritrici superiori delle ali, e la femmina per le macchie tondeggianti delle parti superiori e specialmente del dorso di color rossigno ocraceo.

Ho esaminato $\mathrm{i}$ tipi di questa specie, la cui femmina sembra molto simile a quella della $R$. leucospila.

\section{(858) Ortygometra cinereá (VIEILL.).}

Ortygometra cinerea, Meyer, Ibis, 1890, p. 424 (New Britain).

(859) Amaurornis moluccana (WaLL.).

Amaurornis moluccana, Blas. u. Nehrk., Verh. 7.-b. Ges. Wien, 1882, p. 431 (Amboina). Ramsay, Pr. Linn. Soc. N. S, W. VII, p. 665 (St. Anna, Isole Salomone, Mardonald) (1882). Meyer, Sitzb. u. Abb. Gesellsch. Isis, 1884, Abh. I, p. 55 (Siao). - Pleske, Bull. Acad. Petersb. XXIX, p. 536 (Ternate) (1884). - Nortb, Pr. Linn. Soc. N. S. W. (2) II, p. 446 (eggs, New Britain) (1887).

Amaurornis olivacea, Finsch (nec Meyer), Vög. d. Südsee, p. 21 (Nova Britannia) (1884).

Gallinula ruficrissa, North, Pr. Linn. Soc. N. S. W. (2) II, p. 446 (1887)。 - Ramsay, Tab. List, p. 21 , e nota 647 (1888).

(861) Gallinula frontata, WALL.

Gallinula tenebrosa, part., Ramsay, Tab. List, p. 21 (1888?.

\section{(862) Porphyrio melanopterus, TeMm.}

Porphyrio melanotus, Finsch, Vög. d. Siidsee, p. 29 (Port Moresby) (1884).

Porphyrio melanopterus, Forbes, P. Z. S. 1884, pp. 426, 434. - Grant, P. Z. S. 1887, p. 333 (Fauro).

Hab: in Ins. Salomonis, Fauro (Woodford). 
(864) Glareola orientalis, Leach.

Glareola orientalis, Seebh., Geogr. Distr. Charadr. p. 258 (1887).

\section{(865) Stiltia isabella (VIEILL.).}

Glareola isabella, Meyer, Verh. z.-h. Ges, 1881, Wien, p. 769 (Timor). Glareola grallaria, Seebh., Geogr. Distr. Charadr p. 263 (1887).

(866) Haematopus longirostris, VIEILL.

Haematopus longirostris, Seebh., Geogr. Distr. Charadr. p. 304 (1887 - Ramsay, Tab. List, p. 19 (1888).

(867) Strepsilas interpres, LinN.

Strepsilas interpres, Meyer, Vehr. z.-h. Ges. Wien, 1881, p. 767 (Sumba). - Seebh., Geogr. Distr. Charadr. p. 411 (1887). - Ramsay, Tab. List, p. 20 (1888).

(868) Orthorhamphus magnirostris (GEOFR.).

Aesacus (sic) magnirostris, Ramsay, Pr. Linn. Soc. N. S. W. VII, p. 40 (1882) (Isole Salomone). Orthorhamphus magnirostris, Sclat., P. Z. S. 1883, pp. 52, 195, 200 (Kirimoen, Larat, Timorlaut). - Forb., P. Z. S. 1884, p. 433. - Nehrk., Journ. f. Orn. 1885, p. 35 (Waigeu). - Guillem., P. Z. S. 1885, p. 664 (Mysol.). - Meyer, Zeitschr. f. ges. Orn. 1886, p. 38 (Jobi).

Oedicnemus magnirostris, Seebb., Geogr. Distr. Charadr. p. 89 (1887).

Esacus magnirostris, Grant, P. Z. S. 1888, p. 201 (Aola, Guadalcanar). - Ramsay, Tab. List, p. 19 (1888). - De Vis, Ann. Rep. Brit. New Guin. p. 62 (1890). - Id., Golon. Papers, no. 103, p. $116(1890)$. - Id., Ibis, 1891, p. 41.

Hab. in Jobi (Meyer); insula Ferguson (fide De $\mathrm{V} i s$ ).

Gen. OEDICNEMUS, Tenm.

Corrira, Briss., Ornith. VI, p. 542 (1760).

Typus :

Burhinus, Ill., Prodr. Mamm. et Av. p. 250 (1811)

Oedicnemus, Temm., Man. d'Orn. p. 322 (1815).

Fedoa, Leach, Syst. Cat. p. 28 (1816)

Charadrius magnirostris, Lath. Ch. oedicnemus, Linn. Ch. oedicnemus, Linn.

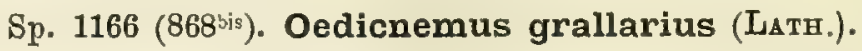

Charadrius grallarius, Lath., Ind. Orn., Suppl. p. I.XVI (1802).

?Charadrius fraenatus, Lath., Ind. Orn. Suppl, p. LXVII (1801). - Id., Gen. Syn., Suppl. p. 320, n. 12 (1802). - Vieill., N. D. XXVII, p. 135 (1818). - Id. Enc. Méth. I p. 334 (1823).

?Charadrius magnirostris, Lath., Ind. Orn. Suppl. p. LXVI, n. 2 (ex Icon, ined. Lamb. II, t. 15, fide G. R. Gray). - Wagl., Syst. Av. gen. Charadrius, Observ, n. 1 (1827.

Burhinus magnirostris, Illig., Pr. Mamm. et Av. p. 250 (1811) (1).

Oedicnemus longipes, Geoffr. in Mus. Paris. - Vieill., N. D. XXIII p. 232 (1818). - Id., Enc. Méth. I, p. 339, pl. 234, f. 2 (1823! - Id., Gal. 0is. II, p. 84, pl. 228 (1825). - Steph., Gen. Zool. XIV, p. 344 (1826). - Temm., Pl. Col. 386 (Livr. 65, 1826), - Less., Tr. d'Orn. p. 546 (1831). - Id., Compl. de Buff. II, p. 617 (1837). - Rchb., Grallatores, tab. CIV, f. 665 (1848). - Schleg., Handl. Dierk. I, p. 439 (1857).

(1) Il Gray (Gen. B. III, p. 535, nota *) afferma che il tipo del genere Burhinus, Illig. è it Charadrius magnirostris, Latham, fondato sulla cattiva figura della tavola 19 delle Icon. ined. del Lambert. 
Oedicnemus magnirostris, Steph., Gen. Zool. XI, 2, p. 462 (1819).

Himantopus grallarius, Steph., Gen. Zool. XII, 1, p. 184 (1824).

Burhinus Novae Hollandiae, Steph., Gen. Zool. XIV, p. 342 (1826).

Charadrius longipes, Wagl., Syst. Av. gen. Charadrius, n. 4 (1827).

Charadrius giganteus (Licht.), Wagl., Isis, 1829, p. 647.

Oedicnemus giganteus, Licht., in Mus. Berol. fide Wagl., Isis, 1829, p. 248, - G. R. Gr., Gen. B. III, p. 535 , n. 6 (1844).

Oedicnemus grallarius, G. H. Gr., List B. Brit. Mus. III, p. 535 (1844). - Id., Gen. B. III, p. 535, n. 5 (1844). - Strickl., Ann. and Mag. N. H. XI, p. 337 (18--?). - Gould, B. Austr. VI, pl. 5 (Part XXI, 1845). - Licht., Nomencl. Av. p. 93 (1854). - Schleg., Handl. Dierk, p. 440 (1857). - Sclat., P. Z. S. 1862, p. 322. - Gould, Handb. B. Austr. II, p. 210 (1865). - Schleg., Mus. P. B. Cursores, p. 18 (1865). - Hamsay, Ibis, 1866, p. 334. - G. R. Gr., Hand-List, III, p. 10, p. 9947 (1871). - Schleg., Dierent. p. 256 (1872) - Garrod, P. Z. S. 1873, p. 469; 1874, p. 112. - Harting, P. Z. S. 1874, p. 459. (Western Australia). - Gieb., Thes. Urn. II, p. 741 (1875). Ramsay, Pr. Linn. Soc. N. S. W. I, p. 59 (1875) (Percy IsL.). - Dress., B. of Eur. VII, p. 407 (1876) - Ramsay, Pr. Linn. Soc. N. S. W. II, p. 196, n. 561 (1877). - Masters, Pr. Linn. Soc. N. S. W. II, p. 275 (1877). - Ramsay, P. Z. S. 1877, p. 335. - Nehrk., Journ. f. Orn. 1879, p. 408. - Salv., Cat. B. Strickl. Coll. p. 590, n. 2883 (1882). - Meyer, Sitzb. u. Abh. Gesellsch. Isis, 1884, p. 215 (Timorlaut). - Nortb, Pr. Linn. Soc. N. S. W. ser. 2a, vol. I, p. 1170 (1886). Seebh., Geogr. Distr. Charadr. p. 83 (1887). - Kamsay, Pr. Linn. Soc. N. S. W. (2) II, p. 171, (1887).

Oedicnemus personatus, Thien., Nidi Av. et ova (Catalogus (184-?).

Burhinus grallarius, Bp., Compt. Rend. XLIII, p. 416, n. 23 (1856).

Oedicnemus australis, Sclat., P. Z. S. 1859, p. 212. - Id., Ibis, 1859, p. 336.

Capistro, superciliis, gula, ventre et subcaudalibus albis; capite supra, occipite, nucha et collo postico griseo-cinereis, lineolis fuscis variegatis; regione parotica, colli lateribus et dorso fuscis; plumis dorsi et scapularibus in medio nigris; uropygio et supracaudalibus griseo-cinereis, in medio macula nigra notatis; collo antico, pectore, epigastrio et tectricibus mediis alarum albido-fulvescentibus, pluma quavis juxta scapum nigra; tectricibus alarum minoribus et majoribus fuscis; remigibus primariis nigris in medio pure albis; rectricibus intermediis griseis irregulariter fusco fasciolatis, lateralibus ad apicem late nigris, in medio albis, basim versus griseis nigro fasciatis et variegatis, extima basim versus albida, nigro fasciata; rostro nigro; pedibus olivaceis, digitis fuscis, iride flava.

Long. tot. circa $0^{\mathrm{m}}, 580$; al. $0^{\mathrm{m}}, 330$; caud. $0^{\mathrm{m}}, 215$; rostri $0^{\mathrm{m}}, 047$; tarsi $0^{\mathrm{m}}, 125$.

Hab. in Nova Hollandia (Latham, Gould, Ramsay); Ins. Percy (Ramsay); in Papuasia - Ins. Timorlaut (Riedel).

Questa specie di Occhione si riconosce facilmente alle sue grandi dimensioni, essendo la maggiore di tutte; pare che essa presenti due varietà, ed anzi il Gould dubitò che gli esemplari delle coste settentrionali della Nuova Olanda potessero costituire una specie distinta; anche lo Schlegel distingue gl' individui de forte taille da quelli de taille faible; il Seebohm non ammette varieta costanti e forse si tratta di variazioni individuali.

L'Oe. grallarius, secondo il Seebohm, sarebbe proprio della parte orientale della Nuova Olanda, ma egli ignorava che questo uccello fosse stato trovato in Timorlaut, la quale cosa ci può far supporre che esso si trovi anche nella parte occidentale della Nuova Olanda; l'Harting ( $l$. c.) lo dice anzi dell'A ustralia occidentale, ma senza dubbio quello fu un lapsus calami. 
L'Oe. grallarius depone due sole uova per covata, sul nudo terreno; esse sono generalmente di color isabellino, macchiate di bruno.

(869) Squatarola helvetica (LINN.).

Charadrius helveticus, Seebh., Geogr. Distr. Charadr. p. 102 (1887). - Ramsay, Tab. List, p. 19 (1888).

\section{(870) Charadrius fulvus, GM.}

Charadrius fulvus, Meyer, Verh. z.-b. Ges. Wien, 1881, p. 767 (Sumba). - Sclat., P. Z. S. 1883, pp. 52, 200 (Maroe, Timorlaut). - Forb., P. Z. S. 1884, p. 433. - Pleske, Bull. Acad. Petersb. XXIX, p. 536 (Ternate) (1884). - Nehrk., Journ. f. Orn. 1885, p. 53 (Waigeu) --Guillem. P. Z. S. 1885, p. 664 (Waigiou). - Seebh., Geogr. Distr. Charadr. p. 99 (1887). - Ramsay, Tab. List, p. 19 (1888). - Tristr., Ibis, 1889, p. 558 - Salvad., Ann. Mus. Giv. Gen. (2) X, p. 827 (1891) (Isola Killerton).

Hab. in Ins. Rossel dicta (Thomson).

(871) Aegialitis vereda (GouLD).

Charadrius veredus, Seebh., Geogr. Distr. Charadr. p. 115 (1887).

(872) Aegialitis geoffroyi (W $\Delta$ GL. ).

Aegialitis geoffroyi, Sclat., P. Z. S. 1883, pp. 52, 200 (Maroe, Timorlaut). - Meyer, Sitzb. u. Abh. Gesellsch. Isis, 1884, Abh. I, p. 55 (Buru, Riedel). - Forb., P. Z. S. 1884, p. 433. - Nehrk., Journ. f. Orn. 1885 , p. 35 (Waigeu). - Guillem., P. Z. S. 1885, p. 664 (Waigiou).

Charadrius geoffroyi, Seebh, Geogr. Distr. Charadr. p. 146.

\section{(873) Aegialitis mongolica (PaLl.).}

Aegialitis mongolica, Nehrk., Journ. f. Orn. 1885; p. 35 (Waigeu). - Guillem.. P. Z. S. 1885, p. 664 (Batanta). - Ramsay, Tab. List, p. 19 (1888).

Charadrius mongolícus, Seebh., Geogr. Distr. Charadr. p. 147 (1887).

Aegialitis mastersi, Ramsay, Tab. List, p. 19 (1888).

\section{(874) Aegialitis ruficapilla (Tемм.).}

Charadrius ruficapillus, Seebh., Geogr. Distr. Charadr. p. 164 (1887). Aegialitis ruficapilla, Ramsay, Tab. List, p. 19 (1888).

Il Seebohm non indica la Nuova Guinea meridionale-orientale fra i luoghi abitati da questa specie e forse l'asserzione del Ramsay rispetto a quella località non è esatta, nel quale caso questa specie dovrà essere esclusa dal novero delle papuane.

(875) Aegialitis jerdoni, LegGe.

Aegialites minor, Finsch, Vög. d. Südsee, p. 5 (Nova Britannia) (1884).

Charadrius fluviatilis, Finsch, ibid. p. 22 (Nova Britannia) (1884).

Charadrius minor, part., Seebh., Geogr. Distr. Charadr. p. 130 (1887).

Charadrius minor jerdoni, Seebh, op. cit, p. 132 (1887).

Haegialitis hiaticula, Ramsay (nec Linn. ?), Tab. List, p. 19 (1888).

Aegialitis jerdoni, Ramsay, op. cit. nota 591.

Il Seebohm riferisce, secondo me, erroneamente all'Ae. minor gli esemplari che si trovano nella Nuova Guinea e nelle isole vicine (1).

(1) Il Ramsay (Tab. List, p. 19) torna ad indicare la Nuova Guinea meridionale-orientale fra i luoghi abitati dalla Aegialilis bicincla (J. et S.), ma senza darci le prove di quest'asserzione ; tuttavia è possibile che la cosa sia esatta. 
(876) Loblvanellus miles (BoDd.).

Lobivanellus miles, Sclat., P. Z. S. 1883, pp. 52, 200 (Larat, Timorlaut). - Forb., P. Z. S. 1884, p. 433.

Lobivanellus personatus, Seebh., Geogr. Distr. Charadr. p. 189 (1887).

(877) Hydralector gallinaceus (TEMM.).

Hydralector gallinaceus, Meyer, Zeitschr. f. ges. Orn. 1886, p. 38 (Baia Walkenaer, sulla costa settentrionale della Nuova Guinea circa al $140^{\circ}$ 1. o.).

Parra gallinacea (part 8), Ramsay, Tab. List, p. 21 (1888).

(878) Himantopus leucocephalus, GodLd.

Himantopus leucocephalus, Seebh., Geogr. Distr. Charadr. p. 283 (1887). - Ramsay, Tab. List, p. 20 (1888). - Tristr., Jbis, 1889, p. 558.

Hab. in Ins. Fergusson dicta (Thomson).

\section{(879) Lobipes hyperboreus (LINN.).}

Phalaropus hyperboreus, Finsch, Vog. d. Südsee, pp. 5, 22 (Nova Britannia) (1884). - Seelbh. Geogr. Distr. Charadr. p. 340 (1887).

(880) Tringa crassirostris, T. et $\mathbf{S}$.

Tringa crassirostris, Meyer, Sitzb. u. Abh. Gesellsch. Isis, 1884, Abh. I, p. 55 (Buru, Riedel). Seebh., Geogr. Distr. Charadr. p. 421 (1887). - Ramisay, Tab. List, p. 20 (1888).

\section{(881) Tringa acuminata (HoRsF.).}

Tringa acuminata, Finsch, Vög. d. Südsee, pp. 5, 22 (Nova Britannia), 39 (Somerset, Torres Str.) (1884). - Guillem., P. Z. S. 1885, p. 664 (Waigiou). - Seebh., Geogr. Distr. Charadr. p. 441 (1887).

Tringa (Limnocinclus) acuminata, Ramsay, Tab. List, p. 20 (1888).

(882) Tringa albescens, Trun.

Tringa minuta, Finsch (nec Auct.), Vög. d. Südsee, pp. 5, 23 (Nova Britannia (1884).

Tringa albescens, Meyer, Sitzb. u. Abb. Gesellsch. Isis, 1884, Abh. I, p. 55 (Sangi). - Guillem., P. Z. S. 1885, p. 576 (Weeda IsI.).

Tringa minuta ruficollis, Seebh., Geogr. Distr. Charadr. p. 437, pl. XV (1887).

Tringa (Schoeniclus) albescens, Ramsay, Tab. List, p. 20 (1888) (1).

\section{(888) Tringoides hypoleucos (LINN.).}

Actitis hypoleucos, Ramsay, Pr. Linn. Soc. N. S. W. VII, p. 41 (1882) (Isole Salomone). - Id., Tab. List, p. 20 (1888).

Tringoides hypoleucos, Blas. u. Nehrk., Verh. z.-b. Ges. Wien, 1882, p. 430 (Amboina). - Meyer, Sitzb. u. Abh. Geselksch. Isis, 1884, Abh. I, p. 55 (Siao, Sangi, Meyer, Buru, Riedel). - Id., Zeitschr. f. ges. Orn.I, p. 295 (1884). - Pleske, Bull. Acad. Petersb. XXIX, p. 536 (Ternate) (1884). - Nehrk., Journ. f. Orn. 1885, p. 35 (Waigeu). - Guillem., P. Z. S. 1885, p. 664 (Waigiou, Mysol). - De Vis, Ann. Rep. Brit. New Guin. p. 62 (1890). - Id., Colon. Papers, no. 103, p. 116 (1890). - Id., Ibis, 1891, p. 41. - Salvad., Ann. Mus. Giv. Gen. (2) X, p. 827 (1891) (Isola Killerton).

Actitis hypoleucus, Finsch, Vög. d. Südsee, p. 22 (Nova Britannia) (1884).

Totanus hypoleucus, Seebh., Geogr. Distr. Gharadr. p. 371 (1887).

Hab. Insula Sud-est dicta (fide $D e$ Vis).

(1) Il Ramsay (Tab. List, p. 20) torna ad indicare la Nuova Guinea meridionale-orientale fra i luoghi (abitati dalla Tringa subarquala (Güld.), ma finora non ci ha dato la prova di questa sua asserzione. 


\section{Gen. Heteractitis, Stejn.}

Heteroscelus, Baird, B. North Am. p. 734 (1858) (nec Heteroscelis, Lath. 1825)

Heteractitis, Stejn., Auk, 1 July 1884, p. 236

\section{(884) Heteractitis brevipes (VIEILI.).}

Totanus incanus, part., Salvad., Orn. Pap. e Mol. III, p. 320 (1882). - Sclat., P. Z. S 1883, pp. 52, 200 (Meloe, Timorlaut). - Meyer, Sitzb. u. Abh. Gesellsch. Isis, 1884, Abh. I, p. 55 (Siao, Sangi). - Forb., P. Z. S. 1884, p. 433. - Guillem., P. Z. S. 1885, p. 665 (Waigiou). - Ramsay, Tab. List, p. 20 (1888).

Totanus brevipes, Ramsay, Pr. Linn. Soc. N. S. W. VII, p. 41 (1882) (St. Cristoval).

Heteractitis brevipes, Stejn., Res. Ornith. Expl. Kamsch. p. 137 (1883). - Gigl. et Salvad., P. Z. S. 1887, p. 586. - Salvad, e Gigl, Mem. R. Ac. Sc. Tor. ser. II, t. XXXIX, p. 115 (1888). Salvad., Ann. Mus. Civ. Gen. (2) X, p. 827 (1891) (Isola Killerton).

Actitis incanus, Finsch (nec Gm.), Vög. d. Südsee, p. 22 (Nova Britannia) (1884)

Totanus incanus brevipes, Seebh., Geogr. Distr. Charadr. p. 361 (1887).

Il Ramsay annovera fra le specie delle Isole Salomone il Totanus brevipes, ed è probabile che gli esemplari che egli attribuisce al medesimo appartengano alla forma occidentale e non alla forma orientale, le quali sono state distinte recentemente dallo Stejneger; questi ha mostrato che la forma dell'Asia Orientale e delle isole vicine, delle isole della Sonda, delle Molucche, della Papuasia e dell'Australia è diversa da quella della costa occidentale dell'America settentrionale e delle isole orientali della Polinesia, alla quale deve essere serbato il nome $H$. incanus (Gm.); in questa il solco nasale sui lati del becco è lungo circa due terzi del culmine, le dimensioni sono un po' maggiori e nell'abito di nozze le parti inferiori, compresi il mezzo dell'addome ed il sottocoda, hanno tutte fascie ondulate grigie scure, ed il dorso di color grigio più puro.

Invece nella $H$. brevipes il solco nasale è lungo soltanto la metà del culmine del becco, le dimensioni sono minori e nell'abito di nozze le fascie ondulate grigie scure delle parti inferiori non si estenclono sul mezzo dell'addome e sul sottocoda, che sono bianchi, le fascie sono più sottili e più chiare ed il dorso è di color grigio più terreo. Inoltre il Seebohm aggiunge un'altra differenza: nella $H$. brevipes il tarso sarebbe posteriormente scudettato, laddove nella $H$. incanus sarebbe reticolato.

(885) Totanus glareola (Link.).

Totanus glareola, Salvad., Voy. Chall., Birds, p. 61 (1881). - Guillem., P. Z. S. 1885 , p. 665 (Waigiou). - Seebh., Geogr. Distr. Charadr: p. 365 (1887).

Hab. in Waigiou (Guillemard).

(886) Totanus stagnatilis, BrCHST.

Totanus stagnatilis, Seebh., Geogr. Distr. Charadr. p. 357 (1887). - Ramsay, Tab. List, p. 20 (1888).

(837) Totanus glottis (LinN.).

Totanus glottis, Seebh., Geogr. Distr. Charadr. p. 355 (1887).

Il nome Linneano deve essere restituito a questa specie. 


\section{(888) Terekia cinerea (Güldenst.).}

Terekia cinerea, Meyer, Sitzb. u. Abb. Gesellsch. Isis, 1884, Abh. I, p. 55 (Ceram Riedel). Totanus terekius, Seebb, Geogr. Distr. Charadr. p. 369 (1887).

(889) Limosa brevipes, G. R. GR.

Limosa melanuroides, Finsch, Vög. d. Südsee, pp. 5, 22 (Nova Britannia) (1884).

Limosa melanura melanuroides, Seebh., Geogr. Distr. Charadr. p. 391 (1887).

Il Seebohm afferma che il nome $L$. brevipes non fu dato a questa specie dal Gray, come si è generalmente ammesso, ma alla L. uropygialis.

(890) Limosa baueri, NADM.

Limosa baueri, Ramsay, Pr. Linn. Soc. N. S. W. VII, p. 41 (1882).

Limosa uropygialis, Finsch, Vög. d. Südsee, pp. 5, 22 (Nova Britannia) (1884).

Limosa rufa uropygialis, Seebh., Geogr. Distr. Charadr. p. 387 (1887).

\section{Sp. 1167 (890 $\left.{ }^{\text {bis }}\right)$ Numenius lineatus, Cuv.}

Numenius arquata, part., Pall., Zoogr. Rosso-As. II, p. 168 (1811).

Courlis à taches étroites de l'Inde, Cuv., Règn. An. I, p. 521 (1829).

Numenius lineatus, Cuv , Règn. An. 1, p. 521 (1829) (Inde). - Liess., Tr. d’0rn. I, p. 565 (1831). - Bp., Compt. Rend. XLIII, p. 597, n. 274 (1856). - Blyth, Ibis, 1867, p. 167. - Swinh., P. Z. S. 1871, p. 410. - Dyb., Journ. f. Orn. 1873, p. 103. - Hume, Str. Feath. 1, p. 237 (1873). Adam, Str. Feath. I, p. 396 (1873). - Hume, Str. Feath. II, p. 296 (1874). - Tacz., Journ. f. Orn. 1874, p. 336. - Blyth, B. of Burma, p. 155 (1875). - Butler, Str. Feath. IV, p. 16 (1876). - Armstrong, Str. Feath. IV, p. 341 (1876). - Hume, Str. Featb. IV, p. 464 (1876). - Tacz., Bull. Soc. Zool. de France, I, p. 255 (1876). - David et Oust., Ois. Chine, p. 57 (1877). - Hume et Davis. Str. Feath. VI, p. 460 (1878). - David et Wander, Str, Feath. VII, p. 89 (1878). - Hume Str. Feath. VIII, p. 112, n. 877 (1879). - Scully, Str. Feath. VIII, p. 356 (1879). - Legge, B. of Ceyl. p. 306 (1880). - Oat., Str. Feath. X, p. 239 (1882). - Seebh., Ibis, 1884, pp. 34, 268. - Blak., Amend. List B. of Jap. p. 39 (1884). - Swinh. and Barnes, Ibis, 1885, p. 134. - Sharpe, Ibis, 1888 , p. 202. - Blas., Ibis, 1888, p. 373.

Numenius arquata, Sundev., (nec Linn.), Phys. Sälsk. Tidskr. 1838, p. 203. - Blyth, Cat. B. Mus. A. S. B. p. 268, o. 1590 (1849). - Kelaart, Prodr. Cat. p. 134 (1852). - Layard, Ann. and Mag. N. H. XIV, p. 264 (1854). - Schleg., Mus. P. B. Scolopaces, p. 85 (partim) (1864). - Layard, B. of S. Afr. p. 322 (1867). - G. R. Gr., Hand-List, III, p. 42, n. 10239 (partim)(1871). - Holdsw., P. Z. S. 1872 , p. 474. - Legge, Ibis, 1874, p. 29. - Salvad., Cat. Ucc. Born. p. 332 (1874). Legge, Ibis, 1875, p. 402.

Númenius nasicus, Temm., Man. d'Orn. IV, p. 393 (1840) (Grand Archipel Asiatique). - Tacz., Journ. f. Orn. 1871, p. 58.

Numenius arquatula, Hodgs, Gray's Zool. Miscell. I, p. 86 (1844). - Gray, Cat. Hodgs. Coll. p. 137 (1846).

Numenius major, part., Temm, et Schleg. (nec Steph.), Faun. Jap. Aves, p. 110 (?) (nec Tab. LXVI) (1847). - Swinh., Ibis, 1860, p. 66; 1863, p. 410. - Id., P. Z. S. 1863, p. 318. - Schleg., Mus. P. B. Scolopaces, p. 89 (1865). - Whitely, Ibis, 1867, p. 205. - Swinh., lbis, 1867, p.391; 1870, p. 303. - Salvad., Cat. Ucc. Born. p. 333 (1874). - Swinh., Ibis, 1876, p. 334. - Blakist. and Pryer, lbis, 1878, p. 222. - Id., Tr. As. Soc. of Jap. X, p. 115 (1882). - Vorderm., Nat. Tijdshr. Ned. Ind. XLIII, p. 117 (1883). - - S Sharpe, Ibis, 1886, p. 168 (Muscat).

Numenius arquatus, Irby, Ibis, 1861, p. 240. - Swinh., Ibis, 1863, p. $410 ; 1867$, p. 391. - Id., P. Z. S. 1863, p. 318 - Dyb., Journ. f. Orn. 1868, p. 337 (Dauria). - Layard and Sharpe, B. of S. Afr. p. 692 (1884).

Numenius arcuatus, Cass. (nec Linn.), Pr. Ac. Philad, 1862, p. 321. - Gray, Cat. Hodgs. Coll.

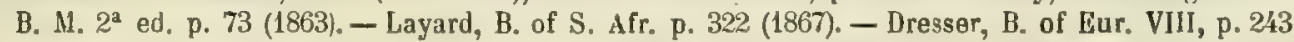
(partim) (1873). - Hume, Str. Feath. III, p. 182 (1875). - Oat., B. of Brit. Burm. Il, p. 412 (1883). - Seebh., Ibis, 1887, p. 182.

Numenius cassini, Swinh., Ibis, 1867, p. 398 (Amoy). - G. R. Gr., Hand-List, III, p. 42, n. 10245 (1871). 
Numenius subarquata, Hume, Str. Feath. II, p. 297 (1874) (Port Blair).

Numenius arquatus lineatus, Seebb., Geogr. Distr. ('haradr. p. 324 (1887).

Numenius arquata lineata, Seebh., Ibis, 1888, p. 236.

Numenius N. arquatae (Linn.) simillimus, sed major, et rostro longiore, uropygio albo fere immaculato, subalaribus albis immaculatis vel lineis scapalibus fuscis notatis, et praesertim plumis laterum eodem modo notatis, diversus.

Hab. in India (Cuvier, Hume) ; Ceylon (Kelaart Layard, Legge); ins. Andaman (Hume); ins. Nicobar (Hume); ins. Lacadivis (Hume); Burma (Blyth, Armstrong, Davison) ; Sina (Sivinhoe David); Formosa (Swinhoc); Hainan (Swinhoe); Sibiria (Dybowschi); Japonia (Siebold, Bïrger); ins. Loo-choo (Kern, fide Cassin) ; ins. Philippinis, Palawan (Whitehead); Borneo (Croockevit); Sumatra (Henrici, S. Müller); Java (Kuhl et $\nabla$. Hasselt, Vorderman); in. Moluccis-Halmahera (Mus. Lugd. fide Schlegel); in Africa meridionali (Mus. Lugd., Layard).

Questa specie è il rappresentante Orientale del $N$. arquata (Linn.), dal quale differisce pei caratteri sopraindicati. Il Legge fa notare che il carattere più cospicuo che distingue questa specie è la forma lineare delle-macchie scure delle piume dei fianchi, laddove quelle macchie si allargano trasversalmente nel $N$. arquata.

La femmina differisce dal maschio per le dimensioni maggiori e quindi anche pel becco più lungo.

L'Harting, secondo quanto afferma l'Oates, vorrebbe che il $N$. lineatus fosse fondato sugli esemplari in abito estivo del $N$. arquata, la quale cosa viene negata dal Seebohm ed anche a me non sembra esatta.

Questa specie dall'Asia orientale-settentrionale, ove nidifica, emigra, e nell'inverno si trova abbondante nell'India e nell'arcipelago della Sonda e si estende fino nell'Africa meridionale.

Nelle Molucche trovasi anche, ma forse vi è rara, giacchè non si conosce altro esemplare che quello di Kao, in Halmahera, conservato nel Museo di Leida.

Questa specie è la seconda che sia stata per inavvertenza ommessa nella mia Ornitologia, che ne comprende oltre a mille.

(891) Numenius cyanopus, Vieill.

Numenius australis, Meyer, Verh. z.-b. Ges. Wien, 1881, p. 767 (Sumba).

Numenius cyanopus, Seebh., Geogr. Distr. Charadr. p. 326 (1887). - Ramsay, Tab. List, p. 20 (1888).

\section{(892) Numenius variegatus (Scor.).}

Numenius uropygialis, Meyer, Verb. z.-b..Ges. Wien, 1881, p. 767 (Sumba). - Finsch, Vög. 1 . Sïdsee, n. 22 (Nova Britannia), 39 (1884). - Meyer, Sitzb. u. Abh. Gesellsch. Isis, 1884, Abh. I, p. 56 (Siao), - Pleske, Bull. Acad. Petersb. XXIX, p. 537 (Ternate) (1884). - Guillem., P. Z. S. 1885, p. 576 (Obi Major). - Ramsay, Tab. List, p. 20 (1888).

Numenius variegatus, Sclat, P. Z. S. 1883, pp. 52, 200 (Larat, Timorlaut). - Forb., P. Z. S. 1884, p. 433. - Nehrk., Journ. f. Orn. 1885, p. 35 (Waigeu). - Grant, P. Z. S. 1888, p. 201 (Aola, Guadalcanar).

Numenius phaeopus variegatus, Seebh., Geogr. Distr. Charadr. p. 330 (1887).

Hab. in Ins. Salomonis (Woodford) ; Nova Britannia (Finsch); Timorlaut (Forbes); Obi majore (Guillemard). 
(898) Numenius minutus, Govid.

Numenius minutus, Neyer, Zeitschr. f. ges. Orn. I, p. 295 (Aru, Ribbe) (1884)。 - Seebb., Geogr. Distr. Charadr, p. $335(1887)$.

\section{(894) Scolopax rosenbergi, Schleg.}

Scolopax rosenbergi, Guillem., P. Z. S. 1885, p. 665 (Arfak) - Seebh., Ibis, 1886, p. 128. Salvad., Ibis, 1889, pp. 108-11\%.

Scolopax saturata, part., Seebb., Ibis, 1887, p. 283. - Id., Geogr. Distr. Charadr. p. 506 (partim) (1882).

Contro l'opinione del Seebohm, il quale vorrebbe che questa specie fosse identica colla S. saturata, Horsf. di Giava, io ho dimostrato che essa è affatto distinta pel colorito più oscuro, arendo gli spazi neri fra le fascie castagne molto più larghi ; inoltre essa differisce per avere le fascie scure sulla parte inferiore del collo molto più arcuate, jer la quale cosa appare un disegno a squame, laddove nella specie di Giava le fascie sono più diritte, e più regolarmente trasversali.

Si può aggiungere che nella specie della Nuova Guinea la linea nera dall'occhio alla base del becco è più larga, le fascie chiare delle parti inferiori sono piủ biancheggianti, il petto lungo il mezzo è più biancheggiante, le remiganti primarie sono più scure ed hanno poche macchie rossigne verso l'apice del vessillo esterno, mentre nella specie di Giava tutte le remiganti sono più macchiettate di rossigno sul vessillo esterno, le cuopritrici delle remiganti primarie sono meno macchiettate di rossigno, le cuopritrici inferiori delle ali sono più nereggianti e meno fasciate, e la coda più oscura.

(895) Scolopax rochusseni, SCHLEG.

Scolopax rochusseni, Seebh., Geogr. Distr. Gharadr. p. 505, pl. XX (1887).

(896) Gallinago megala, SWINH.

Gallinago megala, Swinh., Geogr. Distr. Charadr. p. 479 (1887).

(897) Ardea sumatrana, RAFFL.

Ardea sumatrana, Sclat., P. Z. S. 1883, pp. 52, 200 (Larat, Timorlaut). - Meyer, Sitzb. u. Abh. Gesellsch. Isis, 1884, Abh. I, p. 56 (Siao Meyer, Buru Ricdel). - Forb., P. Z. S. 1884, p. 434. Nehrk, Journ. f. Orn. 1885, p. 35 (Waigeu).

(898) Ardea novae hollandiae, Lath.

Demiegretta Novae Hollandiae, Meyer, Verh. z.-b. Ges. Wien, 1881, p. 767 (Sumba).

Ardea Novae Hollandiae, Meyer, Zeitschr. f. ges. Orn. I, pp. 192, 216 (1884) (Timorlaut Riedel).

$$
\text { (899, 900) Ardea picata, Gould. }
$$

Ardea picata, Büttik, Not. Leyd. Mus. VIII, p. 67 (Is. Tenimber) (1886).

Nuova per le isole Tenimber.

Anche il Büttikofer crede, come io areva sospettato, che l'A. aruensis, G. R. Gr. sia fondata sopra un giovane dell' $A$. picata.

(901) Demiegretta sacra (Grr.).

Ardea sacra, Sclat., Voy. Chall. II, pt. VIII, p. 31 (1881). - Meyer, Verh. z.-b. Ges. Wien, 1881, 
p. 767 (Sumba). - Ramsay, Pr. Linn. Soc. N. S. W. VII, p. 42 1882) (St. Cristoval). - Finsch, Vög. d. Südsee, p. 39 (Somerset, Ins. Torres Str.) (1884).

Demiegretta sacra, Sclat., P. Z. S. 1883, pp. 52, 195, 200 (Larat, Kirimoen, Timorlaut) - Meyer, Sitzb. u. Abh. Gesellsch. Isis, 1884, Abh. I, p. 56 (Siao Meyer, Ceram Riedel). - Forb., P. Z. S. 1884, p. 434. - Pleske, Bull. Acad. Petersb. XXIX, p. 537 (Ternate) (1884). - Nehrk., Journ. f. Orn. 1885, p. 35 (Waigeu). - Guillem., P. Z. S. 1885, p. 665 (Salwatti). - Grant, P. Z. S. 1887, p. 333 (Fauro); 1888, p. 202 (Rubiana, Is. Salomone). - Ramsay, Tab. List, p. 21 (1888;

(902) Herodias torra (BסCH. HAM.).

Herodias torra, Meyer, Sitzb. u. Abh. Gesellsch. Isis, 1884, Abh. I, p. 56 (Timorlaut, Ceram Riedel). - Pleske, Bull. Acad. Petersb. XXIX, p. 537 (Ternate) (1884).

Herodias alba, Forb. (nec Linn.), P. Z. S. 1884, p. 434.

\section{(903) Herodias intermedia (HASs.).}

Herodias intermedia, Meyer, Verh. z.-b. Ges. Wien, 1881, p. 767 (Sumba). - Id., Sitzb. u. Abh Gesellsch. Isis, 1884, p. 56 (Celebes). - Ramsay, Tab. List, p. 21 (1888).

? Herodias melanopus, Ramsay, I. c.

(904) Herodias garzetta (LinN.).

Herodias nigripes, Meyer, Verh. za-b. Ges. Wien, 1881, p. 767 (Sumba).

Herodias garzetta, Pleske, Bull. Acad. Petersb. XXIX, p. 537 (Ternate) (1884). - Ramsay, Tab. List, p. 21 (1888).

(905) Herodias immaculata, Godud.

Herodias immaculata, Meyer, Sitzb. u. Abh. Gesellsch. Isis, 1884, Abh. I, p. 56 (Geram, Aru Riedel).

(906) Bubulcus coromandus (Bodd.).

Bubulcus coromandus, Meyer, Sitzh. u. Abh. Gesellsch. Isis, 1884, Abh. I, p. 57(Sangi). - Pleske, Bull. Acad. Petersb. XXIX, p. 537 (Ternate) (1884).

\section{(907) Butorides javanica (HorsF.).}

Butorides javanica, Salvad., Voy. Chall., Birds, p. 64 (1881). - Meyer, Verh. z.-b. Ges. Wien, 1881, p. 767 (Sumba). - Blas., P. Z. S. 1882, p. 710 (Ceram). - Meyer, Zeitschr. f. ges. Orn. I, p. 295 (uova) (1884). - Pleske, Bull. Acad. Petersb. XXIX, p. 538 (Ternate) (1884). - Nehrk., Journ. f. Orn. 1885, p. 35 (Waigeu). - Grant, P. Z. S. 1887, p. 333 (Fauro); 1888, p. 202 (Aola, Guadalcanar). - Salvad, Ann. Mus. Giv, Gen. (2) X, p. 827 (1891) (Kapa-Kapa).

Butoroides javanica, Ramsay, Pr. Linn. Soc. N. S. W. VII, p. 40 (1882) (Isole Salomone). - Id., Tab. List, p. 21 (1888).

Butoroides stagnatilis, Ramsay, ibid. (Isole Salomone).

Il Ramsay, fra gli uccelli delle Isole Salomone, annovera come specie distinte la $B$. javanica e la $B$. stagnatilis, che vengono generalmente considerate come identiche.

(908) Ardetta sinensis (GM.).

Ardetta sinensis, Finsch, Vög. d. Südsee, p. 21 (Nova Britannia) (1884): - Guillem., P. Z. S. 1885 , p. 665 (Andai).

\section{GeN. ARDEIRALLA, VerR.}

Dupetor, Heine, Nomencl. Mus. Hein. Orn. p. 308 (1890) (=Ardeiralla, Verr.). 
(909) Ardeiralla flavicollis (Latr.).

Ardetta flavicollis, Finsch, Vög. d. Südsee, p. 21 (Nova Britannia) (1884).

Ardeiralla flavicollis, Blas. u. Nehrk., Verh. z.-b. Ges. Wien, 1882, p. 431 (Amboina). - Meyer, Sitzb. u. Abh. Gesellsch. Isis, 1884, Abh. I, p. 57 (Siao).

Butoroides flavicollis, Ramsay, Tab. List, p. 21 (1888).

\section{Sp. 1168 (909bis). Ardeiralla woodfordi, Grant (?).}

Ardeiralla woodfordi, Grant, P. Z. S. 1888, p. 202.

Foemina. Capite nigro-cinereo, sensim in colli, dorsi tectricumque alarum colorem saturäte rufo-castaneum transeunte; uropygio et supracaudalibus fuscis, plumarum marginibus rufo-cinnamomeis; remigibus et rectricibus nigro cinereis, capite concoloribus; gula et collo (antico?) fusco maculatis; gastraeo reliquo rufocinnamomeo, abdomine magis cinnamomeo et minus rufescente; axillaribus fulvescente-albidis, tectricibus alarum inferioribus cinnamomeis et castaneis; remigibus subtus nigro-cinereis; scapularibus diffractis alis longioribus.

Long. tot. $0^{\mathrm{m}}, 467$; al. $0^{\mathrm{m}}, 193$; caud. $0^{\mathrm{m}}, 066$; rostri culm. $0^{\mathrm{m}}, 078$; tarsi $0^{\mathrm{m}}, 068$.

Hab. in Papuasia - Ins. Salomonis, Guadalcanar (Woodford).

Secondo l'Ogilvie-Grant questo airone si allontana da tutte le specie conosciute, tuttaria egli crede che si arvicini alquanto alla Ardeiralla flavicollis.

Il suddetto dice che una femmina quasi adulta differisce dal tipo nei seguenti rispetti: alcune piume del capo hanno margini trasversali chiari, le cuopritrici superiori delle ali sono di color cannella chiaro con macchie subterminali in forma di $\nabla$ scure : le remiganti sono marginate di fulvo e le remiganti secondarie esterne sono rossigne chiare con una macchia subapicale senza margine di fulvo.

Un maschio giovane ha la testa nera cinerea, le parti superiori di color rossigno scuro e nericcio, colle piume marginate di fulvo pallido, la metà basale delle cuopritrici delle ali nero-cineree, ciascuna penna terminando con tre fascie parallele trasverse, una di color rossigno-cannella, un'altra scura ed una terza fulva chiara ; inoltre il groppone ed il sopraccoda sono di color cenerino scuro coi margini delle piume fulvi; le gote e le parti inferiori cinnamomee, volgenti al bianchiccio sudicio posteriormente; sulla gola e sul collo sono macchie scure; le ascellari sono bianchiccie; le cuopritrici inferiori delle ali di color grigio scuro variegato di fulvo e le remiganti nere cineree inferiormente.

Ho veduto i tipi di questa specie, che a me sembra molto somigliante alla femmina dell' $A$. flavirostris, se pure ne è diversa.

\section{(910) Ardeiralla melaena (SALVAD.).}

Ardeiralla melaena, Meyer, Sitzb. u. Abb. Gesellsch. Isis, 1884, p. 57.

(911) Zonerodius heliosylus (Less.).

Zonerodius heliosilus, Salvad., Ann. Mus. Giv. Gen. (2) IX, p. 591 (1890) (Bigo). - Heine u. Rchnw., Nomencl, Mus. Hein. Orn. p. 309 (1882-1890).

6 T. Salvadori. 
(913) Nycticorax caledonicus (GM.).

Nycticorax caledonicus, Meyer, Verh. 2.-b. ges. Wien, 1881, p. 771 (Luang.). - Blas. u. Nehrk., Verh. 7.-b. Ges. Wien, 1882, p. 431 (Amboina): - Meyer, Sitzb. u. Abh. Gesellsch. Isis, 1884, Abb. I, p. 57 (Timorlaut Ricdel). - Forb., P. Z. S. 1884, p. 434. - Pleske, Bull. Acad. Petersb. XXIX, p. 538 (Ternate) (1884). - Nehrk., Jouri. f. Orn. 1885, p. 35 (Waigen). - Guillem., P. Z. S. 1885, p. 665 (Salwatti) - Ramsay, Tah. List, p. 21 (1888). - Salvad., Ann. Mus. Civ. Gen. (2), X, p. 827 (1891) (Isola Killerton).

Sp. 1169 (913 $\left.{ }^{\text {bis }}\right)$ Nycticorax mandibularis, GraNT.

Nycticorax manillensis, part., Bp., Consp. II, p. 140 (juv. ex ins. Salomonis) (1885). - G. R. Gr., List B. Trop. Isl. p. 49 (1859). - Sclat., P. Z. S. 1869, p. 119. - G. R. Gr., Hand-List, III, p. 33, n. 10173 (partim) (1871). - Ramsay, Pr. Linn. Soc. N. S. W. VII, p. 39 (1882).

Nycticorax caledonicus, part, Salvad., Orn. Pap. e Mol. III. p. 372 (1882). - ? Ogilvie-Grant, P. Z. S. 1887 , p. 333 (Fauro, Is. Salomone).

Nycticorax mandibularis, Grant, P. Z. S. 1888, p. 203.

Piteo et crista nigris, plumis elongatis occipitis albis, in apice nigris et juxta apicem rufescentibus; corpore supra, cauda et alis sordide castaneis, uropygio et supracaudalibus laetioribus; fascia superciliari obsoleta alba; genis, collo, pectore et lateribus laete castaneis; gula, abdomine et subcaudalibus albis; axillaribus castaneis, marginibus pallidioribus; remigibus rectricibusque subtus pallide griseorufis; rostro nigro, mandibulae basi et cute nuda circumoculari, pedibus et iride flavis.

Long. tot. $0^{\mathrm{m}}, 470$; al. $0^{\mathrm{m}}, 251$; caud. $0^{\mathrm{m}}, 094$; rostri culm. $0^{\mathrm{m}}, 074$; tarsi $0^{\mathrm{m}}, 84$.

Hab. in Papuasia - Ins. Salomonis (Hombron et Jacquinot), Ugi (Morton), St. Cristoval (Stephen), Guadalcanar (Woodford).

Secondo l'Ogilvie-Grant questa specie occupa un posto intermedio fra il $N$. manillensis, Vig. ed il $N$. caledonicus $(\mathrm{Gm}$.$) , cui è forse più affine che non al$ primo. Essa si distingue facilmente da ambedue per le dimensioni minori e pel becco più piccolo ; somiglia al primo pel colore castagno del petto e del collo, ma ne differisce per la gola bianca ; si distingue poi immediatamente dal secondo per la mancanza della fascia sopraccigliare bianca, tanto cospicua nel $N$. caledonicus. Anche i giovani delle due specie si possono facilmente distinguere, giacchè quello del $N$. mandibularis è generalmente più oscuro ed ha le strie lungo lo stelo, e le macchie sulle piume delle parti superiori fulvo-rossigne, anzichè bianco-giallognole.

L'uovo, inviato al Ramsay e raccolto da Mr. Stephen nell'Isola Ugi, somiglia a quello del $N$. caledonicus, avendo lo stesso colore verde-azzurrognolo pallido.

Il Ramsay afferma di avrer ricevuto questa specie anche dalla Nuova Britannia e dalle Isole del Duca di York.

La storia di questa specie è alquanto imbrogliata. Il Bonaparte menzionò un esemplare delle Isole Salomone esistente nel Museo di Parigi e che sarebbe stato portato dal Dussumier. Io, per notizia avuta dall'Oustalet, feci notare $(l . c$. ) che quell'esemplare non era stato raccolto dal Dussumier, che non è mai andato nelle Isole Salomone, ma da Hombron e Jacquinot, durante il Voyage au Pôle Sud. L'Oustalet, scrivendomi di quell'esemplare, che è un giovane, mi avvertiva come esso differisse dai giovani della Nuova Caledonia per le macchie e pei tarsi più lunghi (?), e perciò non era punto sicuro che appartenesse al $N$. caledonicus (Gm.). Poscia il Ramsay, sebbene riconoscesse che gli esemplari delle Isole Salomone non corrispondevano alla descrizione 
del $N$. manillensis, Vig., tuttavia li riferiva a questa specie! Finalmente l'OgilvieGrant li ha separati specificamente.

Ho esaminato un esemplare nel Museo Britannico ; non è esatto che esso manchi del sopracciglio bianco; questo è presente, ma più stretto e meno cospicuo, che non nel $N$. caledonicus; il becco è realmente più breve.

(914) Xenorhynchus asiaticus (LATH.).

Xenorbynchus asiaticus, Ramsay, Tab. List, p. 20 (1888).

\section{Genus platalea, Linn.}

Platea, Briss., Orn. V, p. 351 (1760).

Platalea, Linn., S. N. I, p. 231 (1766)

Spatherodia, Hch Ay Syst Nat $\mathrm{xvi}(1852) \cdot{ }^{\circ} \cdot$

Leucerodia, Rchb., ibid. (1852)
Typus:

Pl. leucorodia, Linn.

$P l$. melanorhynchos, Bchb.

Pl. tenuirostris, Temm.

Sp. 1170 (914 $\left.{ }^{\text {bis }}\right)$ Platalea intermedia, GraNT.

Platalea intermedia, Grant, Ibis, 1889, p. 52, pl. I, f. 2, 2A. - Ibis, 1889, p. 583.

Platalea P1. melanorhynchae, Rchb. affinis, sed cute nuda frontis et gulae et rostri culmine nigerrimis; macula frontali postica, et altera suboculari, flavis; regione supraoculari macula flava destituta; spatula rostri rotundata, nec obtuse truncata; plumis, remigum primariarum apicibus nigris exceptis, omnino albis; occipite cristato.

Long. al. $0^{\mathrm{m}}, 330$; rostri culm. $0^{\mathrm{m}}, 178-0^{\mathrm{m}}, 162$; tarsi $0^{\mathrm{m}}, 134-0^{\mathrm{m}}, 110$.

Hab. in Papuasia - Nova Guinea, prope Portum Moresby (Romilly) - in Borneo (Hoedt).

L'Ogilvie-Grant ha fondato questa specie sopra una testa ed un piede di un esemplare (maschio) aruto da Mr. Romilly presso Port Moresby nella Nuova Guinea; alla stessa specie l'Ogilvie-Grant riferisce due esemplari conservati nel Museo di Leida ed inviati dall'Hoedt come raccolti in Borneo, ma di questi s'ignora la precisa località; essi sono indicati come femmine; forse nessuno dei tre è al tutto adulto.

L'Ogilvie-Grant, fa notare, oltre ai caratteri sopra indicati, che le piume della fronte non si avanzano fino alla linea che si supponga congiungere $i$ margini posteriori degli occhi ; esse distano $0^{\mathrm{m}}, 025$ dalla base del culmine; il margine della pelle nuda della gola dista $0^{\mathrm{m}}, 036$ dall'angolo della bocca; il detto margine ha leggermente le forme di VV posteriormente.

Il principale carattere pel quale questa specie si distingue sarebbe la forma della spatola rotondata all'apice, mentre quella spatola è ottusamente troncata nella Pl. melanorhyncha.

\section{(915) Ibis molucca, Cur.}

Ibis molucca, Nehrk., Journ.' f. Orn. 1885, p. 35 (Waigeu).

Threskiornis strictipennis, Ramsay, Tab. List, p. 20 (1888).

Suppongo che per errore tipografico nella Tabular List del Ramsay il segno relativo al luogo Nuova Guinea meridionale-orientale sia stato trasportato da questa specie al Geronticus spinicollis, Jameson, che è specie esclusivamente australiana. 


\section{ORDo A N S E R ES}

(918) Nettopus pulchellus, Goord.

Nettapus pulchellus, Sclat., P. Z. S. 1883, pp. 52, 200 (Larat, Timorlaut : - Finsch, Vög. d. Südsee, p. 29 (Port Moresby) (1884). - Forb., P. Z. S. 1884, p. 434. - Ramsay, Tab. List, p. 22 (1888).

\section{(919) Dendrocycna arcuata (Cov.).}

Dendrocygna vagans, Ramsay, Tab. List, p. 22 (1888).

\section{(920) Dendrocycna guttata (Forsten).}

Dendrocygna guttata, Sclat,, P. Z. S. 1883, pp. 52, 200 (Larat, Timorlaut). - Finsch, Vög. d. Südsee, p. 29 (1884). - Forb., P. Z. S. 1884, p. 434.

\section{(921) Tadorna radjah (GARN.).}

Tadorna radjab, Sclat., P. Z. S. 1883, pp. 52, 195, 200 (Larat, Timorlaut). - Finsch, Vög. d. Südsee, p. 29 (1884). - Meyer, Sitzb. u. Abh. Gesellsch. Isis, 1884, Abh. I, p. 57 (Ceram Riedel). - Forb., P. Z. S. 1884, p. 434. - Meyer, Zeitschr. f. ges. Orn. I, p. 295 (1884). - Pleske, Bull. Acad. Petersb. XXIX, p. 538 (Ternate) (1884). - Guillem., P. Z. S. 1885, p. 665 (Waigiou, Jobi). Ramsay, Tab. List, p. 22 (1888). - Tristr., Ibis, 1889, p. 558. - Salvad., Ann. Mus. Civ. Gen. (2) X, p. 828 (1891).

Anas radjah, Büttik., Not. Leyd. Mus. VIII, p. 68 (1886).

Hab. in Jobi (Guillemard); ins. Fergusson (Thomson).

\section{(922) Anas superciliosa (Gir.).}

Anas superciliosa, Aamsay, Pr. Linn. Soc. N. S. W. VII, p. 41 (1882) (Isole Salomone). - Meyer, Sitzb. u. Abh. Gesellsch. Isis, 1884, Abh. I, p. 57 (Celebes'- - Grant, P. Z. S. 1888, p. 187 (Isole Salomone). - Ramsay, Tab. List, p. 22 (1888).

Il Ramsay fa notare che gli esemplari delle Isole Salomone sono molto piu piccoli degli Australiani e ne dà le seguenti dimensioni : Long. tot. $0^{\mathrm{m}}, 457$; al. $0^{\mathrm{m}}, 210$; caud. $0^{\mathrm{m}}, 093$; becco $0^{\mathrm{m}}, 048$; tarso $0^{\mathrm{m}}, 035$. Essi dorranno essere ulteriormente confrontati (1).

\section{(923) Fregata aquila (Linn.).}

Fregata aquila, Pleske, Bull. Acad. Petersb. XXIX, p. 538 (Ternate) (1884). Atagen aquila, Ramsay, Tab. List, p. 25 (1888).

(1) Il Ramsay (Tab. List, p. 22) indica sempre dubitativamente la Nuova Guinea meridionaleoríentale fra i luoghi abitati dall'Anas gibberifrons, S. Mull. 
(924) Fregata minor, Briss.

Fregata minor, Pleske, Bull. Acad. Petersb. XXIX, p. 539 (Ternate) (1884) - Büttik., Not. Leyd. Mus. VIlI, p. 68 (Is. Tenimber) (1886).

Atagen minor, Ramsay, Tab. List, p. 25 (1888).

Nuova per le isole Tenimber.

(925) Plotus novae hollandiae, Godid.

Plotus novae hollandiae, Ramsay, Tab. List, p. 24 (1888).

(927) Microcarbo melanoleucus (VIEILL.).

? Carbo melanüus, Bernst., Dagboek, p. 119 (Sorong) (1883).

Graculus (Melanocarbo) melanoleucus, Bernst., l. c:

Microcarbo melanoleucus, Meyer, Zeitschr. f. ges. Orn. I, p. 295 (1884). - Pleske, Bull. Acad. Petersb. XXIX, p. 539 (Ternate) (1884). - Guillenı, P. Z. S. 1885, p. 665 (Mysol, Waigiou). Salvad., Ann. Mus. Civ, Gen. (2) IX, p. 591 (1890) (Rigo).

Graculus melanoleucus, Ramsay, Tab. List, p. 25 (1888).

(929) Pelecanus conspicillatus, TeMr.

Pelecanus conspicillatus, Ramsay, Tab. List, p. 25 (1888).

(930) Sula cyanops (Sond.).

Sula personata, Ramsay, Pr. Linn. Soc. N. S. W. VII, p. 42 (1882) (Isole Salomone).

(981) Sula piscator (LINN.).

Sula fiber, Ramsay, Pr. Linn. Soc. N. S. W. VII, p. 42 (1882) (Isole Salomone).

Sula piscator, Ramsay, Tab. List, p. 24 (1888).

(982) Sula leucogastra (Bodd.).

Sula fusca, Ramsay, Pr. Linn. Soc. N. S. W. VII, p. 42 (1882) (Isole Salomone).

Sula fiber, Blas, u. Nehrk. (nec Linn.), Verh. z,-b. Ges. Wien, 1882, p. 432 (Amboina).

Sula leucogaster, Finsch, Vög. d. Südsee, p. 22 (Nova Britannia) (1884). - Meyer, Sitzb. u. Abh. Gesellsch. Isis, 1884, Abh. I, p. 57 (Siao, Sangi Heyer, Timor Riedel). - Ramsay, Tab. List, p. 24 (1888). - Salvad., Aun. Mus. Civ. Gen. (2) IX, p. 591 (1890) (Rigo).

\section{(933) Phaeton candidus (Briss.).}

Phaeton flavirostris, Ramsay, Pr. Linn. Soc. N. S. W. VII, p. 42 (1882) (Isole Salomone). Ramsay, Tab. List, p. 23 (1888) (1).

\section{(936) Sterna bergii, Licht.}

Sterna burgeri (sic), Ramsay, Pr. Linn. Soc. N. S. W. VII, p. 41 (1882) (Isole Salomone).

Sterna bergii, Finsch, Vög. d. Südsee, p. 22 (INova Britannia), p. 51 (Ins. Marshall)(1884). - Meyer, Sitzb. u. Abh. Gesellsch. Isis, 1884, Abh. I, p. 57 (Ceram, Buru Riedel). - Pleske, Bull. Acad. Petersb. XXIX, p. 539 (Ternate) (1884). - Nehrk., Journ. f. Orn. 1885, p. 35 (Waigeu) - Ramsay, Tah. List, p. 23 (1888)。 - Salvad., Ann. Mus. Civ. Gen. (2) IX, p. 591 (1890) (Rigo); X, p. 833 (1891) (Isola Goodenough).

(938) Sterna media, HorsF.

Sterna media, Ramsay, Tab. List, p. 23 (1888).

(1) L'asserzione ripetuta dal Ramsay (Tab. Lisl, p. 23) che nella Nuova Guinea meridionale-orientale si trovi anche il Phaton rubricauda, Bodd. abbisogna di prove. 
(939) Sterna longipennis, NordM.

Sterna frontalis, Finsch (nec Gray), Vög. der Südsee, p. 21 (1884) (Nova Britannia, Nova Guinea). - ? Ramsay, Tab. List, p. 23, no. 695 (1888).

? Sterna nigrirons, Masters, Pr. Linn. Soc. N. S. W. I, p. 63 (1875). - Ramsay, Tab. List, p. 23, no. 699 (1888).

Sterna longipennis, Grant, P. Z. S. 1888, p. 204 (Rubiana, Isole Salomone).

Hab. in Ins. Salomonis (Woodford).

Io ho potuto esaminare due esemplari raccolti dal Finsch, l'uno nella Nuova Britannia (no. 688) e l'altro presso Port Moresby nella Nuova Guinea (no. 1252), ora conservati nella Collezione Turati ; essi non sono in abito perfetto, ma sono similissimi ad un esemplare di Halmahera della Collezione Bruijn (Orn. III, p. 440, a), che è stato determinato dal Saunders, come appartenente alla S. longipennis, Nordm.

\section{(940) Sterna dougalli (Mont.).}

Sterna gracilis, Ramsay, Pr. Linn. Soc. N. S. W. VII, p. 41 (1882) (Robinson Island, Isole Salomone, Morton).

\section{(941) Sterna melanauchen, Tемm.}

Sterna melanauchen, Finsch, Vög. d. Südsee, p. 21 (Nova Britannia) (1884). - Meyer, Zeitschr. f. ges. Orn. I, pp. 197, 216 (Timorlaut, Riedel) (1884). - Ramsay, Tab. List,-p. 23 (1888).

(942) Sternula sinensis (GM.).

Sterna sinensis, Finsch, Vög. d. Südsee, p. 22 (Tova Britannia) (1884).

Sternula sinensis, Ramsay, Tab. List, p. 23 (1882!

(943) Onychoprion fulginosum (Gr.).

Sterna fulginosa, Ramsay, Tab. List, p. 23 (1888).

(944) Onychoprion anaesthetus (ScoP.).

Onychoprion anaesthetus, Sclat, P. Z. S. 1883, pp. 52, 200 (Moloe, Timorlaut)。 - Forb., P. Z. S. 1884 , p. 434 .

Sterna anaestheta, Ramsay, Tab. List, p. 23 (1888).

(946) Anous stolidus (Linn.).

Anous stolidus, Finsch, Vög. d. Südsee, p. 22 (.lova Britannia) (1884). - Ramsay, Tab. List, p. 23 (1888).

(947) Anous melanogenys, G. R. Gr.

Anous melanogenys, Ramsay, Tab. List, p. 23 (1888).

(948) Anous leucocapillus, Goodd.

Anous leucocapillus, Grant, P. Z. S. 1887, p. 333. - Ramsay, Tab. List, p. 23 (1888). - Salvad, Ann. Mus. Giv. Gen. (2) X, p. 834 :1891) (Isoln Goodenough).

Hab. in mare prope Ins. Salomonis (Woodford) (1).

(1) Il Ramsay (Tab. List, p. 23) annovera ancora la Nuova Guinea meridionale-orientale fra luoghi abitati dallo Anous tenuirostris (Temm.), senza convalidare l'asserzione con prove di fatto.

[86] 
(950) Puffinus leucomelas (Темм.).

Puffinus leucomelas, Pleske, Bull. Acad. Petersb. XXIX, p. 539 (Ternate) (1884).

(952) Puffinus sphenurus, Godrd.

Puffinus sphenurus, Pleske, Bull. Acad. Petersh. XXIX, p. 539 (Ternate) (1884). - Ramsay, Tab. List, p. 24 (1888).

Questa specie non si conosceva di Ternate.

(956) Podicipes gularis, Goold.

Podiceps novae-hollandiae, Ramsay, Tab. List, ‥ 22 (1888.

(957) Podicipes tricolor, G. R. GR.

Podiceps tricolor, Pleske, Bull. Acad. Petersb. XXIX, p. 540 (Ternate) (1884).

\section{ORDO STRUTHIONES}

(960) Casuarius galeatus, Lath.

Casuarius galeatus, Sclat., Voy. Chall., Birds, p. 64 (1881).

(962) Casuarius uniappendiculatus, Biтtн?

Casuarius uniappendiculatus, Meyer, Zeitschr. f. ges Orn. I, p. 296 (uovo) (1884).

Il Meyer attribuisce dubitativamente a questa specie un uovo di Casuario raccolto presso Sekaar nella Nuova Guinea meridionale-occidentale.

\section{Casuarius sp.}

Casuarius sp., Mejer, Zeitschr, f. ges. Orn. I, p. 296 (uova ex Aru, Ribbe).

Il Meyer descrive uova di Casuario, raccolte nelle isole Aru dal Ribbe, ma ignora se appartengano al $C$. bicarunculatus, Sclat. od al $C$. beccarii, Sclat.

\section{Casuarius, sp.}

Casuarius (beccari ?), Ramsay, Pr, Linn. Soc. ํ. S. W. VIII, p. 27 (1883) (uovo).

Non sappiamo finora che il $C$. beccarii si estenda nella Nuova Guinea orientalemeridionale, d'onde proviene l'uovo descritto dal Ramsay; è più probabile che esso appartenga al $C$. picticollis, Sclat. 


\section{A P P E N D C E}

\section{(8) Cuncuma leucogaster (Grr.).}

Haliaetus leucogater, Tristr., Ibis, 1889, p. 558.

\section{(1033) Urospizias woodfordi (SHARPE).}

Astur woodfordi, Ibis, 1889, p. 582.

Ho visto queste specie nel Museo Britannico.

(1035) Urospizias shebae (SHARPE).

Astur shebae, Ibis, 1889, p. 582.

Ho veduto anche questa specie nel Museo Britannico.

(43) Ninox assimilis, SALVAD. et D'ALB.

Ninox assimilis, Salvad., Ann. Mus. Civ. Gen. (2) IX, p. 556 (1890) (Rigo).

Sp. 1071 ( $\left.41^{\text {bis }}\right)$ Ninox goodenoviensis, DE VIs.

Ninox goodenoviensis, De Vis, Ann. Rep. Brit. New Guin. p. 58 (1890). - Id., Colon. Papers, no. 103, p. 107 (1809). - Id., Ibis, 1891, p. 27.

Notaeo, auricularibus et cauda brunneis, immaculatis; dorso, scapularibus tectricibusque alarum minoribus paullum rufescentibus; capite fusco-cinerascente; scapularibus maculis obtectis parvis albis praeditis; alis fuscis, remigibus primariis in pogonio interno superne, fasciis pallidis, inferne basin versus fasciis albis notatis; secundariis et tertiariis in pogonio interno albo fasciatis; cauda fusca unicolore, inferne pallidiore; plumis frontis lorique griseis, scapis nigris; gula brunnea; pectore summo rufo, albo vario; pectore ino et abdomine mis, plumis singulis maculis albis in utroque pogonio, majoribus in medio, minoribus ad apicem, notatis; tibiis albo aeruginosis; subcaudalibus aeruginosis, in medio lute fuscis; margine alarum albo; subalaribus rufis, majoribus fuscis, late albo transfasciatis; rostro fusco, culmine et apice albo-flavidis; pedibus (an potius digitis?) fusco-roseis (!); tarsis rufis, plumis in medio fuscis, tarsis omnino plumosis.

Long. tot. $0^{\mathrm{m}}, 285$; al. $0^{\mathrm{m}}, 222$; tarsi $0^{\mathrm{m}}, 031$.

Hab. Insula Goodenough (fide De Vis).

Secondo il De Vis questa specie arrebbe la coda unicolore come la $N$. theomacha: Io ho tradotto la descrizione del $\mathrm{De}$ Vis. 
Sp. 1172 (43 $3^{\text {bis }}$ Ninox rosseliana, Tristr.

Ninox rosseliana, Tristr., Ibis, 1889, ‥ 557.

N. superne fusco-brunnea, unicolor; tectricibus alarum in externo pogonio albo maculatis; remigibus fuscis sine maculis; cauda obscure brunneo fasciata; pectore et abdomine albidis, striis castaneis longitudinaliter variegatis ; 'tibiis et tarsis castaneis; rostro albiclo; pedibus pallidis (Tristram).

Long. tot. 11 poll. Angl. $\left(=0^{\mathrm{m}}, 279\right)$; alae $8.1 \quad\left(=0^{\mathrm{m}}, 205\right)$; caudae 5 $\left(=0^{\mathrm{m}}, 127\right)$; rostri $0.9\left(0^{\mathrm{m}}, 022\right)$; tarsi $1.3\left(=0^{\mathrm{m}}, 033\right)$.

Hab. in Ins. Rossel dicta (Thomson).

Dice il Tristram che questa specie è affine alla $N$. boobook, ma molto più piccola.

\section{(56) Cacatua triton (Tems.).}

Cacatua Trobriandi, Finsch, Samoafahrten, p. 208 (1888).

Cacatua triton, Salvad, Ann. Ilus, Civ, Gen. (2) IX, p. 552 (1890) (Rigo).

Nel luogo citato dimostrai clie la Cacatua della Nuova Guinea meridionaleorientale non è la specie australiana, come avera affermato il Ramsay, ma la $C$. triton, distinta per aver la pelle nuda intorno agli occhi celeste.

Il Finsch (in litt.) mi assicura che la C. trobriandi, descritta delle Isole Trobriand, non è diversa dalla $C$. triton.

(61) Gacatua ducorpsi, JACQ. ot Pucher.

Cacatua ducorpsi, Woodf., P. Z. S. 1888, p. 249.

(63) Nasiterna bruijni, SALVAD.

Nasiterna bruijni, De Vis, Ann. Rep. Brit. New Guin. p. 58 (1890).-Id., Colon. Papers, no. 103, p. 107 (1890! - Id., Ibis, 1891, p. 28.

Hab. Montibus Musgrave (MracGregor).

La presenza di questa specie, che si credera confinata nei monti Arfak, nella Catena Musgrave ad 8000 piedi di altezza, è cosa notevole; sarebbe impọtante di poter confrontare gli esemplari dei due luoghi.

(69) Nasiterna pusio, Sclat.

Nasiterna pusio, Tristr., Ibis, 1889, p. 557.

Hab. in Ins. St. Aignan dicta (Thomson).

(1043) Nasiterna aolae, Grant.

Nasiterna aolae, Ibis, 1889, p. 582.

(86) Gyclopsittacus diophthalmus (H. th J.).

Cyclopsittacus diophthalmus, Meyer, Ibis, 18\%0, p. 412 (Constantine Harbour, N. E. Jew Guinea).

7 T. Snlvodori. 
(1047) Cyclopsittacus edwardsi, Odst.

Cyclopsittacus edwardsi, Ibis, 1889, p. 583. - Neyer, Ibis, 1890, p. 412.

11 Meyer descrive una femmina di Constantine Harbour nel modo seguente:

La femmina differisce dal maschio principalmente per l'assenza del rosso sul: petto e sull'addome; inoltre le piume sul mezzo del pileo hanno gli apici azzurri, ed avanti alla fascia nera della cervice ve n'è una di colore oliva della stessa larghezza. L'Oustalet non descrive la fascia oliva nel maschio, nel quale il verde della testa è separato dal verde della cervice soltanto dalla fascia nera.

Lunghezza dell'ala $0^{\mathrm{m}}, 105$; della coda $0^{\mathrm{m}}, 056$; culmine del becco $0^{\mathrm{m}}, 020$; tarso $0^{\mathrm{m}}, 011$.

Sp. 1173 (90 $\left.90^{\mathrm{bi}}\right)$ Gyclopsittacus nigrifrons. RcHww.

Cyclopsittacus nigrifrons, Rchnw, Allg. Deutsche Orn. Gesell. zu Berlin, Bericht 1II, p.. 6 \{20 März, 1891j. - Id,, Journ. f. Orn. 1891, p. 217.

Mas. «Superne viridis, fronte nigra; genamu parte anteriore albida, parte posteriore. loris, temporibusque nigris; gula et colli lateribus pallide octuraceis; pectore sordide aurantiaco; gastraeo reliquo et subalaribus flavescente-viridibus; remigibus nigris, primariis pogonio externo, earum tectricibus et margine alarum superiore et inferiore cyancis, remigibus secundariis anterioribus pogonio externo virescente-caenuleis, posterioribus pogonio externo viridibus, ultimis pogonio interno macula celata flara ornatis; rostro nigro » (Reichenowo).

Long. tot. c. 140 , alae 90 , caudae 40 , rostri a fronte 15 , tarsi 12 , altitudo rostri $17 \mathrm{~mm}$.

Foemina. "Superne viridis; fronte, loris et temporibus nigris; genis, gula et colli lateribus albido flavidis; hoc colore ad pectus in colorem pallide ochraceum transcunte 》 (Reichenow).

Hab. Nova Guinea septentrionalis, ad flumen Augusta (Huirstein).

Il Reichenow fa notare che l'esemplare descritto come maschio, e che fu indicato dal collettore come femmina, non sembra in abito perfetto; inoltre egli dice che questa specie differisce dall'affine $C$. melanogcnys per le dimensioni notevolmente maggiori, pel' nero cupo della fronte e pel vessillo interno delle remiganti di un nero puro, senza il margine giallo che si trova in quella specie.

Sp. 1174 (95 $\left.{ }^{\text {bis }}\right)$ Geoffroyus sudestiensis, DE VIs.

Geoffroyus sudestiensis, De Vis, Ann. Rep. Brit. New Guin. p. 58 (1890). - Id., Colon. Papers, no. 103, p. 107 (1890). - Id., Ibis, 1891, p. 28.

Mas. Supra laete viridis, subtus pallidior; fronte, genis, mento, gula et auricularibus rubris; his lilacino tinctis; capite et collo antico pallide caeruleolilacino tinctis; vertice sub quamdam lucem flavicante; remigibus fuscis, prima caeruleo marginata, reliquis in toto pogonio externo et parte pogonii interni viridibus; macula culitali milla; subalaribus et axillaribus laete caeruleis: remigibus subtus fuscis; supracaulalibus et rectricibus mediis superne flavicanteviridibus, pogonio interno rectricum et latere inferiore caudae virescente-flavis; 
uropygio viridi; maxilla nubra, apice pallido, mandibula caerulescente fusca; pedibus fuscis.

Long. tot. $0^{\mathrm{m}}, 240$; al. $0^{\mathrm{m}}, 175$; caud. $0^{\mathrm{m}}, 100$; rostri $0^{\mathrm{m}}, 024$; tursi $0^{\mathrm{m}}, 015$.

$H a b$. Insula Sudest dicta (fide De Vis).

Secondo il De Vis questa specie si distingue dalle altre affini per la forte tinta gialla sulla testą, visibile soltanto sotto certe incidenze di luce, e per la mancanza della macchia cubitale (bruna).

(105) Eclectus pectoralis (P. L. B. MüLI.).

Eclectus polychlorus, North, Pr. Linn. Soc. N. S. W. (2) II, p. 445 (uovo, N. Gininea) (1887).

Eclectus polychloros, Woodf., P. Z. S. 1888, p. 249.

Eclectus cardinalis, De Vis (nec Bodd.), Ann. Rep. Brit. New Guin. p. 58 (1890) (Normanhy Island). - Id., Colon. Papers, nn. 103, p. 108 (1890; - Id, Ibis, 1891, p. 29

Eclectus pectoralis, Salvad., Ann. Mus. Civ. Ger. (2) IX, p. 558 (1890) (Rigo).

È impossibile che gli esemplari dell'Isola Normanby appartengano all' $E$. cardinatis, che è confinato nelle isole del gruppo di Amboina, e senza dubbio essi debbono essere riferiti all' $E$. pectoralis, che è la specie che dalla Nuova Guinea si estende fino alle Isole Salomone.

\section{Prioniturus platurus (Vieill.).}

Eclectus platurus, Schleg., Mus. P. B. Psittaci, Revue, p. 22 (1874).

Lo Schlegel annovera una femmina giovane di questa specie, uccisa addi 8 novembre 1864 nella Baia di Bara, sulla costa Nord-owest di Bouro, ed inviata al Museo di Leida dall'Hoedt. Tale cosa mi sembra poco probabile e credo che occorrano ulteriori prove per ammetterla.

(111) Lorius hypoenochrous, G. R. GR.

Lorius hypoinochrous, De Vis, Ann. Rep. Brit. New Gnin. p. 58 (1890) (St. Aignan; Rossel Island). - Id., Colon. Papers, no. 103, p. 108 (1890). - Id., Ibis, 1891, p. 29.

(123) Eos cardinalis (G. R. GR.).

Lorius cardinalis, Woodf., P. Z. S. 1888, p. 249.

(146) Oreopsittacus arfaki (MEYER).

Oreopsittacus arfaki, Jbis, 1889, p. 583.

(150) Charmosyna josephinae (FINSCH).

Charmosyna josephinae, Tristr., Cat. Coll. B. p. 74 (1889).

Il Tristram annovera nel suo Catalogo esemplari di questa specie raccolti dal D'Albertis, ma questa cosa non è esatta, giacchè nelle collezioni del D'Albertis questa specie non si trovò mai; il Tristram, da me interpellato, mi scrive che quegli esemplari non hanno il cartellino del D'Albertis, e sono di fattura probabilmente dei collettori del Bruijn.

(151) Guculus canoroides, S. MüLL.

Cuculus intermedius, Vahl. - Shelley, Cat. B. XIX, p. 252 (1891; 
(152) Guculus micropterus, Govid.

Guculus micropterus, Shell., Cat. B. XIX, p. 241 (1891).

(153) Cacomantis pallidus (LATH.).

Guculus pallidus, Shell., Cat. B. XIX, p. 261 (1891)

(154) Cacomantis flabelliformis ( (

Cacomantis flabelliformis, Shell., Cat. B. XIX, p. 266 (1891).

Lo Shelley ha ommesso di menzionare che questa specie si trova anche nelle Isole Aru.

(155) Gacomantis castaneiventris, Gould.

Gacomantis castaneiventris, part., Shell, Cat. B. XIX, p. 274 (1891!.

(1055) Cacomantis arfakianus, SaLVAD.

Gacomantis castaneiventris, part., Shell., Cat. B. XIY, p. 274 (1891).

(156) Gacomantis aeruginosus, SALVAD.

Gacomantis virescens, part., Shell., Cat. B. XIX, p. 274 (1891).

Lo Shelley unisce il mio C. aeruginosus di Buru, Ceram ed Amboina col $C$. virescens (Brügg.) di Celebes. Per quanto lievi, le differenze fra le due forme mi sembrano anche ora meritevoli di valore specifico.

(157) Cacomantis assimilis (G. R, GR.).

Gacomantis insperatus, Gould, P. Z. S. 1845, p. 19. - Shell., Cat. B. XIX, p. 273 (1891).

È singolare che lo Shelley, mentre identifica il $C$. assimitis (Gray) delle Isole Papuane e delle Molucche col Cuculus insperatus, Gould, d'Australia, non menzioni questa fra i luoghi abitati dal medesimo e non annoveri nessun esemplare australiano! Io credo che quella identificazione richieda ulteriori confronti prima di essere accettata.

Lo stesso Shelley (1. c., pp. 268, 272) riferisce al Cacomantis merulinus (Scop.) un esemplare indicato di Ternate, ma senza nome del collettore. Se la località è esatta, io sospetto che quell'esemplare appartenga al C. tymbonomus, od al C. assimitis, che si trovano ambedue in Ternate.

(158) Cacomantis tymbonomus (S. MüLL.).

Gacomantis variolosus (Horsf.). - Shelley, Cat. B. XIX, p. 272 (1891).

La preferenza data dallo Shelley al nome dell'Horsfield, fondato sopra un giovane esemplare, non mi pare giustificata.

(159) Rhamphomantis megarhynchus (G, R. GR.).

Ramphomantis megarhynchus, Shell, Cat. B. XIX, p. 329 (1891).

La collocazione di questa specie fra $\mathrm{i}$ generi IIicrodynamis e Scythrops, la quale cosa è stata fatta dallo Shelley, mi sembra poco naturale. 
(160) Misocalius palliolatus (Latir.).

Misocalius palliolatus, Shell., Cat. B. XIX, p. 279 (1891).

(161) Lamprococcyx meyeri, SALVAD.

Chalcococcyx meyeri, Shell, Cat. B. XIX, p. 293 (1891).

Lo Shelley annovera esemplari della Nuova Guinea orientale-meridionale.

(162) Lamprococcyx plagosus (Latн.).

Chalcococcyx plagosus Shell., Cat. B. XIX, p. 297 (1891).

(163) Lamprococcyx basalis (HoRsF.)

Chalcococcyx basalis, Shell., Cat. B. XIX, p. 294 ; 1891).

(1056) Lamprococcyx poliurus, SALtad.

Chalcococcyx poliurus, Shell., Cat. B. XIX, p. 296 (1891).

(164) Lamprococcyx poecilurus (G. R. Gr.).

Chalcococcyx poecilurus, Shell., Cat. B. XIX, p. 299 (1891).

Secondo lo Shelley, fra gli uccelli della Nuova Guinea e delle Isole Salomone si dovrebbe annoverare anche il Lamprococcyx malayanus (Raffl.) della penisola di Malacca, delle Isole della Sonda e delle Filippine (Cat. B. XIX, pp. 298, 299), col quale egli identifica il Chrysococcyx minutillus, Gould, P. Z. S. 1859, p. 128, di Port Essington nell'Australia. Ignoro se questa identificazione sia esatta, ed ignoro pure su cosa si fondi lo Shelley per asserire che il $L$. malayanus si trovi nella Nuora Guinea, del qual luogo non esiste alcun esemplare nel Museo Britannico; per asserire che esso si trovi nelle Isole Salomone, lo Shelley si fonda sugli esemplari di quelle Isole dal Grant (P. Z. S. 1888, p. 191) attribuiti al Lamprococcyx basalis. Non oso pronunciarmi in modo reciso intorno alla identificazione dello Shelley, e mi pare che converrà attendere nuove indagini intorno a tale argomento.

(165) Lamprococcyx poeciluroides, SALVAD.

Chalcococcyx poeciluroides, Shell., Cat. B. XIX, p. 301 (1891).

(166) Lamprococcyx ruficollis, Salvad.

Chalcococcyz ruficollis, Shell., (aal. B. XIX, p. 300 (1891).

(167) Lamprococcyx mysoriensis, SALVAD.

Chalcococcyx mysoriensis, Shell. Cat. B. XIX, p. 301 (1891).

(168) Lamprococcyx crassirostris, SALVAD.

Chalcococcyx crassirostris, Shell., Cat. B. XIY, p. 301 (1891).

(169) Surniculus muschenbroeki, MEYer.

Surniculus muschenbroeki, Shell., Cat. B. XIX, p. 230 , 1891). 
(170) Caliechthrus leucolophus (MüLL。).

Galiechthrus leucolophus, Meyer, Ibis, 1890, p. 413 (Constantine Harbour). - Shell, Cat. B: XIX, p. 225 (1891).

(174) Eudynamis orientalis (LiNs.).

Eudynamis orientalis, Shell., Cat. B. XIX, p. 322 (1891).

Io ho già espresso il dubbio che gli esemplari del gruppo di Halmahera non. appartengano all' $E$. orientalis, alla quale specie mi pare difficile che possano appartenere anche quelli di Lombock, come ha creduto lo Shelley.

(172) Eudynamis cyanocephala (LATII.).

Eudynamis cyanocephala, Shell., Cat. B. XIX, p. 324 (1891).

Io penso che questa specie sia confinata nell'Australia, nella Nuova Guinea meridionale e nelle Isole Aru e credo che gli esemplari di Dorei e di Goram, attribuiti alla medesima dallo Shelley, spettino rispettivamente all' $E$. rufiventer ed all' $E$. orientalis; anche l'esemplare di Timor, menzionato dallo Shelley, probabilmente appartiene ad una specie distinta.

(173) Eudynamis rufiventer (Less.).

Eudynamis rufiventer, Shell., Cat. B. XIX, p. 325 (1891),

A questa specie lo Shelley ha attribuito gli esemplari della Nuova Britannia, dell'Isola del Duca di York e delle Isole Salomone, i quali da me, dal Finsch e dal Grant sono stati riferiti invece all'E. cyanocephala.

(174) Urodynamis taitiensis (SPARM.).

Urodynamis taitiensis, Shell., Cat. B. XIX, p. 314 (1891).

\section{(175) Microdynamis parva (SALVAD。).}

Microdynamis parva, Shell., Cat. B. XIX, p. 328 (1891).

(176) Scythrops novae-hollandiae, Latr.

Scythrops novae hollandiae, Shell., Cat. B. XIX, p. 330 (1891).

(177) Centrococcyx medius (MüLL.).

Centropus javanicus, Shell., Cat. B. XIX, p. 354 (1891).

Io espressi già l'opinione che forse il $C$. medius delle Molucche non sia diverso dal C. javanensis, Dumont, delle Isole della Sonda, di Malacca e di Celebes. Lo Shelley ha concluso decisamente per la identità specifica. Egli avrebbe dovuto scrivere $C$. javanensis, e non $C$. javanicus, giacchè la prima maniera è quella adoperata dal Dumont, Dict. Sc. Nat. XI, p. 144.

(178) Nesocentor menebiki (GARN.).

Centropus menebiki, Shell., Cat. B. XIX, p. 336 (1891).

(179) Nesocentor aruensis, SAIVAD.

Centropus aruensis, Shell., Cat. B. XIX, p. 337 (1891. 
亡̀ affatto impossibile che l'esemplare giovane, indicato di Batchian dallo Shelley, appartenga a questa specie, se veramente di Batchian; se la località è esatta, esso dovrebbe appartenere al $N$. gotiath.

(180) Nesocentor violaceus (Q. ot G.).

Centropus violaceus, Layard, Ibis, 1880, p. 300 (New Britain). - Shell., Cat. R. XIX, P. 337 (1891).

(181) Nesocentor chalybeus, SALVAD.

Centropus chalybeus, Shell., Cat. B. XIX, p. 33 (1891).

(182) Nesocentor goliath (ForsteN).

Centropus goliath, Shell., Cat. B. XIX, p. 335 (1891).

(183) Nesocentor ateralbus (Lrss.).

Centropus ateralbus, North, Pr. Linn. Soc. N. S. W. (2) II, p. 444 (exgs, New Britain) (1887). Shell., Cat. B. XIX, p. 334 (1891).

(184) Nesocentor milo (Gould).

Centropus milo, Shell., Cat. B. XIX, p. 335 (1891).

(185) Polophilus spilopterus (G. R. GR.).

Centropus spilopterus, Shell., Cat. B. XIX, p. 338 (1891).

(186) Polophilus bernsteini (ScHLeg.).

Centropus bernsteini, Shell, Cat. B. XIX, p. 338 (1891).

(187) Polophilus nigricans, SALVAD.

Polopbilus nigricans, Salvad., Ann. Mus. Civ. Gen. (2) I, p. 559 (1890) (Rigo); X, p. 803 (1891).

Centropus nigricans, Shell., Cat. B. XIX, p. 339 (1891).

(188) Rhytidoceros plicatus (PenN.).

Rhytidoceros plicatus, Tristr. Ibis, 1889, p. 557. - De Vis, Ann. Rep. Brit. New Guin. p. 58 (1890) (0wen Stanley Range). - Id., Colon. Papers, no. 103, p. 108 (1890). - Id., Ibis, 1891, p. 29.

Sp. 1175 (189bis) Merops salvadorii, MEYER.

? Merops ornatus, Layard, Ibis, 1880, p. 300 (New Britain.). - North (nec Lath.), Pr. Linn. Soc. N. S. W. (2) II, p. 441 (New Britain) (1882).

Merops philippinus, Neyer (nec L.), lbis, 1890, p. 413 (New Britain).

Merops Salvadorii, Meyer, Ibis, 1891, p. 294.

Il Meyer dice di possedere un giovane esemplare di questa nuova specie proveniente dalla Nuova Britannia, e di averlo confrontato con un giovane del II. phitippinus di Macassar (Celebes), dal quale differisce per diversi rispetti.

« Il colorito generale dell'esemplare della Nuova Britannia è meno azzurroverde, di un verde-giallognolo e piủ chiaro; la fronte è giallognola ed il colore verde delle parti inferiori appare tinto del bruno della gola; le cuopritrici della coda sono di color azzurro più chiaro e le timoniere hanno una tinta verde-olivacea; la base delle 
remiganti è bruna, ed il colore cannella sulla faccia inferiore della prima remigante è più esteso. Ma forse il migliore carattere differenziale è la mancanza del colore verde sulla piegatura dell'ala, che è fulviccio cannella dalla piegatura alla remigante spuria e dello stesso colore delle cuopritrici inferiori delle ali, laddove nel $\lambda I . p 7 i$ lippinus il margine verde ha una larghezza di 2 a 3 millimetri inferiormente. Nel $M I$. ornatıs, che ha il becco più corto, quel margine è molto stretto; per altri rispetti il II. ormatus non ha niente da fare colla specie della Nuova Britannia. Nel caso che la mancanza del verde sul margine dell'ala nell'esemplare della Nuova Britannia fosse dovuto ad immaturità del medesimo, è probabile che l'uccello adulto abbia quel margine come il $M$. ornatus $\gg$.

Dimensioni: Lunghezza totale 220 millim.; ala 120 ; coda 90 ; timoniere mediane 94 ; culmine del becco 32.

Senza vedere l'esemplare descritto dal Meyer, non riesco a farmi un'idea chiara di questa specie, che egli ha avuto la cortesia di dedicare a me.

Pare probabile che a questa specie si debbano riferire gli esemplari della Nuova Britannia e le uova che il North riferisce al $M$. ornatus, ma che egli nota essere simili a quelle della forma Australiana, ma più piccole!

(197) Ceyx solitaria, Teмs.

Ceyx solitaria, De Vis, Ann. Rep. Brit. Llew Guin. p. 58 (1890) (Kormanby Island). - Id., Colon. Papers, no. 103, p. 108 (1890). - Id., Ihis, 1891, p. 29.

Sp. $1176\left(207^{\mathrm{bi}}\right)$ Tanysiptera rosseliana, Tristr.

Tanysiptera rosseliana, Tristr., Ibis, 1889, p. 557.

Tanysiptera galatea, De Vis. (nec Gray), Ann. Rep. Brit. New Guin. p. 58 (1890) (Rossel Island). - Id., Colon. Papers, no. 103, p. 108 (1890). - Id., Ibis, 1891, p. 29.

T. supra saturate caerulea; pileo toto usque ad occipitem, et tectricibus alarum superioribus laete caemleis; lateribus capitis caeruleis dorso concoloribus; remigibus nigris, in pogonio externo dorsi colore caeruleo marginatis; tergo inferiore, cauda et toto corpore subtus purissime albis; cauda spatulata; spatula alba, parte rachidis angustiore duarum mediarum rectricum ultra ćaudam caerulea; rostro rubro; pedibus nigris.

Long. tot. 15 poll. Angl. $\left(=0^{\mathrm{m}}, 381\right)$; alae $4.35\left(=0^{\mathrm{ma}}, 110\right)$; caudae 5.5 $\left(=0^{\mathrm{m}}, 139\right)$; caud. cum rectr. med. $10.6\left(=0^{\mathrm{m}}, 269\right)$; rostri $1.6\left(=0^{\mathrm{m}}, 040\right)$.

Hab. in Ins. Rossel (Ins. Ludovicianis) (Thomson).

Il Tristram dice che nella disposizione dei colori delle due timoniere mediane questa specie differisce da tutte le altre; egli spiega quella disposizione dicendo che Ia colla è bianca, e che gli steli delle due timoniere mediane hanno un sottilissimo margine azzurro terminante ove comincia la dilatazione della spatola.

Ho esaminato un esemplare tipico di questa specie, la quale si distingue facilmente dalle affini pel sopraccoda e per le timoniere laterali interamente bianche, senza margini azzurri. Le due timoniere mediane sono azzurre soltanto nella parte ristretta, e non marginate di azzurro, come dice il Tristram.

Questa specie è stata scambiata dal De Vis per la T. galatea. 
(213) Cyanalcyon leucopygia (VERr.).

Gyanalcyon leucopygialis, Ramsay, Records Austr. Mus. I, Part I, p. 3 (1890).

Hab. Ins. Howla, Ins. Salomonis.

\section{(219) Sauropatis saurophaga (Gould).}

Sauropatis saurophaga, Tristr., Ibis, 1889, p. 557.

Halcyon albicilla, Ramsay (nec Cuv.), Hec. Austr. Mus. I, pt. 1, p. 4 (1890) (Howla Isl.).

Halcyon saurophaga, De Vis, Ann. Rep. Brit. New Guin. p. 58 (East Island). - Id., Colon. Papers, no. 103, p. 108 (1890). - Id., Ibis, 1891, p. 29.

$H a b$, in Ins. East dicta (Thomson).

(224) Syma torotoro, LEss.

Syma torotoro, Tristr., Ibia, 1889, p. 557. - Salvad., Ann. Nus. Civ. Gen. (2) IX, p. 561 (1890) (Rigo); X, p. 805 (1891).

$H a b$. in Ins. Fergusson (Thomson).

\section{(225) Sauromarptis gaudichaudi (Q. et G.).}

Dacelo gaudichaudi, Tristr., Ibis, 1889, p. 557.

Sauromarptis gaudichaudi aruensis, Meyer, Ibis, 1890, p. 415.

Il Meyer afferma che gli esemplari delle isole Aru si distinguono per un'area bianca sul dorso, la quale in vero si trova anche in quelli della Nuova Guinea.

\section{Sp. 1177 (225 is). Sauromarptis kubaryi, Merer.}

Sauromarptis kubaryi, Neyer, Ibis, 1890, p. 414.

«Fem. S. gaudichaudi similis, sed alarum tectricibus omnino cyanescenticaeruleis, uropygio clariore et intense cyanescente, dorso albo et corpore subtus caudaque cinnamomeis, diversa.

Long. al. $0^{\mathrm{m}}, 130$; caud. $0^{\mathrm{m}}, 091$; rostri (culm.) $0^{\mathrm{m}}, 059$; tarsi $0^{\mathrm{m}}, 016 》$,

Hab. Sinu Constantini, Nova Guinea Septentrionali (Kubary).

Non conosco questa specie altro che per la descrizione del Meyer.

(227) Dacelo intermedius, Salvad.

Dacelo intermedius, Salvad, Ann. Mus. Civ. Gen. (2) IX, p. 562 (1890) (Rigo).

(228) Melidora macrorhina (Less.).

Melidora macrorhina, Meyer, Ibis, 1890, p. 413. - Salvad., Ann. Mus. Giv. Gen. (2) X, p. 805 (1891).

Il Mejer (1. c.) ha cercato di far rivivere la $M$. goldiei, Ramsay, che egli considera come distinta dalla forma settentrionale occidentale, dicendo che la prima si distingue per avere le remiganti, le timoniere e le macchie delle parti superiori più verdognole; io ho confrontato recentemente due femmine della Nuova Guinea meridionale-orientale, raccolte dal Loria, con altre di Audai, e non sono riuscito a trovare le differenze menzionate dal Meyer; la sola differenza sensibile l'ho trovata nelle dimensioni del becco, alquanto minori negli esemplari della Nuova Guinea meridionale-orientale. 
(230) Eurystomus pacificus (Latн.).

Eurystomus pacificus, Tristr., Ibis, 1889, p. 556.

Sp. $1178\left(232^{\mathrm{bis}}\right)$ Eurystomus salomonensis, SHARPE.

Eurystomus salomonensis, Sharpe, P. Z. S. 1890, p. 552.

Eurystomus salomonensis, Dress., Ibis, 1891, p. 102.

« $\mathbf{E}$. similis $\mathbf{E}$. crassirostri, sed rostro omnino mbro, culmine minime nigro terminato distinguendus.

Long. tot. 12.0 unc. angl. $\left(=0^{\mathrm{m}}, 304\right)$; culm. $1.45\left(=0^{\mathrm{m}}, 036\right)$; al. 7.7 $\left(=0^{\mathrm{m}}, 195\right)$; caud. $5.2\left(=0^{\mathrm{m}}, 132\right)$; tarsi $0.7\left(=0^{\mathrm{m}}, 17\right) \gg$ (Sharpe).

$H a b$. in Insulis Salomonis.

(284) Podargus papuensis, Q. et G.

Podargus papuensis, Tristr., Ibis, 1889, p. 556.

(285) Podargus ocellatus, Q. et G.

Podargus ocellatus, De Vis, Ann. Rep. Brit. New Guin. p. 58 (1890) (Fergusson Island, Normanby Island). - Id., Colon. Papers, no. 103, p. 108 (1890). - Id., Ibis, 1890, p. 29.

Le Isole Fergusson e Normanby sono luoghi affatto nuovi per questa specie.

Sp. 1179 (241 bis) Aegotheles loriae, Salvad.

? Aegotheles wallacei, De Vis, Ann. Rep. Brit. New Guin. p. 58 (1890) (Mt. Kolwald at 2600 feet, Musgrave Range, 4000 fee ). - Id., Colon. Papers, p. 108 (1890). - Id., Ibis, 1891, p. 29.

Aegotheles loriae, Salvad., Ann. Mus. Civ. Gen. (2) IX, p. 564 (1890).

Aegotheles Ae. wallacei simillimus, sed valde major, subalaribus obscurioribus, vix albicantibus, majoribus omnino griseo-nigris, maculis albis apicalibus destitutis.

Long. tot. $0^{\mathrm{m}}, 260$; al. $0^{\mathrm{m}}, 130$; caud. $0^{\mathrm{m}}, 120$; rostri culm. $0^{\mathrm{m}}, 012$; tarsi $0^{\mathrm{m}}, 022$.

Hab. Rigo, Nova Guinea meridionali-orientali (Loria).

Molto probabilmente a questa specie, e non al vero $A$. wallacei della Nuova. Guinea settentrionale-occidentale, si debbono riferire gli esemplari raccolti, durante la spedizione del MacGregor, sul monte Kolwald a 2600 piedi e sui Monti Musgrave a 4000 piedi di altezza.

(251) Macropteryx mystacea (Less.).

Macropteryx mystacea, North, Pr. Linn. Soc. N. S. W. (2) II, p. 441 (egg) (1887). - Meyer, Ibis, 1890, p. 416 (New Britain). - De Vis, Ann. Rep. Brit. New Guin. p. 58 (1890) - Id.

Colon. Papers, no. 103, p. 108 (1890). - Id., Ibis, 1891, p. 29.

Dendrochelidon mystacea, Rams., Rec. Austr. Mus. I, p. 5 (1890) (Howla Isl.).

(253) Collocalia fuciphaga (Tunb.).

Gollocalia fuciphaga, Tristr., lbis, 1889, p 556. - De Vis, Ann. Rep. Brit. New Guin. p. 58 (1890) (St. Aignan). - Id., Colon. Papers, no. 103, p. 108 (1890) - Id., Ibic, 1891, p. 29.

$H a b$. in Ins. St. Aignan dicta (Thomson). 
Gen. PETRoGHELIDON, $\mathrm{U}_{\Delta \mathrm{B}}$.

Anthrochelidon, Baldam., Journ. f. Orn. 1869, p. 406. - . . . H. nigricons, Vieill.

Il nome generico Anthrochetidon non è menzionato dallo Sharpe e neppure è annoverato dal Waterhouse.

(259) Petrochelidon nigricans (Vieill.).

Anthrochelidon nigricans, Baldam., Journ, f, Orn. 1869, p. 406.

(260) Peltops blainvillei (L. ot G.).

Peltops blainvillei, Meyer, Ibis, 1890, p. 416 . Constantine Harhour\%.

Sp. 1180 (991 ${ }^{\text {bis }}$ ) Monarcha florenciae (SIIARPE).

Piezorhynchus florenciae, Sharpe, Ibis, 1890, p. 207.

Supre griseo-caemlescens, tectricibus alarum minoribus concoloribus; tectricibus reliquis remigibusque fuscis, griseo marginatis; rectricibus nigricantibus, griseo marginatis; capite obscuriore, genis et gula nigricantibus; fascia superciliari postica Tatissima, macula suboculari albis; auricularibus griseo schistaceis; collo imo antico et pectore summo griseo-caerulescentibus; pectore imo, abdomine et subcaudalibus laete rufo-cinnamoneis; tibiis griscis; subalaribus griseo-caeruleis, cinnamoneo tinctis; "rostro et pedibus caeruleo-ardesiacis; iride nigra » (Woodford).

Foem. MIari similis, sed genis et gula haud nigricantibus et fascia superciliari a7ba mulla.

Long. tot. $0^{\mathrm{m}}, 160-0^{\mathrm{m}}, 152$; al. $0^{\mathrm{m}}, 078-0^{\mathrm{m}}, 076$; caud. $0^{\mathrm{m}}, 062$; rostri culm. $0^{\mathrm{m}} .016$; tarsi $0^{\mathrm{m}}, 017$.

Hab. Ins. Salomonis, Ins. Rubiana (Woodford).

Dice lo Sharpe che questa specie a primo aspetto somiglia al $\boldsymbol{M}$. inornatus pel colore grigio delle parti superiori e rossigno delle inferiori, ma che il sopracciglio bianco la distingue facilmente; inoltre le fitte e brevi pinme frontali la fanno annoverare nel sottogenere Piezorhynchus; fra le specie di questo la più affine è il $P$. richardsi, ma ne differisce per avere di color grigio le parti che nel $P$. richardsi sono nere, e per avere soltanto il sopracciglio e non tutto l'occipite bianco.

Nel maschio la larga fascia sopraccigliare bianca si allarga sotto l'occipite e si estende sui lati del collo.

Sp. 1181 (261 $\left.{ }^{\text {ter }}\right)$ Monarcha leucophthalmus (RAMSAT).

Pomarea leucophthalmus, Rams., Rec. Austr. Mus. I, p. 4 (1890).

Capite, collo, gula, pectore et notneo toto nitide nigris; plumis gulae foeminae elongatis (?!); ala et cauda subtus fuscis; pogonio interno remigum primariarum sordide cinereo-albidis; abdomine, subalaribus et subcaudalibus saturate castaneis, macula semilunari ante oculos alba in foemina (?), rufa in mare; rostro nigro, tomiis albo-corneis; pedibus nigris. 
Long. tot. unc. angl. $6.6-6.8\left(=0^{\mathrm{m}}, 162-0^{\mathrm{m}}, 172\right) ;$ alae $5.25-5.5$ $\left(=0^{\mathrm{m}}, 133-0^{\mathrm{m}}, 139\right)$; caud. $3\left(=0^{\mathrm{m}}, 026\right)$; tarsi $0.7-0.81\left(=0^{\mathrm{m}}, 017-0^{\mathrm{m}}, 020\right)$; rostri $7.0-0.75\left(=0^{\mathrm{m}}, 017-0^{\mathrm{m}}, 019\right)$.

Hab. Ins. Howla, Ins. Salomonis.

Dice il Ramsay che questa specie somiglia alla $P$. castaneiventris (Verr.), dalla quale differisce per la macchia semilunare bianca avanti agli occhi. Secondo lo Sharpe essa sarebbe identica colla sua $P$. crythrosticta, ed il colore bianco della macchia semilunare avanti gli occhi sarebbe doruto a scoloramento prodotto dalla conservazione nell'alcool dell'esemplare tipico femmina.

(271) Monarcha melanopterus, G. R. GR.

Monarcha melanopterus, De Vis, Ann, Rep. Brit. New Guin. p. 58 (1890) (Sud-est Island; Rossel Island). - Id., Colon. Papers, no. 103, p. 108 (1890). - Id., Ibis, 1891, p. 30.

(283) Monarcha chalybeocephalus (GARN.).

Piezerhynchus chalybeocephalus, North, Pr. Linn. Soc. N. S. W. (2) II, p. 442 (eggs , New Britain) (1883).

\section{Sp. 1182 (291 ter) Arses orientalis, SALVAD}

Arses orientalis, Salvad, Ann. Nus. Civ。 Gen. (2) IX, p. 566 (1890); X, p. 808 (1891).

MLas mari $\mathbf{H}$. henkei simillimus, foemina foeminae $\boldsymbol{\Lambda}$. telescophthalmae similis, sed abdomine et subcaudalibus albidis, rufescente tinctis diversa. Statura A. telescophthalmae.

Hab. Rigo et Kemp Weltch, Nova Guinea meridionali-orientali (Loria).

(294) Sauloprocta melaleuca (Q. et G.).

Sauloprocta tricolor, Ramsay, Rec. Austr. Mus. I, p. 4 (1890).

Hab. Howla, Ins. Salomonis.

(305) Rhipidura setosa (Q. et G.) (?).

Rhipidura setosa, De Vis, Ann. Rep. Brit. New Guin. p. 59 (1890) (St. Aignan). - Id., Colon. Papers, no. 103, p. 108 (1890). - Id., Ibis, 1891, p. 30. - Salvad., Ann. Mus. Civ. Gen. (2) X, p. 829 (Isola Goodenough (1891).

Il De Vis menziona certi caratteri distintivi degli esemplari dell'Isola di St. Aignan, i quali perciò dorranno essere confrontati con esemplari tipici ; non è improbabile che essi siano da riferire alla $R$. finschi, Salvad.

(995) Rhipidura finschi, SALtad.

Rhipidura finschi, Meyer, Ibis, 1890, p. 416 (Duke of York group, Brown).

\section{(307) Rhipidura cinerea, WALL.}

Rhipidura lenzi, Forb. (nec Blas. ?), P. Z. S. 1884, p. 431 (Amboina).

Ho esaminato nel Museo Britannico l'esemplare di Amboina attribuito dal Forbes alla $R$. lenzi; esso è un giovane e, secondo me, non v'ha dubbio che esso appar- 
tenga alla $R$. cinerea. Dopo ciò la presenza della $R$. Tenzi, Blas. in Amboina non ha più fondamento, a meno che essa, come io dubito, non sia identica colla $R$. cinerea, e per errore sia stata indicata di Celebes.

\section{Sp. 1183 Rhipidura auricularis, De VIS.}

Rhipidura auricularis, De Vis, Ann. Rep. Brit. New Guin. p. 59 (1890) - Id., Colon. Papers, no. 103 p. 108 (1890). - Id., Ibis, 1891, p. 30.

Supra fumoso-grisea; capite fusco-brumeo; loris, fucie, auricularibusque anterioribus nigris; auricularibus posterioribus elongatis albis; fascia supercitiari lata alba; gulá alba, plumarum basi nigra, mento fere nigro; fascia pectorati transversa lata nigra, a gula alba abmepte distincta; pectore inferiore in medio et abdomine fulvescentibus, illo nigro vario; pectoris, abdominisque lateribus obscure griseis; subcaudatibus albis, basi nigra; tectricibus alarum nigris; minoribus albo punctulatis, mediis albo terminatis, fasciam alarem transversam formantibus, majoribus maculis latis albis, fasciam latam transalarem formantilus; remigibus obscure brunneis; cauda fusco-nigra, rectricum trium extimarum apice stricto albo: axillaribus interioribus albis, basi nigra; subalaribus albis, nigro variis; remigibus subtus cinereo fuscis; primariis griseo marginatis, secundariis et tertiariis fulvescente-griseo marginatis; rostro obscure fusco, mandibula alba, apice fusco; pedibus nigris.

Long. tot. $0^{\text {m }}, 145$; al. $0^{\text {m}}, 082$; caud. $0^{\text {ma }}, 090$; rostri culm. $0^{\text {m }}, 006$; tarsi $0^{\mathrm{m}}, 016$.

\section{Hab. in Montibus Musgrave.}

Il De Vis crede che le specie più affini alla presente siano la $R$. javanica e la $R$. nigritorquis delle Filippine; essa fu trovata all'altezza fra $i 7000$ ed i 9000 piedi.

\section{Sp. 1184 (334 $\left.4^{\text {bis}}\right)$ Poecilodryas? sigillata, DE Vis.}

Puecilodryas? sigillata, De Vis, Ann. Bep. Brit. New Guin. p. 59 (1890). - Id., Colon. Papers, no. 103 , p. 109 (1890). - Id., Ibis, 1891, p. 31.

Ad. Omnino nigra, plaga alari alba, in medio nigra, basim remigum tertiarum interiorum occupante, excepta; rostro et pedibus nigris.

Long. tot. $0^{\mathrm{m}}, 150$; al. $0^{\mathrm{m}}, 102$; caud. $0^{\mathrm{m}}, 072$; rostri culm. $0^{\mathrm{m}}, 009$; tarsi $0^{\infty}, 028$.

Juv. Supra brunneo-rufa, striis scapalibus pallidis, et areis nigris notata; remigibus tertiariis ultimis stria scapnli lata albida notatis; uropygio lacte mifo; cauda fusco-brunnea, tectricibus extimis laeviter rufo marginatis; plumis latorum abdominisque in nigrum colorem transeuntibus; gula et pectore striis pallidis, apicem versus latioribus, ornatis; ala caudaque subtus cinerco-brunneis; tectricibus alarum castaneo-brunneis, maculis apicalibus fulvescente-albis, notatis; fascias duas alares formantibus:- subalaribus fuscis; rostro perlibusque fusco-brunncis.

Hab. in Monte Victoria, Nova Guinea meridionali-orientali. 
Questa specie fu trovata sul monte Vittoria della Catena degli Owen-Stanley a 13 mila piedi di altezza.

Sembra che essa sia affine alla $P$. aethiops, Sclat. della Nuova Britannia.

(344) Microeca laeta, SALVAD.

Microeca laeta, De Vis, Ann. Rep. Brit. New Guin. p. 59 (1890) (Nusgrave Range). - Id., Colon. Papers, no. 103, p. 109 (1890). - Id., Ibis, 1890, p. 31.

Gli esemplari, che dal De Vis vengono attribuiti a questa specie, furono raccolti sui Monti Musgrave fra i 7000 ed i 9000 piedi di altezza; essi dorranno essere confrontati col tipo di Wandammen.

(364) Machaerorhynchus xanthogenys, G. R. GR.

Machaerorhynchus xanthogenys, Salvad., Ann. Nus. Civ. Gen. (2) X, p. 809 (1891)

L'esame fatto di un esemplare conservato nel Museo Britannico, raccolto dal Forbes presso Sogeri, e di altri esemplari della Nuova Guinea orientale-meridionale, raccolti dal Loria, non mi lascia più alcun dubbio intorno agli esemplari del genere Machaerorhynchus di detta regione, che dal Ramsay furono attribuiti al MI. flaviventris, Gould, d'Australia, laddove essi spettano invece, come io avevo sospettato, al $\boldsymbol{M}$. xanthogenys.

\section{Sp. 1185 (372 $\left.{ }^{\text {bis }}\right)$ Todopsis kowaldi, De VIs.}

Todopsis kowaldi, De Vis, Ann. Rep. Brit. New Guin. p. 59 (1890). - Id., Culon. Papers, no. 103, p. 109 (1890). - Id., lbis, 1891, p. 31.

Mas. jur. (?). Capite nigro, plumis caemleo terminatis, fasciam coronalem formantibus, circundato; auricularibus inferioribus obscure brunneis, superioribus elongatis, albis, ad apicem caenuleo tinctis; corpore supra olivaceo-brunneo; alis brunneis, tectricibus obscurioribus, mediis et majoribus nonnullis fulvo terminatis; remigibus exterius flavescenti-brunneo marginatis; cauda brunnea, inferius pallidiore; gastrdeo genisque fulvis; gula fere alba; subcaudalibus rufis, abdominis lateribus et tibiis olivaceo-brunneis; margine alarun, axillaribus et subalaribus isabellinis; remigibus subtus cinereis, margine interno rufescente; rostro obscure brunneo, mandibulae basi pallide flava; pedibus brumeis.

Long. tot. $0^{\mathrm{m}}, 155$; al. $0^{\mathrm{m}}, 085$; caud. $0^{\mathrm{m}}, 060$; rostri culm. $0^{\mathrm{m}}, 014$; tarsi $0^{\mathrm{m}}, 029$.

Hab. (?).

La località di questa specie non è menzionata dal De Vis, il quale fa notare com'essa sia affine alla $T$. wallacei, ma ne differisca per essere molto più grande e per avere le piume auricolari superiori bianche e tutto il corpo bruno e fulvo.

\section{Sp. 1186 (378 $\left.{ }^{\text {bis}}\right)$ Graucalus longicauda, DE VIS.}

Graucalus longicauda, De Vis, Ann. Bep. Brit. dew Guin. p. 59 (1890). - Id., Colon. Papers, no. 103, p. 110 (1890). - Id., Ibis, 1891, p. 32.

Mas. Capite, collo, mento gulaque, hac circumscripte, nitide nigris; alis et cauda nigris; dorso, uropygio, supracaudalibus, pectore, abdomine et subcauda- 
Tibus griseis; pogonio extenno remigum tertiarium ultimarum, tectricibusque alarum superioribus cinereo-griscis; subalaribus cinereo-griscis, obscurins marginatis; rostro et pedibus nigris.

Long. tot. $0^{m}, 330$; al. $0^{\mathrm{m}}, 180$; caud. $0^{\mathrm{m}}, 180$; rostri $0^{\mathrm{m}}, 022$; tarsi $0^{\mathrm{m}}, 028$.

$H a b$. in Montibus Musgrave.

Questa specie fu trovata all'altezza fra i 7000 ed i 9000 piedi. Non mi pare ammessibile che essa possa essere identica col Gr. Tettiensis, Meyer, o col Gr. timorTaoensis, Meyer, essendo queste specie forme rappresentanti e confinate rispettivamente nell'Isola Lettié e nel gruppo di Timorlaut.

(389) Graucalus pusillus, RAMSAY.

Edoliisoma salomonensis, Ramsay, Rec. Austr. Nus. I, p. 4 (1890).

Hab. Howla, Ins. Salomonis.

(414) Artamus leucogaster (VALENC.).

Artamus leucogaster, Sharpe, Cat. B. XIII, p. 3 (1890).

(1090) Artamus muschenbroeki, MEYER.

Artamus leucogaster, part., Sharpe Cat. B. XIII, p. 5 (1890):

(415) Artamus maximus, Merer.

Artamus maximus, Sharpe, Cat. B. XIII, p. 8 (1890).

(416) Artamus insignis, Sclat.

Artamus insignis, Sharpe, ('at. B. XIII, p. 11 (1890).

(1092) Dicruropsis propinqua (Tristr.).

Dicruropsis propinqua, Salvad., Ann. Mus. Giv. Gen. (2) X, p. 829 (Isola Fergusson) (1891).

(1093) Gracticus louisiadensis, Tristr.

Strepera rosa-alba, De Vis, Ann. Rep. Brit. New Guin. p. 59 (1890) (Sud-est Island). - Id., Colon. Papers, no. 103, p. $110(1890)$ - - Id., Ibis, 1891, p. 33.

Non credo che vi possa essere dubbio intorno alla identificazione della Strepera rosa-alba, De Vis, colla specie del Tristram, il tipo della quale proviene dallo stesso luogo.

(486) Rhectes brunneiceps, Salvad.

Rhectes brunneiceps, Sălvad., Ann. Nus. Civ. Gen. (2), X, p. 813 (Bara-Bara) (1891).

Sp. $1186\left(448^{\text {bis }}\right)$ Colluricincla (?) discolor, DE VIS.

Colluricincla discolor, De Vis, Ann. Rep. Brit. New Guin. p. 60 (1890), - Id., Colon. Papers no. 56, p. 111 (1890). - Id., Ibıs, 1891, p. 33.

Mas. Dorso, scapularibus, uropygio, supracaudatibus, margine remigum secundariarum, pogonio externo remigum tertiarum et margine rectricum viridi-olivaceis, uropygio flaro tincto; capite, collo et cerrice brunneis; alis et canda urunneis; 
gastraco albo-fuscescente; mento, gula ct abdomine imo medio albis; Tateribus brunnescente-flavicantibus; subcaudalibus pallide citrino-flavis; plumis gulae imae striis fuscis in medio notatis; subalaribus fusco-griseis; pallide griseo marginatis; margine alarum griseo; remigibus subtus fusco-cinereis, margine (interno?) pallide griseo; rostro nigro; pedibus fuscis.

Long. tot. $0^{\mathrm{m}}, 180$; al. $0^{\mathrm{m}}, 103$; caud. $0^{\mathrm{m}}, 088$; rostri $0^{\mathrm{m}}, 020$; tarsi $0^{\mathrm{m}}, 025$.

$H a b$. in Insula Sud-est dicta.

Il De Vis menziona due maschi ed una femmina di questa specie, che a me sembra essere una Pachycephala molto affine, se non identica, colla Pachycephala fortis, Gadow.

(458) Pachycephala schlegeli, Meyer (?).

Pachycephala schlegelii, De Vis, Ann. Rep. Brit. lew Guin. p. 60 (1890) (Musgrave Range). Id., Colon. Papers, p. 111 (1890). - Id., Ibis, 1891, p. 34.

Il De Vis riferisce a questa specie esemplari dei Monti Musgrave, raccolti fra i 7000 ed-i 9000 piedi di altezza; credo che essi dovranno essere confrontati con altri dei Monti Arfak.

(1101) Pachycephala fortis (GaDOW).

Pachycephala fortis, Salvad., Ann. Nus. Civ. Gen. 2) X, p. 830 (Isole Goodenough e Fergusson) (1891).

(186) Hermotimia corinna, SALVAD.

Cinnyris corinna, North, Pr. Linn. Soc. N. S. W. (2) II, p. 444 (nest and eggs, New Britain) (1887).

Hermotimia corinna, Meyer, Ibis, 1890, p. 417 (New Britain, Kubory).

(1103) Hermotimia christianae, Tristr.

Hermotimia christianae, Salvad., Ann. Mus. Civ. Gen. (2) X, p. 380 (Isola Fergusson) (1891).

Per cortesia del Tristram ho potuto esaminare il tipo di questa specie dell'Isola Saint-Aignan; essa somiglia alla $H$. chlorocephala, ma ha la parte anteriore del collo di un bel colore azzurro-acciaio, senza riflessi violetti, ma leggermente verdi; come nota il Tristram, questa specie somiglia molto alla mia H. corinna delle Isole del Duca di York, ma ha lo scudo pettorale azzurro con riflessi più decisamente verdi, il becco più lungo, e tutte le dimensioni un poco maggiori; forse anche la femmina è diversa.

Sono stato sorpreso di trovare nel Museo Britannico un esemplare indicato di Port Moresby e raccolto dal Goldie, simile in tutto al tipo di Saint-Aignan; io sospettu che la provenienza indicata non sia esatta.

(510) Dicaeum aeneum, P. et J.

Dicaeum aeneum, Ramsay, Rec. Austr. Mus. I, p. 5 (1890) (Howla Isl.).

(516) Pristorhamphus versteri, Finsch.

Pristorhamphus versteri, De Vis, Ann. Rep. Rrit. New Guin, p. 60 (1890) (Nount Musgrave).

- Id., Colon. Papers, p. 111 (1890). - Id., Ibis, 1891, p. 34.

[104] 
La presenza di questa specic sul Monte Musgrave, ad 8000 piedi di altezza, come fa rilevare l'editore dell'Ibis (1. c. p. 25) è cosa notevole. Sarebbe importante di confrontare gli esemplari di quella località con altri tipici della Nuova Guinea settentrionale-occidentale.

(521) Myzomela forbesi, Raus.

My zomela forbesi, Salvad, Ann. Mus. Giv. Gen. (2) X, p. 830 (屯, Q , Isola Fergusson) (1891).

La femmina di questa specie somiglia tanto a quella della $M$. nigrita da essere quasi impossibile il distinguerla; tuttavia è un poco più grande ed ba il rosso sbiadito del pileo, dei lati della testa e della gola un po' più vivo.

\section{Sp. 1188 (529bis) Myzomela pulchella, SALVAD. nov. sp.}

Mas. Capite et gula coccineis; corpore reliquo supra fusco-olivaceo; pectore, abdomine et subcaudalibus flavescentibus; pectoris plumis medio fuscis; axillaribus albo-flavidis; remigum pogonio interno, basim versus, albo; caula fusca; rectricum pogonio externo olivaceo limbatis; rostro fusco; pedibus plumbeis.

Long. tot. $0^{\mathrm{m}}, 110$; al. $0^{\mathrm{m}}, 070$; caud. $0^{\mathrm{m}}, 045$; rostri $0^{\mathrm{m}}, 017$; tarsi $0^{\mathrm{m}}, 015$.

Hab. in Nova Hibernia (New Ireland) (Kleinschmidt).

Il tipo è nel Museo Britannico.

Questa specie appartiene al gruppo che comprende la M. boiei, la MI. adolphinae e la $M I$. annabellae, arendo come queste la testa rossa, ma si distingue facilmente per non avere il groppone ed il sopraccoda di color rosso.

(534) Myzomela cineracea, ScLat. (?).

Myzomela cineracea, De Vis, Ann. Rep. Brit. New Guin, p. 60 (1890) (Rossel Island). - Id., Colon. Papers, no. 103, p. 111 (1890). - Id., Ibis, 1891, p. 34.

Io dubito grandemente che la Myzomela dell'Isola Rossel, la più orientale delle Luisiadi, sia diversa dalla $\boldsymbol{M}$. cineracea, Sclat., propria della Nuova Britannia, la quale appartiene ad un altro gruppo d'isole.

Sp. 1189 (550 $\left.0^{\text {his }}\right)$ Melirrhophetes belfordi, DE V1s.

Melirrhophetes belfordi, De Vis, Ann. Rep. Rrit. New Guin. $\uparrow .60$ (1890) (Mount Knutsford): Id., Colon. Papers, no. 103, p. 111 (1890) - Id. Ibis, 1891, p. 34.

Fem. juv. Fronte, sincipite, loris, regione suboculari et auricularibus sordide nigris; fascia supercitiari lata supra oculos incipiente, postice juxta occipitis latera excurrente, alba; regione nuda periophtalmica et temporali, ut videtur, saturate caerulea; palpebra inferiore, ut videtur, rubra; mento et gula grisescente-nigris, fascia alba, a regione mandibulari oriente, circumdata; cervice brunnescentigrisea, regione interscapulari et tectricibus alarum fuscis, margine lato plunarum griseo; supracaudalibus fuscis, vix olivaceo narginatis; corpore subtus collique lateribus griseo-brunneis, abdominis plumis vix griseo-marginatis; tibiis fusco-brunneis; 
subcaudalibus et plumis imis laterum ferrugineis; tectricibus alarum fuscis, majoribus olivaceo-viridi tenuter marginatis; remigibus fuscis, duabus primis exceptis, viridi-aureo marginatis; subalaribus griseo-cinereis; remigibus subtus fusco-cinereis; cauda fusca, rectricibus aureo-viridi tenuiter marginatis et grisescenti-albo terminatis; rostro nigro; pedibus brunneo-rufescentibus; carunculis roseis.

Long. tot. $0^{\mathrm{m}}, 280$; al. $0^{\mathrm{m}}, 160$; caud. $0^{\mathrm{m}}, 145$; rostri culm. $0^{\mathrm{m}}, 033$; tarsi $0^{\mathrm{m}}, 035$.

Hab. in Monte Knutsford, Nova Guinea orientali-meridionali.

Il De Vis ha descritto una femmina giovane; pare che essa si distingua dal II. Teucostephes per non arere la fronte bianca.

(551) Melirrhophetes ochromelas, MEYER.

? Melirrhophetes batesi, Sharpe, Nature, vol. 34, p. 340 (1886).

Melirrhophetes ochromelas, De Vis, Ann. Rep. Brit. New « in. p. 60 (1890)(Hount Knutsford;. - Id., Colon. Papers, no. 103, p. 111 (1890'. - Id., Ibis, 1891, p. 34.

Come ho già detto in altra occasione, è molto probabile che gli esemplari della Nuova Guinea meridionale-orientale ( $M$. batesi, Sharpe) non siano diversi specificamente da quelli della Nuova Guinea settentrionale-occidentale.

(558) Ptilotis analoga, RchB.

Ptilotis analoga, Salvad, Ann. Mus. Giv. Gen. (2) IX, p. 574 (1890) (Rigu).

Io ho fatto notare $(l . c$.$) come un esemplare di Rigo, nella Nuova Guinea meridio-$ nale-orientale, appartenga indubbiamente alla forma colle piume gialle della regione auricolare lunghe e colle piume del groppone molli, copiose, variegate di nero e terminate di bianco all'apice, la quale forma è predominante nella Nuova Guinea settentrionale-occidentale.

Sp. 1190 (558 $8^{\text {bis}) ~ P t i l o t i s ~ a r u e n s i s, ~ S h a r p e . ~}$

Ptilotis analoga, part., Salvad., Orn. Pap. e Mol. II, p. 327 (1880). - Id., Mem. R. Ac. Sc. Tor. (2) XL, p. $250(1890)$.

Ptilotis aruensis, Sharpe, Rep. Alert, p. 19 (1884). - Salvad., Mem. R. Ac. Sc. Tor. (2) XL, p. $251(1890$ )

Ptilotis P. analogae, similis, et eodem modo plumis uropygii mollibus, copiosis, nigro-fusco variegatis, exterioribus albo terminatis, praedita, sed auriculuribus flavis breviusculis distinguenda.

Hab. in Insulis Aru et in Nova Guinea meridionali ad flumen Fly.

Io accennai gì (Orn., II, p. 331) agli esemplari delle località sopra indicate, siccome appartenenti ad una forma distinta.

Sp. 1191 (558ter) Ptilotis gracilis, Gould.

Ptilotis gracilis, Gould, P. Z. S. 1866, p. 227. - Ramsay, Pr. Linn. Soc. N. S. W. II, p. 189, no. 315; p. 207 (1877). - Sharpe, Rep. Alert, p. 19 (1884). - Ramsay, Tab. List, p. 12 (1888). Salvad., Mem. R. Ac. Sc. Tor. (2) XL, p. 251 (1890), - Id., Ann. Mus. (Siv. Gen. (2) IX, p. 574 (1890).

Meliphaga gracilis, Gieh, Ther, 0rn. II, p. 557 (1875).

[106] 
Ptilotis analoga, part., Salval., Orn. Pap. g Mol. II, n. 327 (1881). - Id., Mem. R. Ac. Sc. Tor. (2) XL, p. 250 (1890).

Ptilotis Pt. analogae similis, sed plumis uropygii unicoloribus, haud nigro variegatis, neque albo terminatis, et plumis auricularibus breviusculis distinguenda.

Hab. in Nova Guinea, praesertim in parte meridionali-orientali, et in Nova Hollandia settentrionali ad Caput York.

Questa forma predomina nella Nuova Guinea meridionale-orientale, ma s'incontra anche nella Nuova Guinea settentrionale-occidentale ed è la sola che si trovi nell'Isola di Mliosnom nella Baia del Geelvink.

(563) I tilotis sonoroides, G. R. Gr.

Ptilotis sonoroides, Salvad., Ann. Mus. Civ. Gen. (2) X, p. 817 (Isola Killertonj (1891).

(564) Ptilotis germana, RAMSAY.

Prilotis germana, Salvad., Ann. Mus. Civ. Gen. (2) IX, p. 576 (1890) (Port Moresby, Loria)

(565) Ptilotis subfrenata, SALVAD.

Ptilotis subfrenata, De Vis, Ann. Rep. Brito. New Guin., p. 60 (1890) (Mlusgrave Rango). - Id., Culon. Papers, no. 103, p. 112 (1890). - Id., Ibis, 1891, p. 35.

\section{(566) Ptilotis erythropleura, SALVAD.}

Ptilotis erytiropleura, De Vis, II. cc. (1890-91) (Musgrave Range).

Questa e la precedente specie non erano state trovate finora nella Nuova Guinea orientale-meridionale.

(569) Pycnopygius stictocephalus, SALvaD.

Pycnopygius stictocephalus, Salvad., Ann. Mus. Liv. Gen. (2) IX, p. 577 (1890) (Rigo).

(585) Philemon cockerelli, Scrat.

Philemon cockerelli, North, Pr. Linn. Suc. N. S. W. (2) II, p. 44 '́t (eggs, New Britain) (1887).

(587) Tropidorhynchus novae-guineae, S. MïLL.

Tropidorhynchus novae-guineae, De Vis, 11. cc. (1890, 1891) (Tormanby Island).

Sp. 1192 (606 $\left.6^{\text {bis }}\right)$ Zosterops pallidipes, DE VIs.

Zosterops pallidipes, De Vis, Ann. Rep. Brit. New Guin. p. 60 (1890). - Id., Colon. Papers, no. 103, p. 112 (1890). - Id, Ibis, 1891, p. 35.

Mas. Simitis Z. branneicandae, Salvad., sed corpore subtus minus flavo et magis flavo-virescente; pedibus pallide carneis; mandibulae et maxillae tomiis apicem versus flavicanti-albis.

$H a b$. in Insula Rossel.

(619) Pitta mackloti, Tem.

Pitta novae-hiberniae, North, Pr. Linn. Soc. N. S. W. (2. II, p. 443 (eggs, Duke of York group) (1887) 
Pitta rubrinucha, De Vis (nec Wall.), Ann. Rep. Brit. New (iuin. p. 60 (1890) (Exton Junction, 1200 feet). - Id., Colon. Papers, no. 103, p. 112 (1890). - Id., Ibis, 1891, p. 35.

Pitta mackloti, Salvad., Ann. Mlus. Giv. Gen. (2) X, p. 818 (Bujakori) (1891).

La $P$. rubrinucha, Wall. è confinata nell'Isola di Buru, e quindi è impossibile che alla medesima si possano riferire gli esemplari di Exton Junction, i quali con ogni probabilità appartengono alla $P$. mackloti, Temm., od alla $P$. finschi, Ramsay, ammesso che questa sia una specie distinta.

Sp. 1193 (619 ${ }^{\text {bis }}$ ) Pitta loriae, SaLvad.

Pitta loriae, Salvad., Ann. Mus. 'iv. Gen. (2) IX, p. 579 (1890).

Pitta P. mackloti simillima, sed cervice fusco-rubra capite concolore, haud laetiore vel clariore quam capite, distinguenda.

$\mathrm{Hab}$. in Insula Su-a-u, ad Caput meridionale (South Cape) Novae Guineae.

(682) Eupetes leucostictus, ScLat.

Eupetes leucostictus, De Vis, Ann. Rep. Brit. New Guin. p. 60 (1890). - Id., Colon. Papers, no. 103, p. 112 (1890). - Id., Ibis, 1891, p. 35.

Questa specie non si conosceva finora della Nuova Guinea meridionale-orientale; il De Vis non menziona il luogo preciso ove essa è stata trovata.

GeN. MERULA, LeAch.

Merula, Leach, Cat. Brit. Mus. p. 20 (1816).

Cychloides, Kaup, Natürl. Syst, p. 153 (1829)

Copsychus, Kaup, Natürl. Syst. p. 157 (1829)

Thoracocincla, Rehb., Nat. Syst. t. XLIII (1850)

Cichloselys, Bp., Compt. Rend. XXXVIII, p. 5 (1854).
Typus :

Turdus merula. L.

Turdus alrigularis, Temm.

Turdus torqualus, I.

Turdus torquritus, L.

Turdus cardis, Temm.

Sp. 1194 (1015) Merula papuensis, DE Vis.

Merula papuensis, De Vis, Ann. Rap. Brit. New Guin. p. 60 (1890). - Id., Colon. Papers, no. 103, p. 112 (1890). - Id., Ibis, 1891, p. 35.

Supra fusco-brunnea, capite pallidiore; mento et gula pallide grisescentebrunneis, pectore et abdomine brunneis obscurioribus, sed notaeo pallidioribus; plumis ante regionem analem albo terminatis; subcaudalium scapis pallidis, apicibus obsolete pallide rufo-brunneis; tibiis pallide brunneis; pedibus flavis; rostro flavo.

Long. tot. $0^{\mathrm{m}}, 350$; al. $0^{\mathrm{m}}, 135$; caud. $0^{\mathrm{m}}, 110$; rostri $0^{\mathrm{m}}, 020$; tarsi $0^{\mathrm{m}}, 035$; remigis primae $0^{\mathrm{m}}, 025$.

$H a b$, in Monte Victoria, Nova Guinea meridionali-orientali.

Il De Vis descrive un secondo esemplare, apparentemente non al tutto adulto, col colore grigio scuro della gola, che gradatamente si unisce con quello bruno del petto, con tutte le piume di color più chiaro e meno vivace, e colle macchie sul sottocoda e sulle piume preanali più distinte. Becco arancio; piedi gialli; prima remigante lunga 15 millimetri. 
(650) IIunia molucca (Linn.).

Uroloncha molucca, Sharpe, Ciat. B. XIII, p. 367 (1890).

Lo Sharpe ha separato specificamente gli esemplari di Flores e di Celebes (Uroloncha propinqua, Sharpe).

\section{Sp. 1195 (650'is) Munia nisoria (TEMro).}

? Gros-bec tacheté de Java, Briss., Urn. 1II, p. 239, nl. XIII, f. $2(1760)$.

? Loxia puntulata, part., Linn., S. X. J, p. 302, no. 18 (1766).

? Loxia undulata, Lath. (nec Müll.), Ind. Orn. I, p. 387 (1790).

Fringilla punctularia, IIors. (nec Gm.; Tr. Linn. Soc. XIII, p. 161 (1820) (Java).

Fringilla nisoria, Temm., Pl. Col. III, pl. 500, f. 2 (1830) (Java)., - Vald., lbis, 1869, p. 211.

Oxycerca nisoria, G. B. Gr., App. to a List of Gen. B. p. 10 (1842).

A madina punctularia, Hay (nec Gm.), J. A. S. B. XIV, p. 554 (nota) (1845). - G. R. Gir., Gen. B. II, p. 370, nо. 35 (part.) (1849).

Nunia punctularia, Blyth (nec Gm.\%, Clat. B. Mus. A. S. B. p. 117, no. 625 (1849). - Bp., Consp. I, p. 452 (part.) (1850\% - Horsf, and Noore, Cat. B. Nus. E. I. Comp. II, p. 505 (syn. emend.) (1856). - Cantor, P. Z. S. 1859, p. 443 (Penang). - Wall., P. Z. S. 1863, p. 486 (Lombock, Flores, Timor). - Salvad, Ann. Nus. Civ. Gen. XIV, p. 237 (1879) (Sumatra). - Nichols., Ibis, 1883, p. 254 (Sumatra). - ? Büttik, Jot. Leyd. Mlus. IX, p. 71 (1886) (Padang, Sumatra). - Vorderm., Nat. Tijdschr. Ned. Ind. XLIX, p. 410, no. 383 (1889) (Sumatra).

Uroloncha punctularia, Kichb. (nec Gm.', Sing、ög. p. 45, t. XVI. f. 141, 142 (1863!.

Lonchura undulata, G. R. Gr., Hand List, II, p. 56, no. 6778 (1870) (Java). - Vorderm., Nat. Tijdschr. Ned. Ind. XLIV, p. 224 (1885) (Java).

Munia nisoria, Blyth, Ibis, 1870, p. 172 (Nalayan race). - ? Wald., Tr. Zool. Soc. VIII, p. 73 (1872) (Gelebes). - Salvad., Ann. Mlus. Civ. Gen. (2) IV, p. 552 (1887) (Nias). - Sharpe, Cat. B. XIII, p. $352(1890)$.

Amadina nisoria, Hume, Str. Feath. VIII, p. 66 (1879) (Penang, Malacca, Singapore). - Oat. Cat., B. of Brit. Burm. I, p. 358 (1883).

Munia molucca, Sclat. (nec Linn.), P. Z. S. 1883, pp. 5', 195 (Timorlaut)。 - Forb., P. Z. S. 1884, p. 433 (Tenimber Islands). - Neyer, Zeitschr. f. ges. Orn. I, p. 194 (Timorlaut). - Salvad., Mem. R. Ac. Sc. Tor. (2) XXXIX, p. 265 (part.) (1890).

Supra castanea, Tineis scapalibus tenuissimis albis notata; genis et gula saturate castaneis; uropygio, supracaudalibus et cauda cinereis, plumarum marginibus albido-mellinis; uropygii plumis lunulis albidis notatis; corpore subtus albido; pectore brunneo-lunulato; lateribus nigro-lumulatis et transfasciatis; abdomine imo et subcaudalibus albidis; his lumilis fuscis obtectis notatis; alis castaneis, dorso concoloribus; remigibus intus fuscis, rufescente marginatis; subalaribus rufescentibus; " iride rubra, rostro supra nigro, subtus cinereo; pedibus cinereis 》 Modigliani).

Long. tot. $0^{\mathrm{m}}, 110$; al. $0^{\mathrm{m}}, 052$; caud. $0^{\mathrm{m}}, 043$; rostri $0^{\mathrm{m}}, 012$; tarsi $0^{\mathrm{m}}, 015$.

Foemina. Mari simitis.

Juv. Supra brumea, fere unicolor; subtus albido-rufescens.

Hab. Penang (Cantor); Malacca (Wallace, Davison); Singapore (Davisoni); Sumatra (Beccari, Bock, MLodigliani); Nias (Modigliani); Java (Horsfield, Forbes); Lombock (Wallace); Flores (Wallace); Timor (Wallace); Celebes (Wallace); Timorlaut (Forbes).

Questa specie somiglia molto alla Mr. punctulata (L.) dell'India, dalla quale si 
distingue pel colore grigio del groppone, del sopraccoda e della coda, le quali parti in quella specie sono di color bruno fulvo dorato. Secondo lo Sharpe, gli esemplari di Flores hanno la coda più giallognola, e, secondo Lord Walden, quelli di Celebes, od almeno l'unico da lui esaminato, aveva le piume del sopraccoda ed i margini delle timoniere di color verde-oliva.

La $M$. nisoria occupa un'area molto estesa; essa è stata trovata nelle Isole Tenimber, o Timorlaut dal Forbes, ma per errore gli esemplari di queste isole furono attribuite alla II. nolucca dallo Sclater, laddove, secondo lo Sharpe, essi spettano alla specie presente.

E cosa singolare che to Sharpe, nella sinonimia della medesima, abbia ommesso tutte le citazioni che si riferiscono a Sumatra, Lombock, Flores, Timor, Celebes e Timorlaut! Inoltre la citazione relativa al nome Oxycerca nisoria è sbagliata e deve essere rettificata nel modo sopra indicato.

\section{(651) Munia tristissima, WALL}

Uroloncha tristissima, Sharpe, Cat. B. XIII, p. 365 (1890).

Miunia tristissima, Salvad., Ann. Nus. Civ. Gen. (2), X, p. 818 (Nuova Guinea orientale-meridio. nale) (1891).

(652) Munia leucosticta, SAlvad.

Uroloncha leucosticta; Sharpe, Cat. B. XIII, p. 365 (1890).

(653) Munia jagori, $\mathrm{C}_{\Delta \mathrm{B} \text {. }}$

Munia jagori, Sharpe, Cat. B. XIII, p. 337 (1890).

Lo Sharpe non menziona Halmahera fra i luoghi abitati da questa specie, che egli vuole confinata nelle Isole Filippine.

(654) Munia forbesi, Sclat.

Munia forbesi, Sharpe, Cat. B. XIIf, p. 343 (1890).

(65) Munia melaena, Sclat.

Munia melaena, North, Pr. Linn. Soc. ‥ S. W. (2) II, p. 443 (eggs, New Britain) (1887). - Sharpe, Cat. B. XIII, p. $343(1890)$.

(1016) Munia grandis, SHARPE.

Munia grandis, Sharpe, Cat. B. XIII, p. 344 (1890). - Salrad., Ann. Mus. Giv. Gen. (2), X, p. 818 (Kunirira) (1891).

(656) Donacicola caniceps (SALVAD.).

Munia caniceps, Sharpe, Cat. B. XIII, p. 345 (1890).

(1128) Donacicola hunsteini, FinscI.

Munia hunsteini, Sharpe, Cat. B. XIII, p. 344 (1890).

(657) Donacicola spectabilis, Sclat.

Munia spectabilis, Sharpe, (Yat. B. XIII, p. 342 (1890).

Donacicola spectabilis, Meyer, Ibis, 1890, p. 417.

[110] 
(658) Donacicola nigriceps, Ramsay.

MHunia nigriceps, Sharpe, Cat. B. XIII, p. 341 (1890).

Donacicola nigriceps, Salvad., Ann. Mus. Uiv. Gen. (2) X, p. 819 (Isola Killerton) (1891).

(659) Erythrura trichroa (KiTru.).

Erythrura trichroa, Sharpe, Cat. B. XIII, p. 385 (1890).

(1129) Erythrura forbesi, SHARPE.

Erythrura tricolor, Sclat. (nec Vieill.), P. Z. S. 1883, pp. 51, 20. - Salvad., Mern. H. Ac. Sc. Tor. (2) XI, p. 267 (1890).

Erythrura trichroa, Forbes (nec KittI.), P. Z. S. 1884, p. 443. - IU., Nat. Wand. lialay. Archip. p. 365 (1885).

Erythrura forbesi, Sharpe, Cat. B. XIII, p. 387 (1890)

Similis E. tricolori, sed occipite, cervice, dorso et alis ommino viridibus, minime caeruleo finctis.

Hab. Ins. Tenimberensibus.

Ho tratto la frase diagnostica dalla breve descrizione dello Sharpe, il quale ha distinto la forma delle Isole Tenimber da quella di 'Timor.

Questa specie tiene il posto della $E$. tricolor, da me annoverata nelle Aggiunte $(l . c$.$) .$

\section{(661) Neochmia phaeton (H. et J.).}

Neochmia phaeton, Sharpe, Cat. B. XIII, p. 389 (1890).

Lo Sharpe ha ommesso di annoverare la Nuova Guinea tra i luoghi abitati da questa specie, e quindi anche le citazioni che a quella località si riferiscono.

(662) Sturnia violacea (Bopd.).

Sturnia violacea, Sharpe, Cat. B. XIII, p. 70 (1890).

(663) Calornis metallica (ТЕมณ.).

Calornis metallica, var. nitida, North, Pr. Linn. Soc. N. S. W. II, p. 443 (eggs, Duke of York Island) (1887).

Calornis metallica, Sharpe, Cat. B. XIII, p. 138 (1890). - Salvad., Ann. Mus. Civ. Gen. (2) IX, p. 580 (1890) (Rigo).

Galornis nitida, Neyer, Ibis, 1890, p. 417 (New Britain).

Il Neyer sostiene che gli esemplari dell'Arcipelago di Bismarck (Ca7ornis nitida, G. R. Gr.) mancano sempre della macchia verde metallica nel mezzo dell'area interscapolare violetta, e che perciò essi debbono essere considerati come specificamente distinti.

(664) Calornis purpureiceps, Salvad.

Galornis purpureiceps, Sharpe, Cat. B. XIII, p. 142 (1890).

(665) Calornis inornata, SALVAD.

Calornis inornata, Sharpe, Cat. B. XIII, p. 141 (1890). 
(666) Galornis fuscovirescens, SaLvaD.

Calornis fuscovirescens, Sharpe, Cat. B. XIII, p. 141 (1890).

(1130) Calornis circumscripta, MEYER.

Calornis gularis, Sharpe, Cat. B. XIII, p. 141 (1890).

Io ho sostenuto (Ibis, 1884, p. 455; P. Z. S. 1884, p. 579; Mem. R. Ac. Se. Tor. (2) XL, p. 267): $1^{\circ}$ che gli esemplari di Timorlaut appartengono ad una specie distinta da tutte le altre; $2^{\circ}$ che essi non potevano essere riferiti alla $C$. gularis, Gr., descritta sopra un individuo di Mrysol.

Io fondava queste mie considerazioni sulla memoria dell'esame fatto nel 1877 del tipo della C. gularis, Gr. conservato nel Museo Britannico; quell'esemplare, « colla gola cospicuamente porporina », mi era parso « simile in tutto ad altri esemplari di Halmahera e del Capo York della C. metallica».

Dopo aver riesaminato recentemente nel Mruseo Britannico il tipo della C. gutaris, io mi trovo imbarazzato nell'esprimere la mia opinione intorno al medesimo; quell'esemplare non corrisponde più all'idea che io me ne ero fatto, e confesso di non essere capace di darne una soddisfacente spiegazione. Dobbiamo credere che quell'esemplare spetti in realtà alla $C$. metallica e che, nella variabilità di questa specie, ci presenti un caso di variazione individuale, simile ad una forma che è diventata costante nelle Isole di Timor-laut? Comunque sia, io non posso indurmi a credere che esso sia un esemplare della forma propria di queste isole tanto lontane da Mysol.

(667) Calornis obscura (Forsten).

Calornis obscura, Sharpe, Cat. B. XIII, p. 149 (1890).

(668) Galornis cantoroides, G. R. GR.

Aplonis cantoroides, Sharpe, Cat. B. XIII, p 128 (1890).

Calornis cantoroides, Salvad., Ann. Mus. Civ. Gen. (2) IX, p. 581 (1890); X, p. 819 (1891).

(1131) Galornis crassa, Sciar.

Aplonis crassa, Sharpe, Cat. B. XIII, p. 134 (1890).

(1017) Calornis feadensis, Solat.

Aplonis feadensis, Sharpe, Cat. B. XIII, p. 129 (1890).

(669) Macruropsar magnus (Rosens.).

Macruropsar magnus, Sharpe, Cat. B. XIII, p. 152 (1890).

(670) Lamprocorax grandis, SALVAD.

Calornis grandis, Sharpe, Cat. B. XIII, p. 150 (1890).

(1018) Lamprocorax minor (RAMSAT).

Calornis minor, Sharpe (nec S. Müll.), Cat. B. XIII, p. 151 (nec p. 142) (1890).

Lo Sharpe, il quale, secondo me, a torto ha voluto riunire questa e la prece[112] 
dente specie nel genere Calornis, ha commesso l'inarvertenza di chiamare la prima $C$. minor, mentre usa lo stesso nome per un'altra specie dello stesso genere, propria di Timor.

(671) Basileornis corythaix, WALL.

Basileornis corythaix, Sharpe; Cat. B. XIII, p. 93 i1890;

(672) Melanopyrrhus anais (Less.).

Melanopyrrhus anais, Sharpe, Cat. B. XIII, p. 113 (1890).

(673) Melanopyrrhus orientalis (ScHLEG.).

Melanopyrrhus orientalis, Sharpe, Cat. B. XIII, p. 114 (1890).

(674) Mino dumonti, Less.

Mino dumonti, Sharpe, Cat. B. XIII, p. 111 (1890) - Salvad, Ann. Mis. Civ. Gen. (2) IX, p. 581 (1890) - Meyer, Ibis, 1890, p. 418. - Salvad., op. cit X, p. 819 (1891).

(675) Mino kreffti, Sclat.

Mino kreffti, Sharpe, Cat. B. XIII, p. 112 (1890).

(688) Corvus orru, S. MüLL.

Corvus orru, Salvad., Ann. Mus. Giv. Gen. (2) X, p. 831 (Isola Fergusson) (1891).

(684) Corvus salvadorii, Finsch.

Corvus salvadorii, Salvad., Ann. Mus. Civ. Gen. (2) IX, p. 583 (1890) (Rigo).

(692) Manucodia comriei, ScLıt.

Manucodia comriei, Salvad., Ann. Mus. Civ. Gen. (2) X, p. 831 (Isola Goddenough ed Isola Fergusson) (1891).

(693) Manucodia chalybeata (Penn.).

Manucodia chalybeata, Salvad., Ann. Mus. Civ. Gen. (2) X, p. 820 (Nuova Guinea or.) (1891).

(1136) Phonygama purpureoviolacea, Merer.

Phonygama purpureoviolaceus, De Vis, Ann. Rep. Brit. New Guin.p. 60 (1890) (Goodwin Spur, 5000 feet). - Id., Colon. Papers, no. 103, p. 112 (1890; - Id, Ibis, 1891, p. 36.

(1019) Phonygama hunsteini, SHARPE.

Phonygama hunsteini, De Vis, Ann. Hep. Brit. New Guin. p. 60 (1890). - Id, Colon. Papers, no. 103, p. 112. - Id., Ibis, 1891, p. 36.

Il De Vis, menzionando questa specie, non indica il luogo in cui fu raccolta, la quale cosa sarebbe stata importante, giacchè se nell'Isola Normanby, come sospetto, sarebbe avvalorato il mio dubbio della sua identita colla $\boldsymbol{M}$. thomsoni.

(1187) Phonygama thomsoni, TRISTR.

Phonygama thomsoni, Salvad., Ann. Mus. Giv. Gen. (2) X, p. 832 (Isola Goddenough) (1891).

Forse non diversa dalla Phonygama hunsteini, Sharpe.

10 T. Salvadori. 
(1188) Parotia lawesi, Ramsay.

Parotia sexpennis, De Vis (nec Bodd.', Ann. Rep. Brit. New Guin. p. 60 (1890). - Ido, Colon. Papers, no. 103, p. 113 (1890). - Id., Ibis, 1891, p. 36.

Parotia lawesi, Goodw., Ibis, 1890, p. 151.

Gii esemplari che il De Vis attribuisce alla $P$. sexpennis furono raccolti sul Monte Belford fra i 3600 ed i 7000 piedi ; mi sembra molto probabile che $\dot{\mathbf{I}}$ medesimi siano da riferirsi alla $P$. lawesi, che è la forma propria della Nuova Guinea meridionale-orientale.

(1139) Lophorhina minor, RAMsur.

Lophorhina superba, De Vis (nec Penn.), Ann. Rep. Brit. NewGuin. p. 60 (1890) (Goodwin Spur, 5000 to 7000 feet). - Id., Colon. Papers, nı. 103, p. 113 (1890). - Id., Ibis, 189.

Lophorhina minor, Goodw., Ibis, 1890, p. 152.

(1140) Astrarchia stephaniae (Finscr).

Astrarchia stephaniae, Goodw., P. Z. S. 1889, p. 451. - Id., Ibis, 1890, p. 153. - De Vis, Ann. Rep. Brit. New Guir. p. 61 (1890) (Mount Knutsford, 8000 feet). - Id., Colon. Papers, no. 103, p. 113. - Id., Ibis, 1891, p. 36.

(1141, 1142) Epimachus meyeri, Finsch.

Epimachus macleayanae, Goodw., P. Z. S. 1889, p. 451. - Id., Ibis, 1890, p. 152.

Epimachus meyeri, De Yis, Ann. Hep. Brit. New Guin. p. 60 (1890) (Musgrave Range, 6000 to 7000 feet). - Id., Colon. Papers, no. 103, p. 113 (1890). - Id., Ibis, 1891, p. 36.

Non credo possibile di conservare più alcun dubbio intorno alla identità specifica dell'E. macteayae (ठ) coll' $E$. meyeri.

(702) Epimachus ellioti, WARD.

Epimachus ellioti, Meyer, Ibis, 1890, p. 418.

Il Meyer menziona e descrive un esemplare maschio completo di questa specie, della quale se ne conosceva finora soltanto uno incompleto.

« Becco $0^{\mathrm{m}}, 041$; ala $0^{\mathrm{m}}, 202$; coda $0^{\mathrm{m}}, 403$; tarso $0^{\mathrm{m}}, 050 »$ (Meyer).

(1143) Drepanornis cervinicauda, ScLAt.

Drepanornis cervinicauda, Goodw., Ibis, 1890, p. 152. - Salvad., Ann. Mus. Civ. Gen. (2) IX, p. 585 (1890).

(704) Drepanornis bruijni, Odst.

Drepanornis bruijni, Meyer, Ibis, 1890, p. 419 (Walckenaers Bay, West of llumboldt Bay).

Il Meyer fa notare come il maschio descritto dall' Oustalet non sia al tutto adulto, e menziona alcune differenze fra la descrizione del medesimo ed un altro esemplare in abito perfetto posseduto dal Museo di Dresda.

\section{(1020) Craspedophora intercedens (SHARPE).}

Ptilorhis magnifica, Goodw. (nec Vieill.), Ibis, 1890, p. 151.- De Vis, Ann. Rep. Brit. New Guin. p. 61 (1890) (Mount Kowald, 2500 feet). - Id., Colon. Papers, no. 103, p. 113 (1890! - Id., Ibis, 1801, p. 37.

Graspedophora intercedens, Meyer, Ibis, 1890, p. 419 (Constantine Harbour). - Salvad., Ann. Mus. Civ. Gen. (2) X, p. 821 (1891).

[114] 
GEN. PARYPHEPHORUS, MEYER.

Type:

Paryphephorus, Meyer, lbis, 1890, p. 420

Craspedophora duivenbodiei, Mejer.

Sp. 1196 (705' $\left.{ }^{\text {เr}}\right)$ Paryphephorus duivenbodei (MEYER).

Craspedophora duivenbodei, Neyer, Ibis, 1890, p. 419, pl. XII.

Nigro-velutinus, purpureo nitens; pileo, scuto gutturali, rectricibusque duabus mediis nitidissime viridibus, scuto gutturali inferius margine cyanescente notato; pectore summo nitide oleagineo; lateribus capitis purpureo nitentibus; cervice pallio nigro velutino, purpureo nitente, ornata; rostro nigro; pedibus fuscis.

« Long. tot. $0^{\mathrm{m}}, 280$; al. $0^{\mathrm{m}}, 160$; caud. $0^{\mathrm{m}}, 109$; rostri culm. $0^{\mathrm{m}}, 040$; tarsi $0^{m \prime \prime}, 033 \gg$ (ILeyer).

Hab. in Nova Guinea (?).

Ho visto in Londra il tipo di questa specie, che giustamente il Meyer ha proposto di separare genericamente dalle Craspedophorae; a me sembra che il nuovo genere sia intermedio al genere Ptilorhis ed al genere Lophorina, cui somiglia pel pallio cervicale, sebbene questo sia di forma circolare, e molto più piccolo. Le piume mediane del pallio sono più brevi delle laterali, la quale cosa non appare nella figura.

S'ignora la esatta provenienza dell'esemplare tipico.

\section{(706) Seleucides nigricans (Sнат).}

Seleucides niger, Goodw., Ibis, 1890, p. 150 (S. E. New Guinea).

Il Goodwin menziona questa specie, di cui dice di aver udito il grido, ma di non averne ottenuto alcun esemplare.

(1144) Paradisea finschi, Meren,

Paradisea finschi, Meyer, Ibis, 1890, p. 420.

Il Meyer ci fa sapere di aver ricevuto altri sei esemplari di questa specie dalla Baia di Costantino e dalle sue vicinanze (Weisser e Kubary), e secondo le sue ultime asserzioni, le differenze prima affermate rispetto al colorito, in confronto colla $P$. minor, non sono reali; le differenze si ridurrebbero quindi alle dimensioni un poco minori, le lunghe piume dei fianchi essendo un poco più corte ed il becco più sottile; il giallo sulle piccole cuopritici delle ali sarebbe meno esteso e la regione interscapolare un poco più scura.

(1146) Paradisea augustae-victoriae, $\mathrm{O}_{A B}$.

Paradisea augustae victoriae, Meyer, Ibis, 1890, p. 421.

Il Meyer menziona un maschio ricevuto dalla Kaiserwilhelmsland, ma senza precisa indicazione di luogo, quindi la esatta patria di questa specie non si conosce ancora. 
(711) Paradisea raggiana, Sclat.

Paradisea raggiana, Goodw., Ibis, 1890, p. 151. - Salval., Ann. Mus. Civ. Gen. (2) IX, p. 558 (1890). - De Vis, Ann. Rep. Brit. Nerv Guin. r. 61 (1890) (3000 feet). - Id., (Colon. Papers, no. 103, p. 113 (1890) - Id., Ibis, 1891, p. 37. - Salvad., Ann. Mus. Civ. Gen. (2) X, p. 821 (1891).

(1147) Paradisea decora, Saly, et GodM.

Paradisea decora, De Vis, Ann. Rep. Brit. Nerw Guin. p. 61 (1890) (Fergusson Island unly). - Id., Colon. Papers, no. 103, p. 113 1890). - Id., Ibis, 1891, p. 37. - Salvad., Ann. Mus. Civ. Gen. (2) X, p. 833 (Isola Fergusson' (1891:

\section{(1149) Diphyllodes hunsteini, MEYer.}

Diphyllodes chrysoptera, Goodw. (nec Gould ?), Ibis, 1890, p. 153 (Knutsford Range).

Diphyllodes magnifica, De Vis (nec Penn.), Ann. Rep. Brit. New Guin. p. 61 (1890) (Mount Knutsford, 3000 to 4000 feet). - Id., Colon. Papers, no. 103, p. 113 (1890). - Id., Ibis, 1891, p. 37.

Non vi può essere dubbio che il De Vis ha errato nel riferire gli esemplari del genere Diphyllodes del Monte Knutsford alla D. magnifica, che è la forma settentrionale-occidentale, la quale nella Nuova Guinea meridionale-orientale è rappresentata dalla $D$. hunsteini sui Monti Owen-Stanley e dalla $D$. chrysoptera più al basso presso Choqueri (Goldie), se pure sono queste due specie veramente distinte.

\section{GEN. GNEMOPHILUS, DE Vis.}

Typus:

Cnemophilus, De Vis, Ann. Rep. Brit. New. Guin. p. 61 (1890) - Chemophilus macgreyorii, De V'is.

\section{Sp. 1197 (716 ${ }^{\text {bis }}$ ) Gnemophilus macgregorii, De VIS.}

Xanthomelus macgregori, Goodw., Ibis, 1890, p. 153 (descr. incompleta).

Cnemophilus macgregorii, De Vis, Ann. Rep. Brit. Nerw Gnin. p. 61 (1890). - Id., Colon. Papers, no. 103, p. 115 (1890). - Id., Ibis, 1891, p. 40 (1).

Plumis frontalibus et nasalilus elongatis, cristam fronto-nasalem formantibus; crista frontali compressa retrorsum versa, e quatuor plumis arcuatis occipitem attingentibus, aureo-brumea; pilei plumis in medio bipartitis, sulcum formantibus, in quo crista recepta est; parte ima cristae fronto-nasalis castaneo-nigra, parte superiore ejusdem, fronte et regione supraorbitati 7aete mbro aurantiacis; capite (supra), collo (postico) et dorso summo aure-flavis, sericeis; dorso imo, uropygio et supracauda7ibus flavo-brunnescentibus; alis et cauda cimnamomeo-brumeis, remigum marginibus pallidioribus; subalaribus pallide brunneis, nigro variis; corpore subtus, genis, fascia stricta supraoculari, auricularibus et tibiis nigris; pedibus et rostro saturate brunneis, hoc in apice pallidiore.

Long. al. $0^{\mathrm{m}}, 100$; rostri $0^{\mathrm{m}}, 018$; tarsi $0^{\mathrm{m}}, 037$.

$H a b$. in Monte Knutsford, Nova Guinea Orientali (MacGrogor).

Un solo esemplare maschio, colla coda imperfetta, perchè rovinata dal colpo, fu

(1) Durante la stampa del presente lavoro, lo Sclater ha pubblicato una breve nota intorno is questo uccello, accompagnandola con una figura del medesimo: « Remarks on Macgregor's Paradisebird », Cnemophilus macgregorii (Ibis, 1891, pp. 414, 415, p]. X).

$[116]$ 
raccolto durante la spedizione del MacGregor all'altezza di 11,000 piedi. Io ho visto detto esemplare in Londra, presso lo Sclater. Questa specie è notevolissima, oltrechè pel colorito, per la conformazione del ciuffo frontale, il quale nel riposo si alloga nel solco mediano del pileo; essa merita veramente di formare il tipo di un genere distinto, che, secondo me, è più affine al genere Diphyllodes, che non ai generi Amblyornis - Xanthomelus, come vorrebbero il Goodwin ed il De Vis.

\section{(717) Cicinnurus regius (Lisn.).}

Cicinnurus regius, Godw., - Ibis, 1890, p. 151. - Meyer, Ibis, 1890, p. 421. - De Vis, Ann. Rep. Brit. New Guin. p. 61 (1890) (Passim, to 2000 feet). - Id., Colon. Papers, no. 103, p. 113 (1890. - Id., Ibis, 1891, p. 37. - Salvad., Ann. IIus. Civ. Gen. (2) X, p. 822 (1891).

\section{(1151) Amblyornis subalaris, SHarpe.}

Amblyornis subalaris, Goodw., P. Z. S. 1889, p. 451. - Id., Ibis, 1890, p. 155. - De Vis, Ann. Rep. Brit. New Guin. p. 61 (1890) (Nusgrave Range, 6000 to 9000 feet). - Id., Colon. Papers, no. 103, p. 113 (1890). - Id,, Ibis, 1891, p. 37.

Il Goodwin descrive e figura il luogo di sollazzo di questo uccello, formatö di una costruzione a volta; fatta di ramoscelli curvati, dalla quale sporgono i rami di un arboscello; essa è aperta anteriormente e lascia vedere una costruzione circolare interna fatta di muschi ed ornata con fiori e semi, nel mezzo della quale sorge il fusto dell'alberetto, cui sono addossati numerosi ramoscelli.

\section{Sp. 1198 (721 ${ }^{\text {ter }}$ ) Amblyornis musgraviana, Goodw.}

Amblyornis musgravii, Goodw., P. Z. S. 1889 , p. 451.

Amblyornis musgravianus, Goodw., Ibis, 1890, p. 153.

Amblyornis macgregoriae, De Vis, Ann. Rep. Brit. New Guin, p. 61 (1890). - Id., Colon. Papers, no. 103, p. 113 (1890). - Id., Ibis, 1891, p. 37. - Salvad., Ann. Mus. Giv. Gen. (2) X, p. $822(1891)$

Mas. Amblyornis A. subalari paullo major, alis et dorso sordide et obscure viridibus; guta, pectore abdomincque brunneis; subalaribus flavescente-brunneis; crista aurantiaco-auren, obsolete fusco striolata, plumis fusco terminatis; iride brumnea; rostri basi cornea; pedibus nigris (ex Goodwin).

« Long. tot. $0^{\mathrm{m}}, 245-0^{\mathrm{m}}, 255$; al. $0^{\mathrm{m}}, 142-0^{\mathrm{m}}, 148$; rostri culm. $0^{\mathrm{m}}, 020$; tarsi $0^{\mathrm{m}}, 037$ (De Vis).

Foem. Supra olivaceo-brunnea, dorso, tectricibus alarum, capite et cervice lacviter fulvescentibus, plumis capitis elongatis, cristam copiosam, cervicem attingentem; formantibus; alis et cauda ut in mare; Toris, Tateribus capitis et auricularibus brunneis, striis scapatibus pallidioribus; corpore subtus pallide rufo-brunneo, otivaceo tincto; axillaribus et subalaribus pallide isabellinis, uti in mare $\mathbf{A}$. subalaris; remigibus subtus cinereo-fuscis in pogonio interno, basin versus sordide flavicante marginatis; gula striis scapalibus pallidis notata; tibiis fuscis; cauda. supra brunnea, subtus sordide flavo tincta; pedibus nigris; maxilla fusca, mandibula pallida (ex De Vis).

Hab. in Montibus Musgrave, Nova Guinea orientali. 
Cinque esemplari di questa specie, due maschi e tre femmine, furono raccolti durante la spedizione del MacGregor, fra i 7000 ed i 9000 piedi di altezza.

Io non riesco ad afferrare i caratteri che distinguono questa specie dall' $A$. subalaris, Sharpe.

Il pergolato, o luogo di sollazzo di questo uccello è stato descritto dal Goodwin. e dal De Vis, dal quale traduco le seguenti notizie:

* Il pergolato dell' $A$. macgregoriae si allontana notevolmente da quelli a galleria con volta fatti da altre specie. Intorno ad un arboscello, crescente sul fianco di una ripa, viene fatta una costruzione circolare con un ammasso di ramoscelli e di muschi, ed avente il diametro di 45 pollici, e pareti perpendicolari che si elevano da nove pollici a due piedi; $i$ muschi sono così copiosi da nascondere gli altri materiali. Sulla parte superiore un canale circolare, profondo nove pollici, separa l'arboscello dall'orlo della costruzione; il muro esterno ha nove pollici di spessore ed altrettanti il muro interno, ossia la parte elevata centrale sulla quale sorge I'arboscello. Intorno all'arboscello, fino ad una notevole altezza dalla piattaforma, sono infissi ramoscelli che formano un riparo contro l'avvicinarsi dei nemici dall'alto. La piattaforma superiore è il luogo destinato ai sollazzi degli uccelli; ed ivi si possono vedere parecchi individui dei due sessi inseguirsi girando intorno all'arboscello. Pare che i materiali per le costruzioni siano scelti e trasportati dai maschi alle femmine, le quali soltanto farebbero da architetti ».

(725) Aeluroedus stonei, Sharpe.

Aeluroedus stonei, De Vis, Ann. Rep. Brit. New Guin. p. 62 (1890) (Mount Belford at 4000 feet) - Id., Colon. Papers, no. 103, p. 116 (1890). - Id., Ibis, 1891, p. 40.

Non arendo potuto inserire nelle pagine precedenti tutte le citazioni di un mio recente lavoro intorno alla terza Collezione Ornitologica fatta nella Nuova Guinea Orientale dal Loria, credo opportuno di aggiungerne qui due, che si riferiscono a specie di maggior interesse:

(10) Machaerorhampus alcinus, Westerm.

Machaerorhamphus alcinus, Salvad., Ann. Mus. Civ. Gen. (2) X, p. 799 (Kalo) (1891).

«o ad. Iride giallo-arancio; becco nero; piedi giallo paglia, verdognolo. Si nutre di pesci. Nome volgare Kino »(Loria).

(36) Gircus spilothorax, SALVAD.

Circus spilothorax, Salsad, Ann. Mus. Civ. Gen. (2) X, p. 800 (1891).

Il Loria ha inviato un esemplare, il secondo che si conosca, di questa rara specie; disgraziatamente esso manca (ed è il solo in tutta la collezione) del cartellino, e perciò ignoro la precisa località, nella quale è stato raccolto ; esso è similissimo al tipo, ma ha la tinta nera delle parti superiori e delle macchie longitudinali sulla gola e sul petto meno cupa. 





